\title{
Light-Enabled Radical 1,4-Aryl Migration Via a
}

\section{Phospho-Smiles Rearrangement}

Maxime De Abreu, Philippe Belmont and Etienne Brachet*

Université de Paris, CiTCoM, CNRS, F-75006 Paris, France

etienne.brachet@u-paris.fr

\section{Summary}

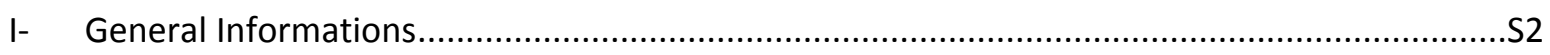

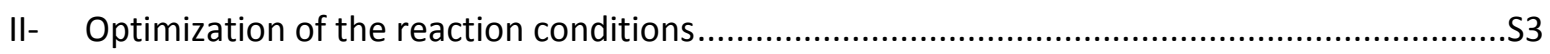

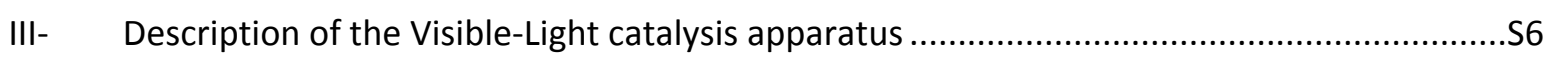

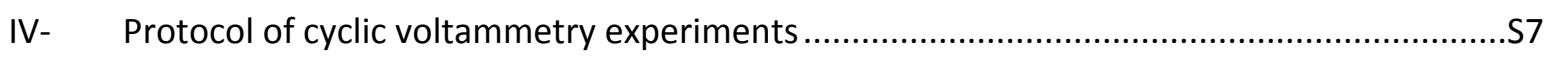

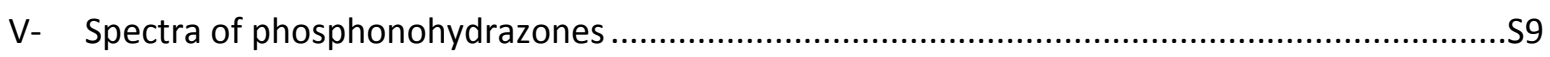

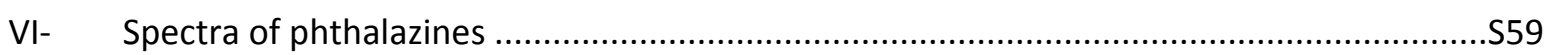

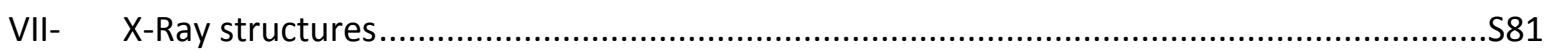

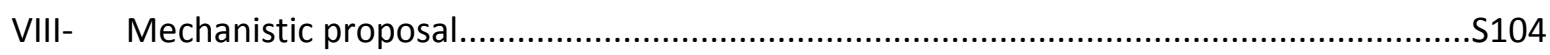

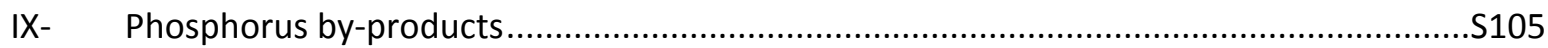

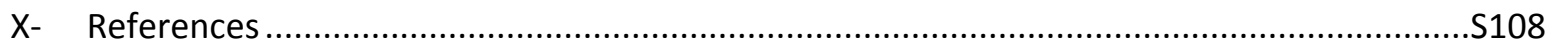




\section{I- $\quad$ General Informations}

${ }^{1} \mathrm{H}$ NMR, ${ }^{13} \mathrm{C}$ NMR, ${ }^{31} \mathrm{P}$ NMR and ${ }^{19} \mathrm{~F}$ NMR spectra were recorded on Bruker Avance 300, 400 or 600 $\mathrm{MHz}$ spectrometers in $\mathrm{CDCl}_{3}$, DMSO- $d_{6}$ or acetone- $d_{6}$ solution with internal solvent signal as reference. NMR data are reported as follows: chemical shift $(\mathrm{ppm})$, multiplicity $(\mathrm{s}=$ singlet, $\mathrm{d}=$ doublet, $\mathrm{t}=$ triplet, $q=$ quartet, quint = quintet,$d d=$ doublet of doublets, $d d d=$ doublet of doublet of doublets, $t d=$ triplet of doublets, $q d=$ quartet of doublets, $m=$ multiplet, br. $\mathrm{s} .=$ broad singlet $)$, and coupling constants $(\mathrm{Hz})$ and number of protons (for ${ }^{1} \mathrm{H}$ NMR). All reactions were monitored by thin-layer chromatography using Merck silica gel plates 60 F254. Visualization was accomplished with short wavelength UV light (254 and $365 \mathrm{~nm}$ ) and/or staining with appropriate stains (anisaldehyde, orthophosphomolybdic acid). Standard flash chromatography was performed using silica gel of particle size $40-63 \mu \mathrm{m}$. Photocatalysts were purchased from Aldrich, $\mathrm{TCl}$, and used without any further purification or synthesized as reported in literature. All other commercially available reagents and solvents were used without further purification. 


\section{II- Optimization of the reaction conditions}

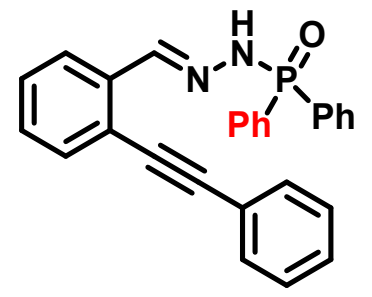

$1 \mathrm{a}$

.

(1)

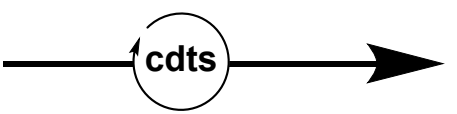

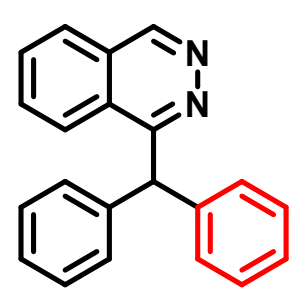

$2 a$

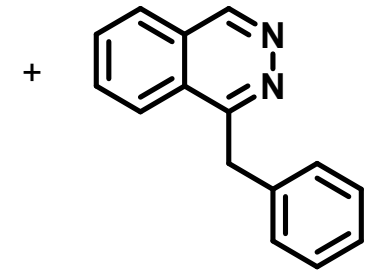

$3 a$

\begin{tabular}{|c|c|c|c|c|c|}
\hline $\mathbf{N}^{\circ}$ & PC \% & Additive & $\begin{array}{c}\text { Solvent } \\
\text { Concentration }\end{array}$ & Yield 2a & Yield 3a \\
\hline & $\mathrm{Ru}(\mathrm{bpy})_{3}\left(\mathrm{PF}_{6}\right)_{2} 2.5 \%$ & tBuONa 3 eq & $\mathrm{MeOH} 0.3 \mathrm{M}$ & $16 \%$ & $15 \%$ \\
\hline & $\mathrm{Ru}(\mathrm{bpy})_{3}\left(\mathrm{PF}_{6}\right)_{2} 2.5 \%$ & tBuONa 3 eq & EtOH $0.3 \mathrm{M}$ & $15 \%$ & $27 \%$ \\
\hline & $\mathrm{Ru}(\mathrm{bpy})_{3} \mathrm{Cl}_{2} .6 \mathrm{H}_{2} \mathrm{O} 2.5 \%$ & tBuONa 3 eq & EtOH $0.3 \mathrm{M}$ & $18 \%$ & $39 \%$ \\
\hline & 4CzIPN 5\% & tBuONa 3 eq & EtOH $0.3 \mathrm{M}$ & I & Traces \\
\hline & Eosin Y 5\% & tBuONa 3 eq & EtOH $0.3 \mathrm{M}$ & l & Traces \\
\hline & Mes Acr+ ClO4- 5\% & tBuONa 3 eq & EtOH $0.3 \mathrm{M}$ & / & Traces \\
\hline & $\mathrm{Ru}(\mathrm{bpy})_{3} \mathrm{Cl}_{2} \cdot 6 \mathrm{H}_{2} \mathrm{O} 2.5 \%$ & $\mathrm{NaOH} 1.5$ eq 3A M.S. & EtOH $0.075 \mathrm{M}$ & $25 \%$ & $64 \%$ \\
\hline & $\mathrm{Ru}(\mathrm{bpy})_{3} \mathrm{Cl}_{2} .6 \mathrm{H}_{2} \mathrm{O} 2.5 \%$ & tBuONa 1.1 eq 3A M.S. & DMSO $0.15 \mathrm{M}$ & / & / \\
\hline & $\mathrm{Ru}(\mathrm{bpy})_{3} \mathrm{Cl}_{2} .6 \mathrm{H}_{2} \mathrm{O} 2.5 \%$ & tBuONa 1.1 eq 3A M.S. & $\mathrm{CHCl} 30.15 \mathrm{M}$ & l & I \\
\hline & $\mathrm{Ru}(\mathrm{bpy})_{3} \mathrm{Cl}_{2} .6 \mathrm{H}_{2} \mathrm{O} 2.5 \%$ & tBuONa 1.1 eq 3A M.S. & Toluene $0.15 \mathrm{M}$ & I & l \\
\hline & $\mathrm{Ru}(\mathrm{bpy})_{3} \mathrm{Cl}_{2} .6 \mathrm{H}_{2} \mathrm{O} 2.5 \%$ & tBuONa 1.1 eq 3A M.S. & MeCN $0.15 \mathrm{M}$ & / & / \\
\hline & $\mathrm{Ru}(\mathrm{bpy})_{3} \mathrm{PF}_{6} 2.5 \%$ & tBuONa 1.1eq 3A M.S. & EtOH $0.3 \mathrm{M}$ & $16 \%$ & $67 \%$ \\
\hline & $\mathrm{Ru}(\mathrm{bpy})_{3} \mathrm{Cl}_{2} .6 \mathrm{H}_{2} \mathrm{O} 2.5 \%$ & Cs2CO3 1.1eq 3A M.S. & EtOH $0.3 \mathrm{M}$ & / & / \\
\hline & $\operatorname{Ir}(\mathrm{ppy})_{3} 2 \%$ & tBuONa 1.1eq & EtOH $0.075 \mathrm{M}$ & I & I \\
\hline & $\left(\operatorname{Ir}\left[\mathrm{dF}\left(\mathrm{CF}_{3}\right) \mathrm{ppy}\right]_{2}(\mathrm{dtbpy})\right) \mathrm{PF}_{6} 2 \%$ & tBuONa 1.1eq & EtOH $0.075 \mathrm{M}$ & $31 \%$ & $27 \%$ \\
\hline & Triphenylpyrilium BF4- 5\% & tBuONa 1.1eq & EtOH $0.15 \mathrm{M}$ & / & I \\
\hline & $\mathrm{Ru}(\mathrm{bpy})_{3} \mathrm{Cl}_{2} .6 \mathrm{H}_{2} \mathrm{O} 2.5 \%$ & $\mathrm{NaOH}$ 1.5eq 3A M.S. & EtOH $0.3 \mathrm{M}$ & $21 \%$ & $39 \%$ \\
\hline & $\mathrm{Ru}(\mathrm{bpy})_{3} \mathrm{Cl}_{2} \cdot 6 \mathrm{H}_{2} \mathrm{O} 2.5 \%$ & $\mathrm{NaOH}$ 3eq 3A M.S. & EtOH $0.075 \mathrm{M}$ & $22 \%$ & $28 \%$ \\
\hline & $\mathrm{Ru}(\mathrm{bpy})_{3} \mathrm{Cl}_{2} .6 \mathrm{H}_{2} \mathrm{O} 2.5 \%$ & $\mathrm{NaOH}$ 3eq 3A M.S. & EtOH $0.3 \mathrm{M}$ & $21 \%$ & $31 \%$ \\
\hline & $\mathrm{Ru}(\mathrm{bpy})_{3} \mathrm{Cl}_{2} .6 \mathrm{H}_{2} \mathrm{O} 2.5 \%$ & K2CO3 1.5 eq 3A M.S. & MeCN $0.075 \mathrm{M}$ & I & I \\
\hline & $\mathrm{Ru}(\mathrm{bpy})_{3} \mathrm{Cl}_{2} .6 \mathrm{H}_{2} \mathrm{O} 2.5 \%$ & $\begin{array}{c}\mathrm{NaOH} 1.5 \text { eq } 3 \mathrm{~A} \text { M.S. } \\
\text { BenzylNEt3+Cl- } \\
25 \mathrm{~mol} \%\end{array}$ & EtOH $0.075 \mathrm{M}$ & $24 \%$ & $30 \%$ \\
\hline & $\mathrm{Ru}(\mathrm{bpy})_{3} \mathrm{Cl}_{2} .6 \mathrm{H}_{2} \mathrm{O} 2.5 \%$ & $\mathrm{NaOH} 1.5$ eq $3 \mathrm{~A}$ M.S. & $\begin{array}{c}\text { Glycerol 98\% } \\
0.075 \mathrm{M}\end{array}$ & / & / \\
\hline & $\mathrm{Ru}(\mathrm{bpy})_{3} \mathrm{Cl}_{2} .6 \mathrm{H}_{2} \mathrm{O} 2.5 \%$ & $\mathrm{NaOH} 1.5$ eq $3 \mathrm{~A}$ M.S. & EtOH $0.01875 \mathrm{M}$ & $45 \%$ & $25 \%$ \\
\hline & $\mathrm{Ru}(\mathrm{bpy})_{3} \mathrm{Cl}_{2} \cdot 6 \mathrm{H}_{2} \mathrm{O} 2.5 \%$ & $\mathrm{NaOH} 1.5$ eq $3 \mathrm{~A}$ M.S. & EtOH $0.0375 \mathrm{M}$ & $35 \%$ & $22 \%$ \\
\hline & $\mathrm{Ru}(\mathrm{bpy})_{3} \mathrm{Cl}_{2} .6 \mathrm{H}_{2} \mathrm{O} 2.5 \%$ & $\mathrm{NaOH} 3$ eq $3 \mathrm{~A}$ M.S. & EtOH $0.01875 \mathrm{M}$ & $36 \%$ & $41 \%$ \\
\hline & $\mathrm{Ru}(\mathrm{bpy})_{3} \mathrm{Cl}_{2} .6 \mathrm{H}_{2} \mathrm{O} 2.5 \%$ & $\mathrm{NaOH} 1.5$ eq $3 \mathrm{~A}$ M.S. & Butanol $0.075 \mathrm{M}$ & $37 \%$ & $44 \%$ \\
\hline & $\mathrm{Ru}(\mathrm{bpy})_{3} \mathrm{Cl}_{2} .6 \mathrm{H}_{2} \mathrm{O} 2.5 \%$ & $\mathrm{NaOH} 1.5$ eq $3 \mathrm{~A}$ M.S. & $\begin{array}{l}\text { Ethanol } 0.01875 \\
\qquad \mathrm{M} 40^{\circ} \mathrm{C}\end{array}$ & $20 \%$ & $57 \%$ \\
\hline & $\mathrm{Ru}(\mathrm{bpy})_{3} \mathrm{Cl}_{2} .6 \mathrm{H}_{2} \mathrm{O} 2.5 \%$ & $\mathrm{NaOH} 1$ eq $3 \mathrm{~A}$ M.S. & $\begin{array}{l}\text { Ethanol } 0.01875 \\
\qquad \mathrm{M} 40^{\circ} \mathrm{C}\end{array}$ & $15 \%$ & $55 \%$ \\
\hline & $\mathrm{Ru}(\mathrm{bpy})_{3} \mathrm{Cl}_{2} .6 \mathrm{H}_{2} \mathrm{O} 2.5 \%$ & $\mathrm{NaOH} 1.5$ eq $3 \mathrm{~A}$ M.S. & $\begin{array}{c}\text { Butanol } 0.01875 \\
\mathrm{M} 40^{\circ} \mathrm{C}\end{array}$ & $38 \%$ & $61 \%$ \\
\hline & $\mathrm{Ru}(\mathrm{bpy})_{3} \mathrm{Cl}_{2} .6 \mathrm{H}_{2} \mathrm{O} 2.5 \%$ & $\mathrm{NaOH} 1.5$ eq $3 \mathrm{~A}$ M.S. & $\mathrm{MeOH} 0.01875 \mathrm{M}$ & $21 \%$ & $28 \%$ \\
\hline
\end{tabular}




\begin{tabular}{|c|c|c|c|c|}
\hline $\mathrm{Ru}(\mathrm{bpy})_{3} \mathrm{Cl}_{2} \cdot 6 \mathrm{H}_{2} \mathrm{O} 2.5 \%$ & $\mathrm{NaOH} 1.5$ eq $3 \mathrm{~A}$ M.S. & $\begin{array}{l}\text { Propan } 1 \mathrm{ol} \\
0.01875 \mathrm{M}\end{array}$ & $36 \%$ & $62 \%$ \\
\hline $\mathrm{Ru}(\mathrm{bpy})_{3} \mathrm{Cl}_{2} .6 \mathrm{H}_{2} \mathrm{O} 2.5 \%$ & NaOH 1.5 eq 3 A M.S. & $\begin{array}{l}\text { Butan } 1 \mathrm{ol} \\
0.01875 \mathrm{M}\end{array}$ & $42 \%$ & $52 \%$ \\
\hline $\mathrm{Ru}(\mathrm{bpy})_{3} \mathrm{Cl}_{2} .6 \mathrm{H}_{2} \mathrm{O} 2.5 \%$ & $\mathrm{NaOH} 1.5$ eq $3 \mathrm{~A}$ M.S. & DME $0.01875 \mathrm{M}$ & I & I \\
\hline $\mathrm{Ru}(\mathrm{bpy})_{3} \mathrm{Cl}_{2} .6 \mathrm{H}_{2} \mathrm{O} 2.5 \%$ & NaOH 1.5 eq 3 A M.S. & $\begin{array}{c}\text { EtOH/DMSO } \\
6 \mathrm{~mL} / 2 \mathrm{~mL} 0.01875 \\
\mathrm{M}\end{array}$ & $47 \%$ & $38 \%$ \\
\hline $\mathrm{Ru}(\mathrm{bpy})_{3} \mathrm{Cl}_{2} \cdot 6 \mathrm{H}_{2} \mathrm{O} 2.5 \%$ & $\mathrm{NaOH} 1.5$ eq $3 \mathrm{~A}$ M.S. & $\begin{array}{c}\text { EtOH/DMSO } \\
7.5 \mathrm{~mL} / 0.5 \mathrm{~mL} \\
0.01875 \mathrm{M}\end{array}$ & $67 \%$ & $28 \%$ \\
\hline $\mathrm{Ru}(\mathrm{bpy})_{3} \mathrm{Cl}_{2} \cdot 6 \mathrm{H}_{2} \mathrm{O} 2.5 \%$ & $\mathrm{NaOH} 1.5$ eq $3 \mathrm{~A}$ M.S. & $\begin{array}{c}\text { EtOH/DMSO } \\
7.5 \mathrm{~mL} / 0.5 \mathrm{~mL} \\
0.01875 \mathrm{M}\end{array}$ & $48 \%$ & Traces \\
\hline $\mathrm{Ru}(\mathrm{bpy})_{3} \mathrm{Cl}_{2} .6 \mathrm{H}_{2} \mathrm{O} 2.5 \%$ & $\mathrm{NaOH} 1.5$ eq $3 \mathrm{~A}$ M.S. & $\begin{array}{c}\text { EtOH/DMSO } \\
4 \mathrm{~mL} / 4 \mathrm{~mL} 0.01875 \\
M\end{array}$ & $40 \%$ & $1 \%$ \\
\hline $\mathrm{Ru}(\mathrm{bpy})_{3} \mathrm{Cl}_{2} .6 \mathrm{H}_{2} \mathrm{O} 2.5 \%$ & NaOH 1.5 eq 3 A M.S. & $\begin{array}{c}\text { EtOH/DMSO } \\
2 \mathrm{~mL} / 6 \mathrm{~mL} 0.01875 \\
\mathrm{M}\end{array}$ & $14 \%$ & $20 \%$ \\
\hline $\mathrm{Ru}(\mathrm{bpy})_{3} \mathrm{Cl}_{2} .6 \mathrm{H}_{2} \mathrm{O} 2.5 \%$ & $\mathrm{NaOH} 1.5$ eq $3 \mathrm{~A}$ M.S. & HFIP 0.01875 M & I & I \\
\hline $\mathrm{Ru}(\mathrm{bpy})_{3} \mathrm{Cl}_{2} \cdot 6 \mathrm{H}_{2} \mathrm{O} 2.5 \%$ & $\mathrm{NaOH} 1.5$ eq $3 \mathrm{~A}$ M.S. & $\begin{array}{c}\text { EtOH/Acétone } \\
4 \mathrm{~mL} / 4 \mathrm{~mL} 0.01875 \\
\mathrm{M}\end{array}$ & $35 \%$ & Traces \\
\hline $\mathrm{Ru}(\mathrm{bpy})_{3} \mathrm{Cl}_{2} .6 \mathrm{H}_{2} \mathrm{O} 2.5 \%$ & $\mathrm{NaOH} 1.5$ eq $3 \mathrm{~A}$ M.S. & $\begin{array}{c}\text { EtOH/Acétone } \\
7.5 \mathrm{~mL} / 0.5 \mathrm{~mL} \\
0.01875 \mathrm{M}\end{array}$ & $37 \%$ & Traces \\
\hline $\mathrm{Ru}(\mathrm{bpy})_{3} \mathrm{Cl}_{2} \cdot 6 \mathrm{H}_{2} \mathrm{O} 2.5 \%$ & $\mathrm{NaOH} 1.5$ eq $3 \mathrm{~A}$ M.S. & $\begin{array}{c}\text { EtOH/DMSO } \\
7.5 \mathrm{~mL} / 0.5 \mathrm{~mL} \\
0.01875 \mathrm{M}\end{array}$ & $46 \%$ & Traces \\
\hline $\mathrm{Ru}(\mathrm{bpy})_{3} \mathrm{Cl}_{2} .6 \mathrm{H}_{2} \mathrm{O} 2.5 \%$ & $\mathrm{NaOH} 1.5$ eq $3 \mathrm{~A}$ M.S. & $\begin{array}{c}\text { EtOH/Acétone } \\
7.5 \mathrm{~mL} / 0.5 \mathrm{~mL} \\
0.01875 \mathrm{M}\end{array}$ & $38 \%$ & Traces \\
\hline $\mathrm{Ru}(\mathrm{bpy})_{3} \mathrm{Cl}_{2} .6 \mathrm{H}_{2} \mathrm{O} 2.5 \%$ & $\mathrm{NaOH} 1.5$ eq $3 \mathrm{~A}$ M.S. & $\begin{array}{c}\mathrm{EtOH} / \mathrm{MeCN} \\
7.5 \mathrm{~mL} / 0.5 \mathrm{~mL} \\
0.01875 \mathrm{M}\end{array}$ & $58 \%$ & Traces \\
\hline $\mathrm{Ru}(\mathrm{bpy})_{3} \mathrm{Cl}_{2} .6 \mathrm{H}_{2} \mathrm{O} 2.5 \%$ & $\mathrm{NaOH} 1.5$ eq $3 \mathrm{~A}$ M.S. & $\begin{array}{c}\mathrm{EtOH} / \mathrm{MeCN} \\
2 \mathrm{~mL} / 6 \mathrm{~mL} 0.01875 \\
\mathrm{M}\end{array}$ & $62 \%$ & Traces \\
\hline $\mathrm{Ru}(\mathrm{bpy})_{3} \mathrm{Cl}_{2} .6 \mathrm{H}_{2} \mathrm{O} 2.5 \%$ & $\mathrm{NaOH} 1.5$ eq $3 \mathrm{~A}$ M.S. & $\begin{array}{c}\mathrm{EtOH} / \mathrm{MeCN} \\
6 \mathrm{~mL} / 2 \mathrm{~mL} 0.01875 \\
\mathrm{M}\end{array}$ & $65 \%$ & Traces \\
\hline $\mathrm{Ru}(\mathrm{bpy})_{3} \mathrm{Cl}_{2} \cdot 6 \mathrm{H}_{2} \mathrm{O} 2.5 \%$ & $\mathrm{NaOH} 1.5$ eq $3 \mathrm{~A}$ M.S. & $\begin{array}{c}\mathrm{EtOH} / \mathrm{MeCN} \\
6 \mathrm{~mL} / 2 \mathrm{~mL} 0.0375 \\
M\end{array}$ & $53 \%$ & $25 \%$ \\
\hline Ru(bpy) ${ }_{3} @ S i O 2$ 8mg/g 2.5 \% & $\mathrm{NaOH} 1.5$ eq $3 \mathrm{~A}$ M.S. & $\begin{array}{c}\mathrm{EtOH} / \mathrm{MeCN} \\
6 \mathrm{~mL} / 2 \mathrm{~mL} 0.01875 \\
M\end{array}$ & l & / \\
\hline $\mathrm{Ru}(\mathrm{bpy})_{3} \mathrm{Cl}_{2} .6 \mathrm{H}_{2} \mathrm{O} 2.5 \%$ & $\mathrm{NaOH} 1.5$ eq $3 \mathrm{~A}$ M.S. & $\begin{array}{c}\mathrm{EtOH} / \mathrm{MeCN} \\
12 \mathrm{~mL} / 4 \mathrm{~mL} \\
0.009375 \mathrm{M}\end{array}$ & $53 \%$ & Traces \\
\hline
\end{tabular}




\begin{tabular}{|c|c|c|c|c|}
\hline $\mathrm{Ru}(\text { phen })_{3}\left(\mathrm{PF}_{6}\right)_{2} 2.5 \%$ & $\mathrm{NaOH} 1.5$ eq $3 \mathrm{~A}$ M.S. & $\begin{array}{c}\mathrm{EtOH} / \mathrm{MeCN} \\
6 \mathrm{~mL} / 2 \mathrm{~mL} 0.01875 \\
\mathrm{M}\end{array}$ & $28 \%$ & $28 \%$ \\
\hline $\mathrm{Ru}(\mathrm{bpz})_{3}\left(\mathrm{PF}_{6}\right)_{2} 2.5 \%$ & $\mathrm{NaOH} 1.5$ eq $3 \mathrm{~A}$ M.S. & $\begin{array}{c}\mathrm{EtOH} / \mathrm{MeCN} \\
6 \mathrm{~mL} / 2 \mathrm{~mL} 0.01875 \\
\mathrm{M}\end{array}$ & / & Traces \\
\hline $\mathrm{Ru}(\mathrm{bpy})_{3} \mathrm{Cl}_{2} \cdot 6 \mathrm{H}_{2} \mathrm{O} 1 \%$ & NaOH 1.5 eq 3 A M.S. & $\begin{array}{c}\mathrm{EtOH} / \mathrm{MeCN} \\
6 \mathrm{~mL} / 2 \mathrm{~mL} 0.01875 \\
\mathrm{M}\end{array}$ & $53 \%$ & Traces \\
\hline $\mathrm{Ru}(\mathrm{bpy})_{3} \mathrm{Cl}_{2} \cdot 6 \mathrm{H}_{2} \mathrm{O} 5 \%$ & $\mathrm{NaOH} 1.5$ eq $3 \mathrm{~A}$ M.S. & $\begin{array}{c}\mathrm{EtOH} / \mathrm{MeCN} \\
6 \mathrm{~mL} / 2 \mathrm{~mL} 0.01875 \\
\mathrm{M}\end{array}$ & $69 \%$ & Traces \\
\hline Eosin Y 5\% & $\mathrm{NaOH} 1.5$ eq $3 \mathrm{~A}$ M.S. & $\begin{array}{c}\mathrm{EtOH} / \mathrm{MeCN} \\
6 \mathrm{~mL} / 2 \mathrm{~mL} 0.01875 \\
M\end{array}$ & / & / \\
\hline Rose Bengale 5\% & $\mathrm{NaOH} 1.5$ eq $3 \mathrm{~A}$ M.S. & $\begin{array}{c}\mathrm{EtOH} / \mathrm{MeCN} \\
6 \mathrm{~mL} / 2 \mathrm{~mL} 0.01875 \\
\mathrm{M}\end{array}$ & / & / \\
\hline $\begin{array}{c}\mathrm{Ru}(\mathrm{bpy})_{3} \mathrm{Cl}_{2} \cdot 6 \mathrm{H}_{2} \mathrm{O} 2.5 \% \\
\text { No light }\end{array}$ & $\mathrm{NaOH} 1.5$ eq $3 \mathrm{~A}$ M.S. & $\begin{array}{c}\mathrm{EtOH} / \mathrm{MeCN} \\
6 \mathrm{~mL} / 2 \mathrm{~mL} 0.01875 \\
\mathrm{M}\end{array}$ & / & / \\
\hline $\mathrm{Ru}(\mathrm{bpy})_{3} \mathrm{Cl}_{2} .6 \mathrm{H}_{2} \mathrm{O} 2.5 \%$ & / & $\begin{array}{c}\mathrm{EtOH} / \mathrm{MeCN} \\
6 \mathrm{~mL} / 2 \mathrm{~mL} 0.01875 \\
M\end{array}$ & / & / \\
\hline / & $\mathrm{NaOH} 1.5$ eq $3 \mathrm{~A}$ M.S. & $\begin{array}{c}\mathrm{EtOH} / \mathrm{MeCN} \\
6 \mathrm{~mL} / 2 \mathrm{~mL} 0.01875 \\
\mathrm{M}\end{array}$ & / & / \\
\hline $\begin{array}{c}\mathrm{Ru}(\mathrm{bpy})_{3} \mathrm{Cl}_{2} \cdot 6 \mathrm{H}_{2} \mathrm{O} 2.5 \% \\
\text { SUNLIGHT }\end{array}$ & $\mathrm{NaOH} 1.5$ eq $3 \mathrm{~A}$ M.S. & $\begin{array}{c}\mathrm{EtOH} / \mathrm{MeCN} \\
6 \mathrm{~mL} / 2 \mathrm{~mL} 0.01875 \\
\mathrm{M}\end{array}$ & $38 \%$ & Traces \\
\hline
\end{tabular}

Table S1: Optimization of the reaction conditions 


\section{III- Description of the Visible-Light catalysis apparatus}

\section{1-Apparatus used during optimization and scope investigations}

Our Visible-Light catalysis apparatus consists in an aluminium block in which circulates the cooling fluid linked to the cooling system set at $20^{\circ} \mathrm{C}$. The block contains 6 holes and each hole contain a $18 \mathrm{~W}$ $455 \mathrm{~nm}$ LED Cree ${ }^{\circledR}$ XLamp $^{\circledR}$ XT-E Royal Blue LEDs $(\lambda=455 \mathrm{~nm}( \pm 5 \mathrm{~nm}), 12 \mathrm{~V}, 1.5$ A. The sample are irradiated with a LED through the vial's plane bottom side at a distance of $1 \mathrm{~cm}$. The vials used are WICOM $20 \mathrm{~mm}$ Crimp Top Vial, $5 \mathrm{ml}, 38.5 \mathrm{~mm} \times 22.0 \mathrm{~mm}$, clear. This apparatus is installed on a magnetic stirrer, as shown on the following scheme and picture.

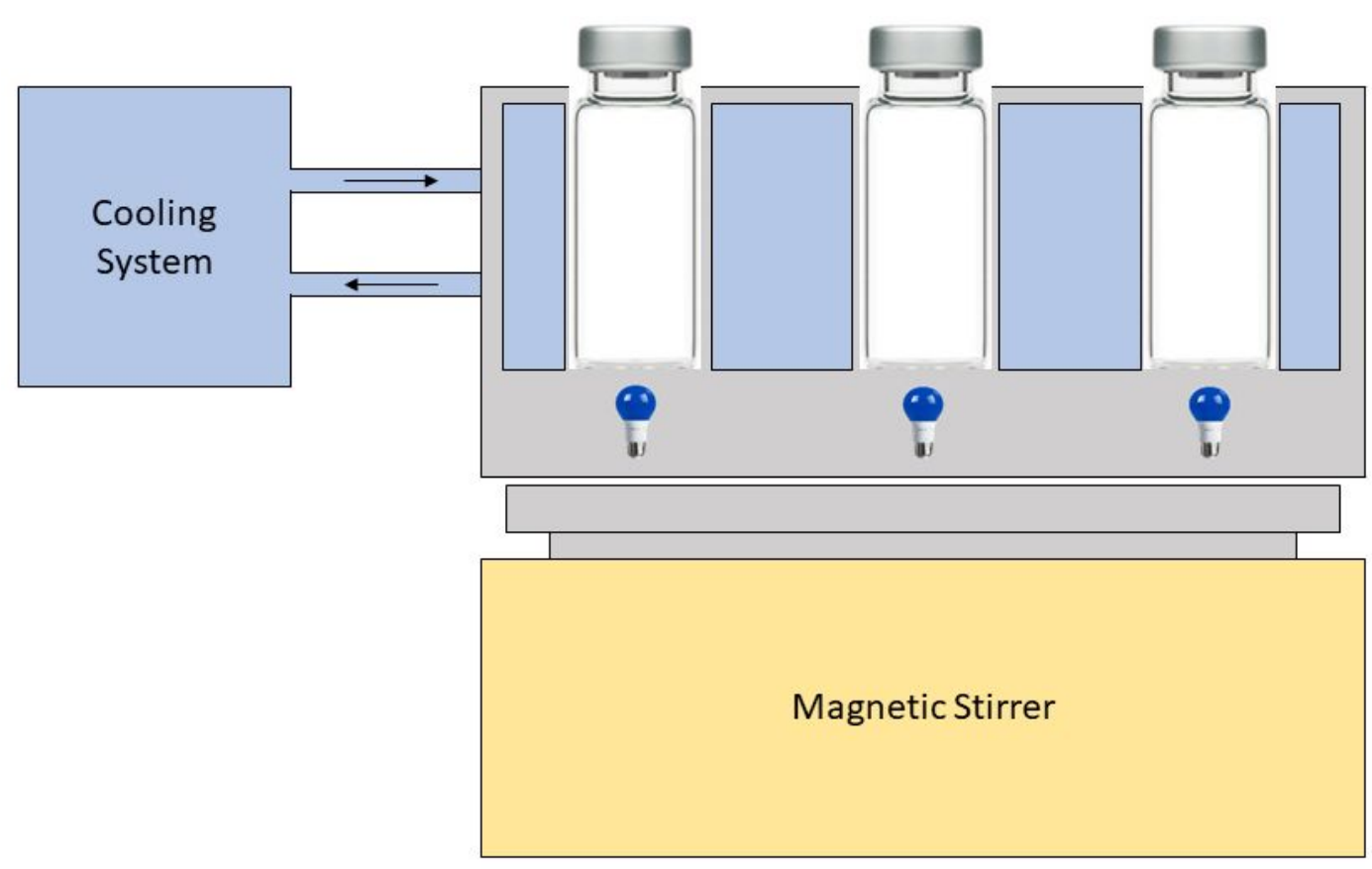

Figure S1 - Sketch of the Visible Light catalysis apparatus 


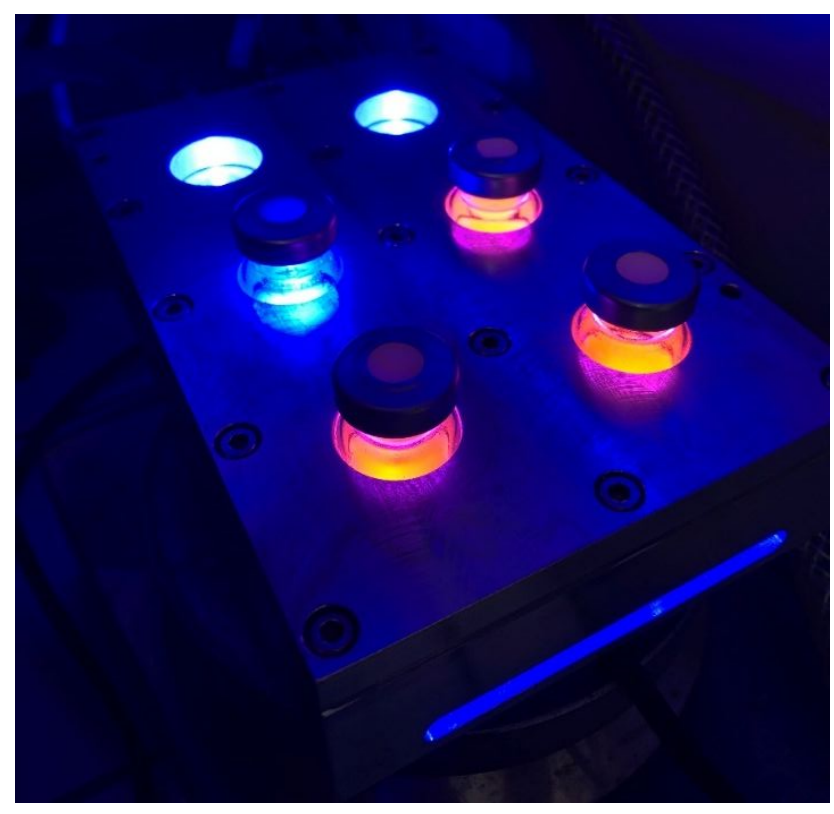

Figure S2 - Picture of the Visible Light catalysis apparatus

2-gram-scale experiment

To

an ove

dried

balloon

was

added

P,P-diphenyl-N'-(2(phenylethynyl)benzylidene)phosphinic hydra-zide (2.378 mmol, 1 eq., $1 \mathrm{~g})$, sodium hydroxide (3.56 mmol, 1.5 eq.), tris(2,2'-bipyridyl)dichlororuthenium(II) hexahydrate $(0.059 \mathrm{mmol}, 2.5$ $\mathrm{mol} \%$ ) and a mixture of $127 \mathrm{ml}$ of $3 / 1 \mathrm{EtOH} / \mathrm{MeCN}$. The balloon was stirred under visible-light irradiation 18W blue LED lamp (CREE XPE, $34 \mathrm{~mW} / \mathrm{cm}^{2}$, ref : HCK1012-01-002, sell by hepatochem : https://www.hepatochem.com/photoreactors-leds-accessories-old/ledevoluchem/) placed at ca. $4 \mathrm{~cm}$ of the flask (see provided pictures). Temperature was maintained by a fan (see picture). In that case however, only traces of the desired product were observed along with an important degradation of the starting material. These observations could be attributed to different light source used in this experiment. 


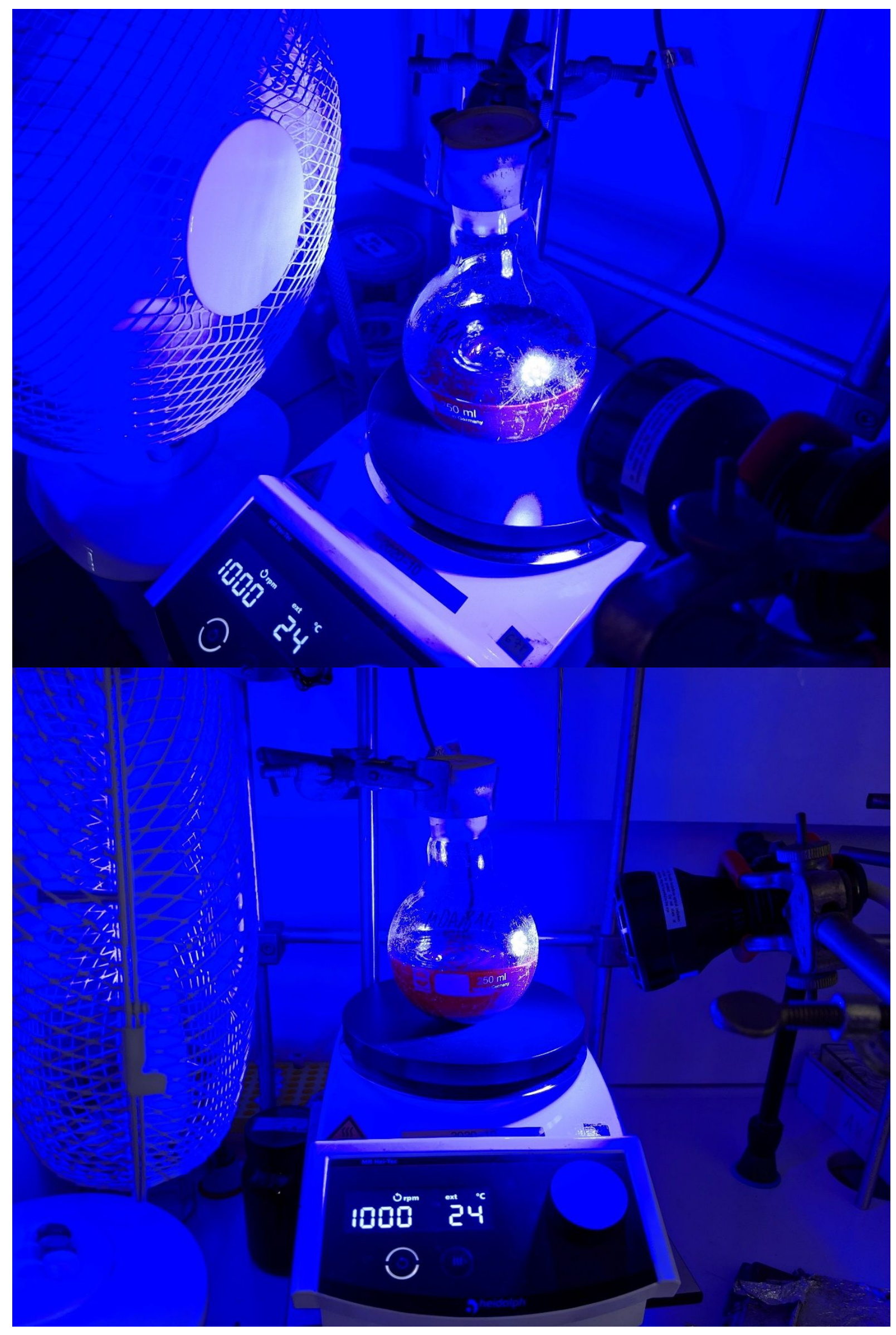




\section{IV- Protocol of cyclic voltammetry experiments}

The redox potentials have been measured by cyclic voltammetry using a glassy-carbon electrode in a $10^{-3} \mathrm{M}$ solution of phosphonohydrazones (1 eq.) with 1.5 eq. of sodium hydroxide, in degassed mixture of ethanol and acetonitrile $(3: 1)$ under argon atmosphere. Scan rate: $0.1 \mathrm{~V} / \mathrm{s}$, Saturated Calomel Electrode (SCE) used as reference electrode, platinum wire as counter electrode and LiClO4 $(0.05 \mathrm{M})$ as the supporting electrolyte. The cyclic voltammogram were treated using Nova 1.6 software. In the presence of 1.5 eq. of base, it is worth noting that the oxidation potential corresponds to the anionic form of the starting material.
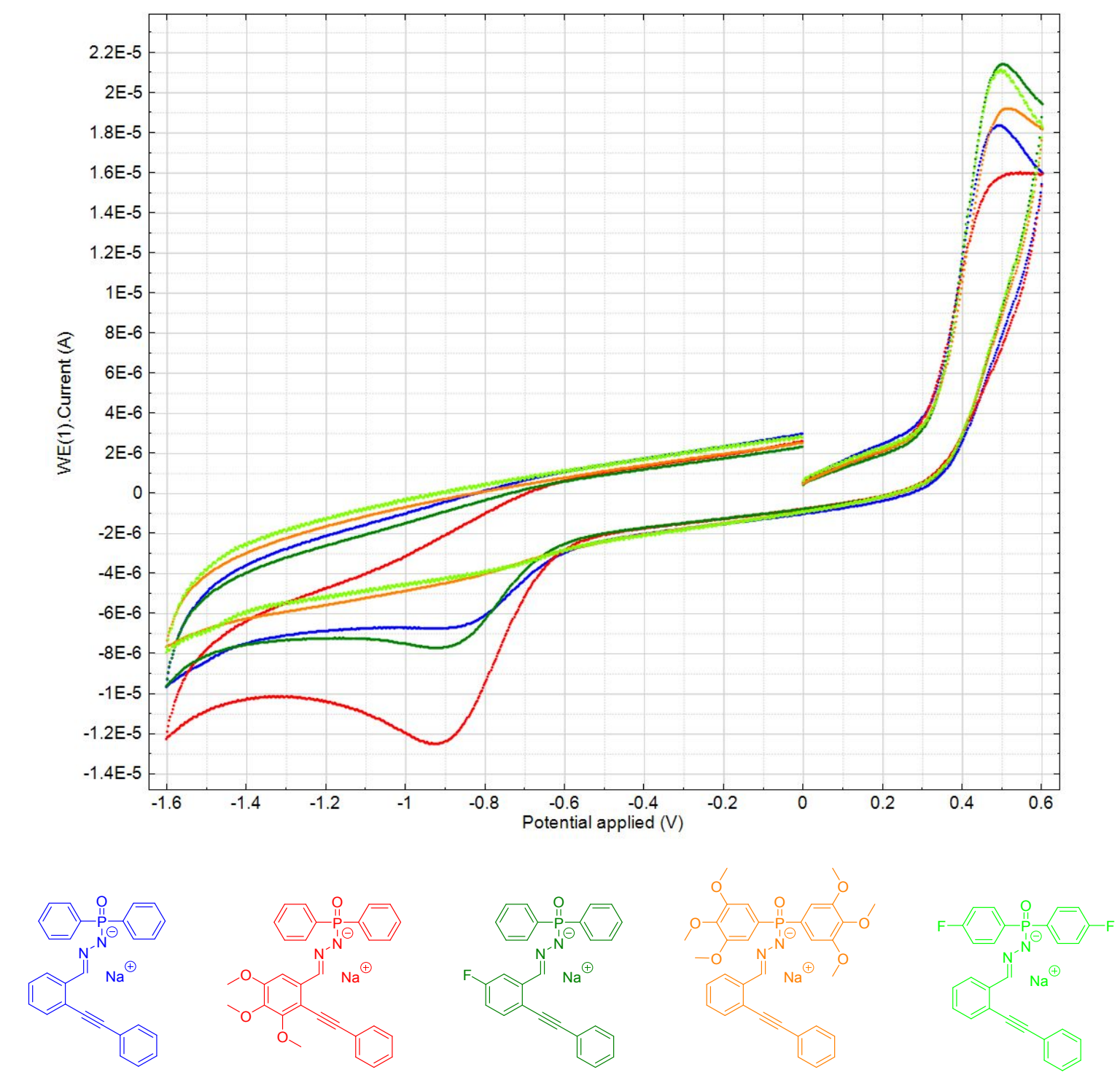

$\mathrm{E}_{\mathrm{Ox}}=+0,5 \mathrm{~V}$ vs. SCE

Figure S3 - Cyclic voltammetry of select compounds in the presence of base

The redox potentials have also been measured in the absence of $\mathrm{NaOH}$ (base), using a glassy carbon electrode in a $10^{-3} \mathrm{M}$ solution of phosphonohydrazones (1 eq.), in degassed mixture of ethanol and acetonitrile (3:1) under argon atmosphere. Scan rate: $0.1 \mathrm{~V} / \mathrm{s}$, Saturated Calomel Electrode (SCE) used as reference electrode, platinum wire as counter electrode and $\mathrm{LiClO}_{4}(0.05 \mathrm{M})$ as the 
supporting electrolyte. Without base, no oxidation potentials are observed and thus indicates that the base is crucial to generate the $\mathrm{N}$-anion intermediate.
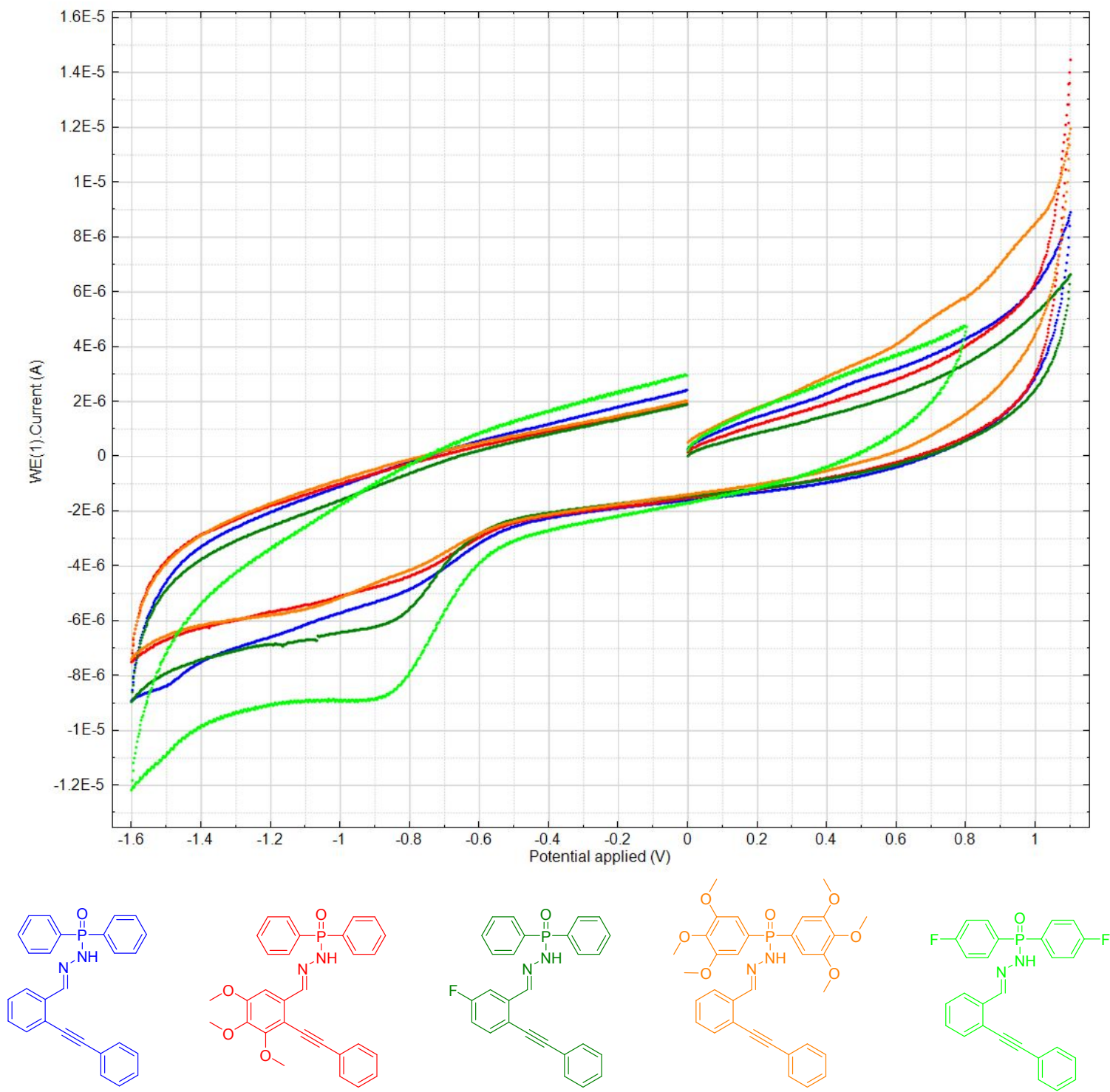

Figure S4 - Cyclic voltammetry of select compounds in the absence of base 
V- $\quad$ Spectra of phosphonohydrazones

Compound 1b: P,P-diphenyl-N'-(2-(p-tolylethynyl)benzylidene)phosphinic hydrazide ${ }^{1}$ H NMR (400 MHz, Chloroform-d)

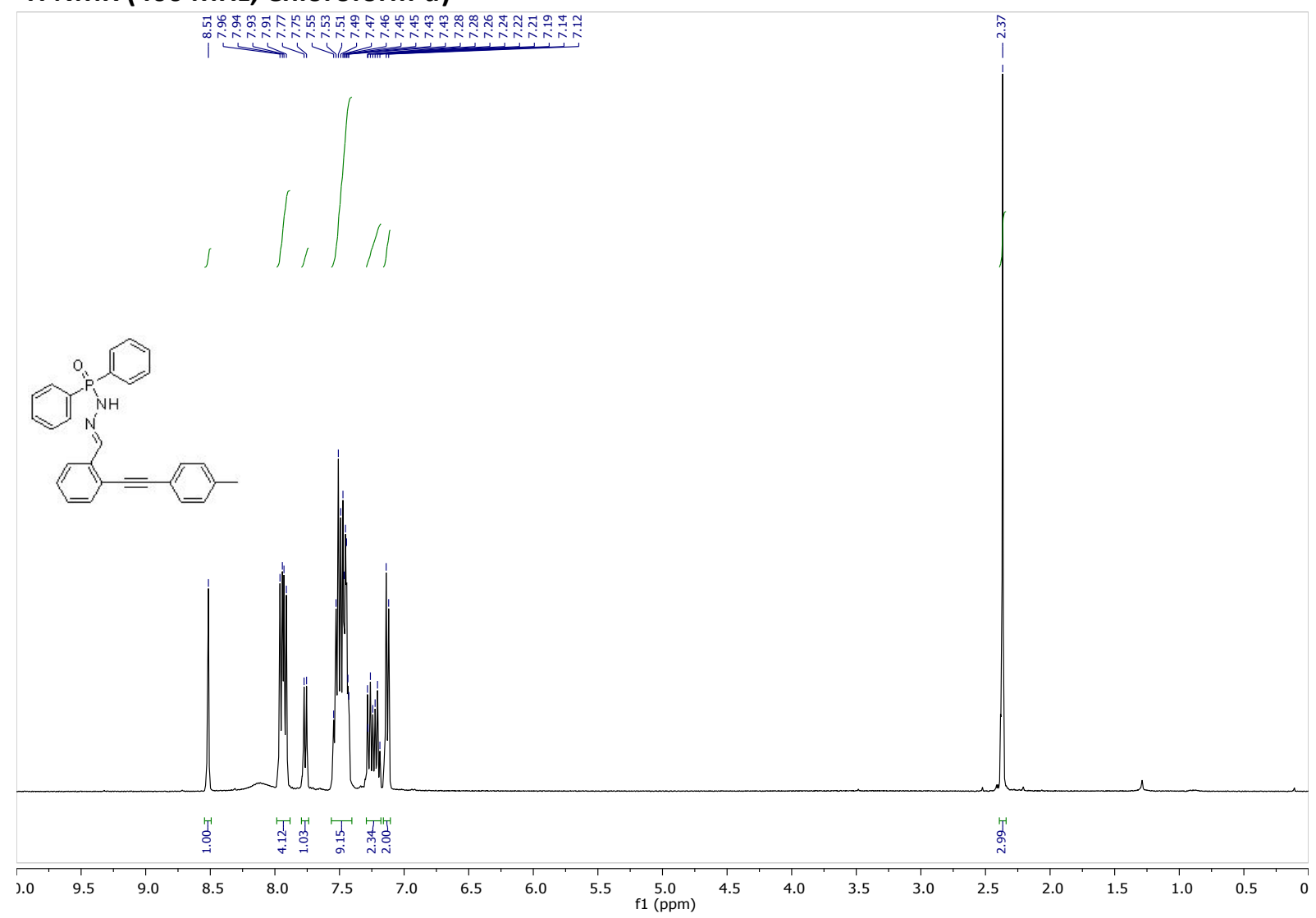

${ }^{13} \mathrm{C}\left\{{ }^{1} \mathrm{H}\right\}$ NMR (101 MHz, Chloroform-d) (JMOD) spectrum 


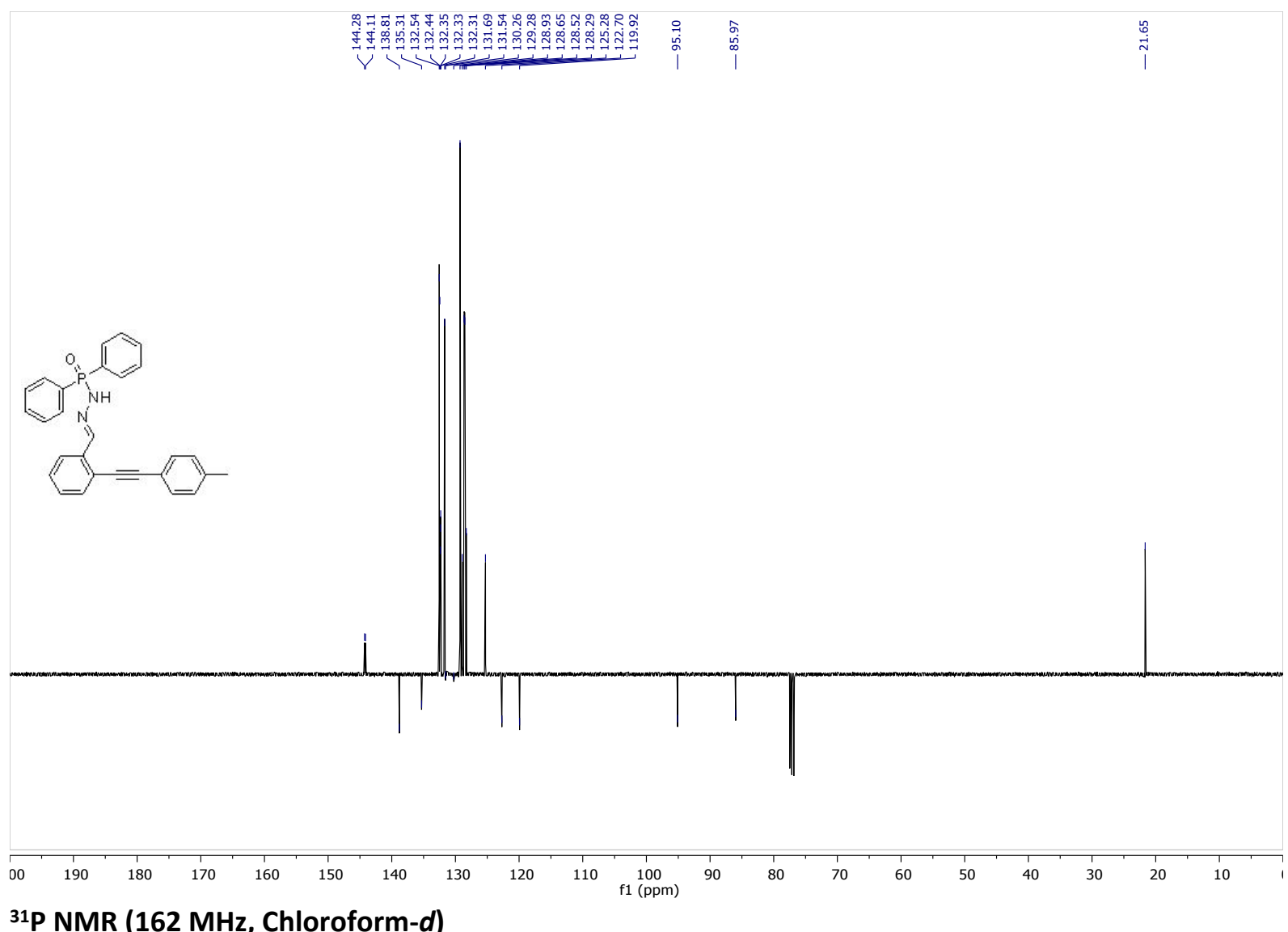

31P NMR (162 MHz, Chloroform-d)
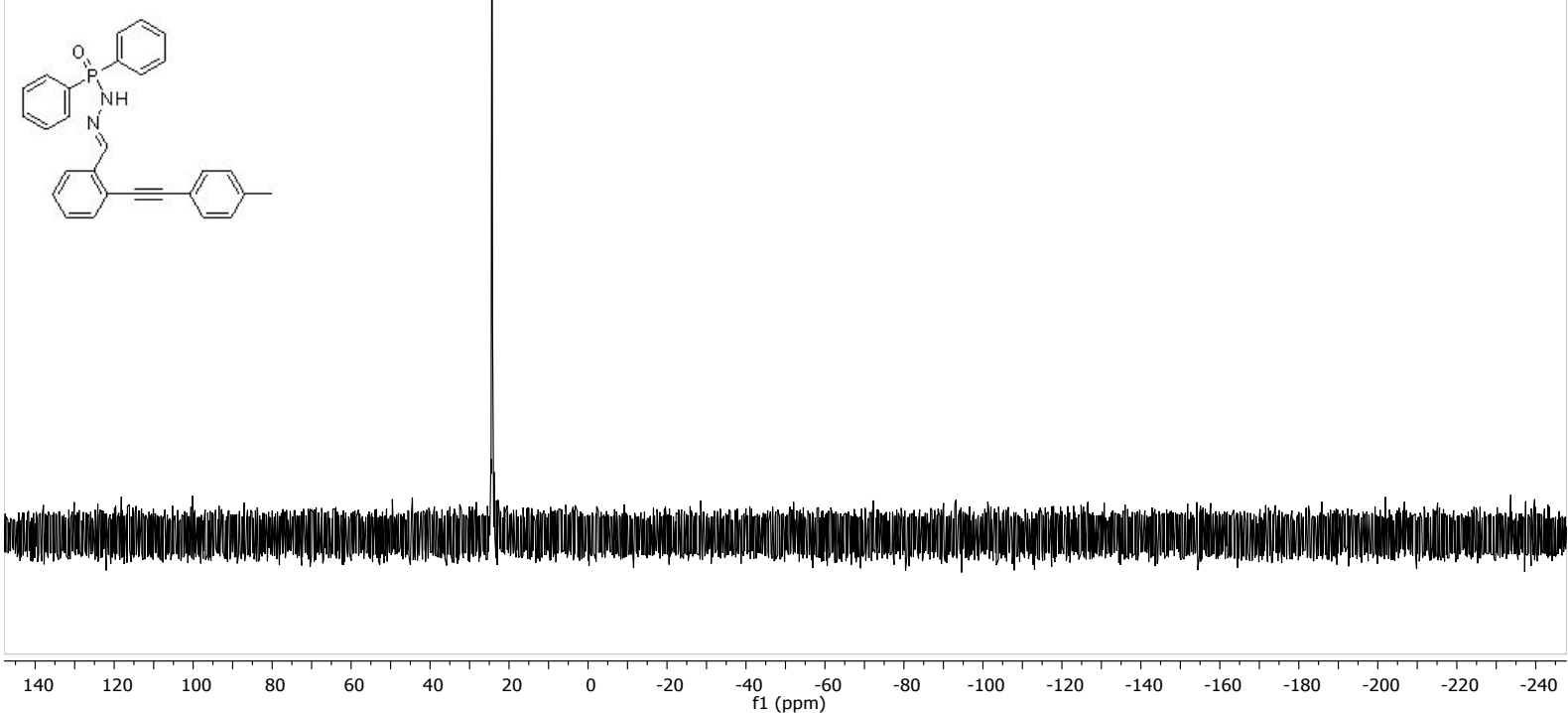

S13 
Compound 1c: N'-(2-((4-fluorophenyl)ethynyl)benzylidene)-P,P-diphenylphosphinic hydrazide ${ }^{1} \mathrm{H}$ NMR (400 MHz, Chloroform-d)

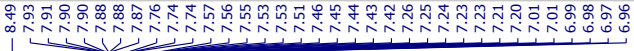
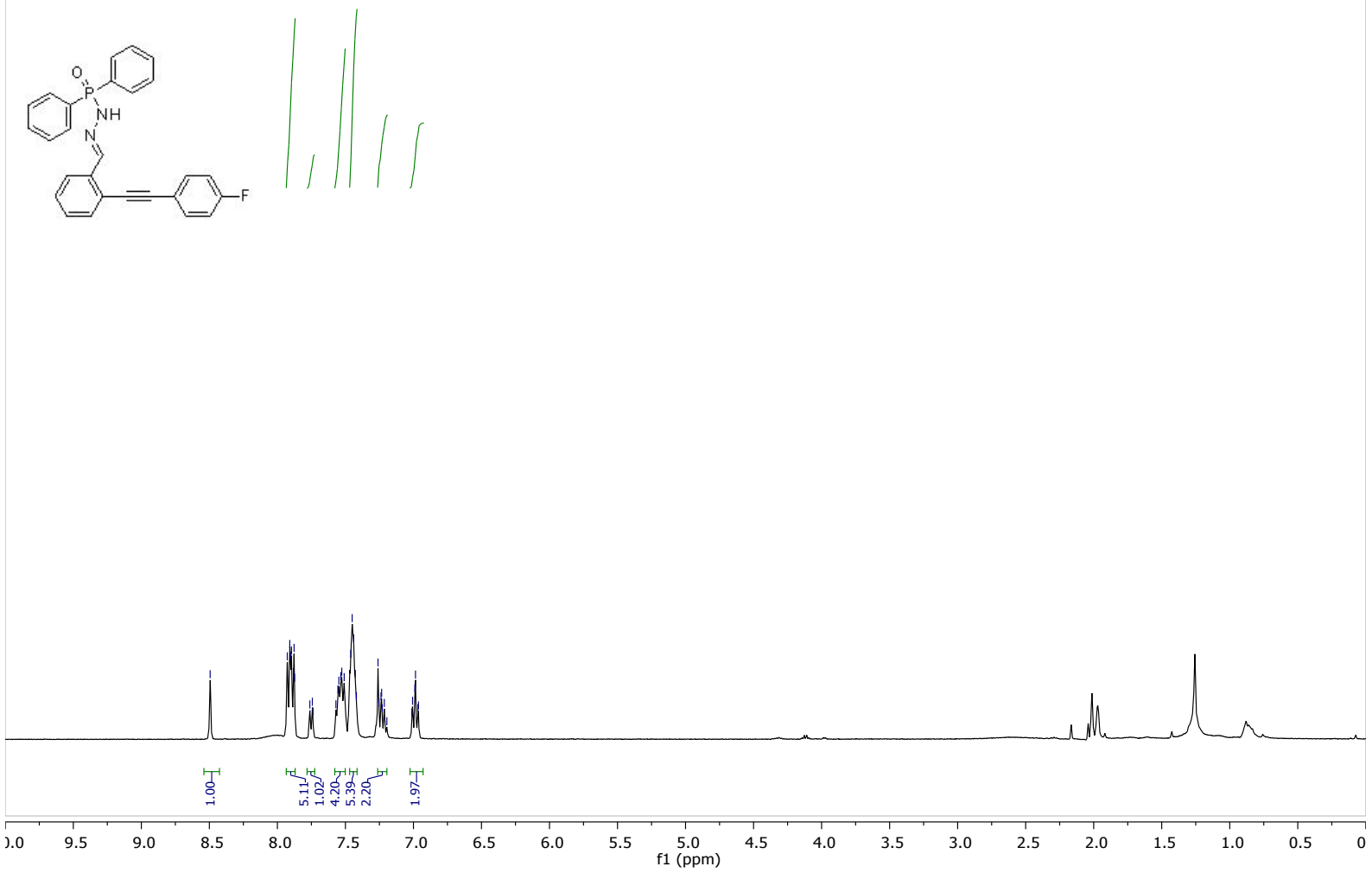

${ }^{13} \mathrm{C}\left\{{ }^{1} \mathrm{H}\right\}$ NMR (101 MHz, Chloroform-d) (JMOD) spectrum

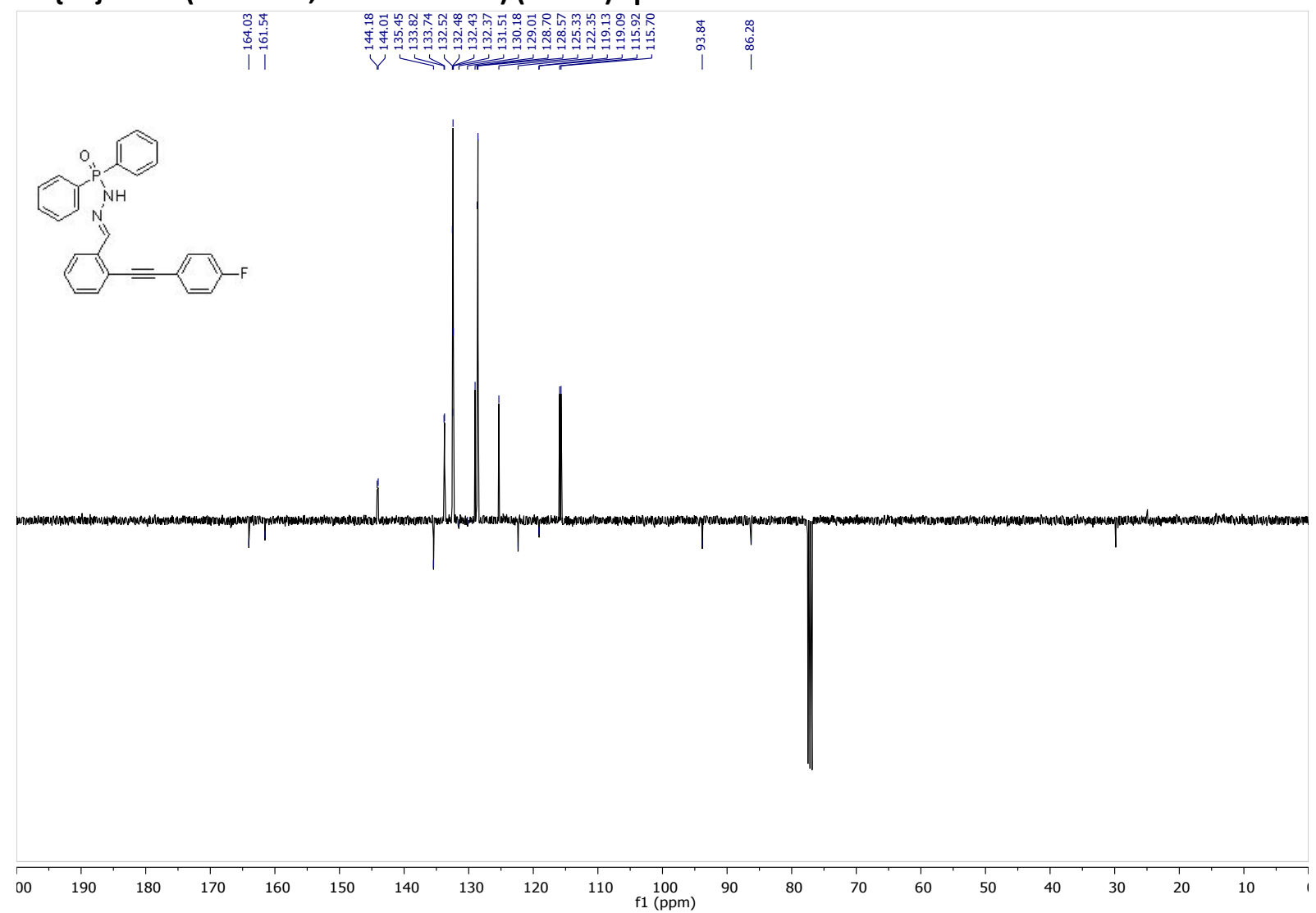


${ }^{31} \mathrm{P}$ NMR (162 MHz, Chloroform-d)
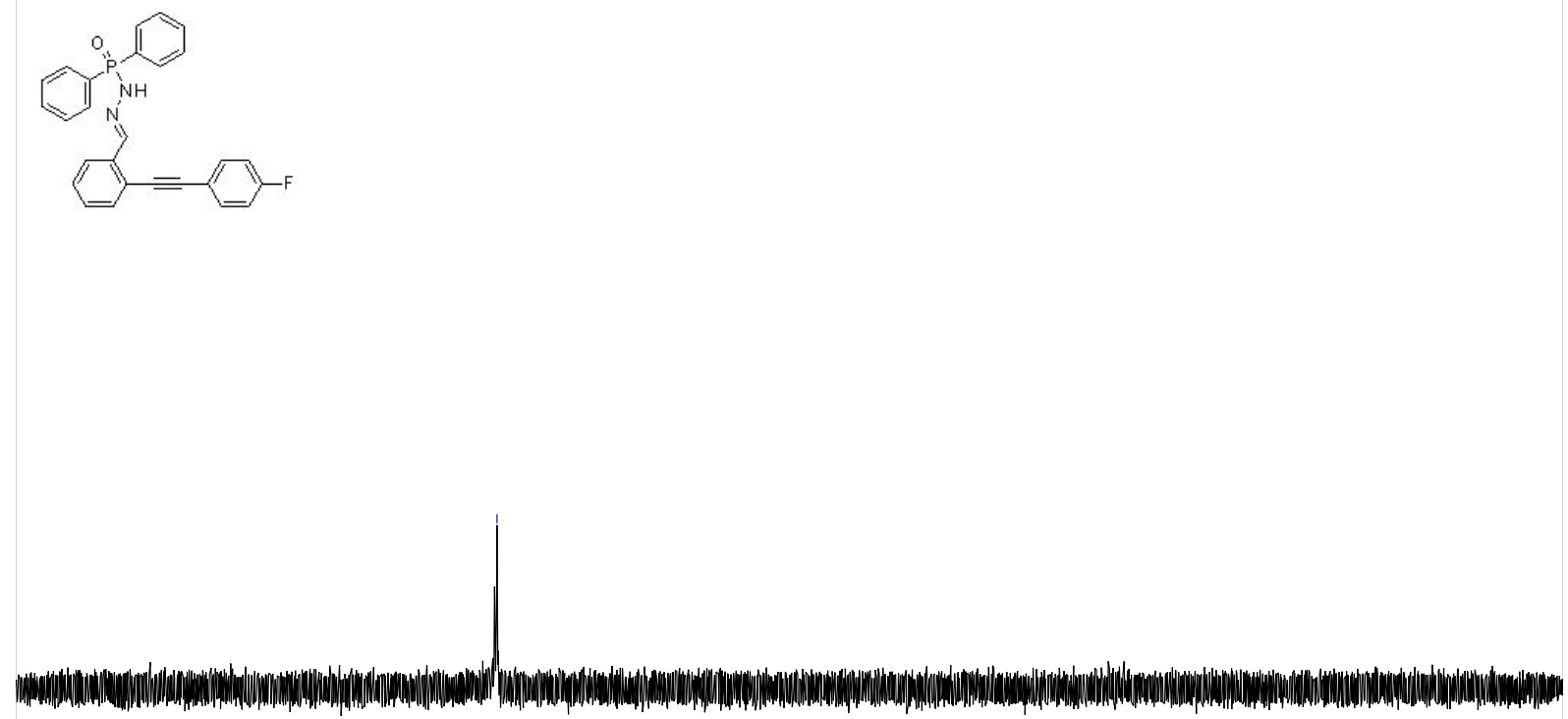

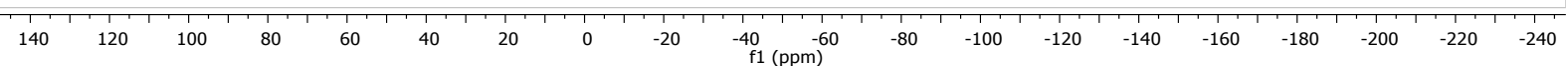

${ }^{19}$ F NMR (376 MHz, Chloroform-d)
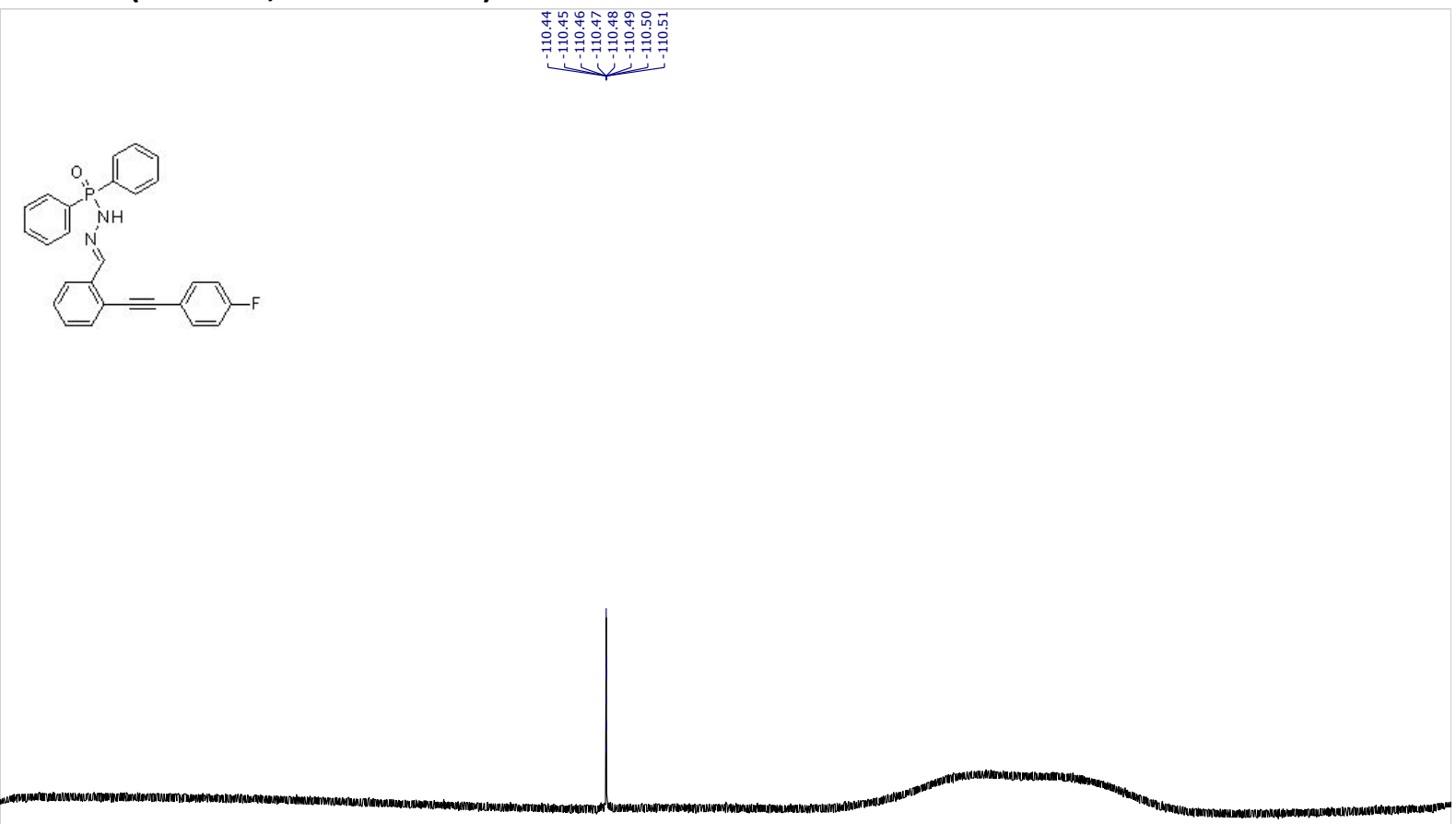

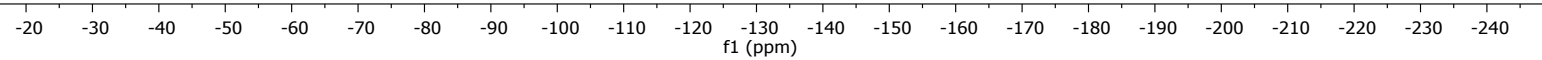


Compound 1d: N'-(2-((6-methoxynaphthalen-2-yl)ethynyl)benzylidene)-P,P-diphenylphosphinic hydrazide

${ }^{1} \mathrm{H}$ NMR (300 MHz, Chloroform-d)

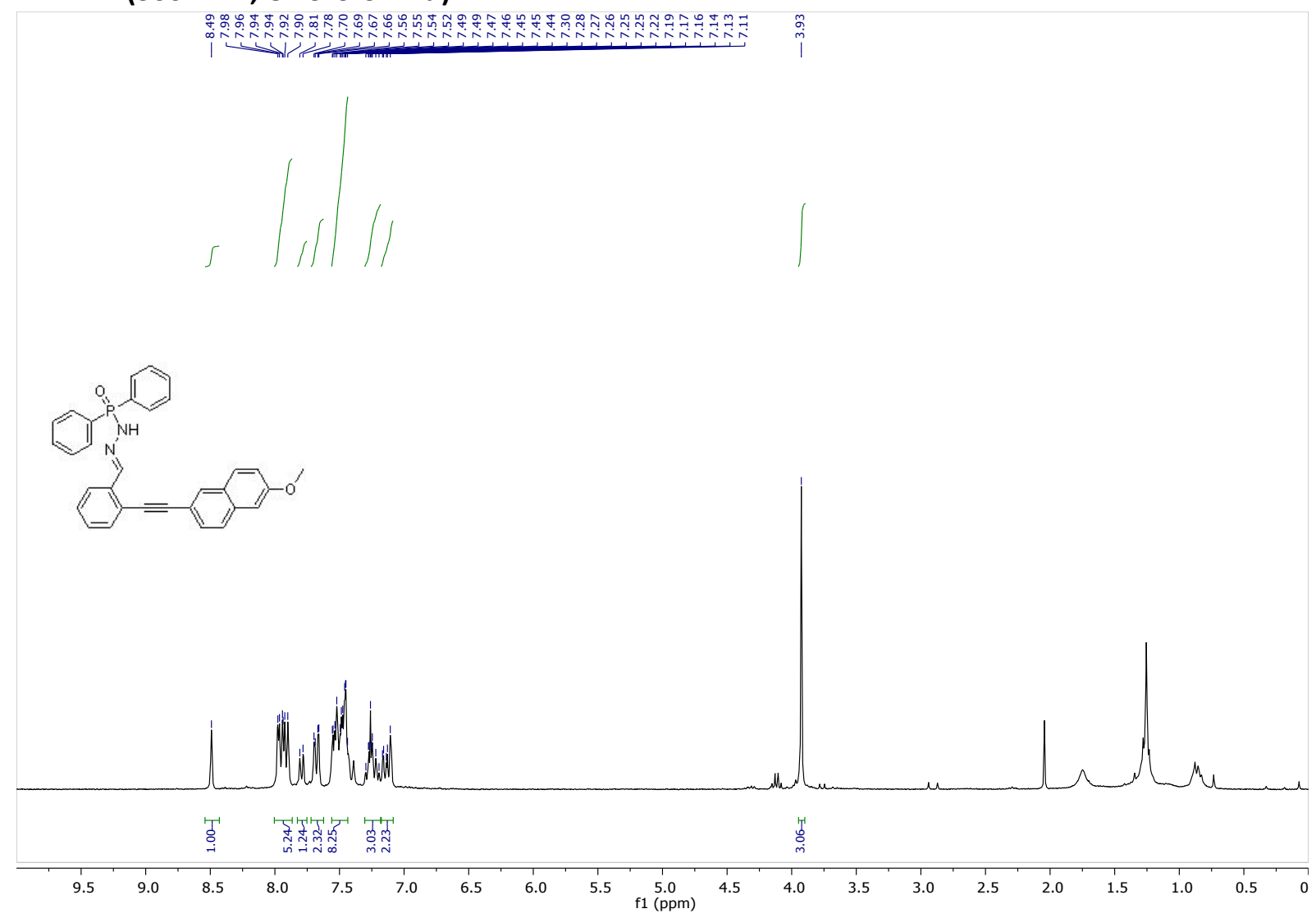

${ }^{13} \mathrm{C}\left\{{ }^{1} \mathrm{H}\right\}$ NMR (75 MHz, Chloroform-d)

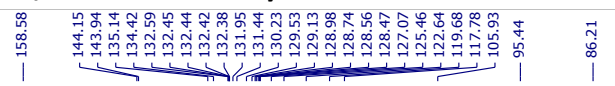
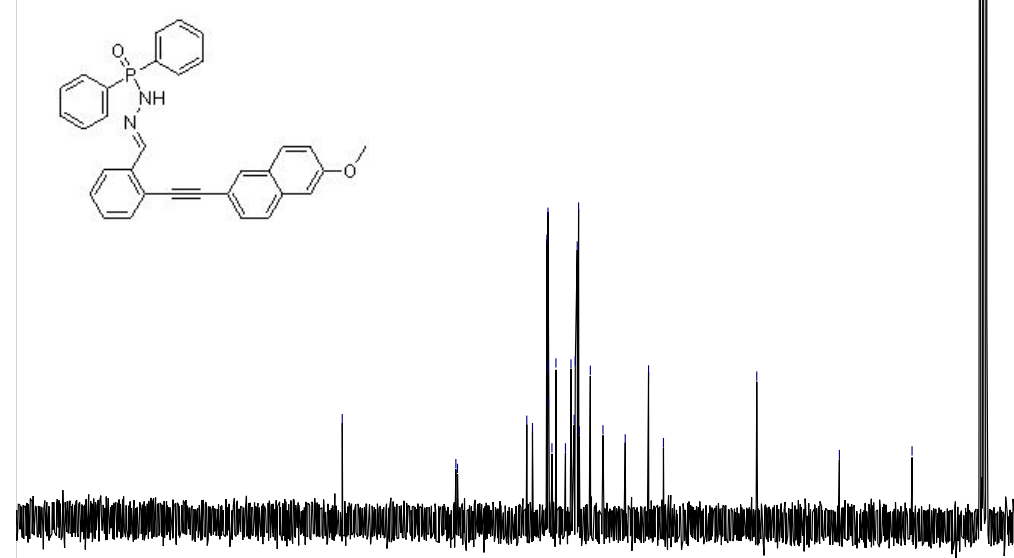

DO

$\begin{array}{llllllllll}190 & 180 & 170 & 160 & 150 & 140 & 130 & 120 & 110 & \begin{array}{c}100 \\ \mathrm{f} 1(\mathrm{ppm})\end{array}\end{array}$

$90 \quad 80$

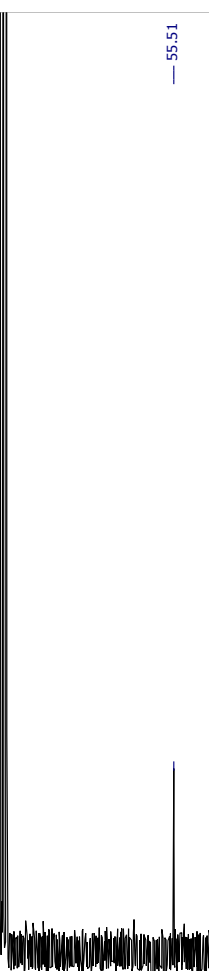


31P NMR (121 MHz, Chloroform-d)

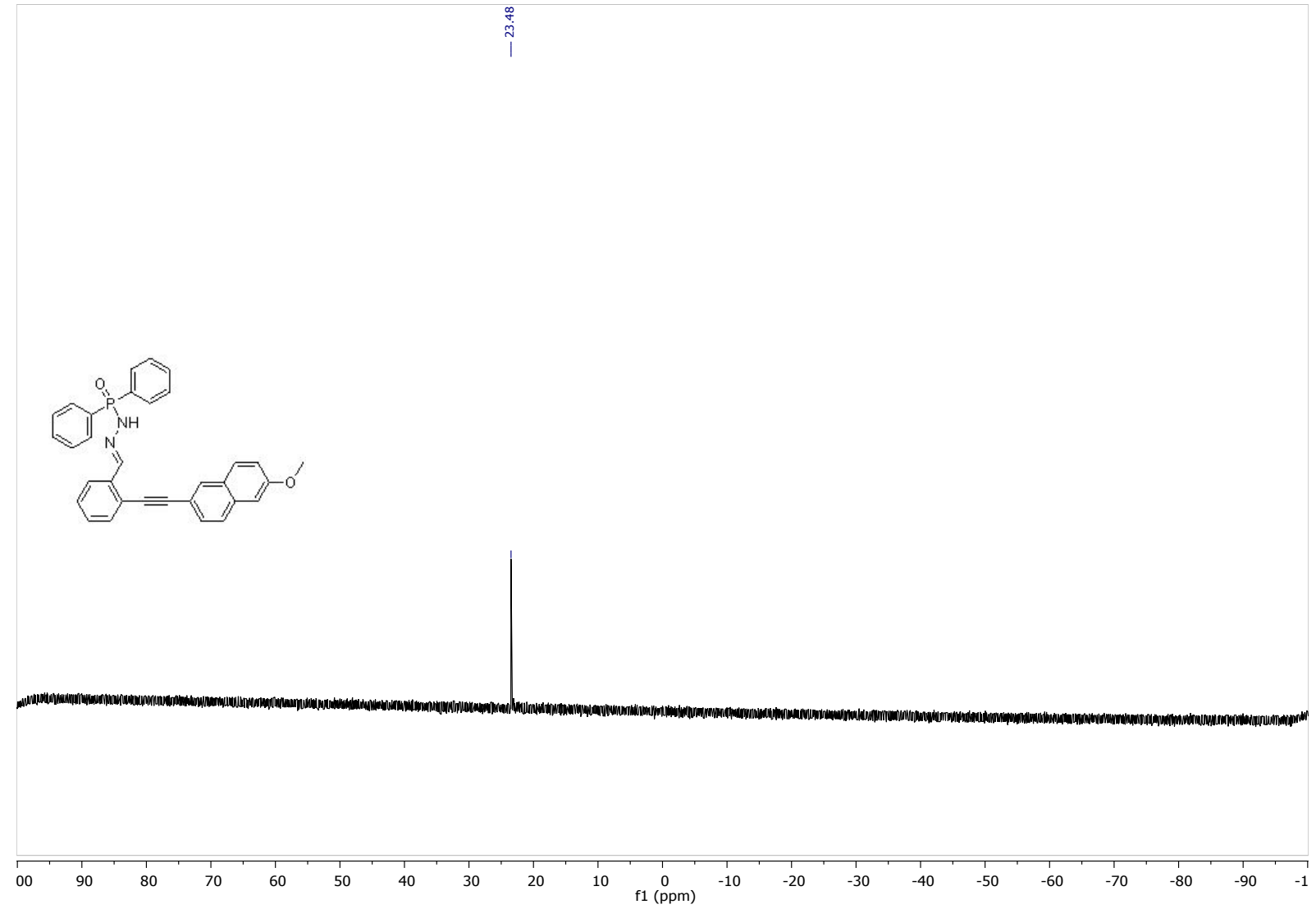


Compound 1e: $\mathrm{N}^{\prime}$-(2-(dibenzo[b,d]thiophen-2-ylethynyl)benzylidene)-P,P-diphenylphosphinic hydrazide

${ }^{1} \mathrm{H}$ NMR (300 MHz, Chloroform-d)

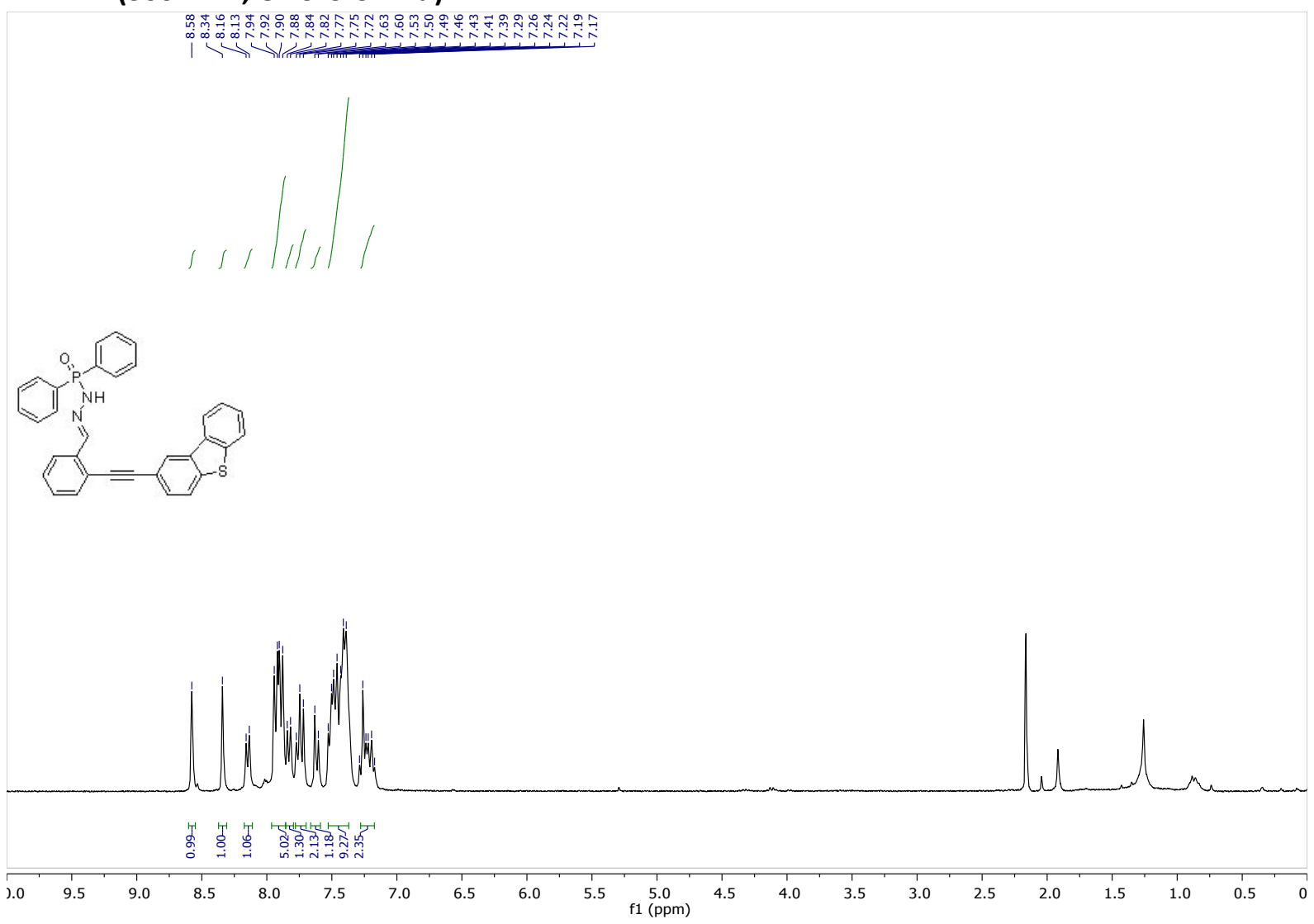

${ }^{13} \mathrm{C}\left\{{ }^{1} \mathrm{H}\right\}$ NMR (75 MHz, Chloroform-d)

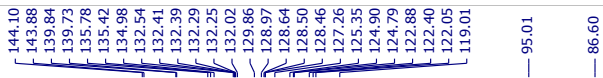

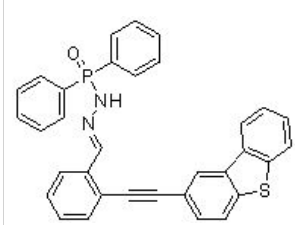

00

190

$180 \quad 170 \quad 160$

$\begin{array}{llllll}150 & 140 & 130 & 120 & 110 & \begin{array}{c}100 \\ \mathrm{f} 1(\mathrm{ppm})\end{array}\end{array}$

80

60

50

40 
31P NMR (121 MHz, Chloroform-d)

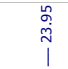

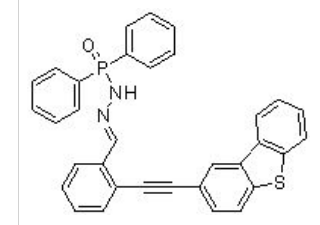

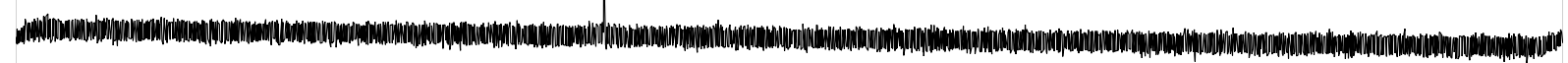

\begin{tabular}{lllllllllllllllllllll}
\hline 0 & 90 & 80 & 70 & 60 & 50 & 40 & 30 & 20 & 10 & $\underset{\mathrm{f} 1}{\mathrm{f}(\mathrm{ppm})}$ & -10 & -20 & -30 & -40 & -50 & -60 & -70 & -80 & -90 & -1
\end{tabular}


Compound 1f: N'-(2-(pent-1-yn-1-yl)benzylidene)-P,P-diphenylphosphinic hydrazide

${ }_{1}^{1}$ H NMR (400 MHz, Chloroform-d)
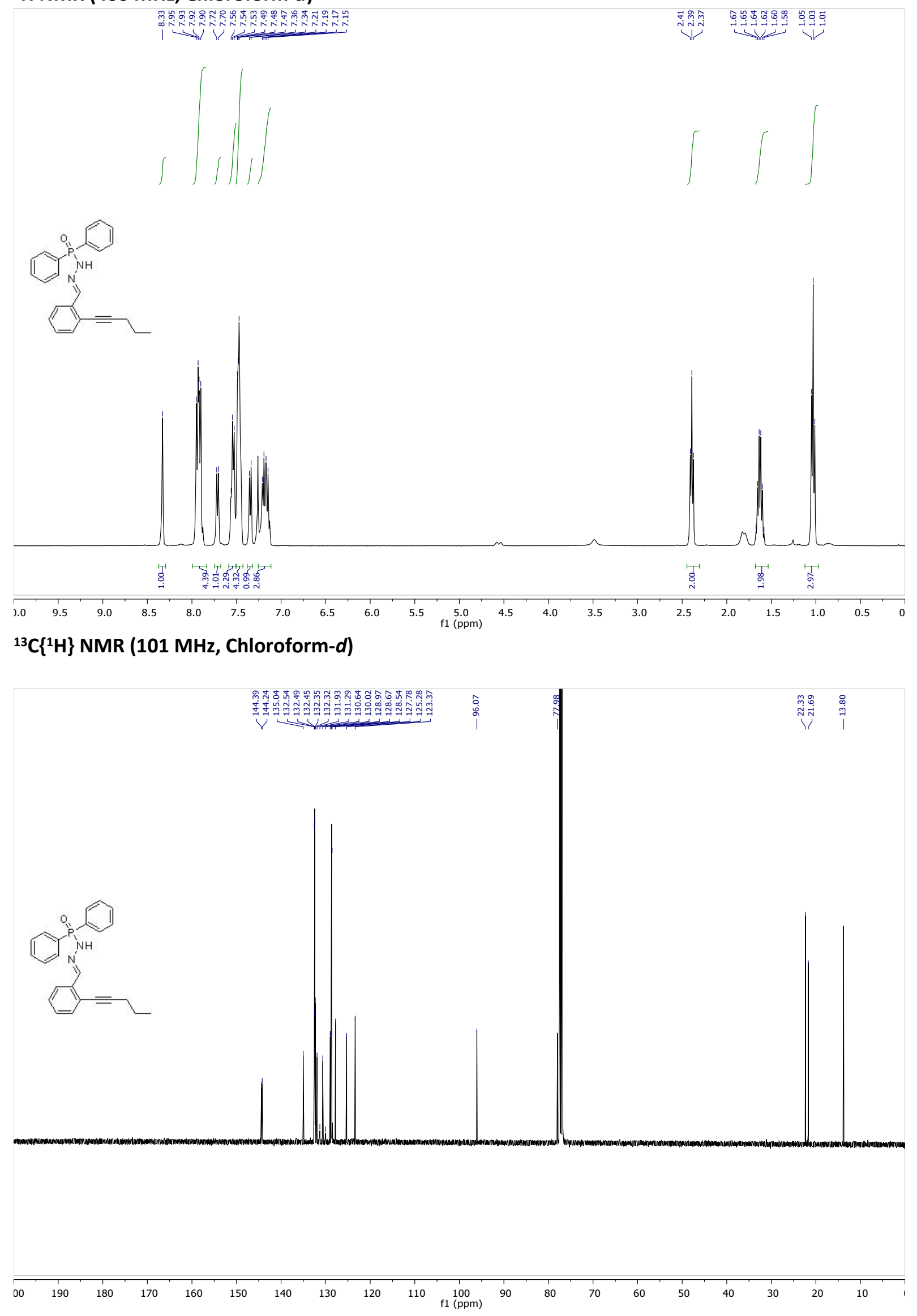

S20 
31P NMR (162 MHz, Chloroform-d)

亭
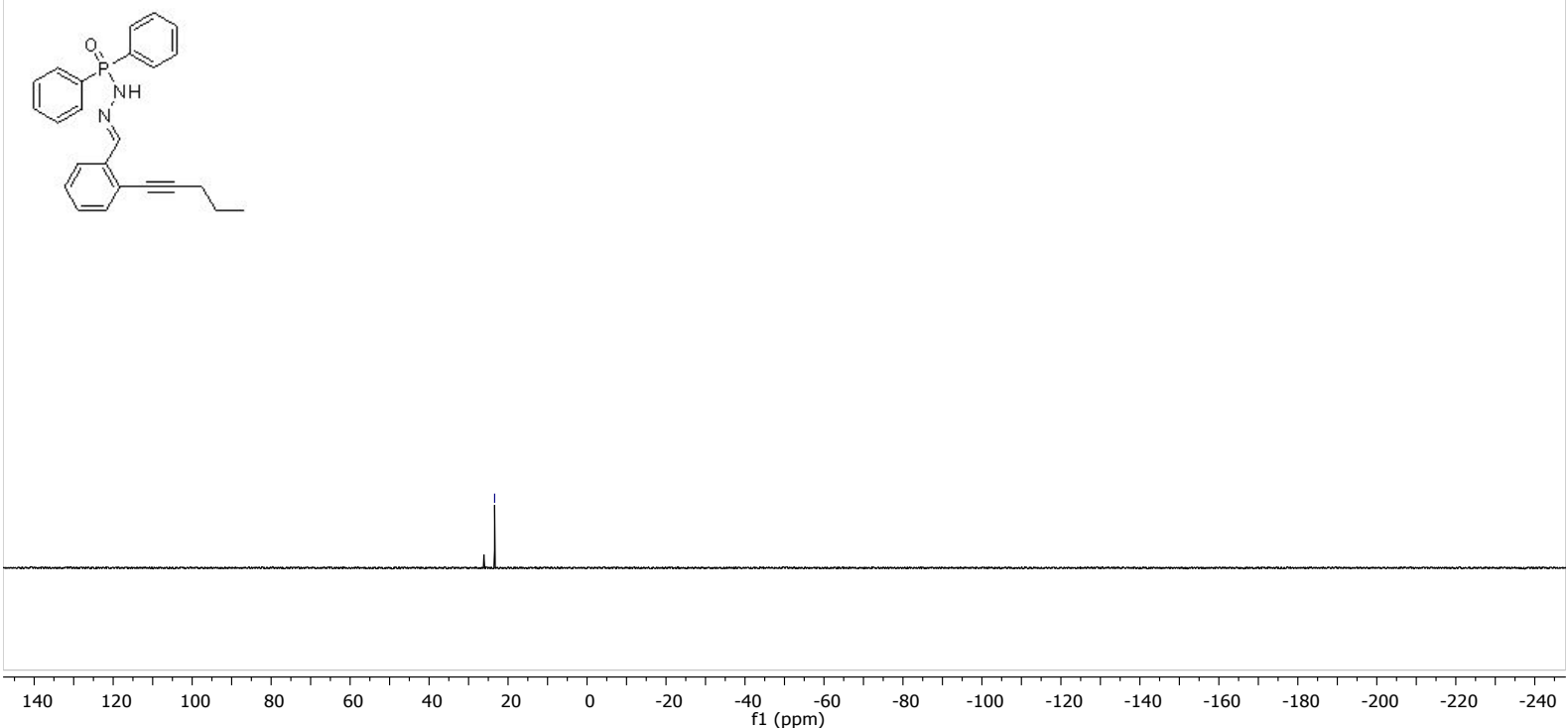
Compound 1g: N'-(2-(3,3-dimethylbut-1-yn-1-yl)benzylidene)-P,P-diphenylphosphinic hydrazide ${ }^{1} \mathrm{H}$ NMR (300 MHz, Chloroform-d)

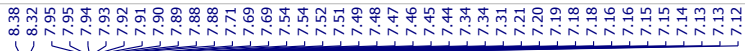

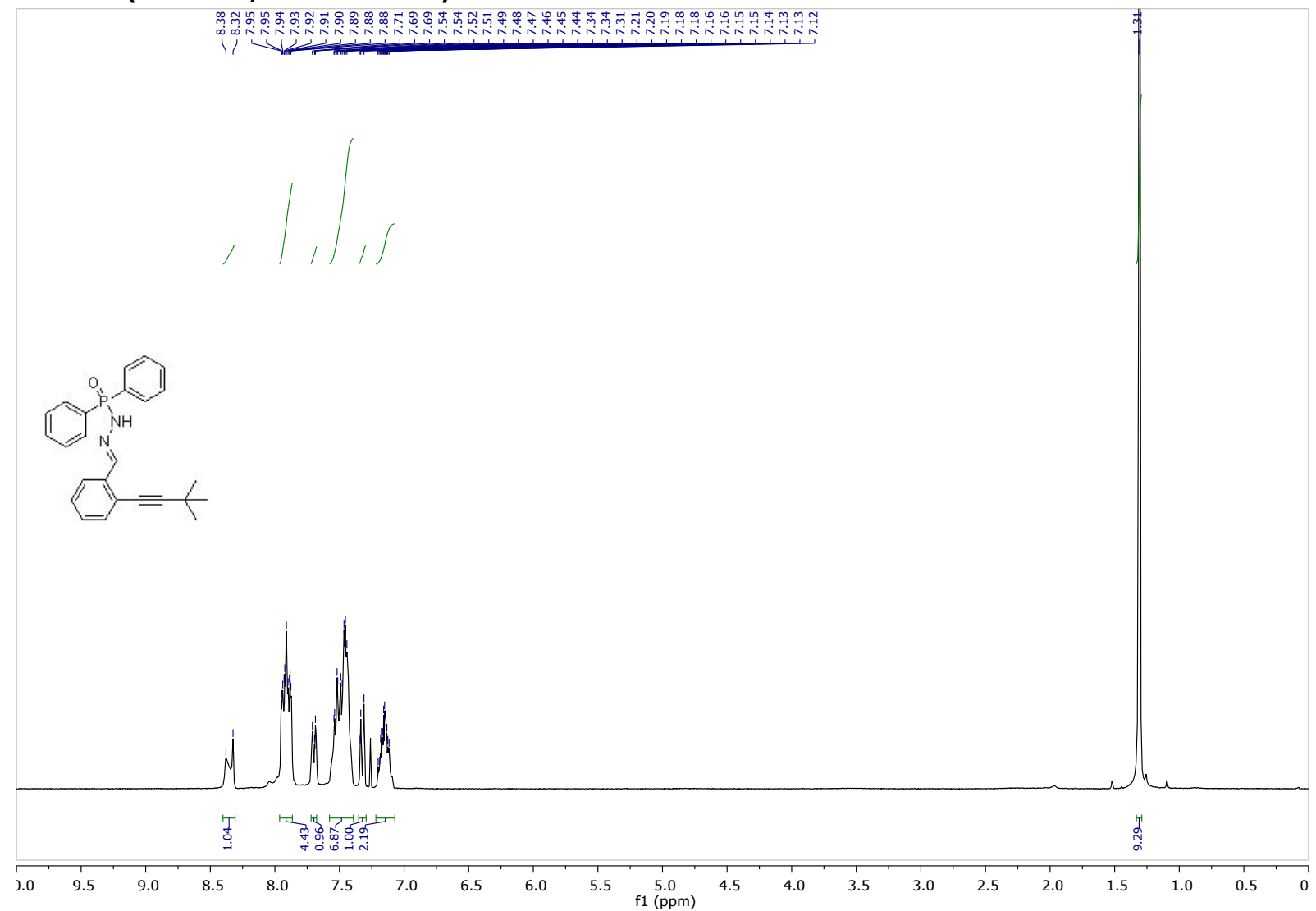

${ }^{13} \mathrm{C}\left\{{ }^{1} \mathrm{H}\right\}$ NMR (75 MHz, Chloroform-d)

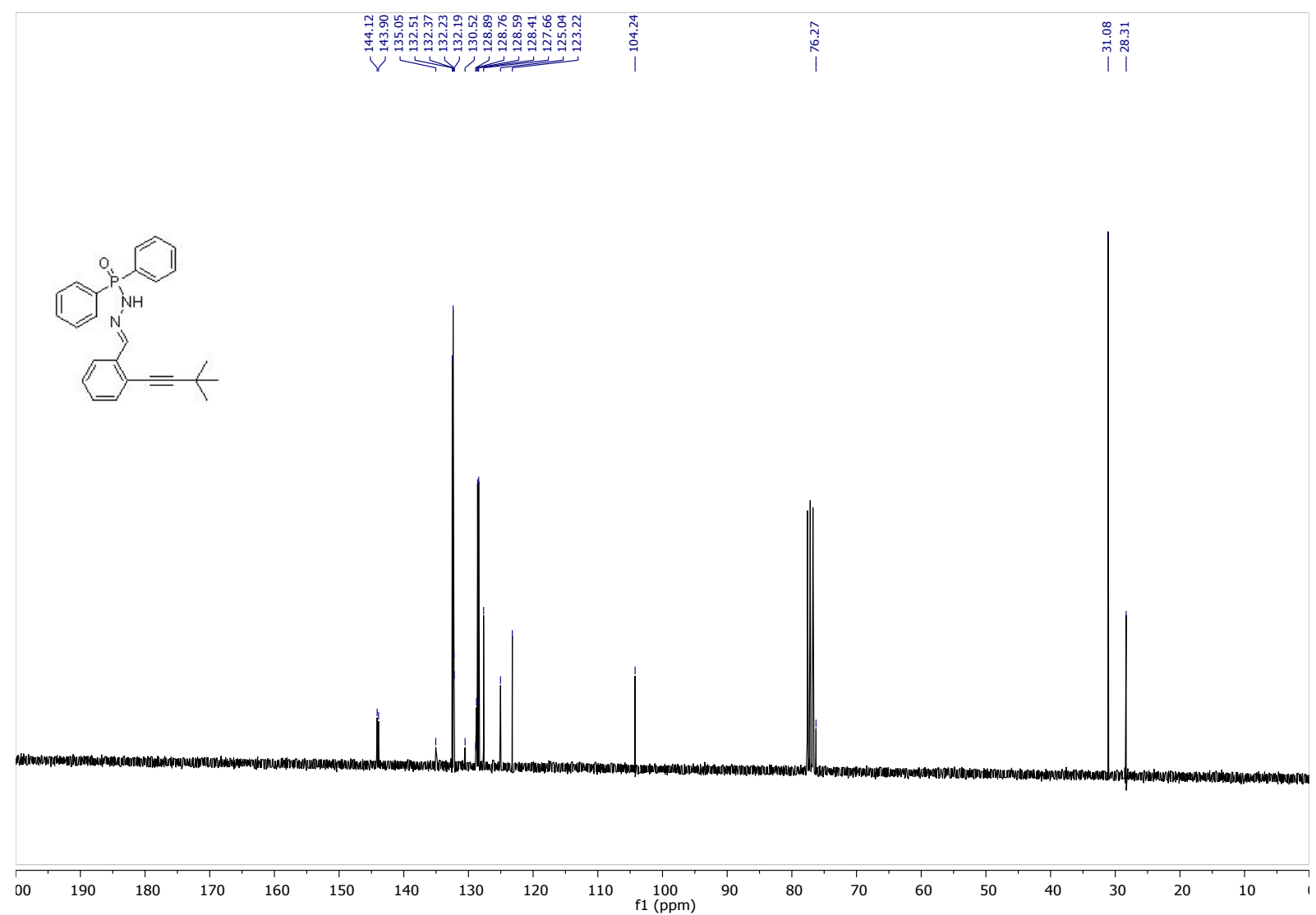


31P NMR (162 MHz, Chloroform-d)
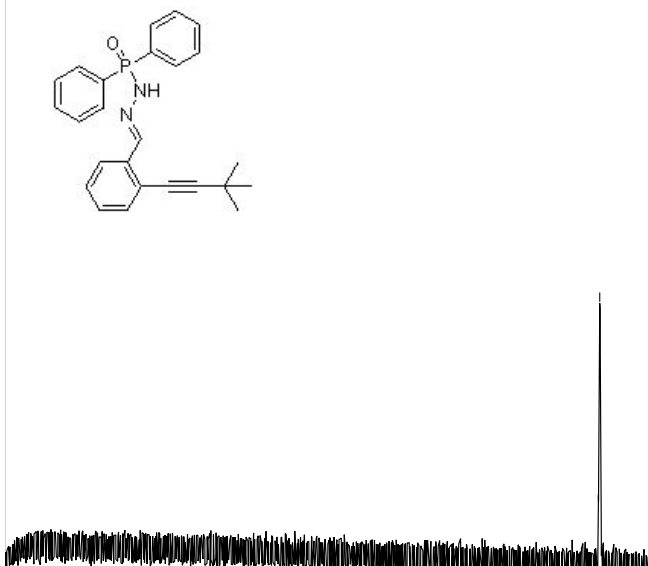

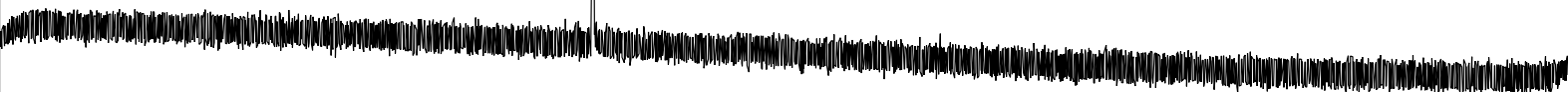

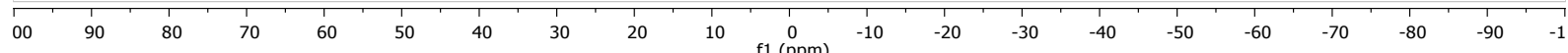


Compound 1h: P,P-diphenyl-N'-(3,4,5-trimethoxy-2-(phenylethynyl)benzylidene)phosphinic hydrazide

${ }^{1} \mathrm{H}$ NMR (300 MHz, Chloroform-d)

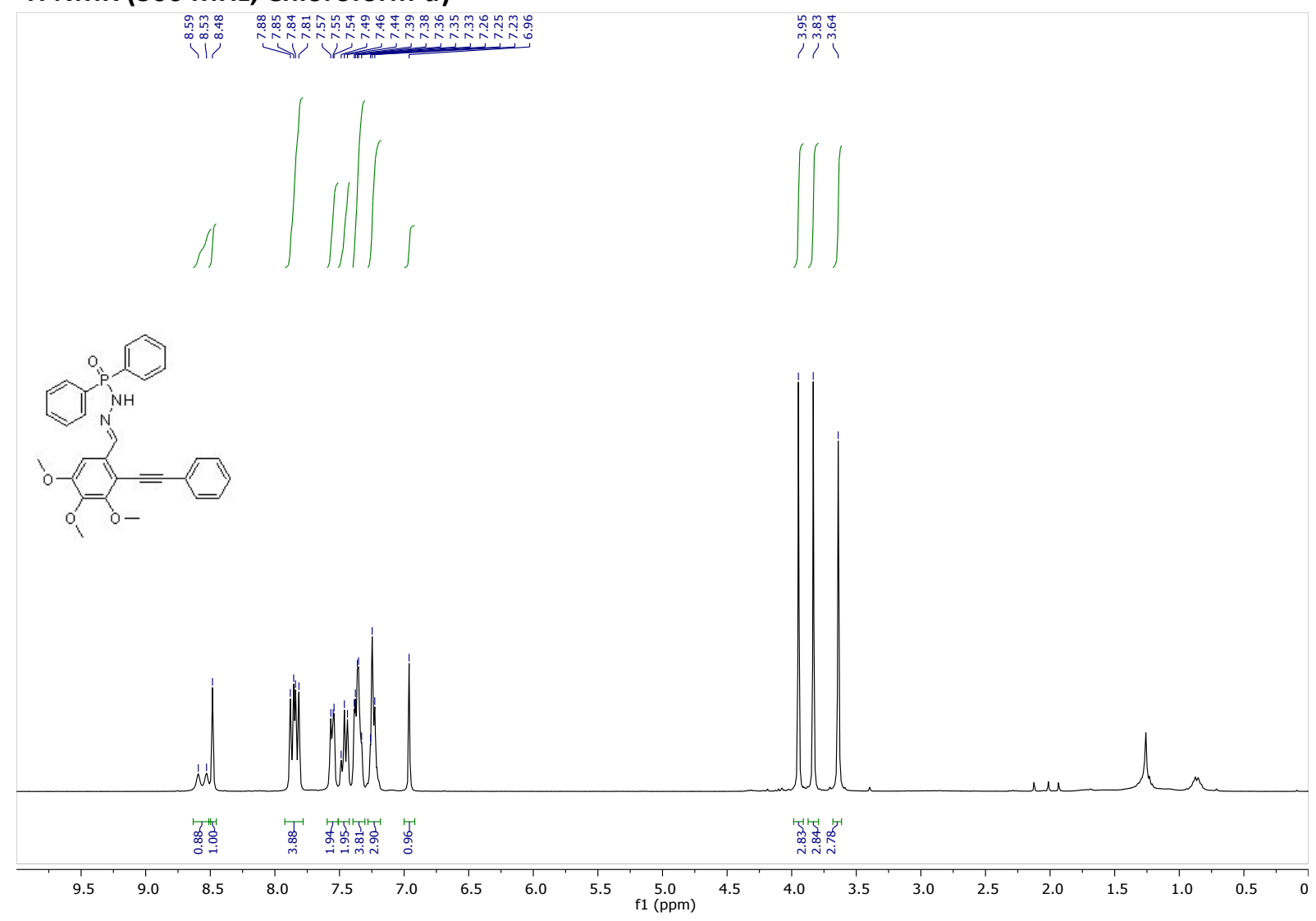

${ }^{13} \mathrm{C}\left\{{ }^{1} \mathrm{H}\right\}$ NMR (75 MHz, Chloroform-d) (JMOD) spectrum

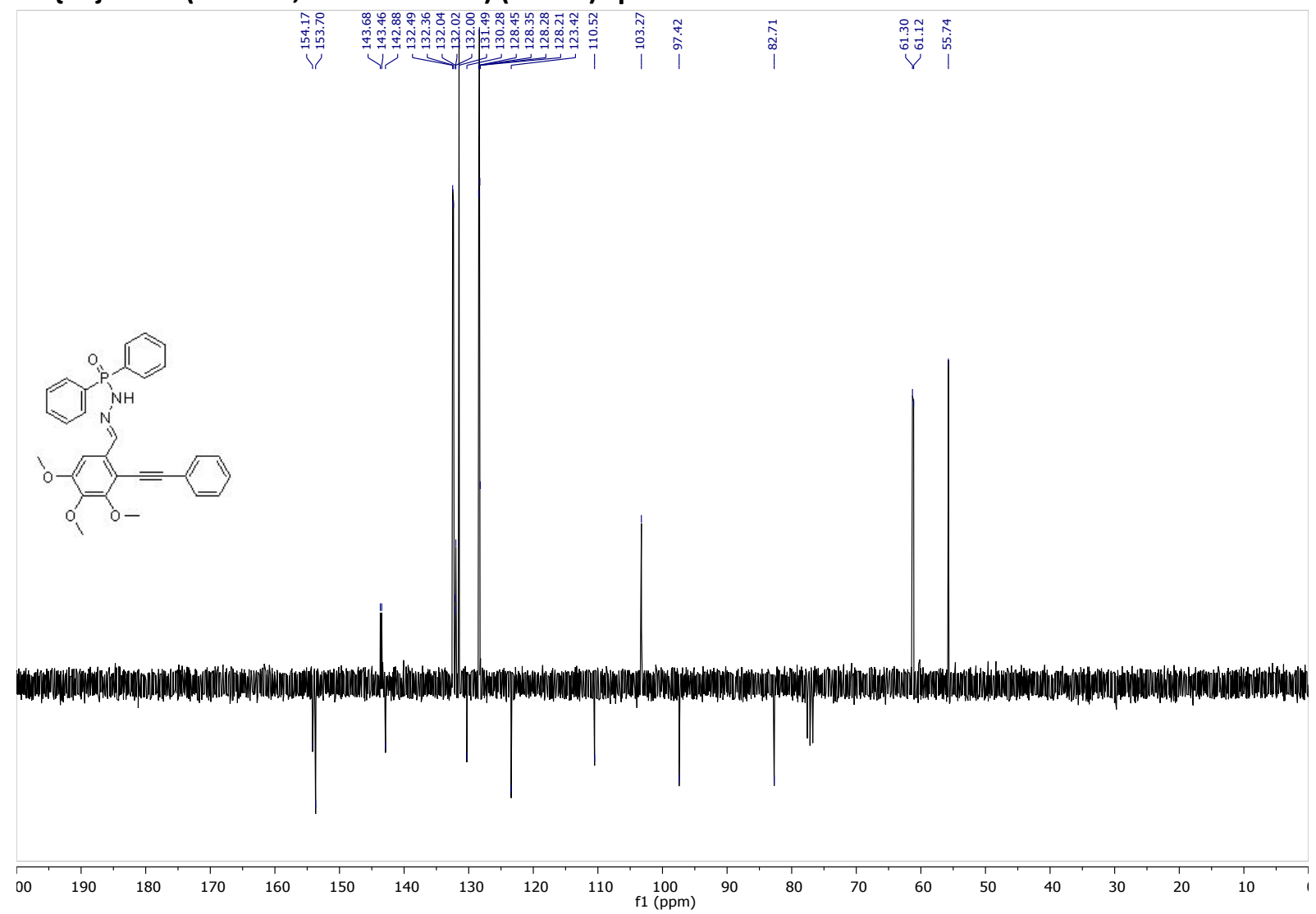


31P NMR (121 MHz, Chloroform-d)

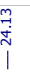

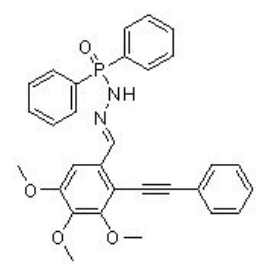

00

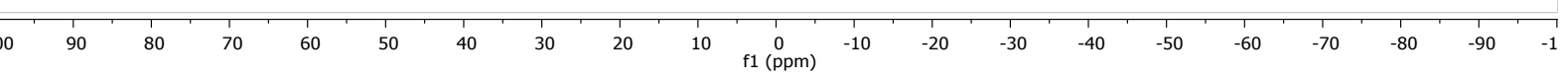

S25 
Compound 1i: N'-(5-fluoro-2-(phenylethynyl)benzylidene)-P,P-diphenylphosphinic hydrazide ${ }^{1} \mathrm{H}$ NMR (300 MHz, Chloroform-d)

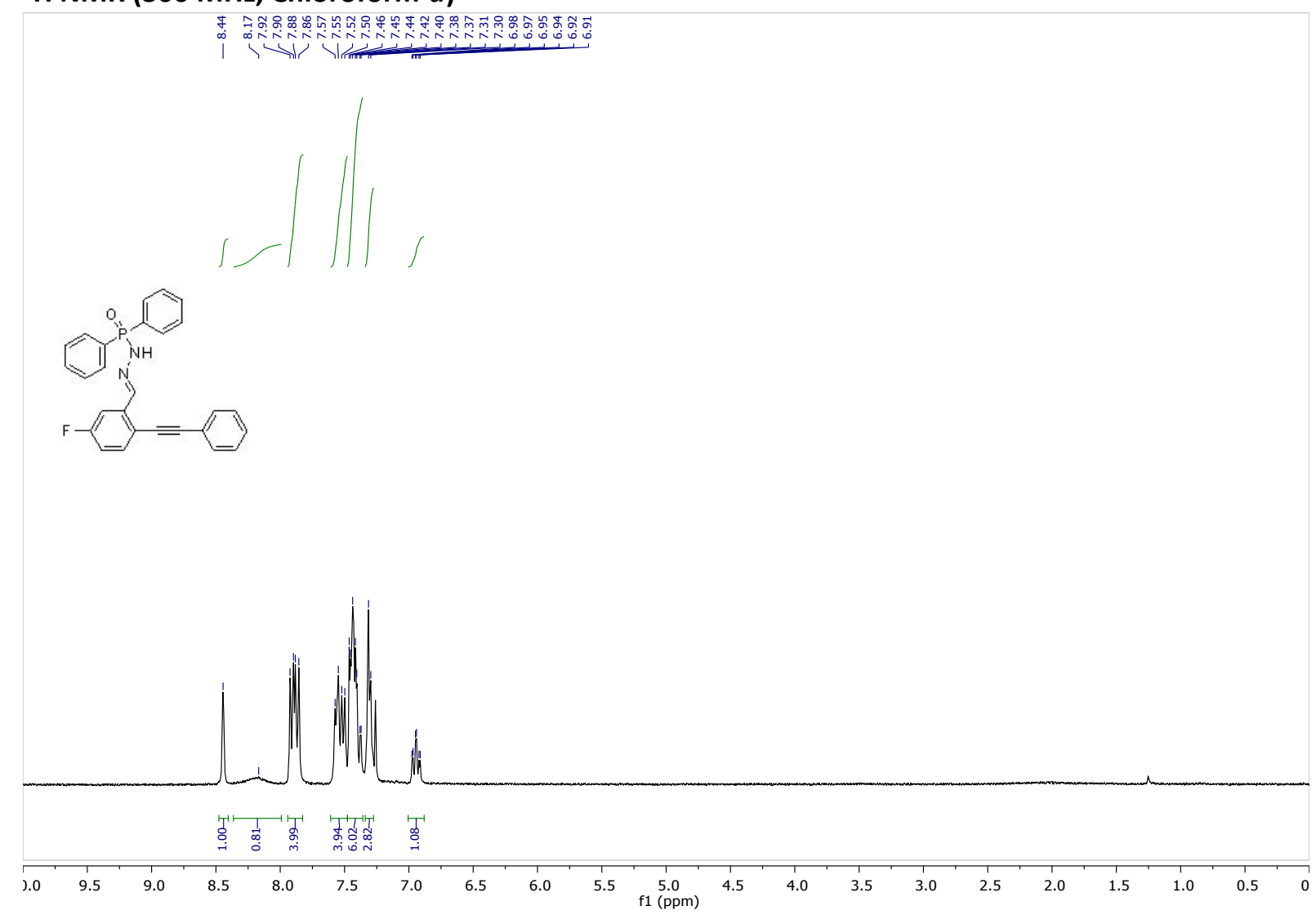

${ }^{13} \mathrm{C}\left\{{ }^{1} \mathrm{H}\right\}$ NMR (101 MHz, Chloroform-d)

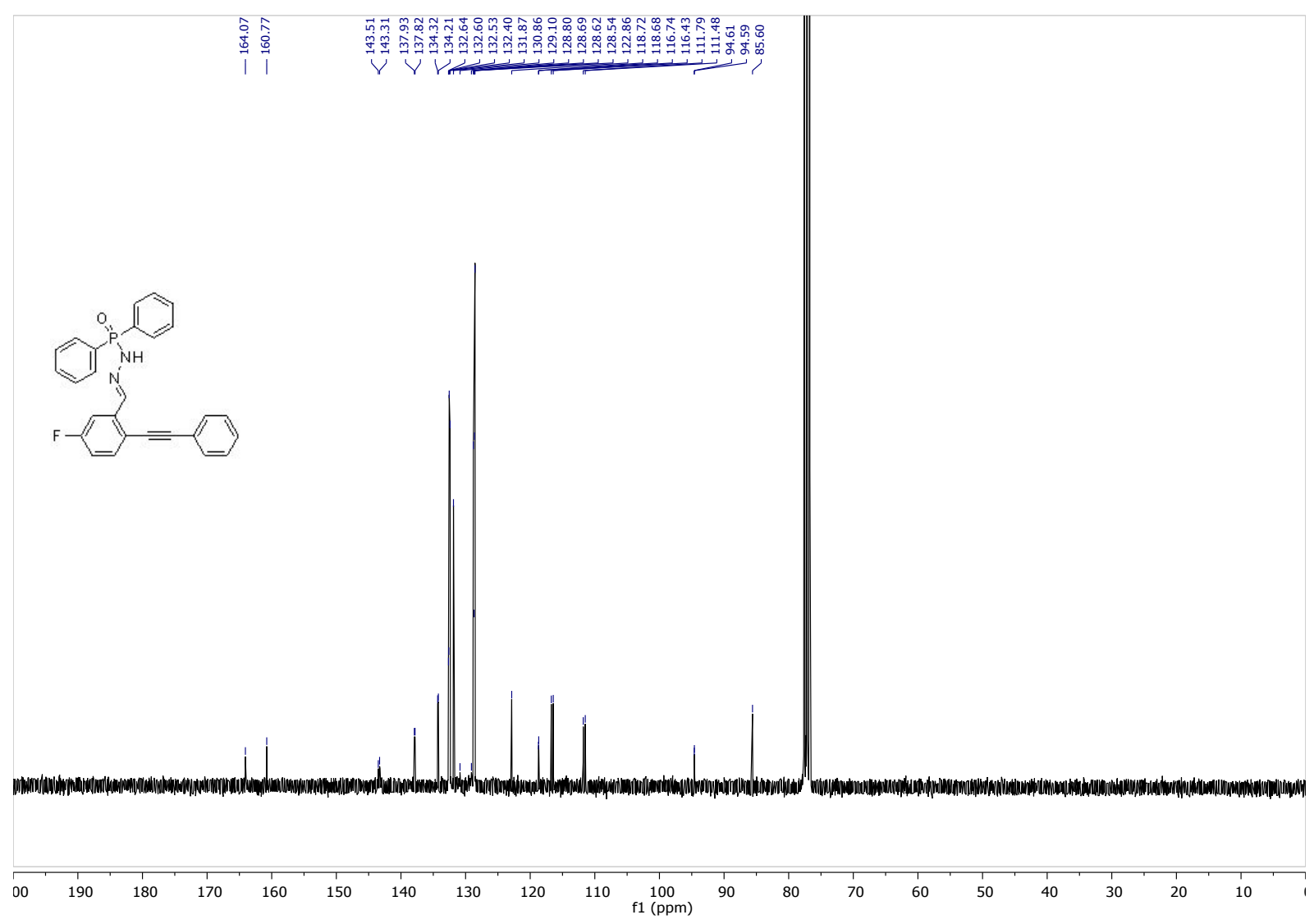

S26 
31P NMR (121 MHz, Chloroform-d)
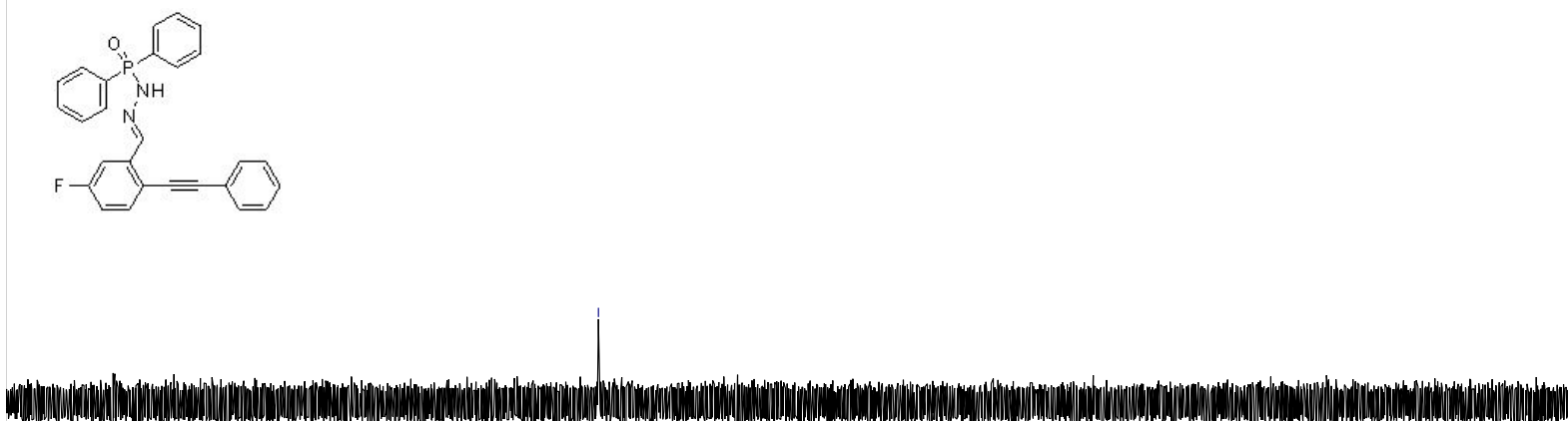

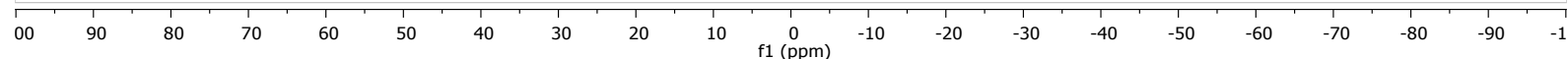

${ }^{19} \mathrm{~F}$ NMR (282 MHz, Chloroform-d)

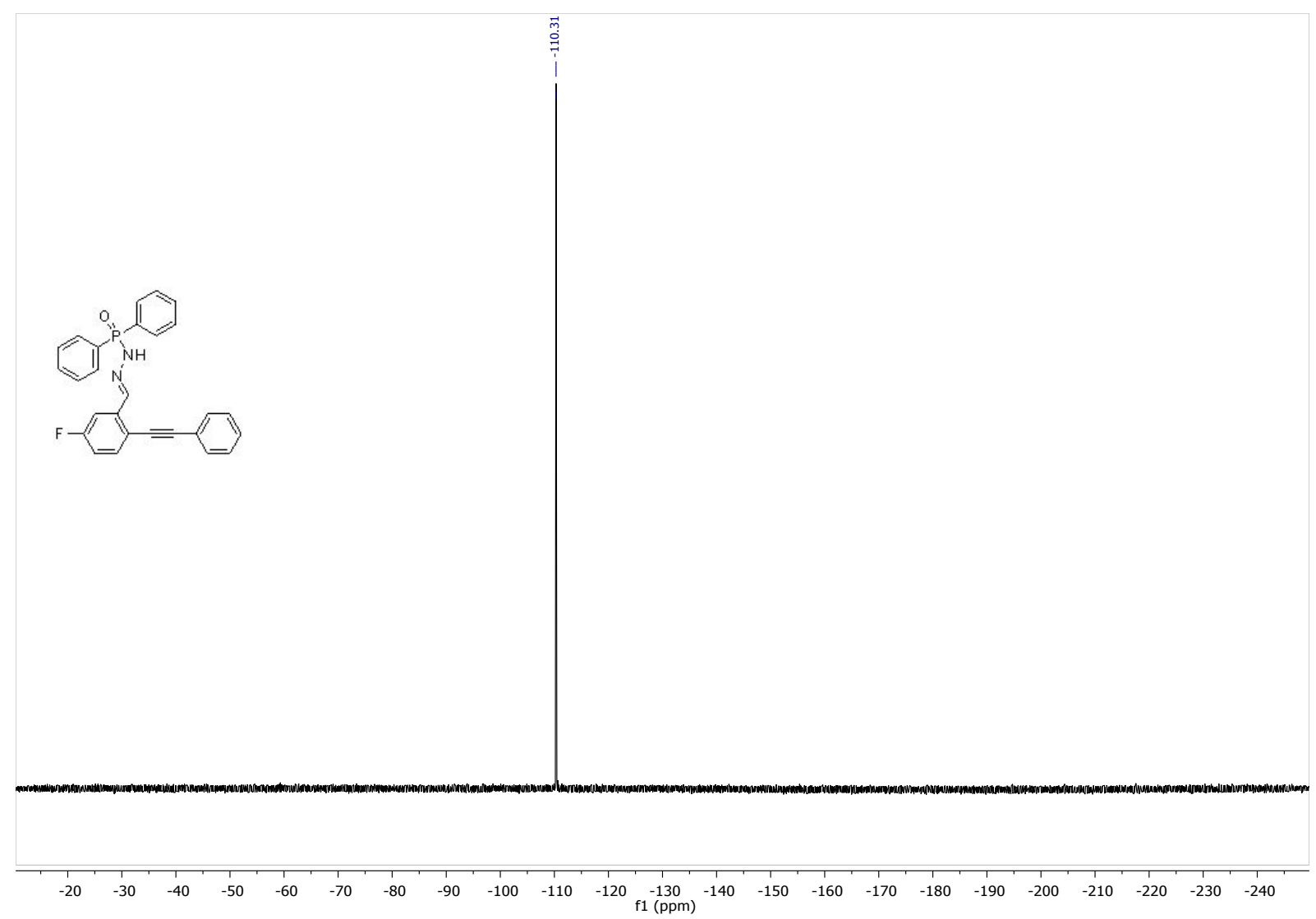


Compound 1j: N'-(5-chloro-2-(phenylethynyl)benzylidene)-P,P-diphenylphosphinic hydrazide ${ }^{1} \mathrm{H}$ NMR (300 MHz, Chloroform-d)

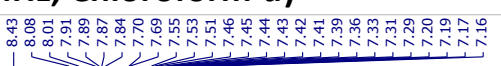

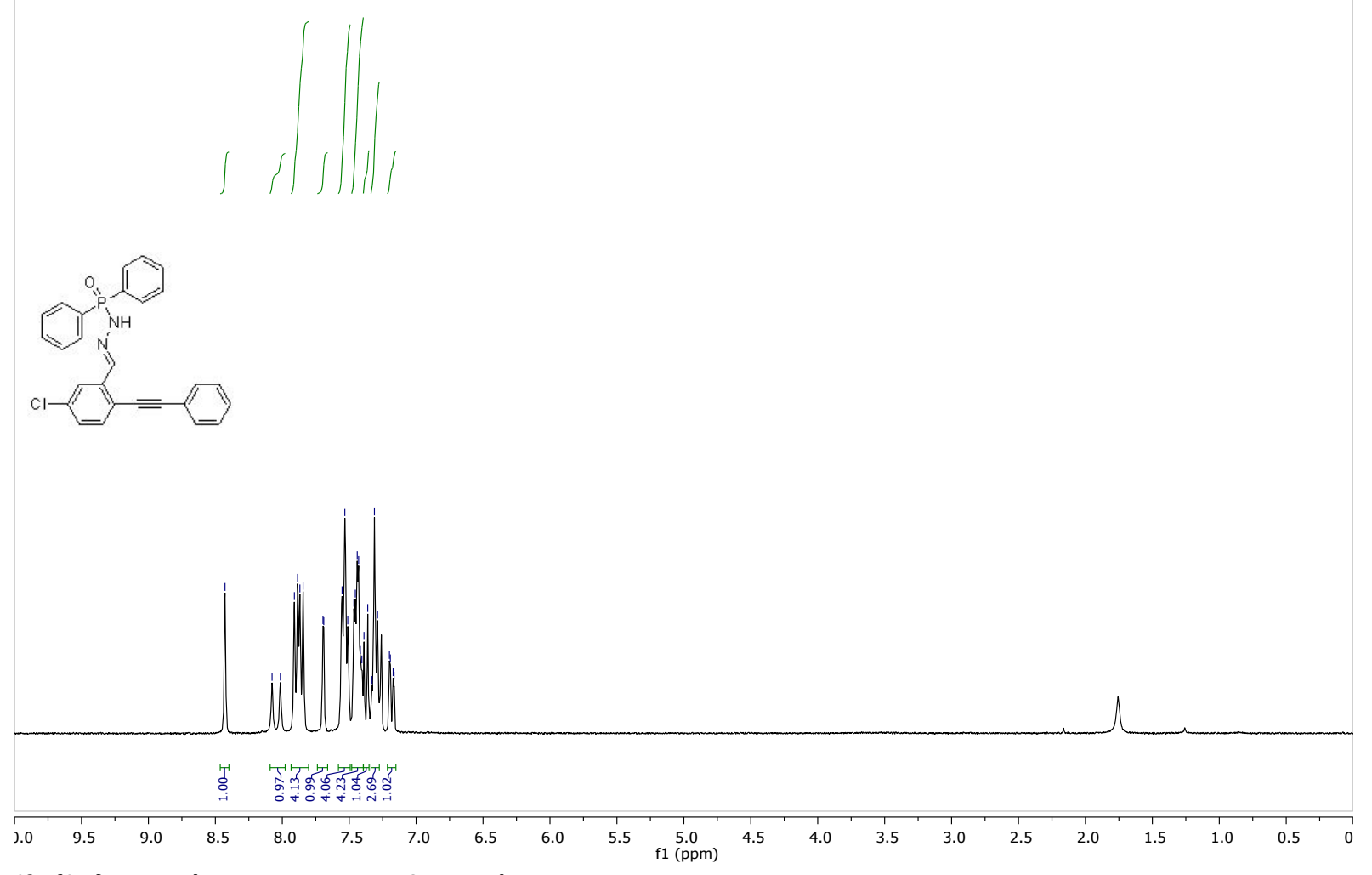

${ }^{13} \mathrm{C}\left\{{ }^{1} \mathrm{H}\right\}$ NMR (75 MHz, Chloroform- $d$ )

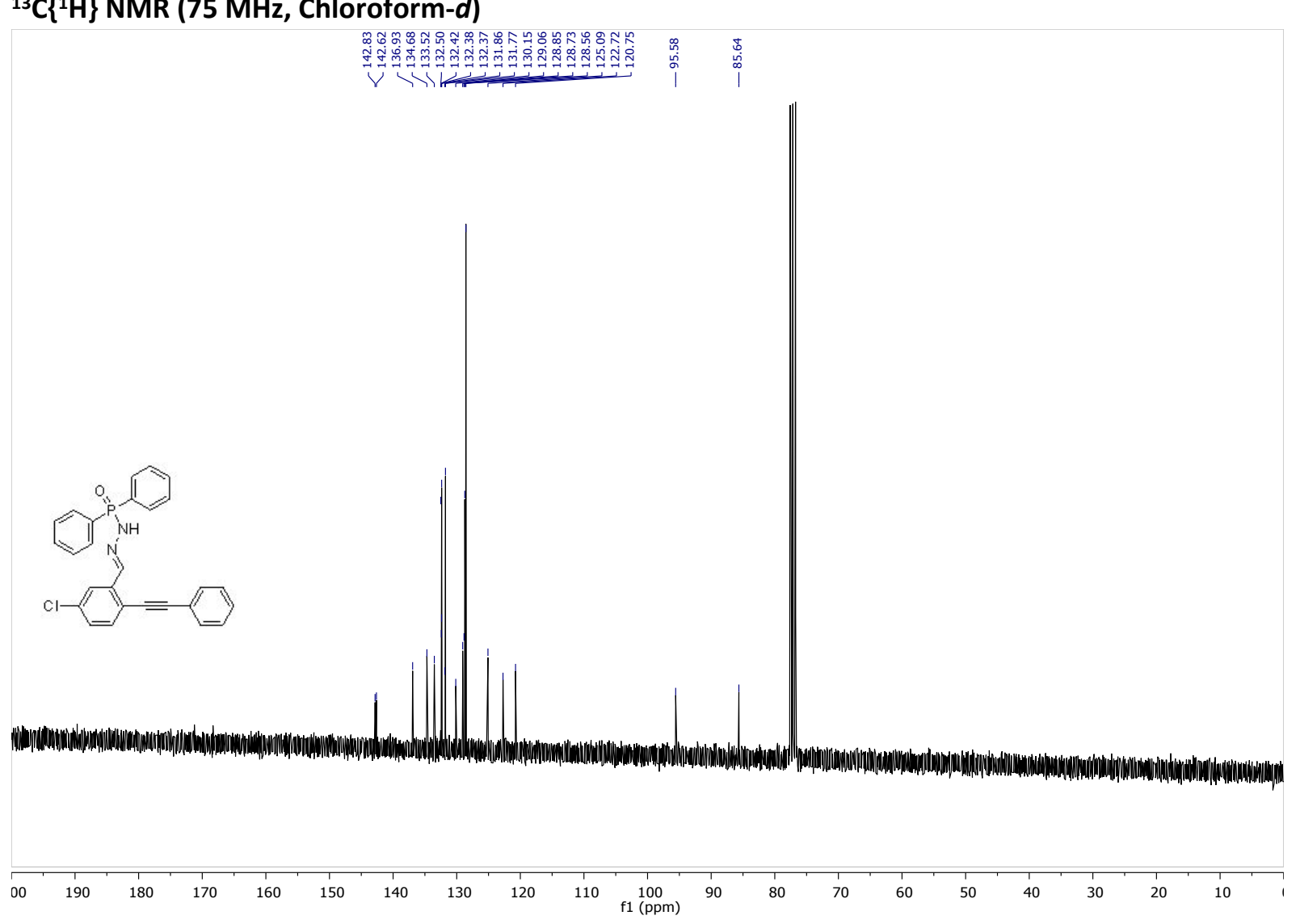

S28 
31P NMR (121 MHz, Chloroform-d)

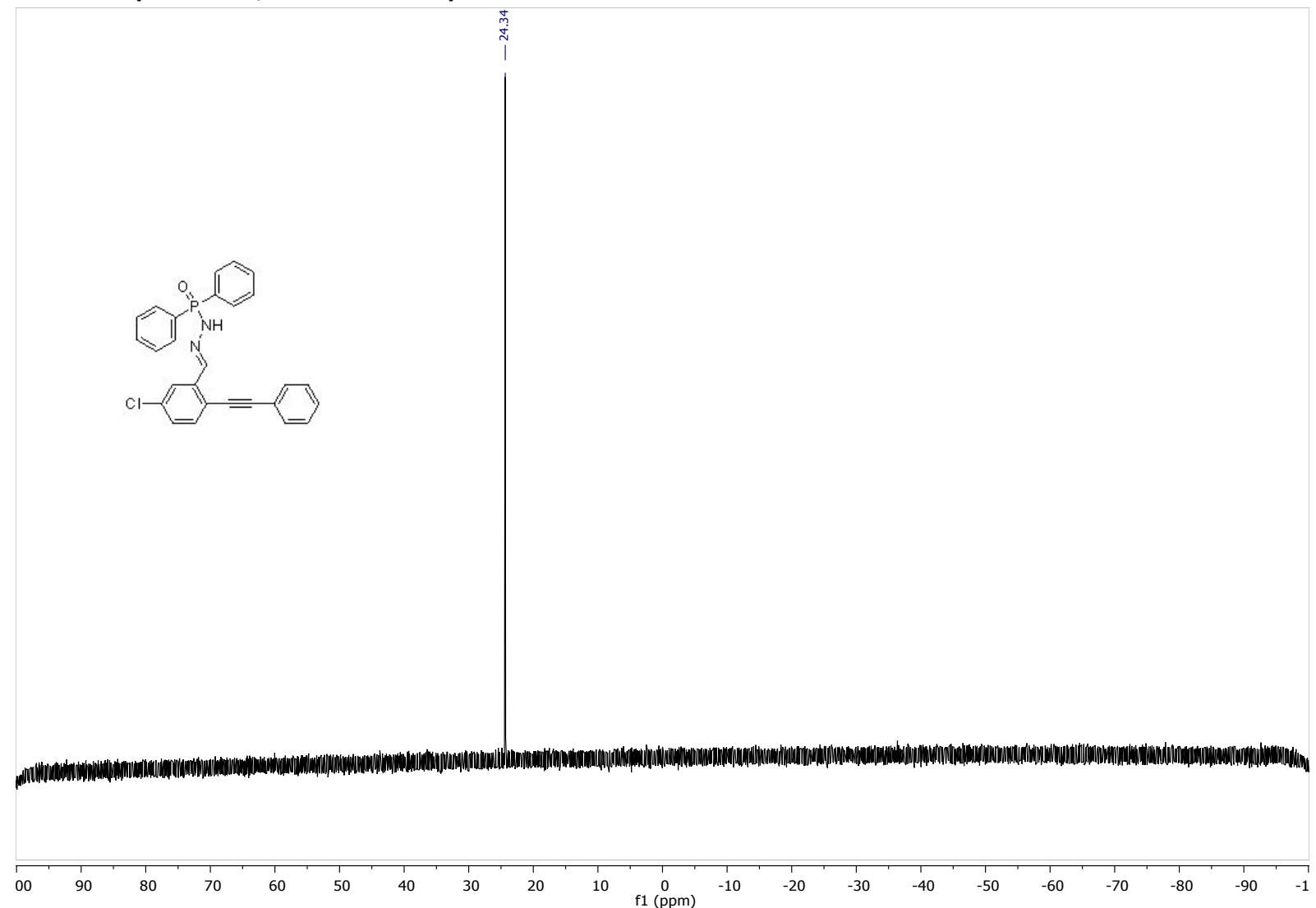


Compound 1k: N'-(5-bromo-2-(phenylethynyl)benzylidene)-P,P-diphenylphosphinic hydrazide ${ }^{1} \mathrm{H}$ NMR (300 MHz, Chloroform-d)

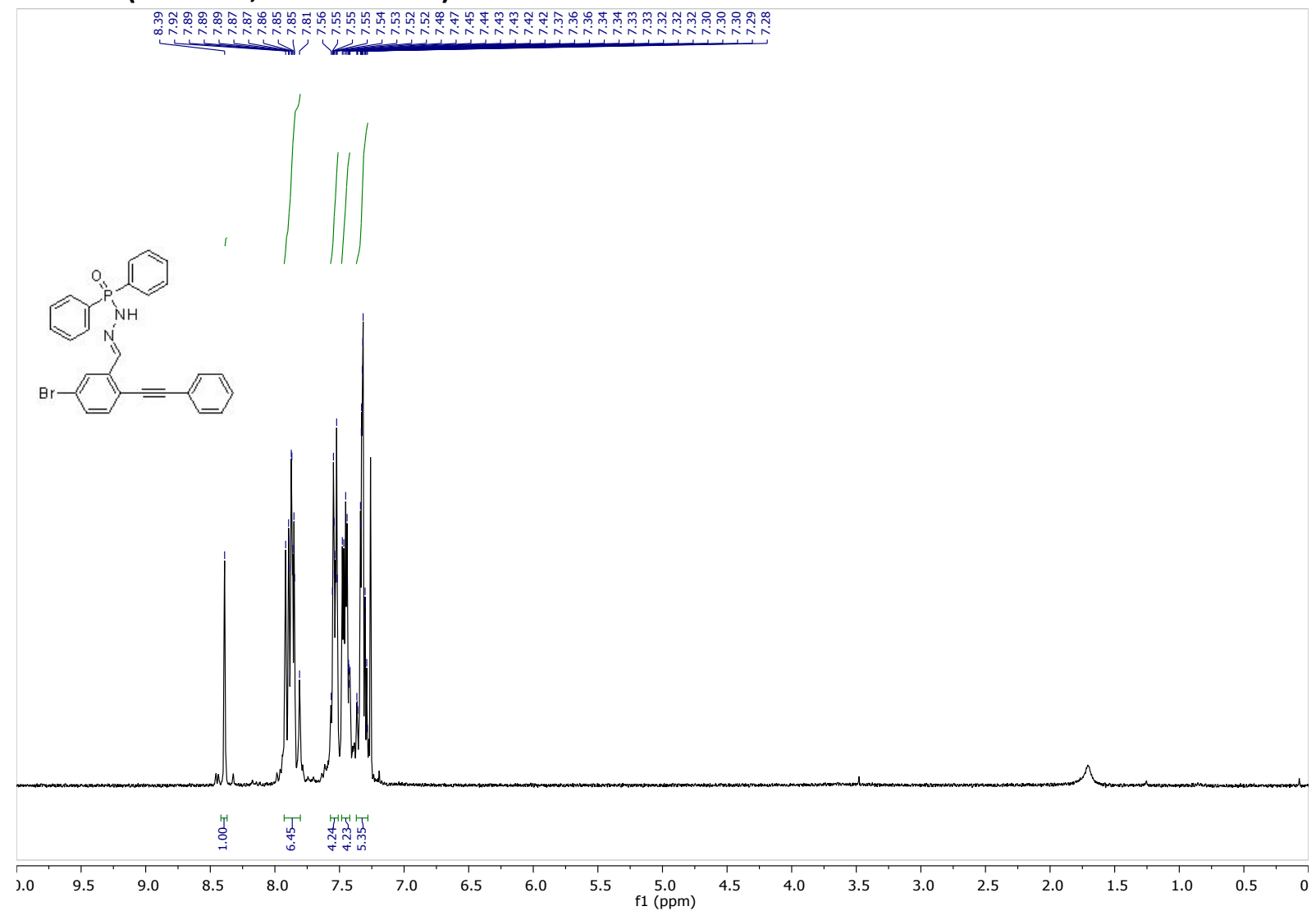

${ }^{13} \mathrm{C}\left\{{ }^{1} \mathrm{H}\right\}$ NMR (75 MHz, Chloroform-d)

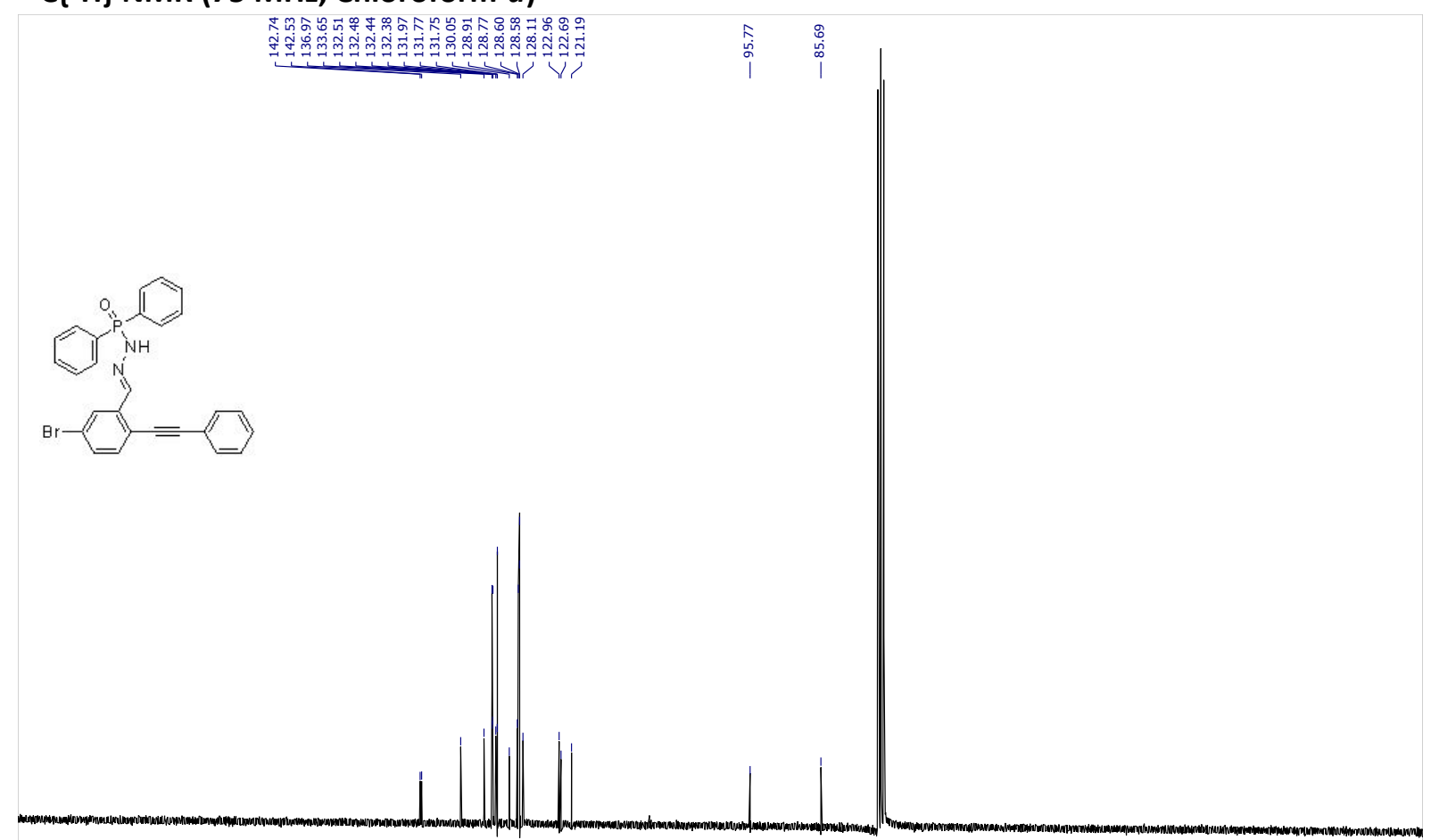

Do

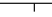

160

150

13

$120 \quad 110$

100
$\mathrm{f} 1(\mathrm{ppm})$

$90 \quad 80$

0

(60)

$\begin{array}{lllll}50 & 40 & 30 & 20 & 10\end{array}$ 
${ }^{31}$ P NMR (121 MHz, Chloroform-d)

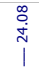

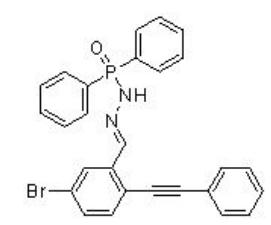

Wy

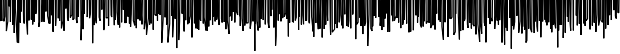

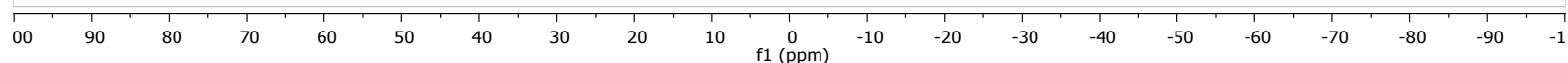


Compound 11: P,P-diphenyl-N'-((2-(phenylethynyl)cyclohex-1-en-1-yl)methylene)phosphinic hydrazide

${ }^{1}$ H NMR (300 MHz, Chloroform-d)

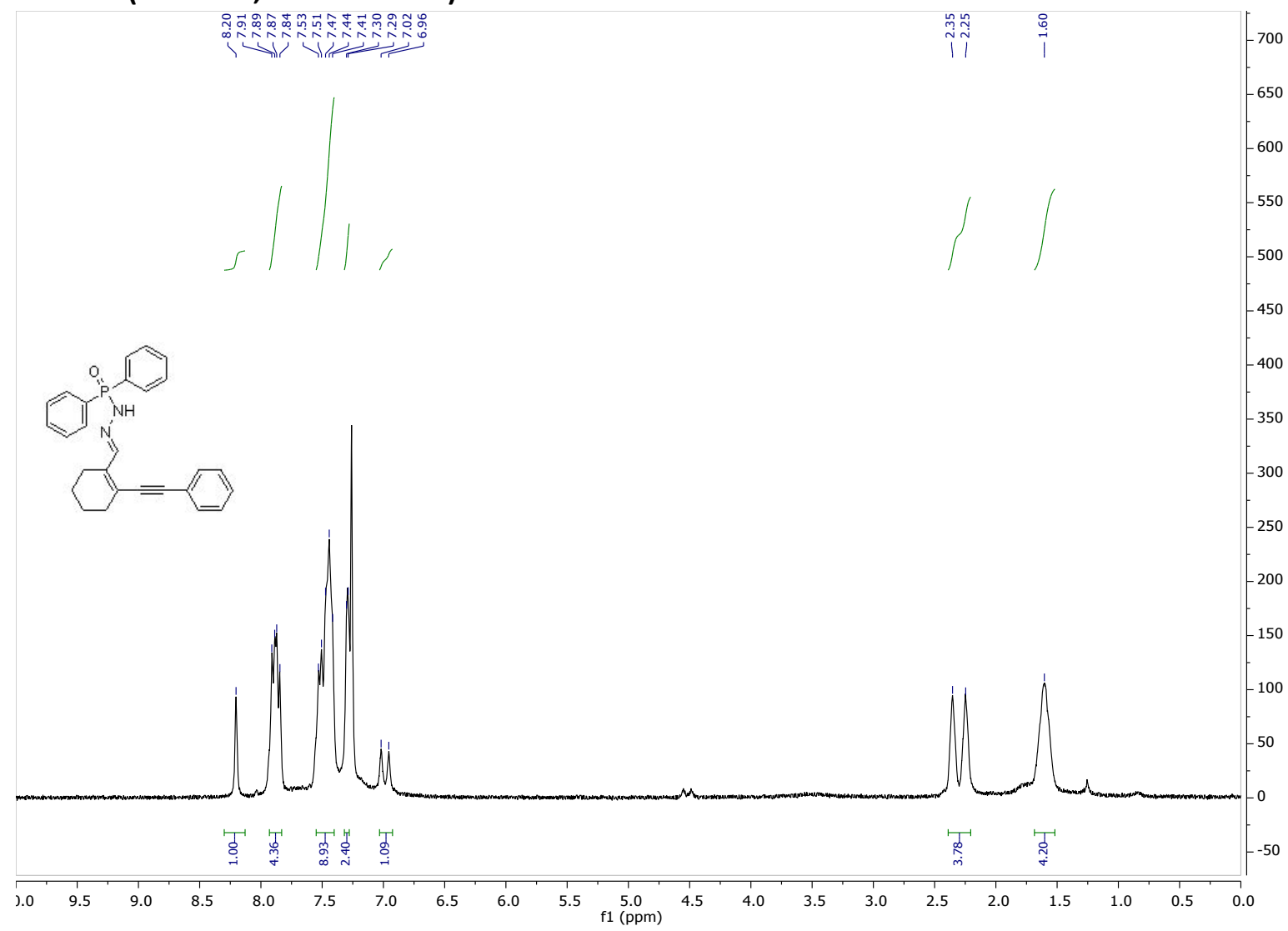

${ }^{13} \mathrm{C}\left\{{ }^{1} \mathrm{H}\right\}$ NMR (75 MHz, Chloroform-d)

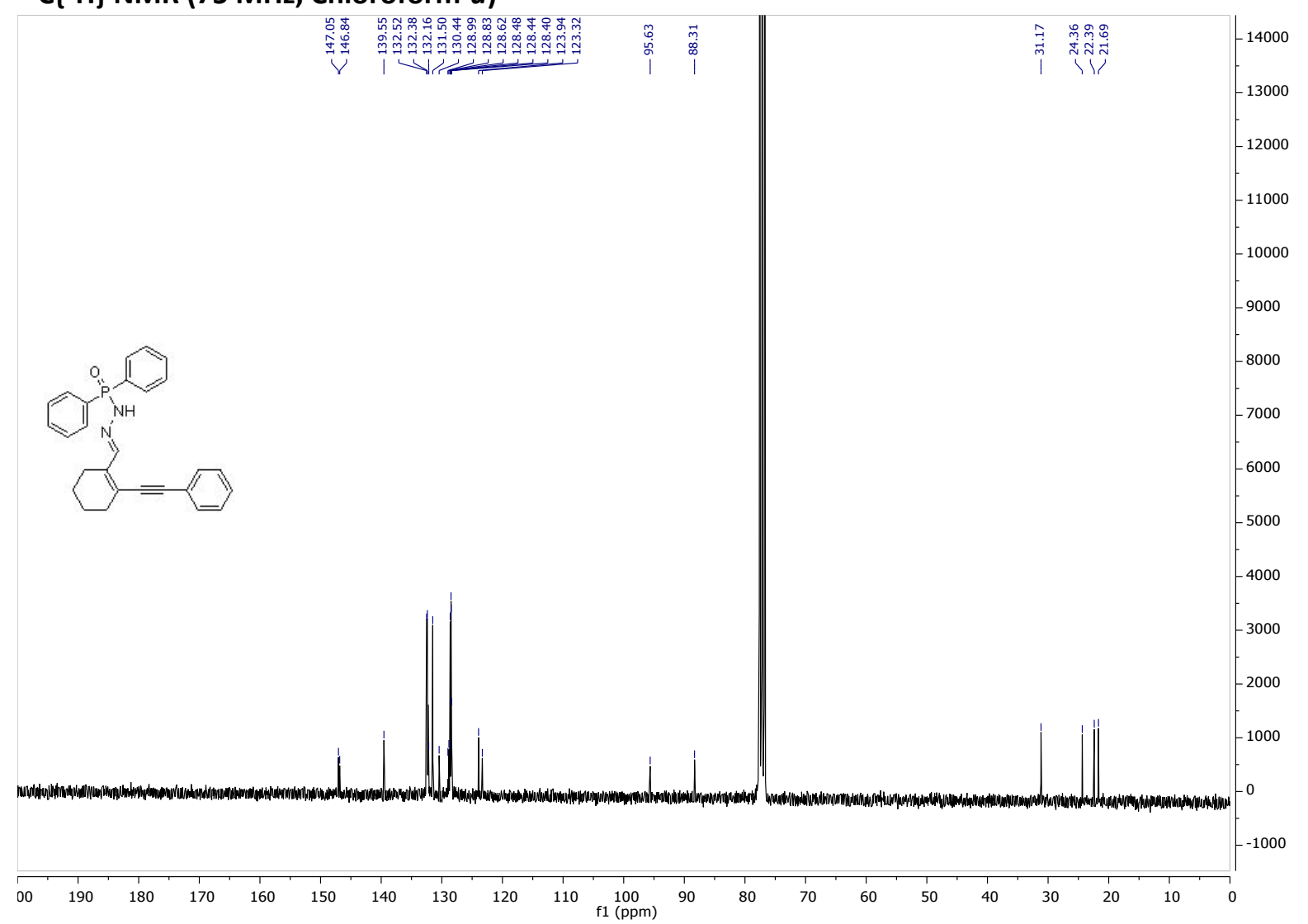


31P NMR (121 MHz, Chloroform-d)

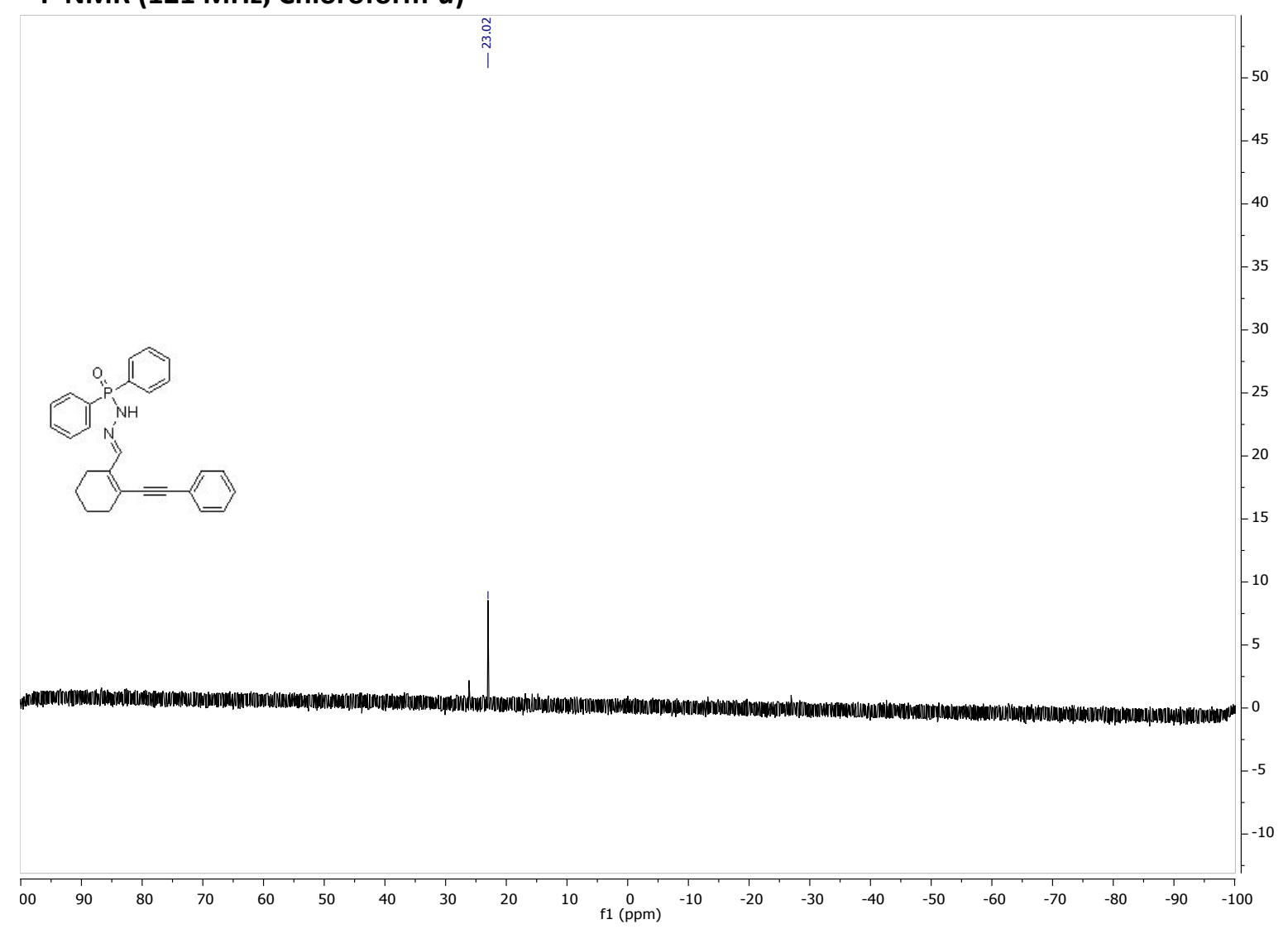


Compound 1m: N'-((1-methyl-2-(phenylethynyl)-1H-indol-3-yl)methylene)-P,P-diphenylphosphinic hydrazide

${ }^{1} \mathrm{H}$ NMR (300 MHz, Chloroform-d)

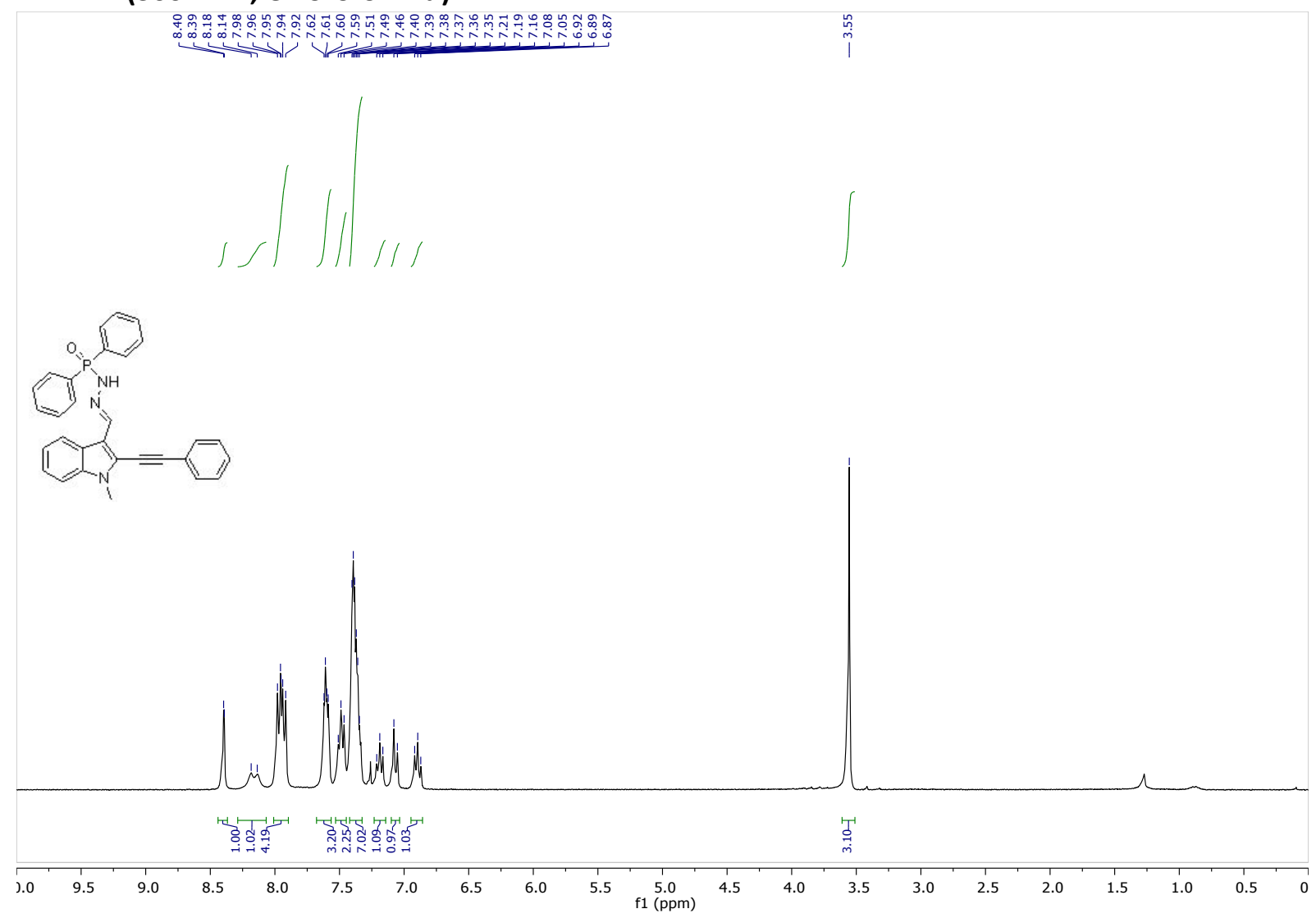

${ }^{13} \mathrm{C}\left\{{ }^{1} \mathrm{H}\right\}$ NMR (75 MHz, Chloroform-d) (JMOD) spectrum

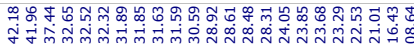

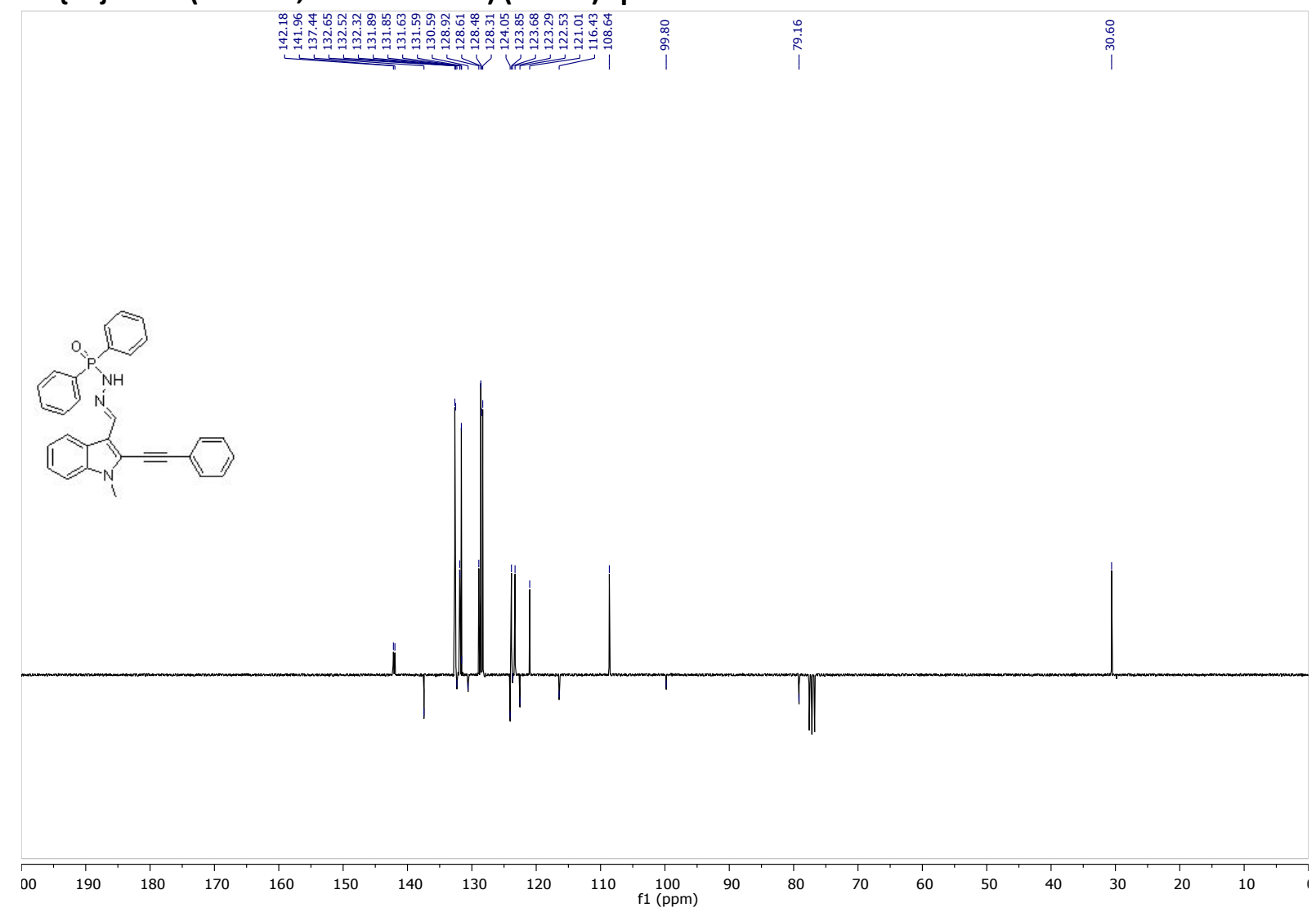


31P NMR (121 MHz, Chloroform-d)
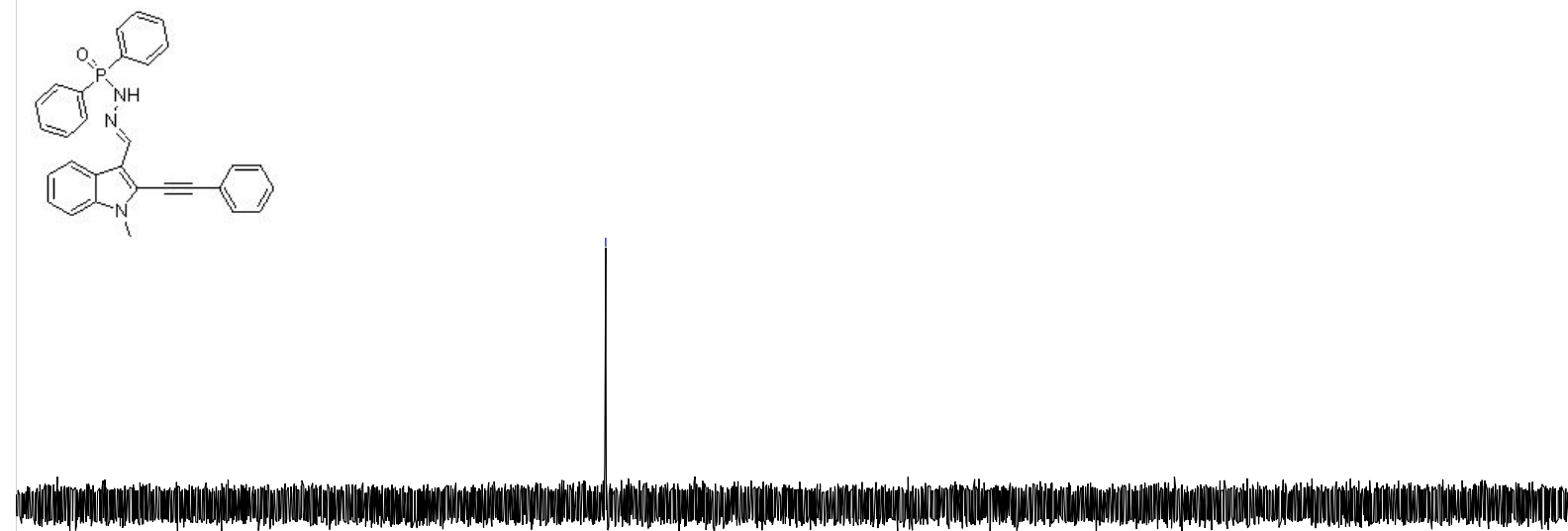

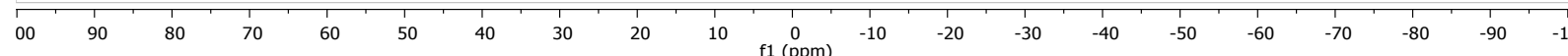


Compound 1n: P,P-diphenyl-N'-((2-(phenylethynyl)benzofuran-3-yl)methylene)phosphinic hydrazide

${ }^{1} \mathrm{H}$ NMR (300 MHz, Chloroform-d)

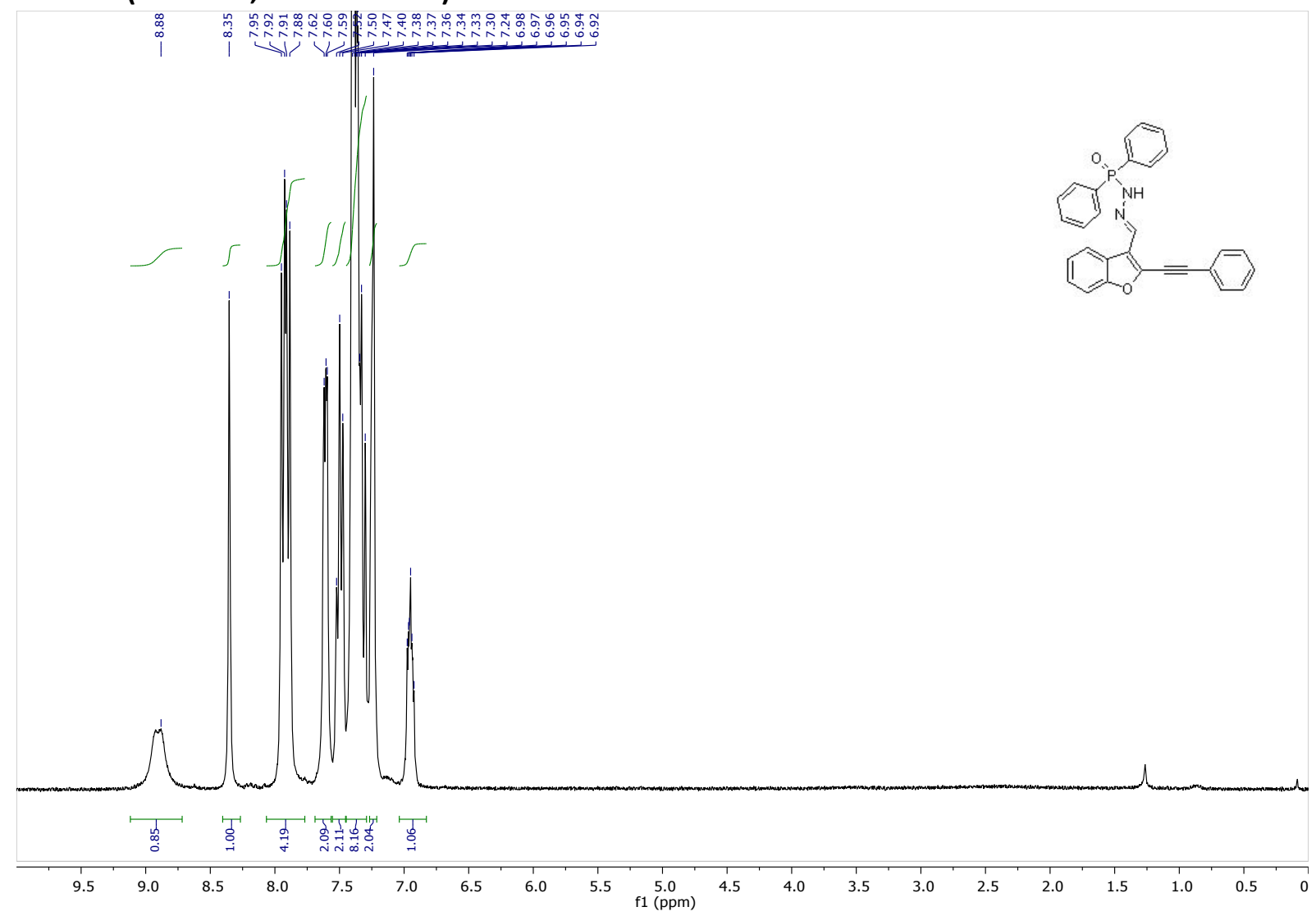

${ }^{13} \mathrm{C}\left\{{ }^{1} \mathrm{H}\right\}$ NMR (75 MHz, Chloroform-d) (JMOD) spectrum

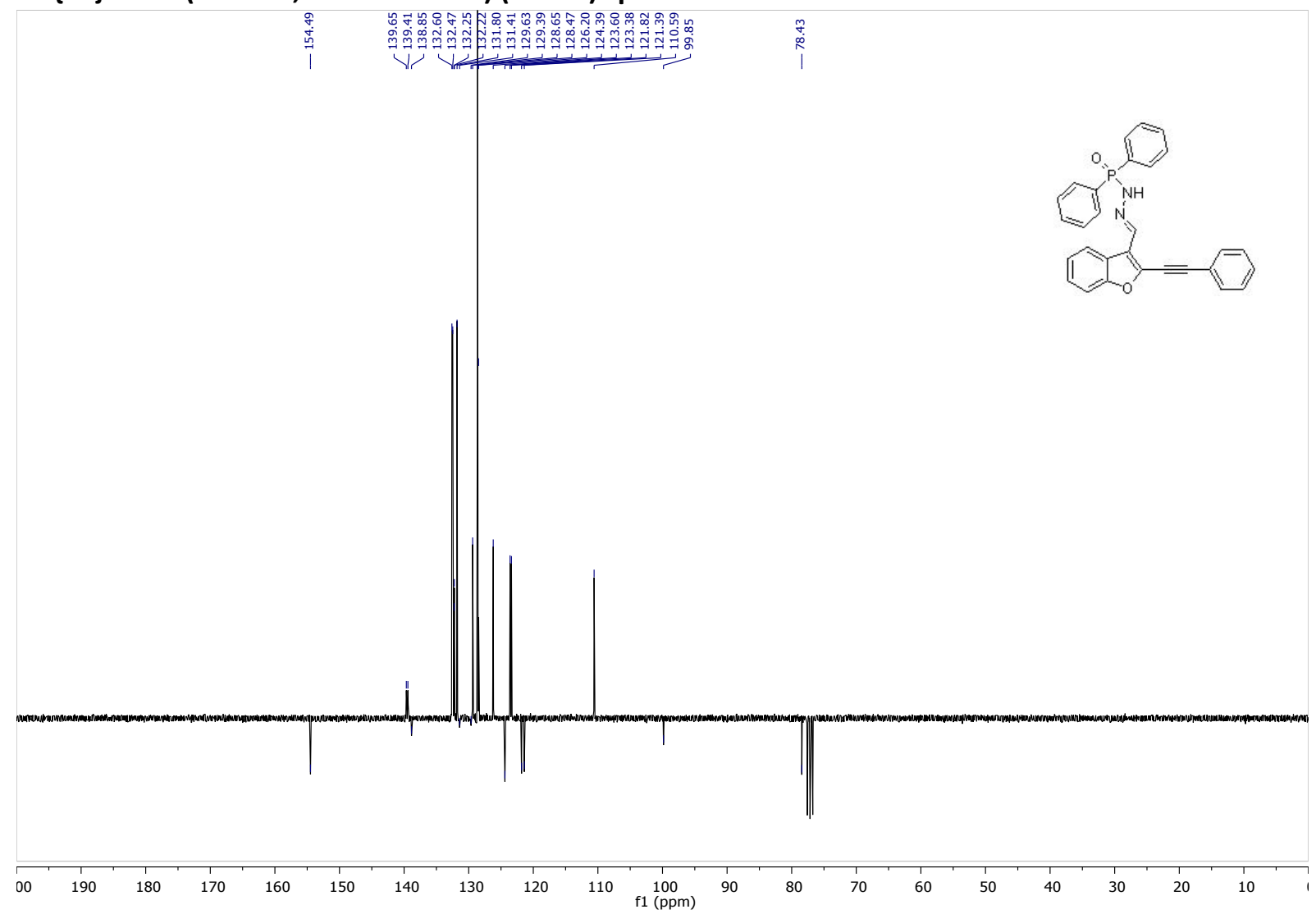


31P NMR (121 MHz, Chloroform-d)

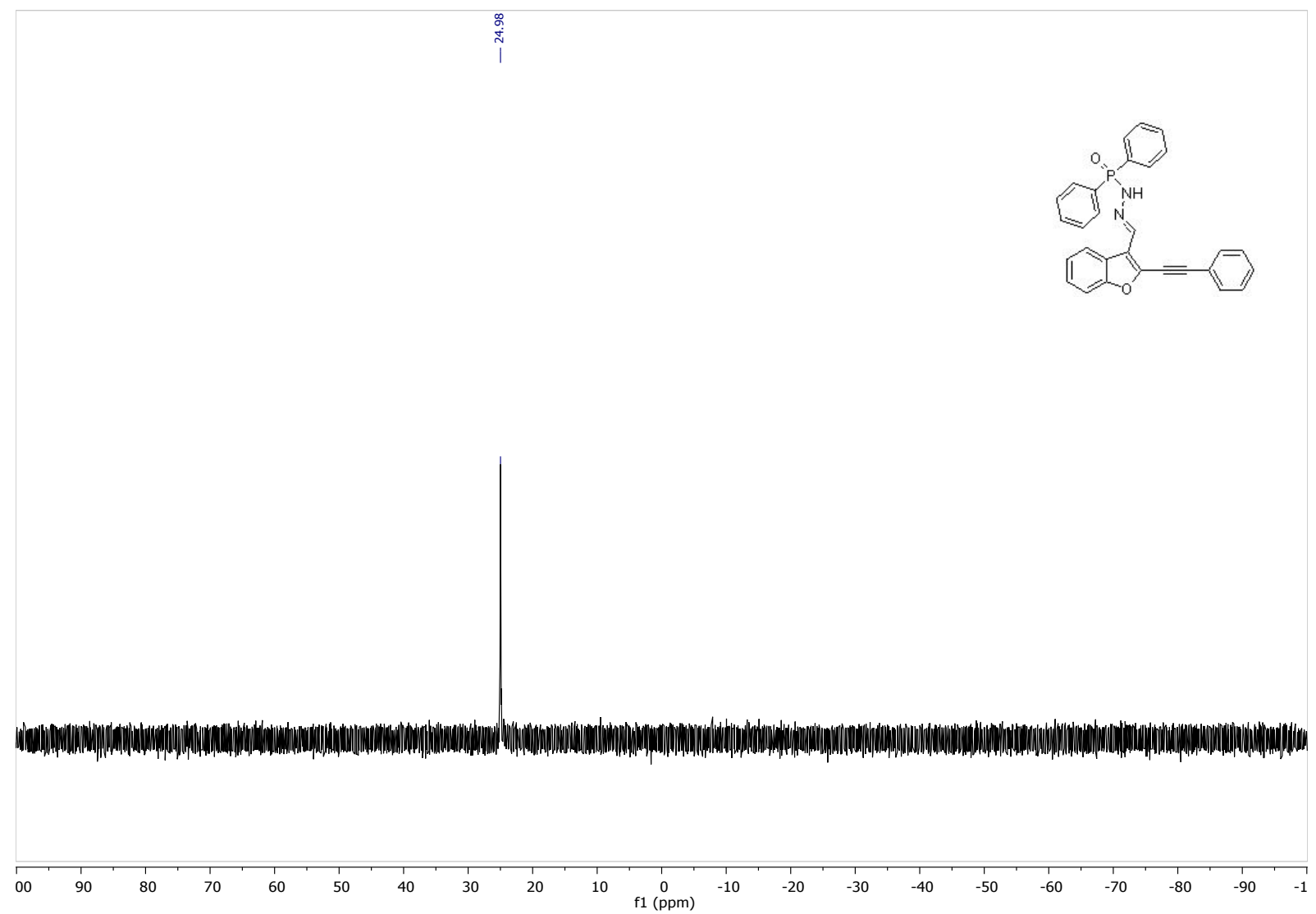


Compound 10: P,P-diphenyl-N'-((2-(phenylethynyl)quinolin-3-yl)methylene)phosphinic hydrazide ${ }^{1} \mathrm{H}$ NMR (400 MHz, Chloroform-d)

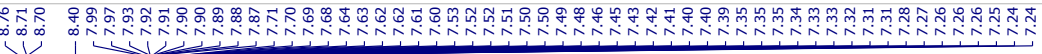

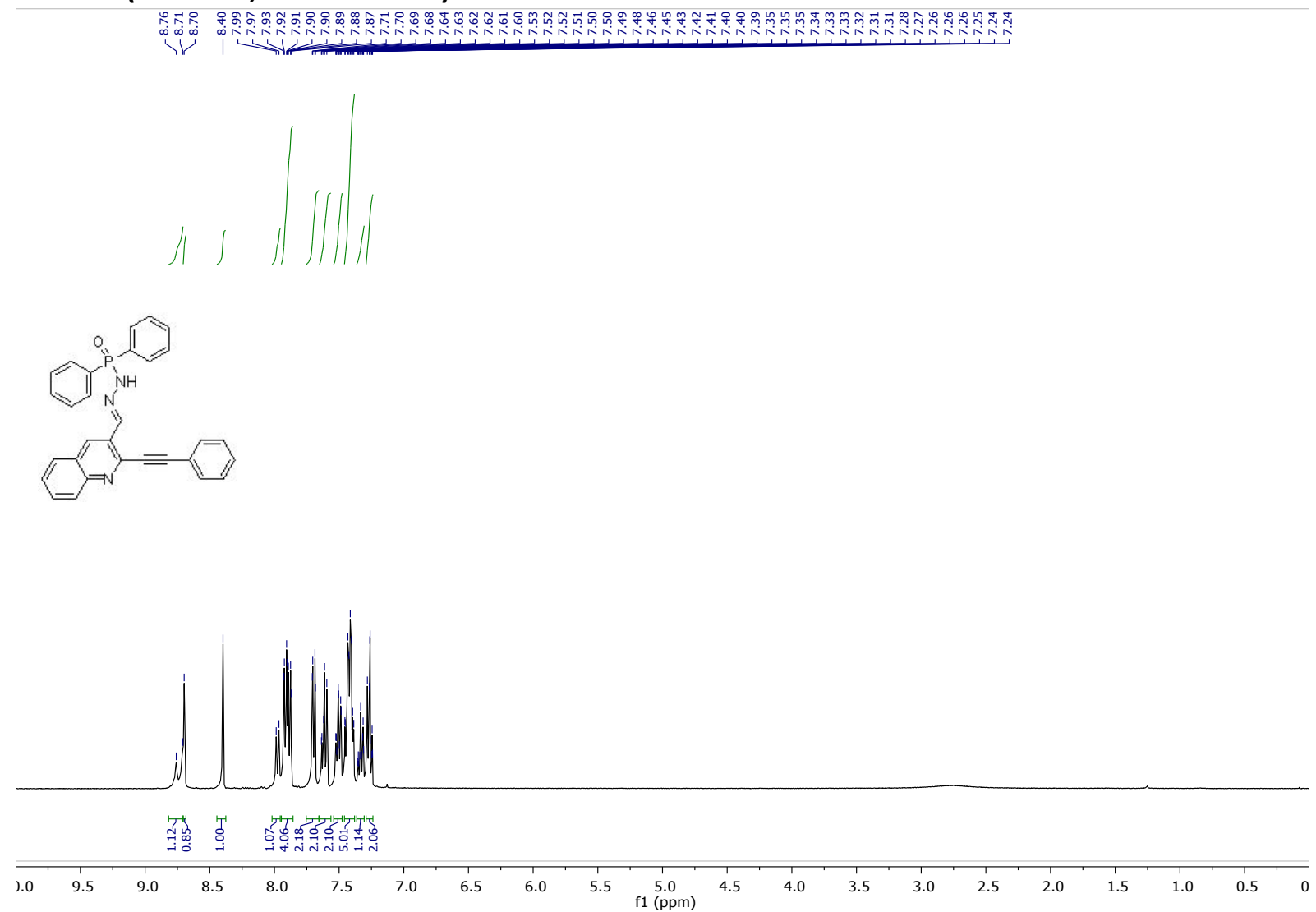

${ }^{13} \mathrm{C}\left\{{ }^{1} \mathrm{H}\right\}$ NMR (101 MHz, Chloroform-d)

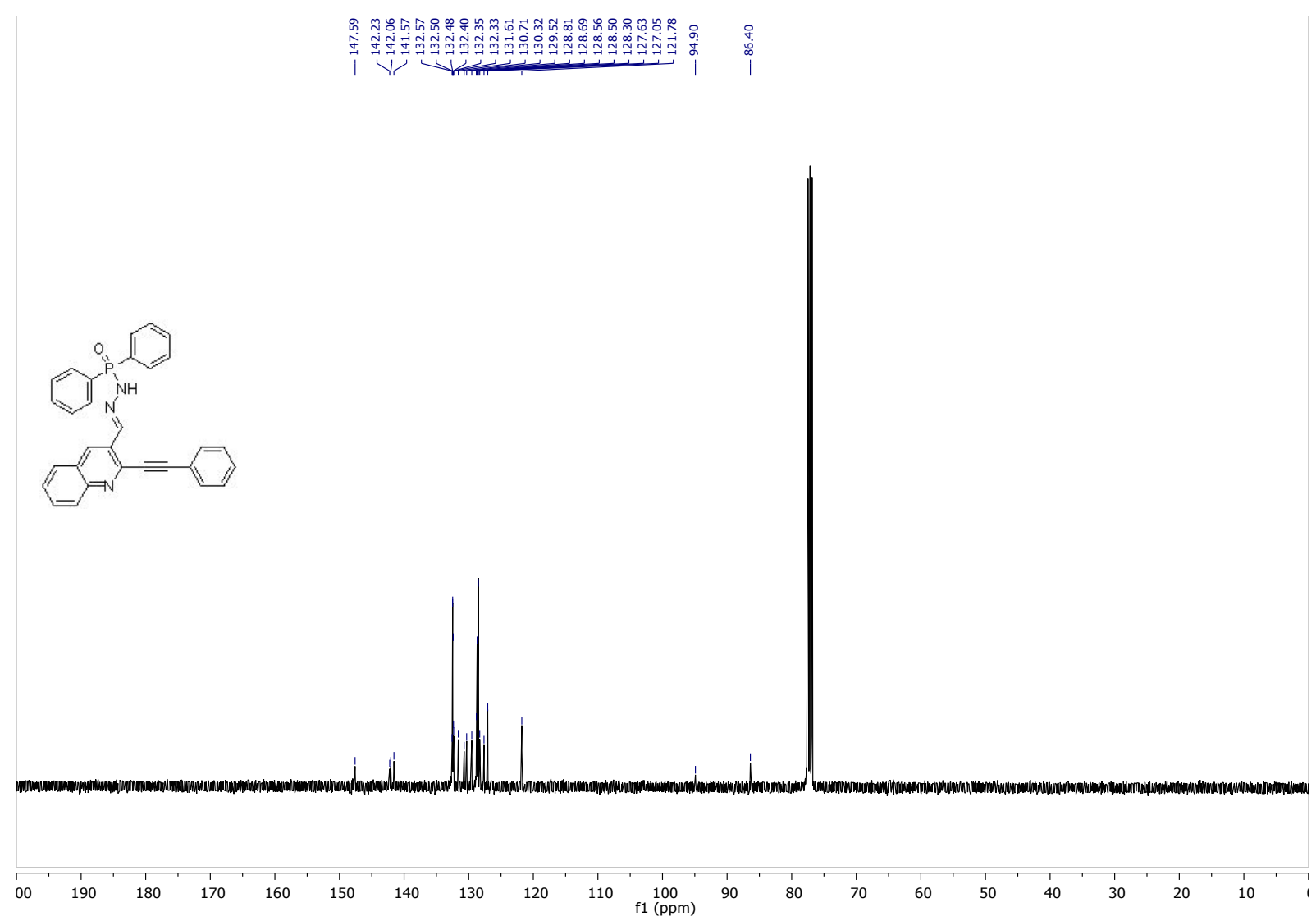


31P NMR (162 MHz, Chloroform-d)

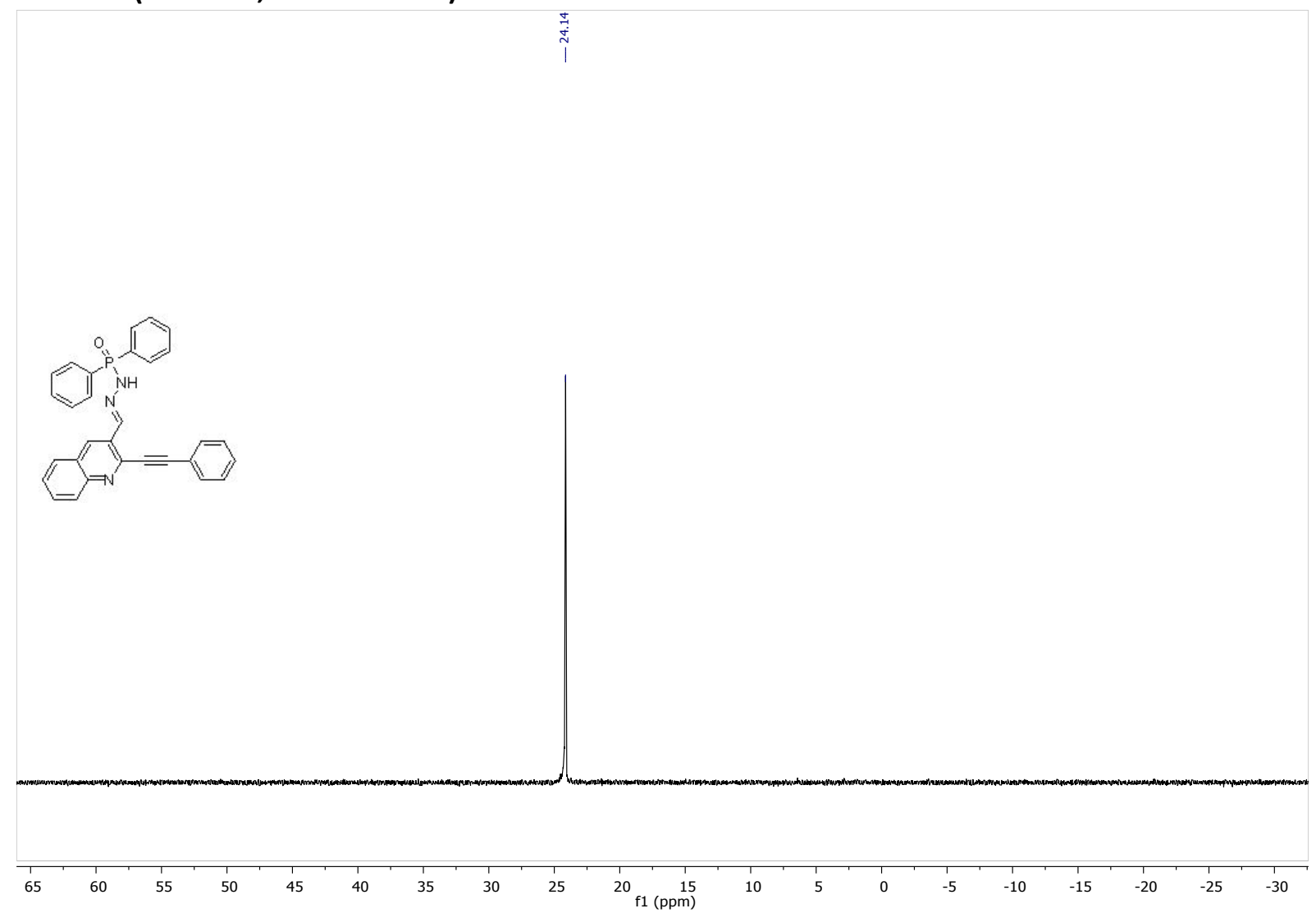


Compound 1p: N'-(2-(phenylethynyl)benzylidene)-P,P-di-p-tolylphosphinic hydrazide ${ }^{1} \mathrm{H}$ NMR (400 MHz, Chloroform-d)

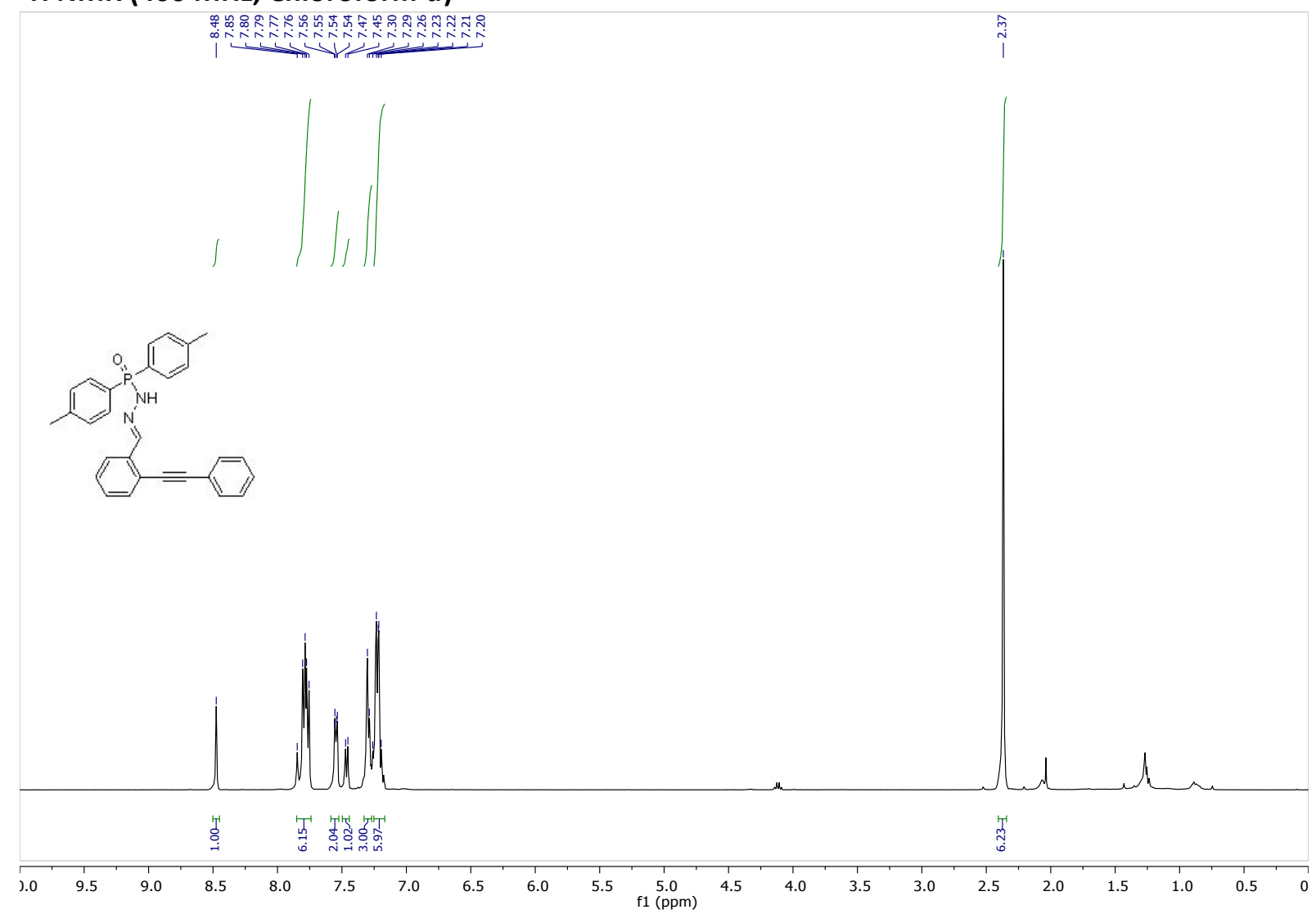

${ }^{13} \mathrm{C}\left\{{ }^{1} \mathrm{H}\right\}$ NMR (101 MHz, Chloroform-d)

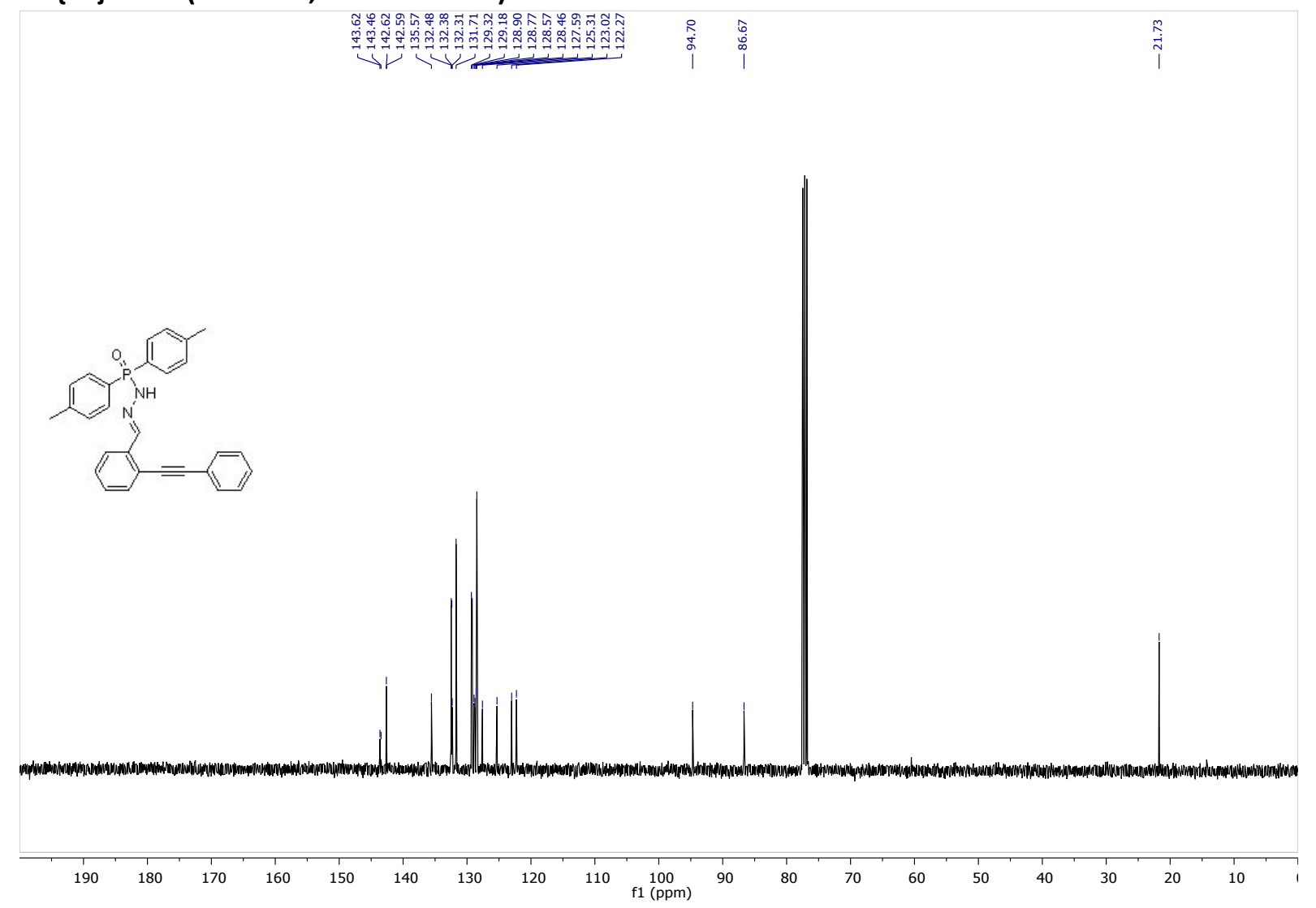

S40 
31P NMR (162 MHz, Chloroform-d)
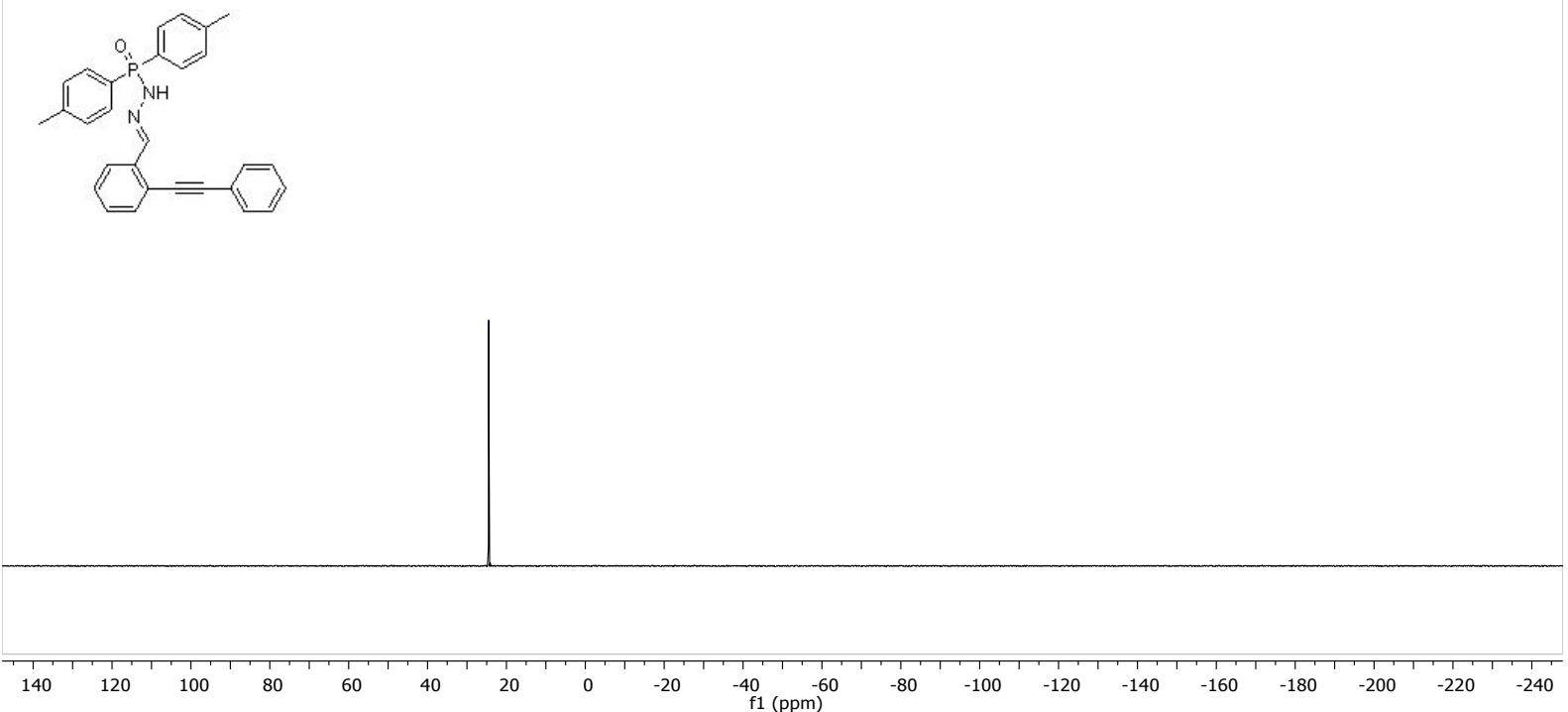
Compound 1q: $\mathrm{N}^{\prime}$-(2-(phenylethynyl)benzylidene)-P,P-di-o-tolylphosphinic hydrazide ${ }^{1} \mathrm{H}$ NMR (400 MHz, Chloroform-d)

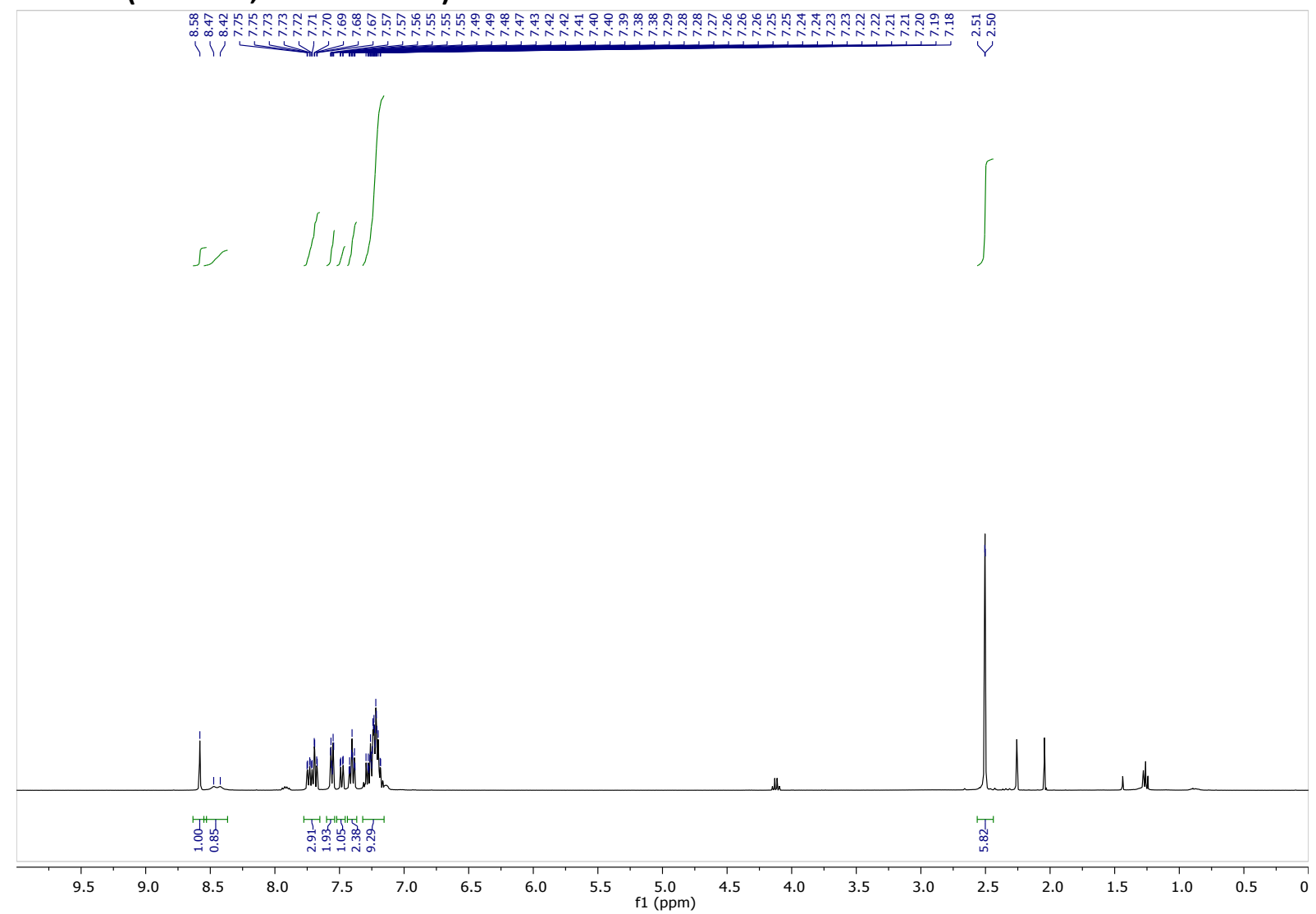

${ }^{13} \mathrm{C}\left\{{ }^{1} \mathrm{H}\right\}$ NMR (101 MHz, Chloroform-d) (JMOD) spectrum

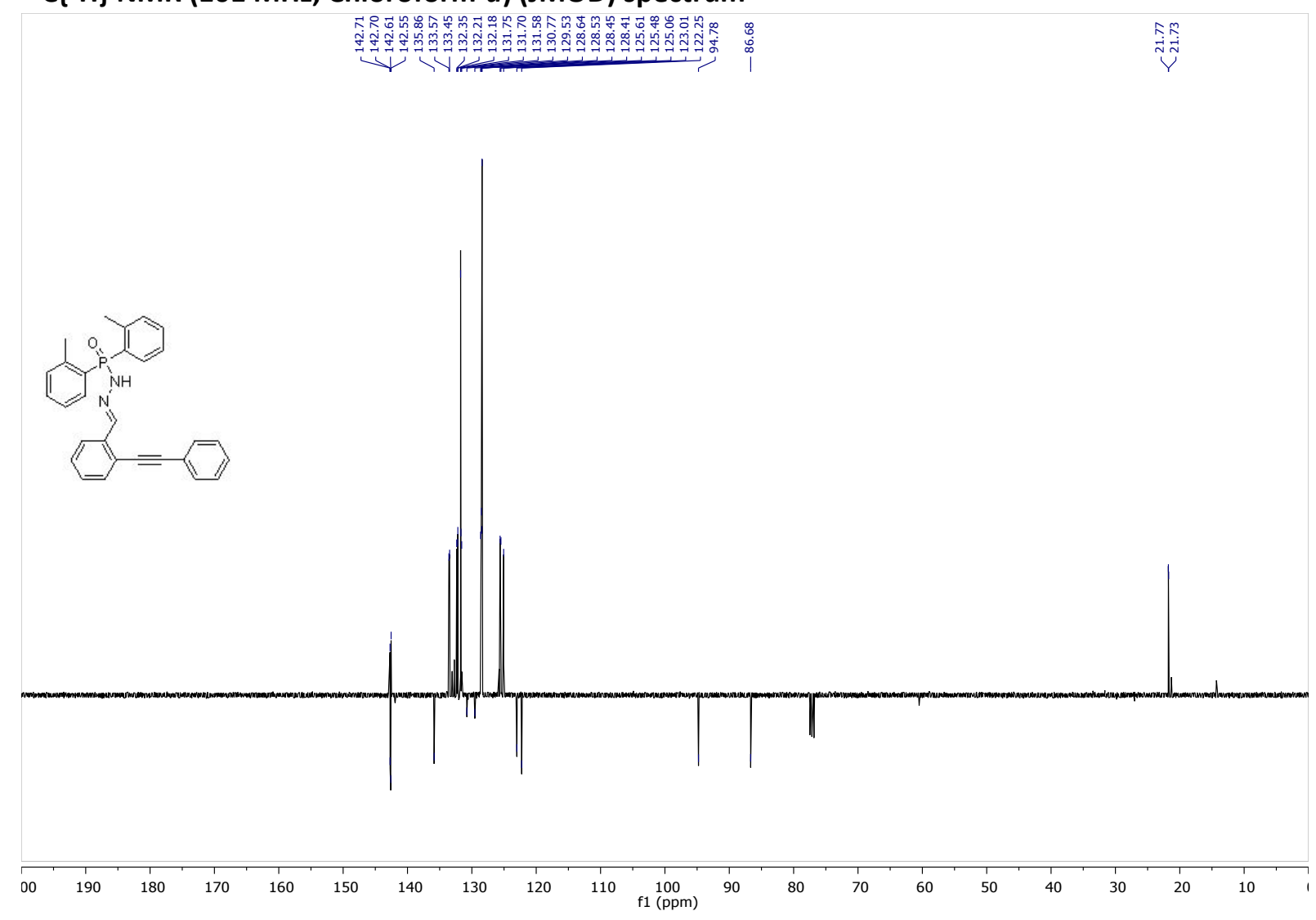


31P NMR (121 MHz, Chloroform-d)

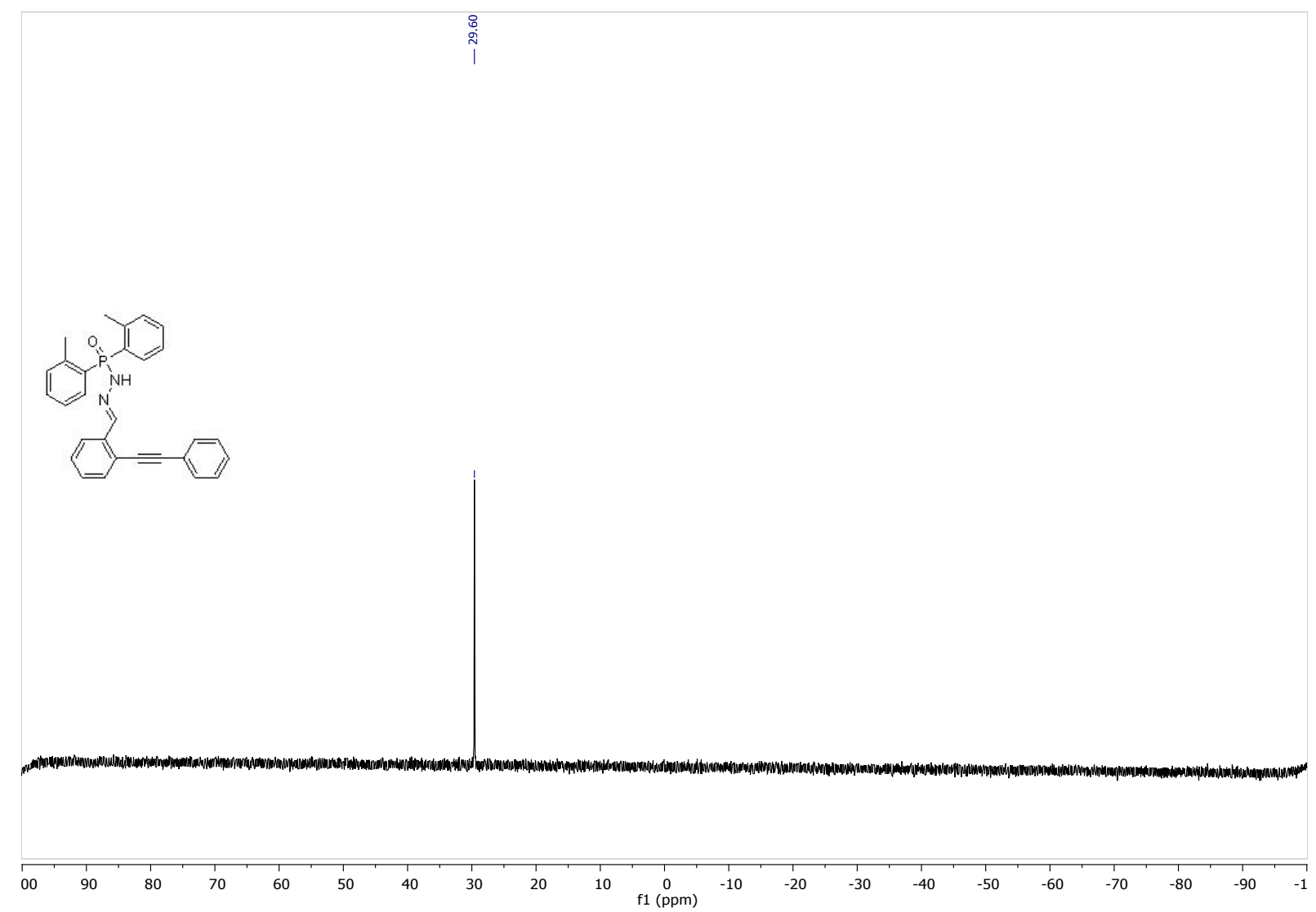

S43 
Compound 1r: N'-(2-(phenylethynyl)benzylidene)-P,P-bis(3,4,5-trimethoxyphenyl)phosphinic hydrazide

${ }^{1} \mathrm{H}$ NMR (300 MHz, Chloroform-d)

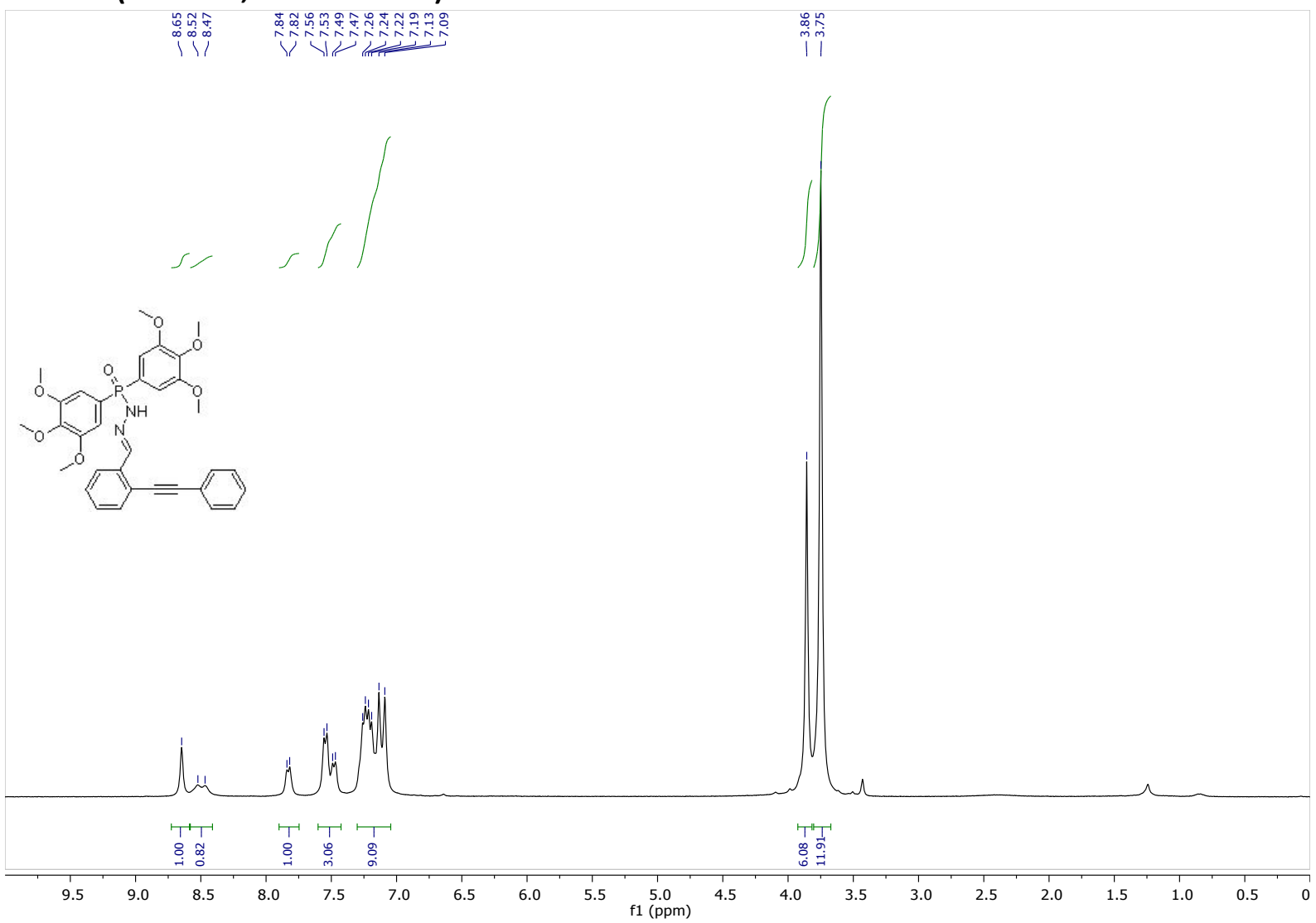

${ }^{13} \mathrm{C}\left\{{ }^{1} \mathrm{H}\right\}$ NMR (75 MHz, Chloroform-d) (JMOD) spectrum

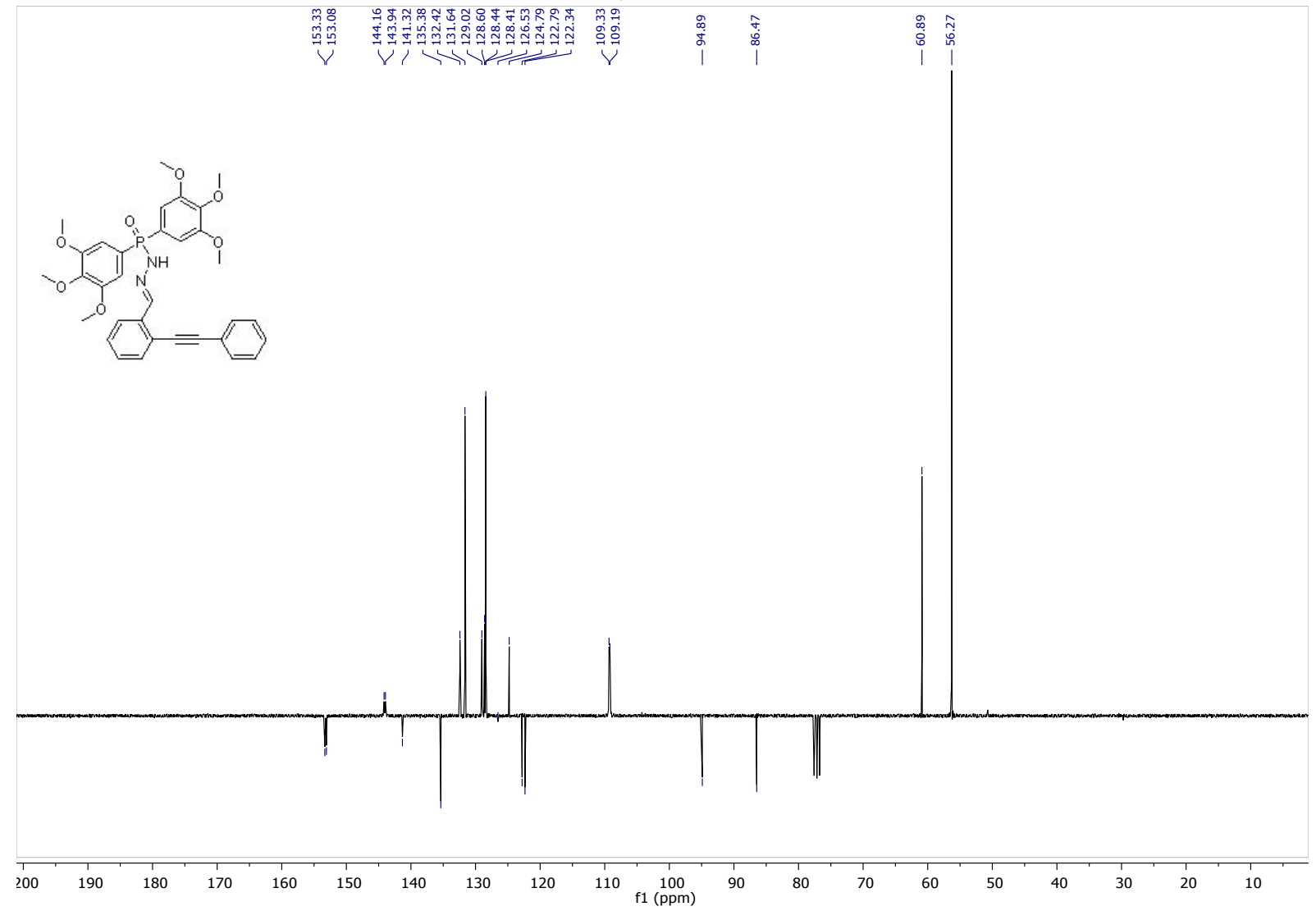


31P NMR (121 MHz, Chloroform-d)

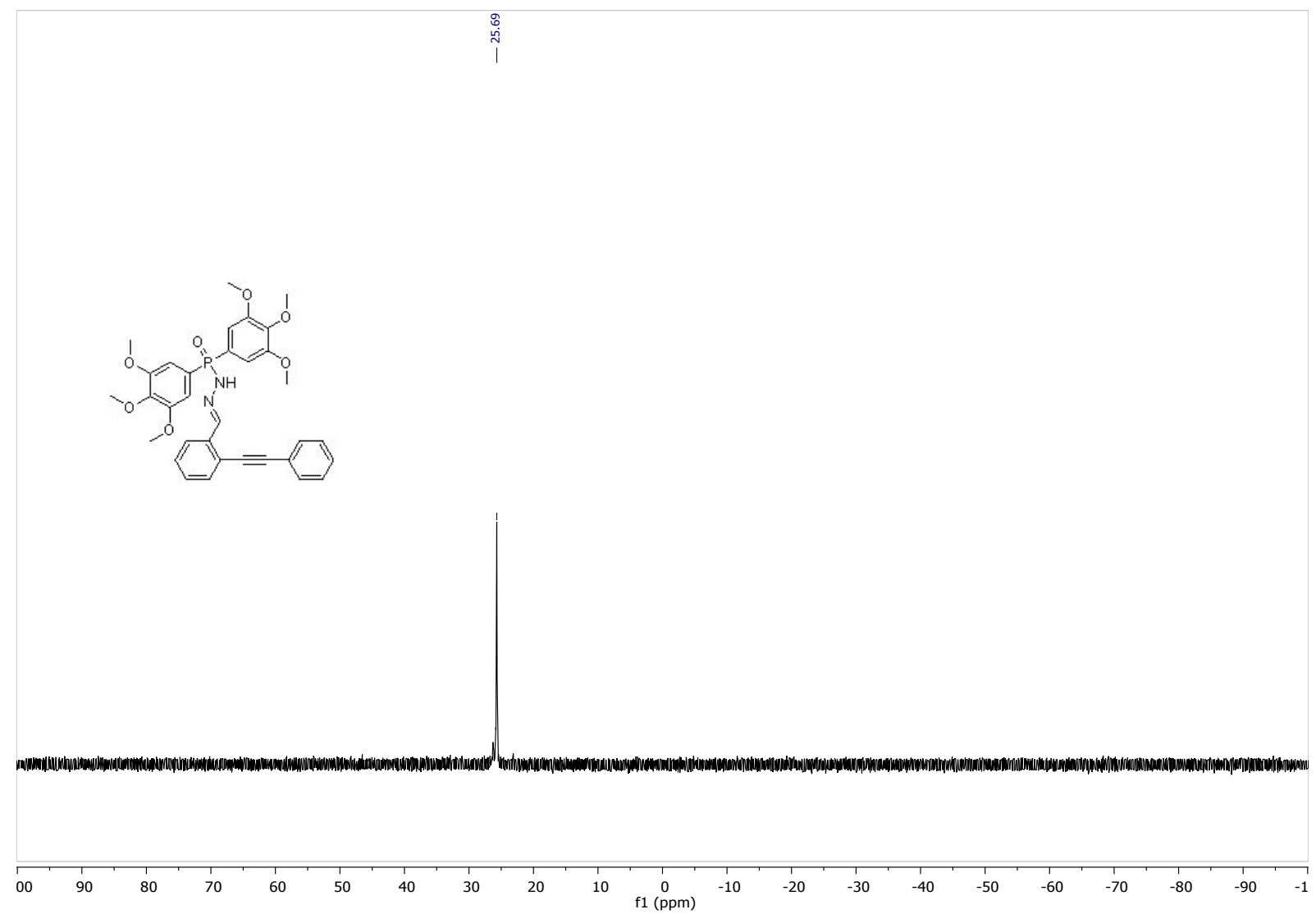


Compound 1s: P,P-bis(benzo[d][1,3]dioxol-5-yl)-N'-(2-(phenylethynyl)benzylidene)phosphinic hydrazide

${ }^{1} \mathrm{H}$ NMR (300 MHz, Chloroform-d)

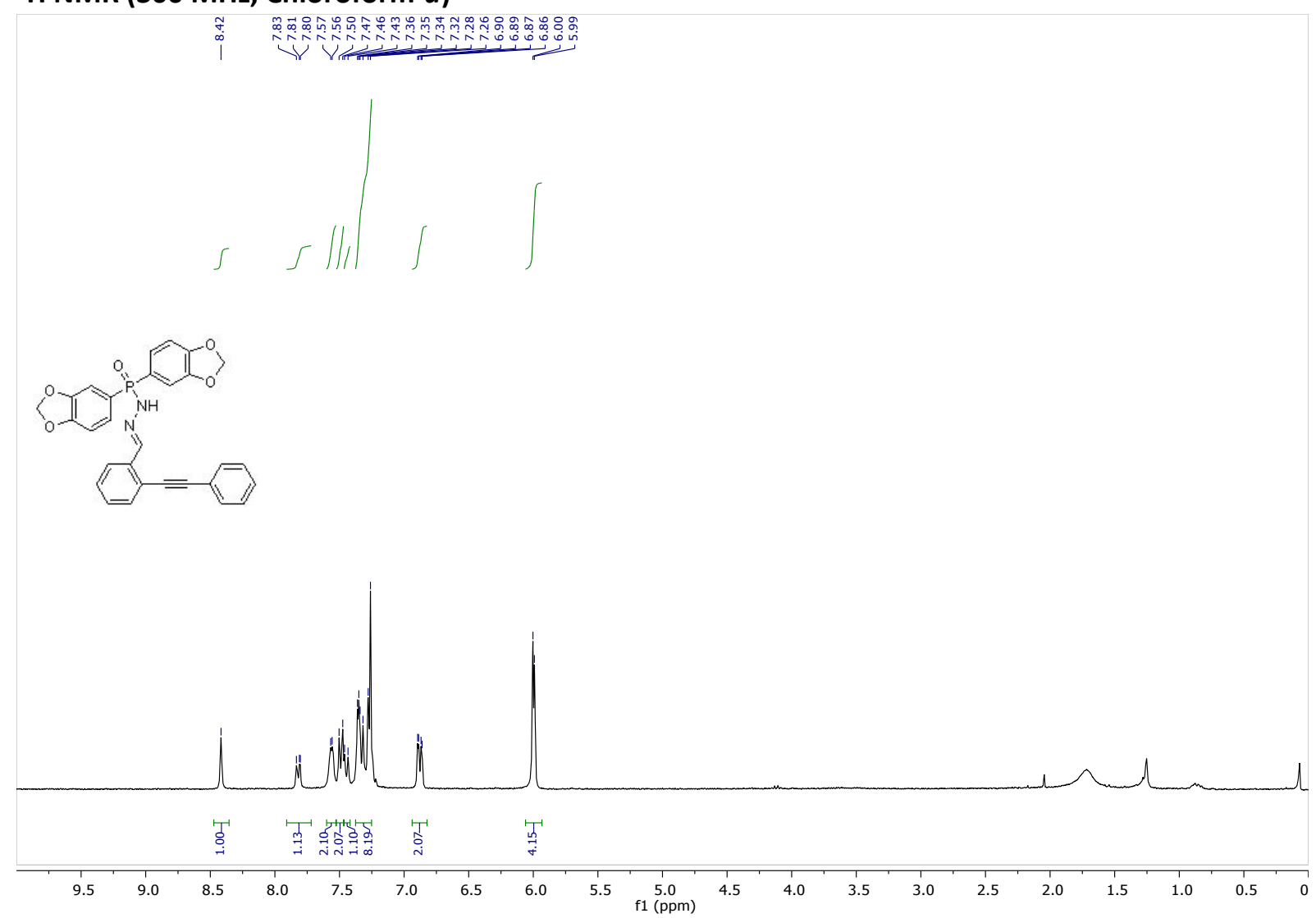

${ }^{13} \mathrm{C}\left\{{ }^{1} \mathrm{H}\right\}$ NMR (75 MHz, Chloroform-d)

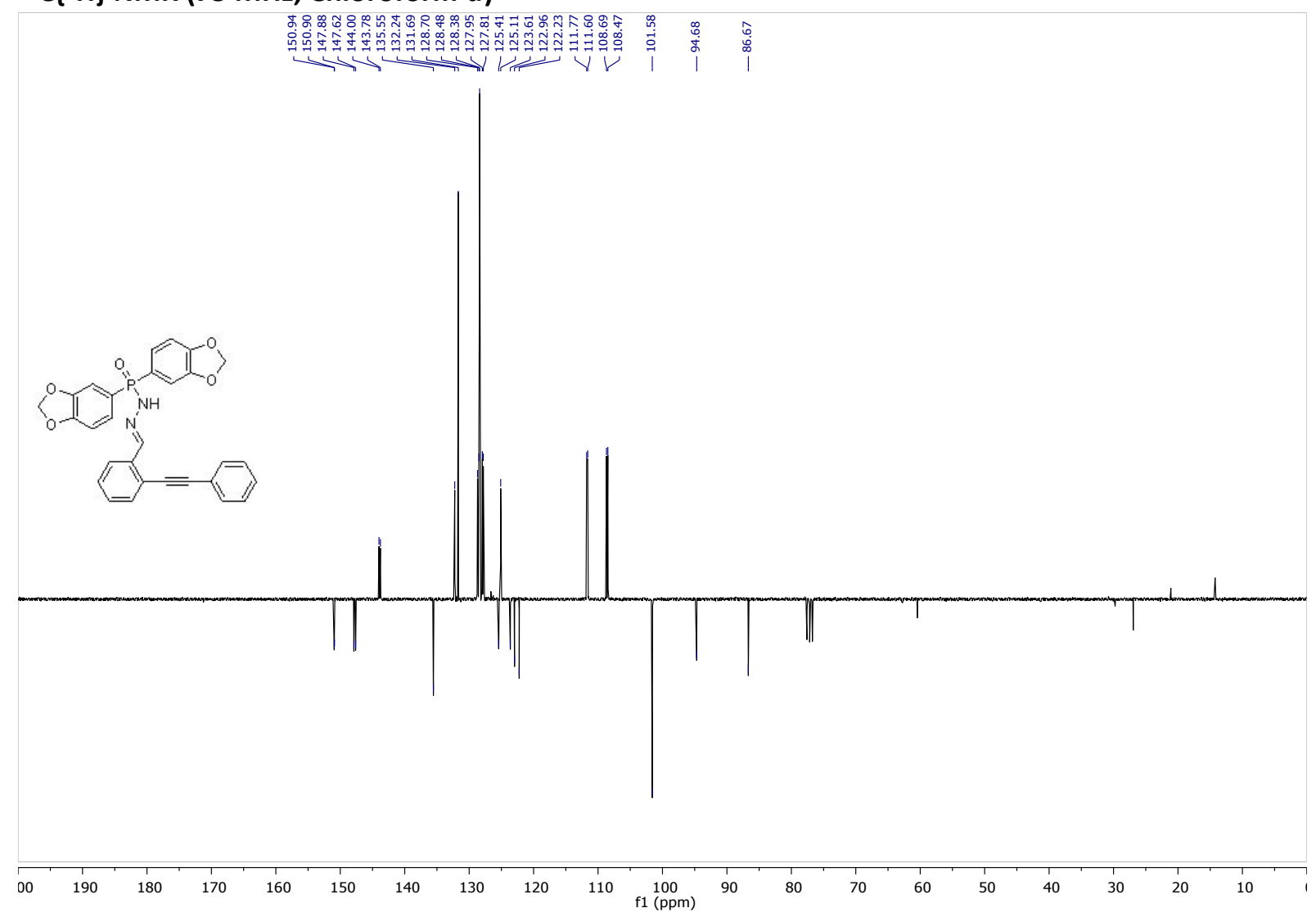


31P NMR (121 MHz, Chloroform-d)
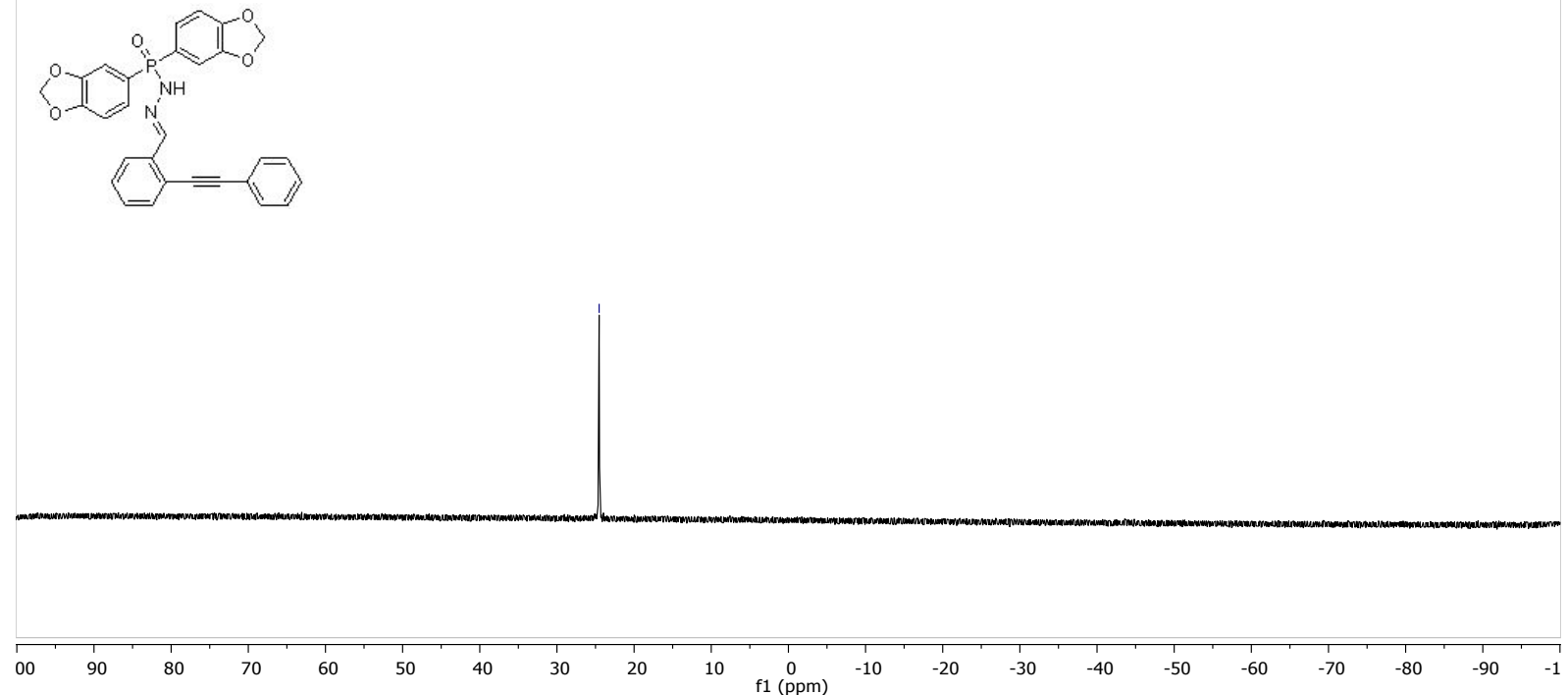
Compound 1t: P,P-bis(4-fluorophenyl)-N'-(2-(phenylethynyl)benzylidene)phosphinic hydrazide ${ }^{1} \mathrm{H}$ NMR (400 MHz, Chloroform-d)

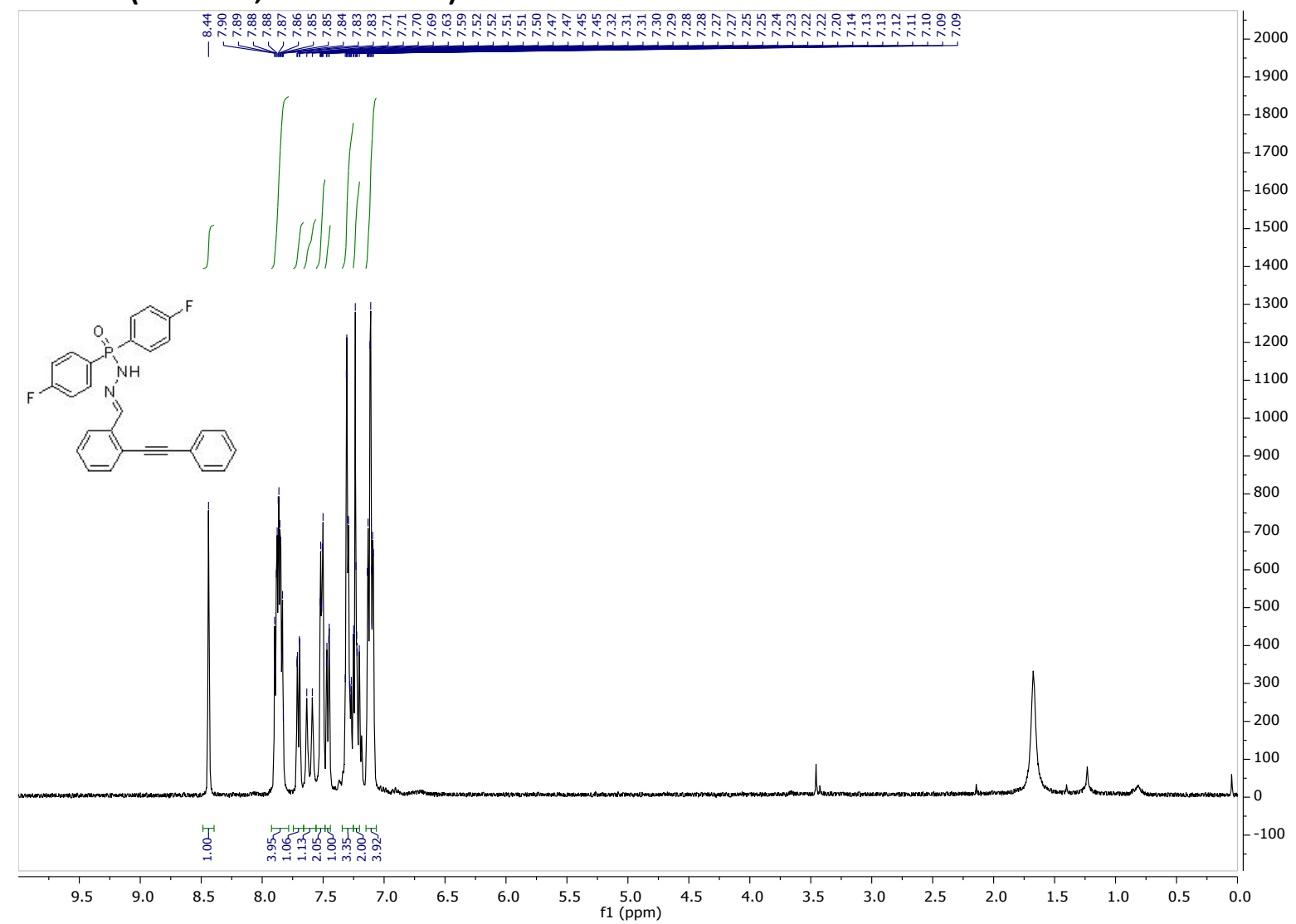

${ }^{13} \mathrm{C}\left\{{ }^{1} \mathrm{H}\right\}$ NMR (101 MHz, Chloroform-d)

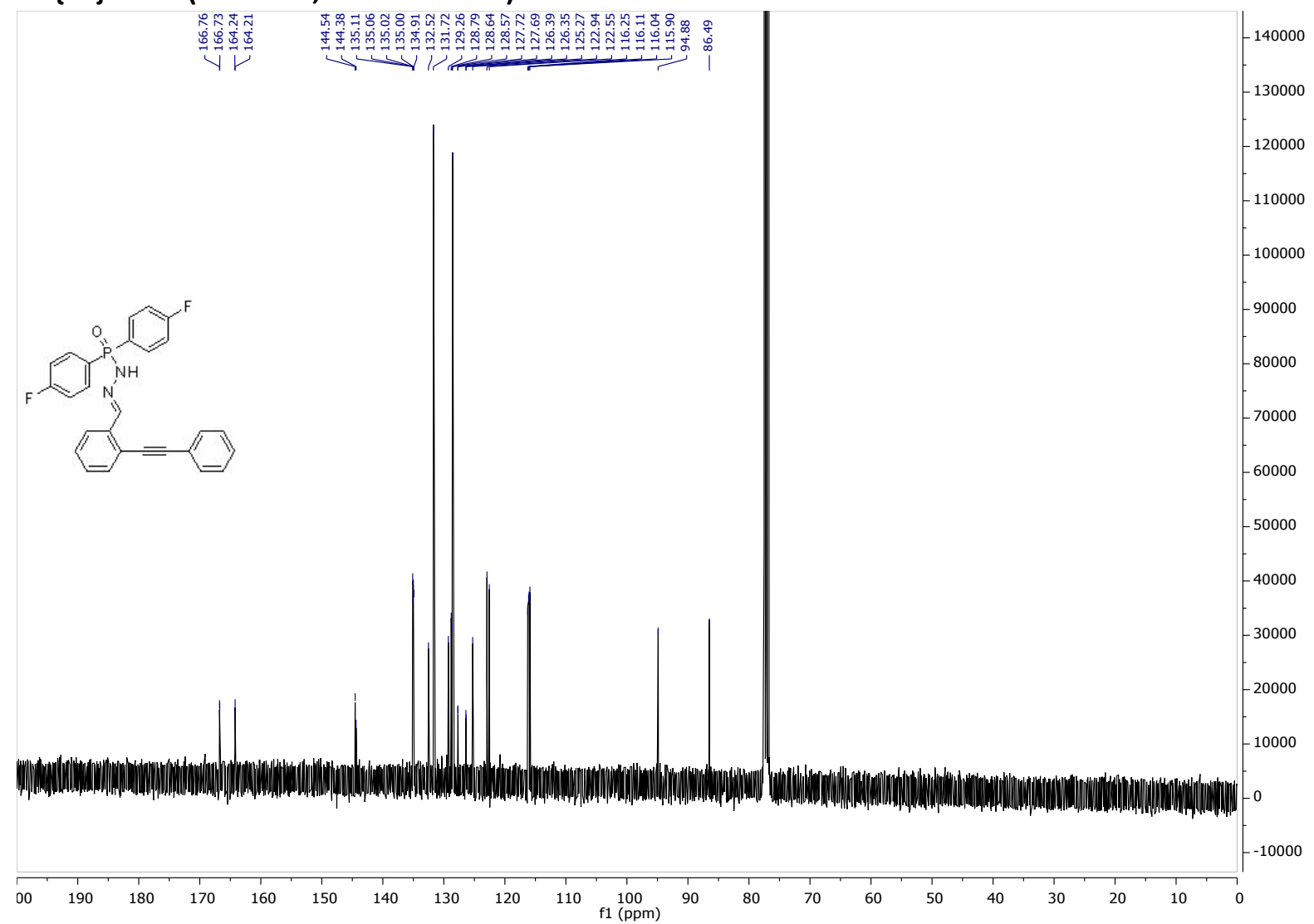


31P NMR (162 MHz, Chloroform-d)

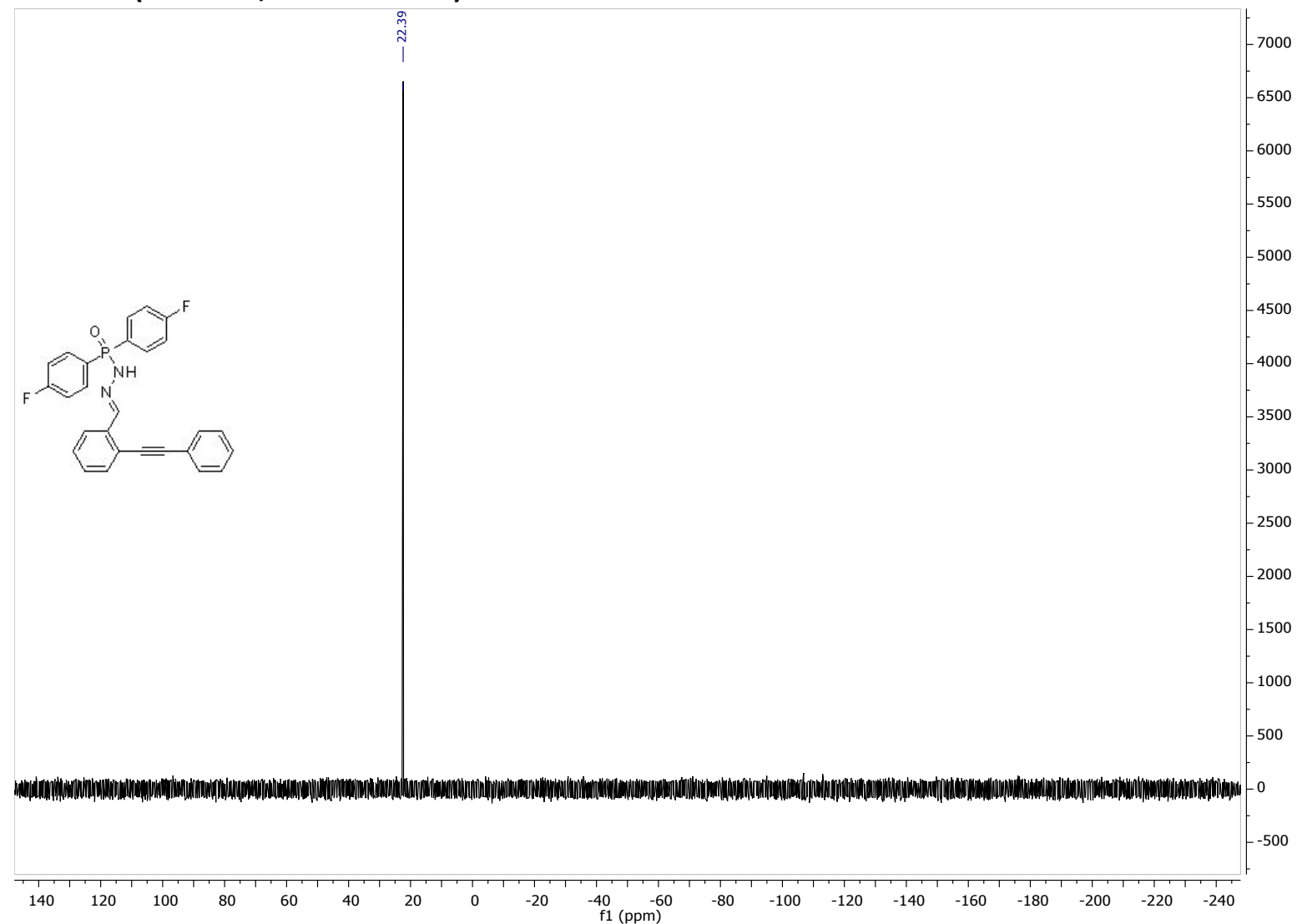

${ }^{19}$ f NMR (376 MHz, Chloroform-d)

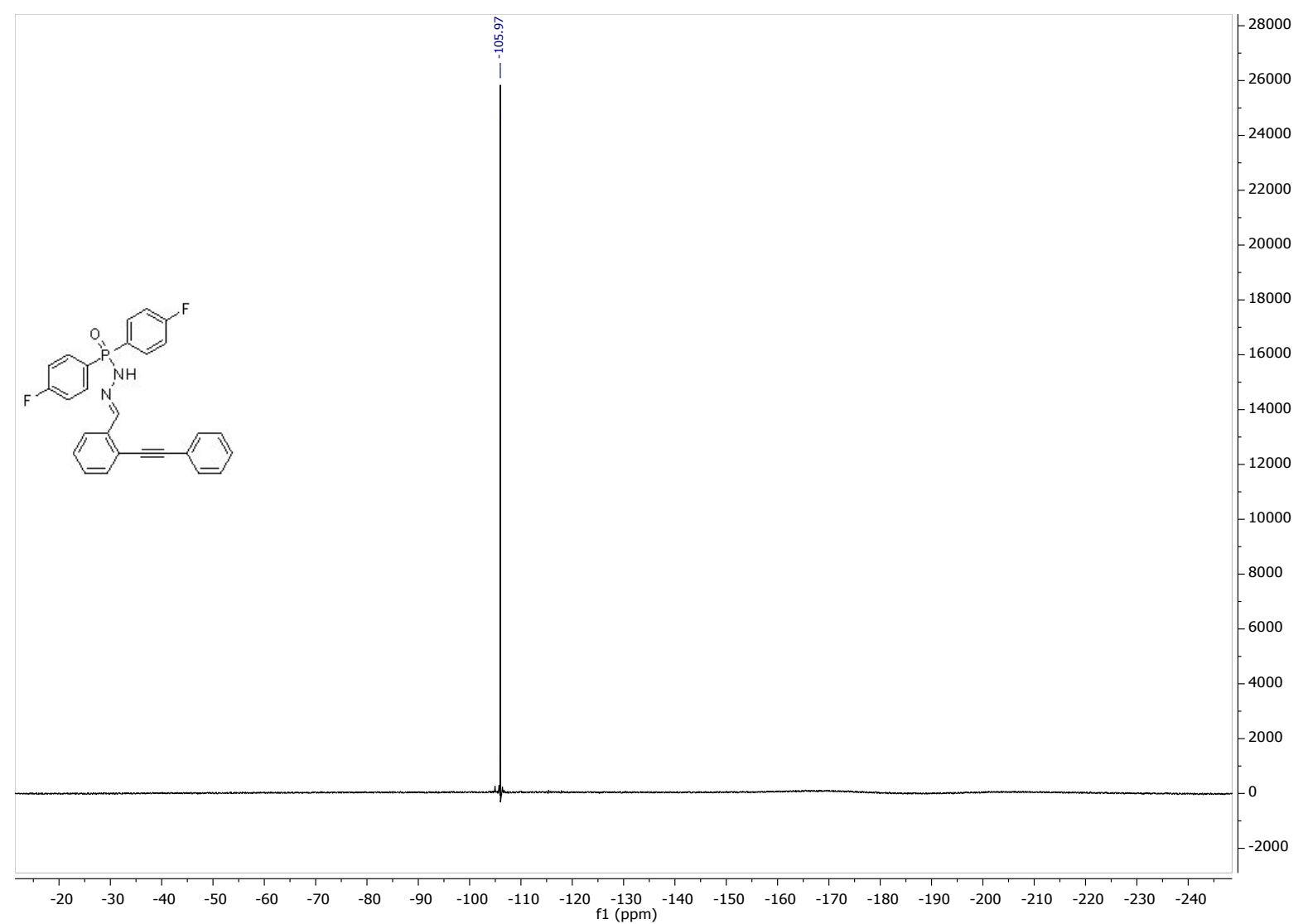


Compound 1u: P,P-bis(4-chlorophenyl)-N'-(2-(phenylethynyl)benzylidene)phosphinic hydrazide ${ }^{1} \mathrm{H}$ NMR (400 MHz, Chloroform-d)

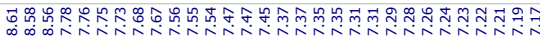
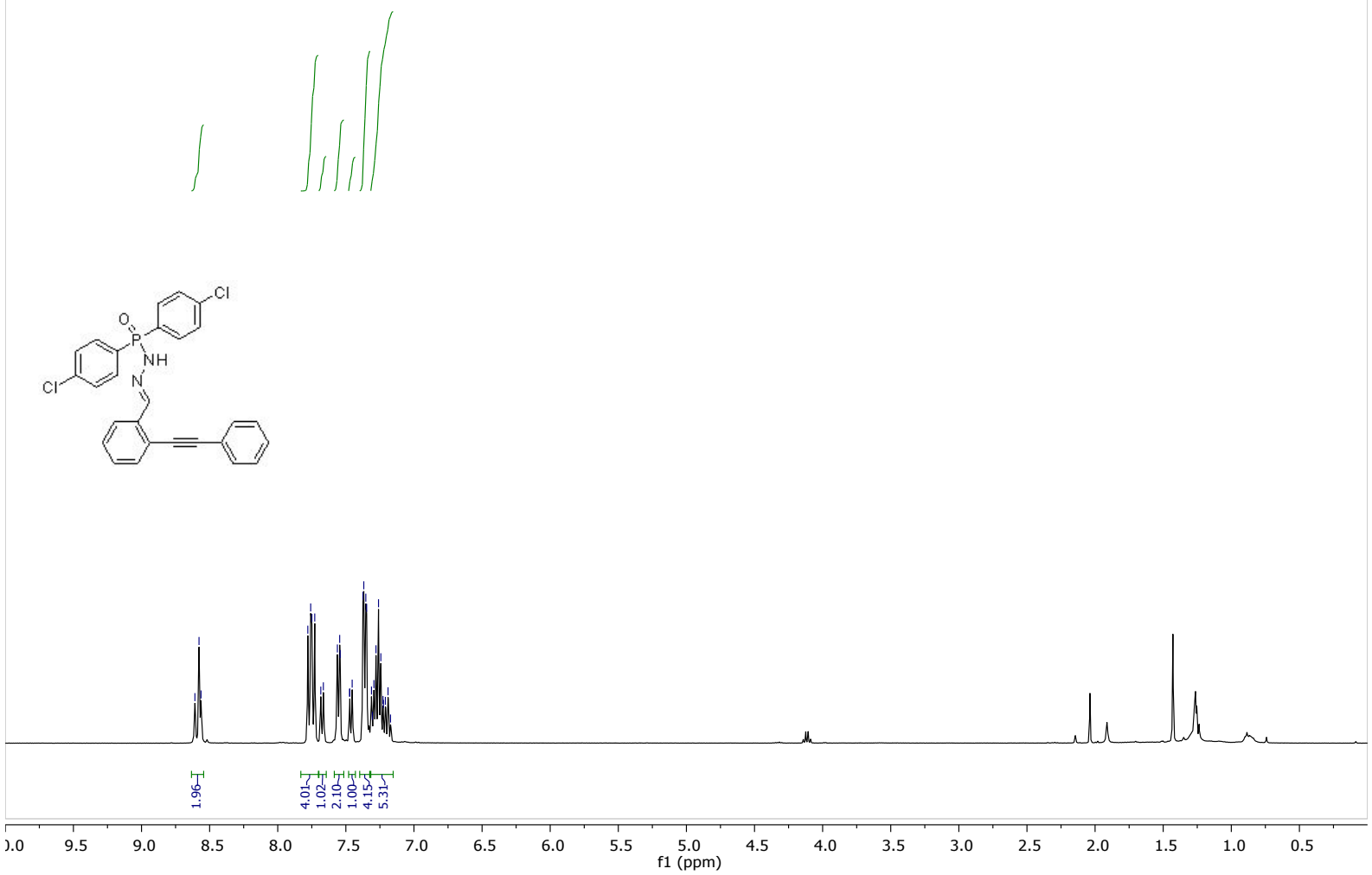

${ }^{13} \mathrm{C}\left\{{ }^{1} \mathrm{H}\right\}$ NMR (101 MHz, Chloroform-d)

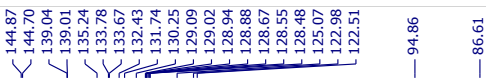
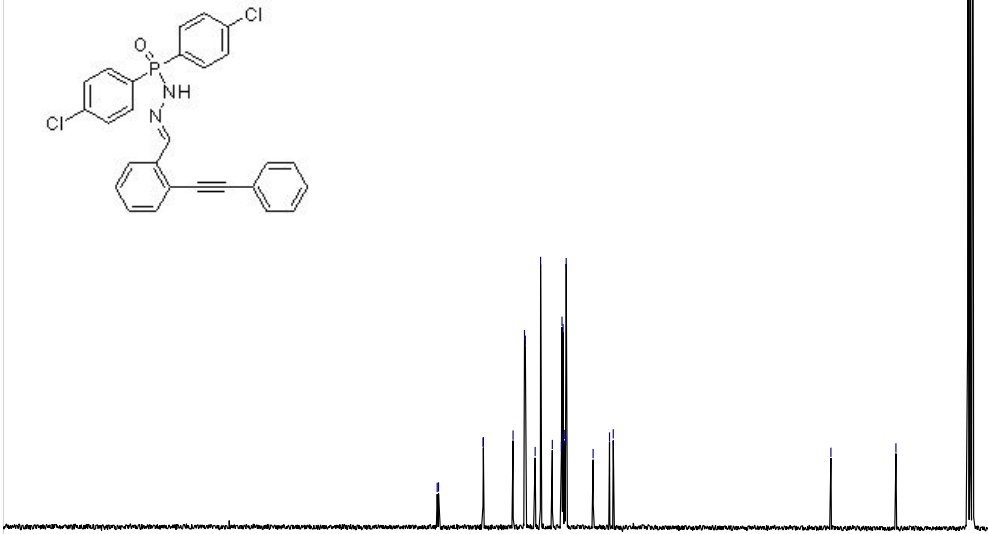

190

$\begin{array}{lll}180 & 170 \quad 160\end{array}$

150

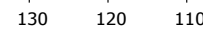

100
$\mathrm{f} 1(\mathrm{ppm})$
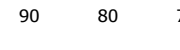
31P NMR (162 MHz, Chloroform-d)
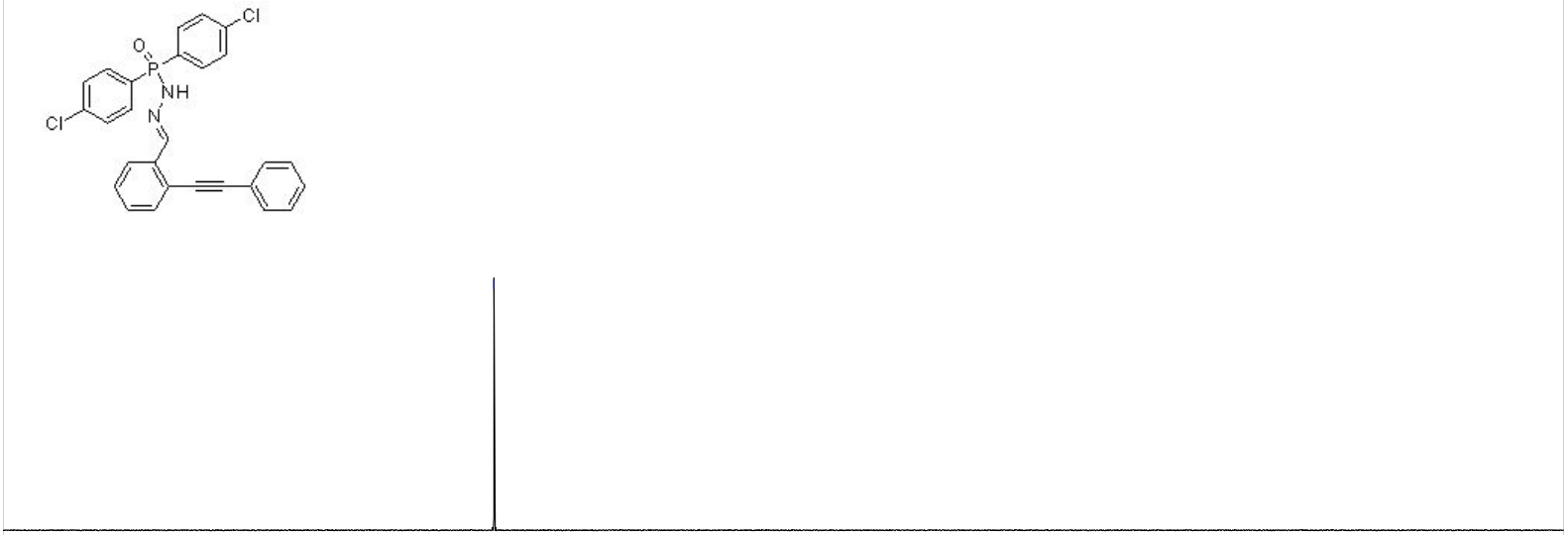

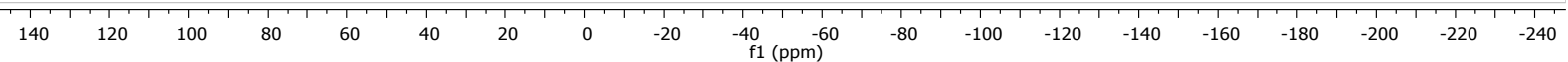


Compound 1v: ethyl (E)-phenyl(2-(2-(phenylethynyl)benzylidene)hydrazineyl)phosphinate ${ }^{1} \mathrm{H}$ NMR (300 MHz, Chloroform-d)

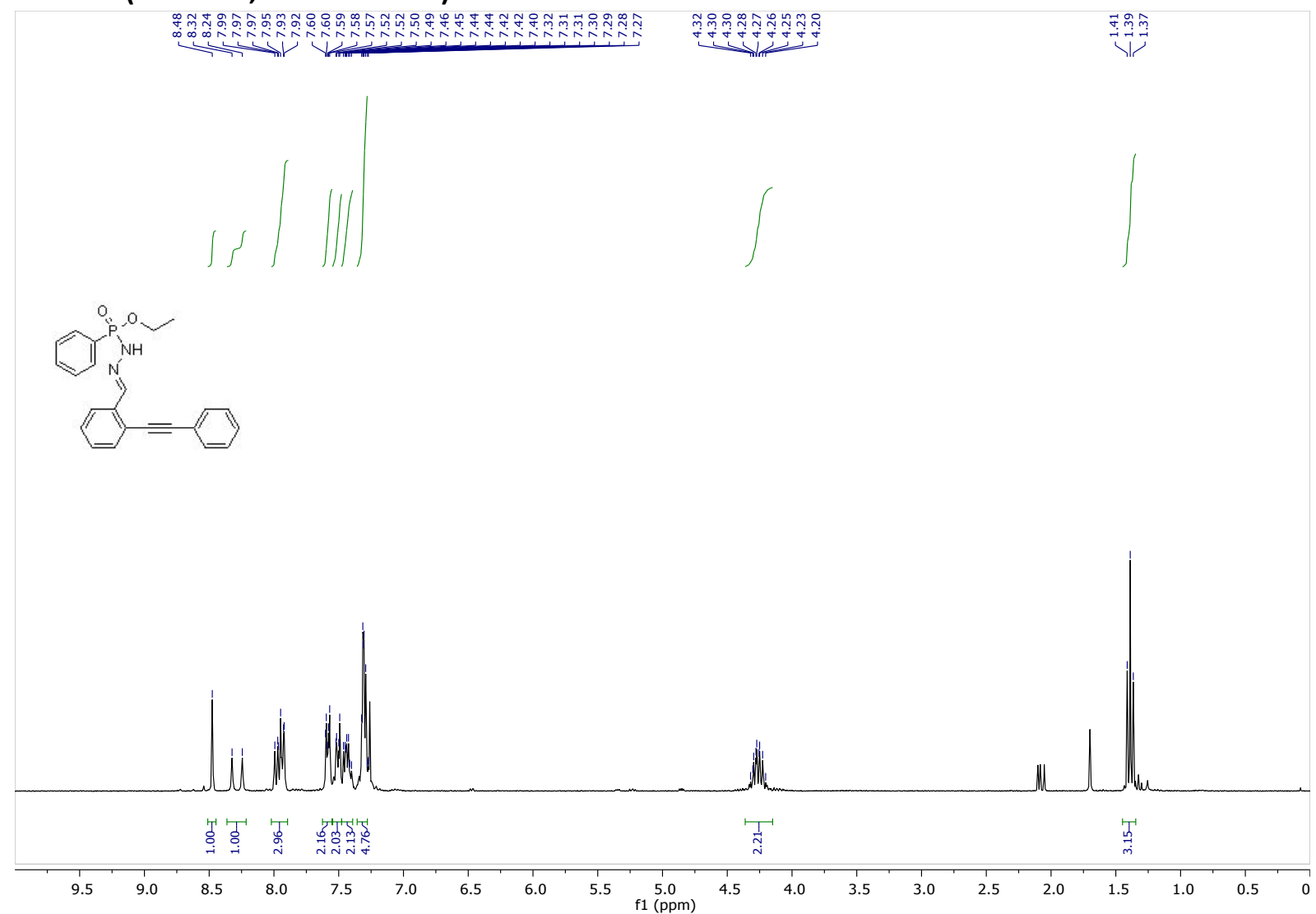

${ }^{13} \mathrm{C}\left\{{ }^{1} \mathrm{H}\right\}$ NMR (75 MHz, Chloroform-d)

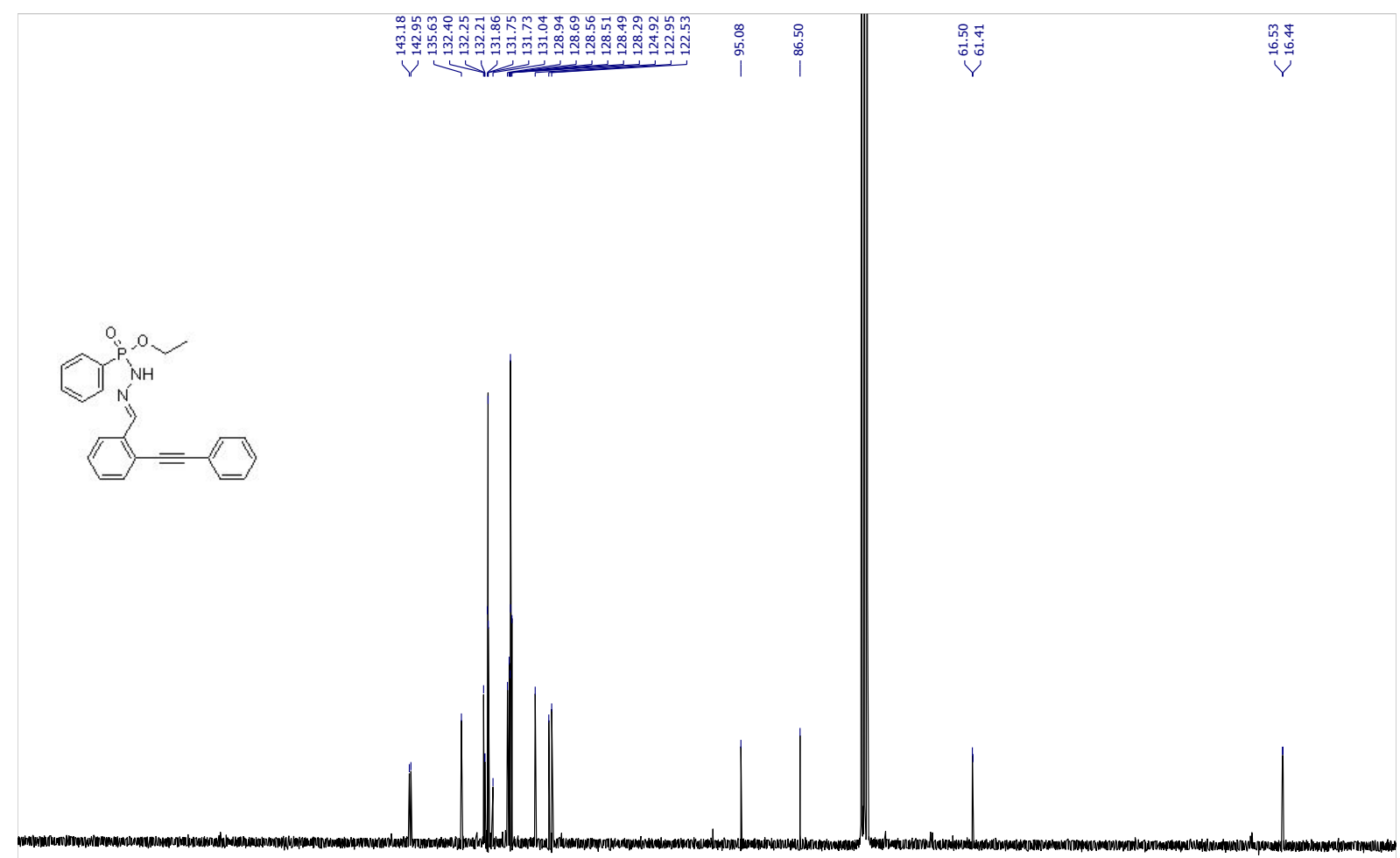


${ }^{31} \mathrm{P}$ NMR (162 MHz, Chloroform-d)

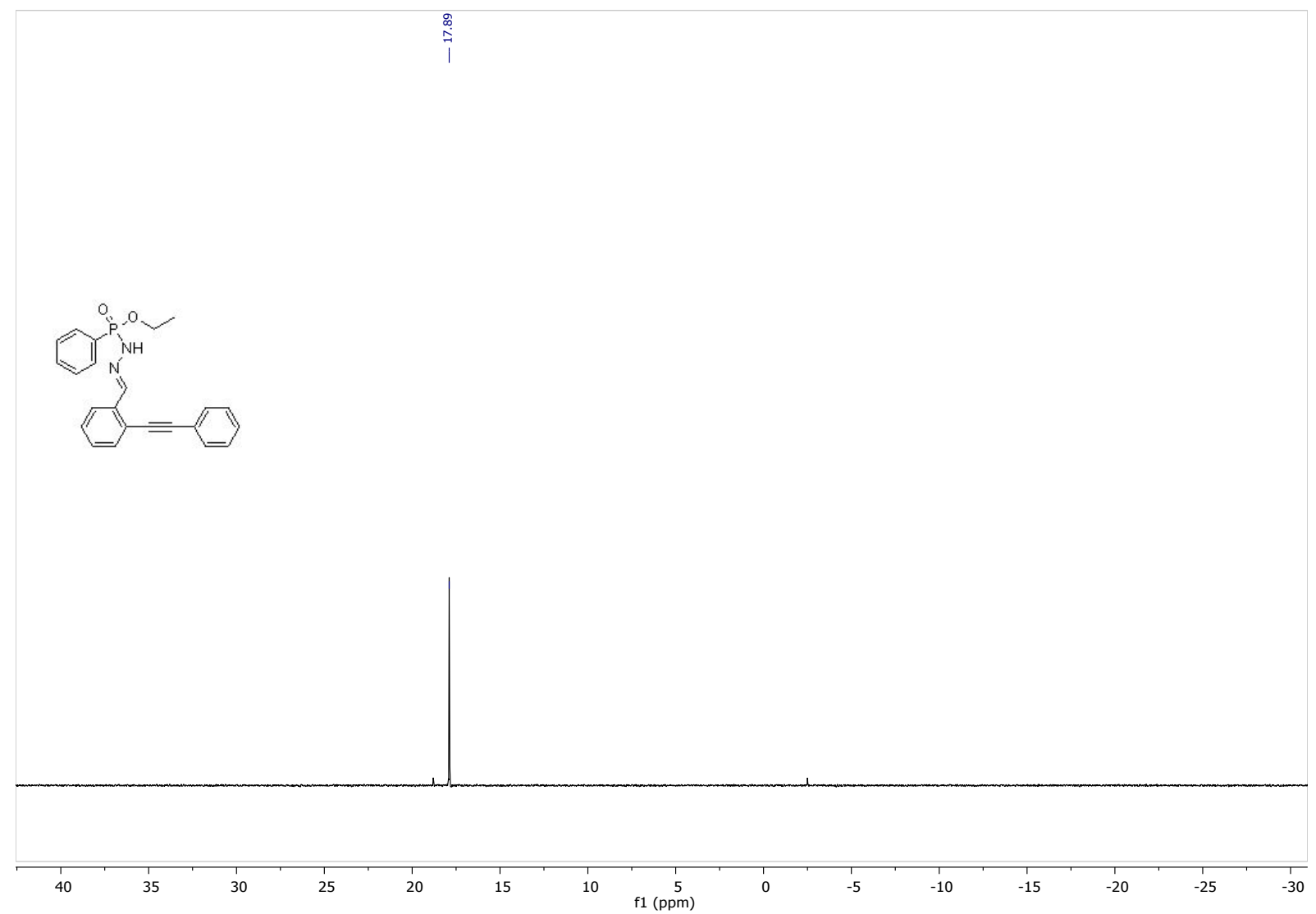


Compound 1W: N'-(2-((4-cyanophenyl)ethynyl)benzylidene)-P,P-diphenylphosphinic hydrazide ${ }^{1} \mathrm{H}$ NMR (300 MHz, Chloroform-d)

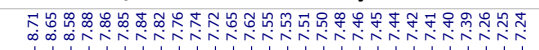

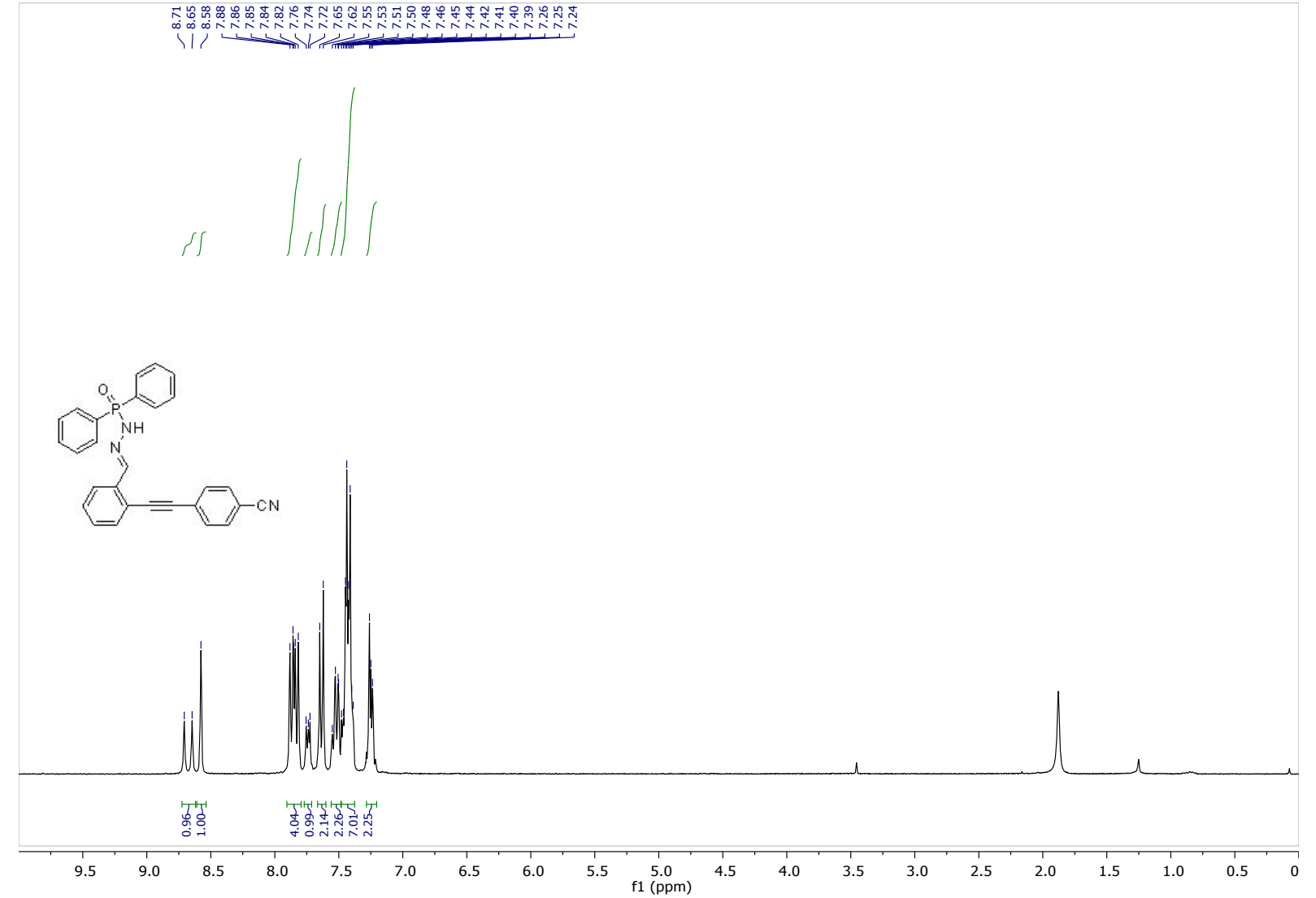

${ }^{13} \mathrm{C}\left\{{ }^{1} \mathrm{H}\right\}$ NMR (75 MHz, Chloroform-d)

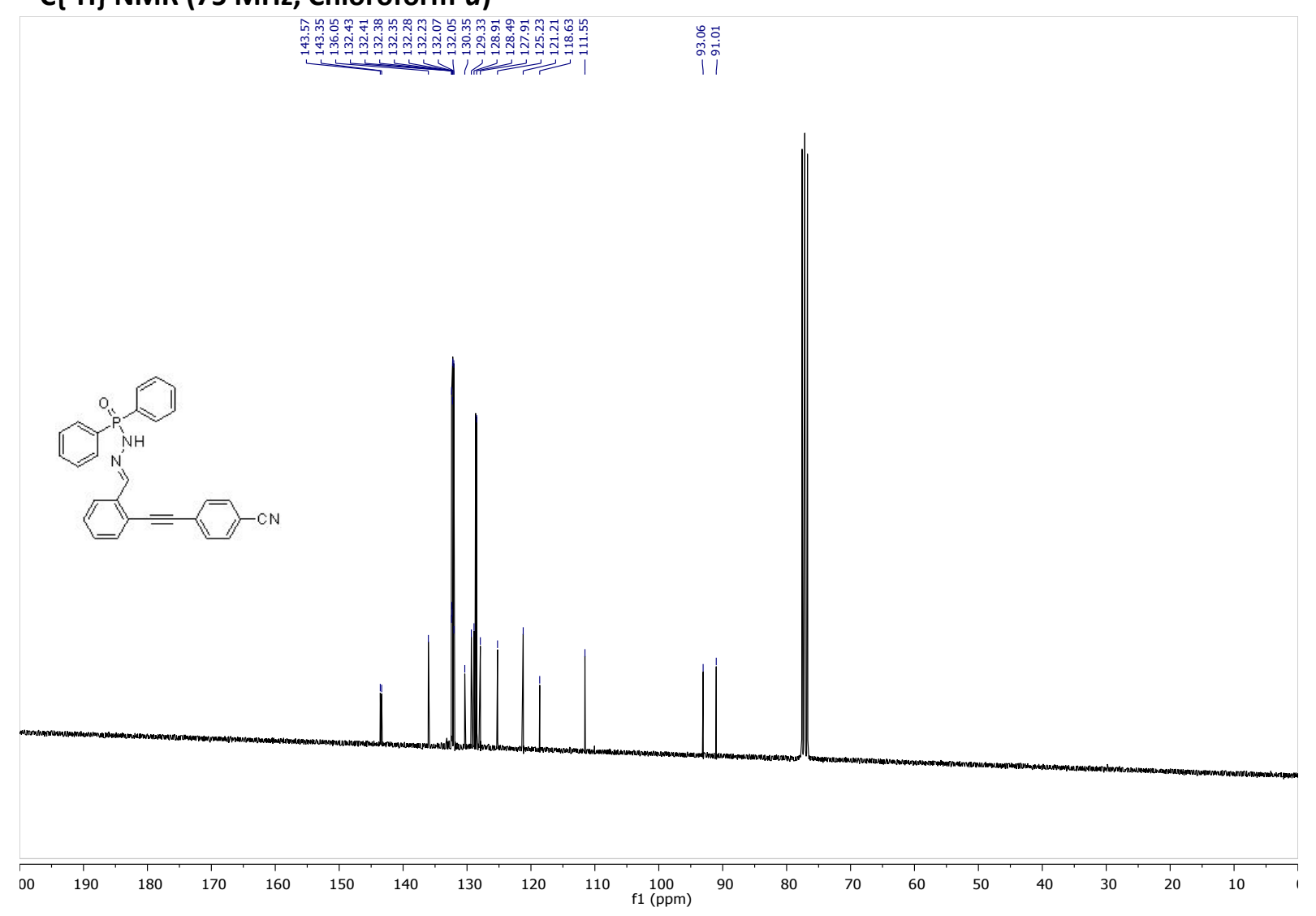

S54 
31P NMR (121 MHz, Chloroform-d)

总

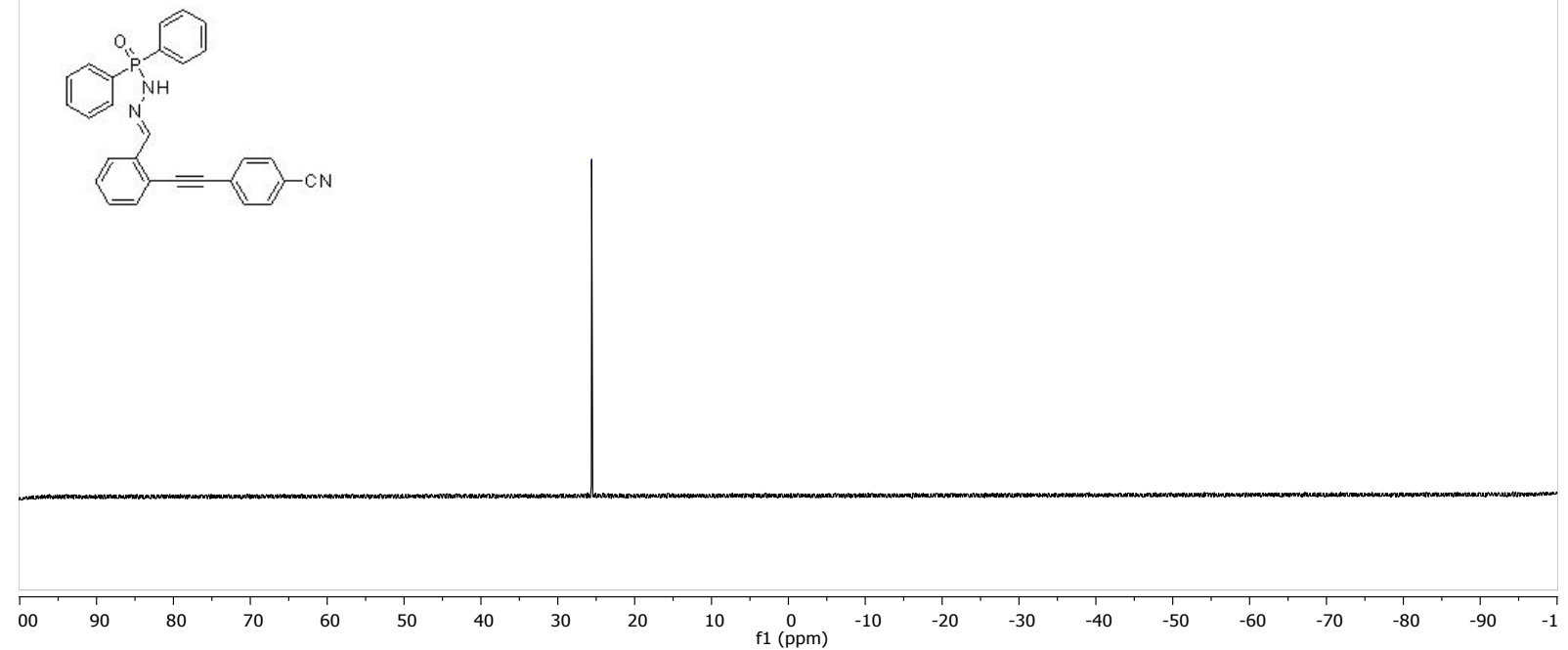


Compound 1x: N'-(2-((3-nitrophenyl)ethynyl)benzylidene)-P,P-diphenylphosphinic hydrazide ${ }^{1} \mathrm{H}$ NMR (300 MHz, Chloroform-d)

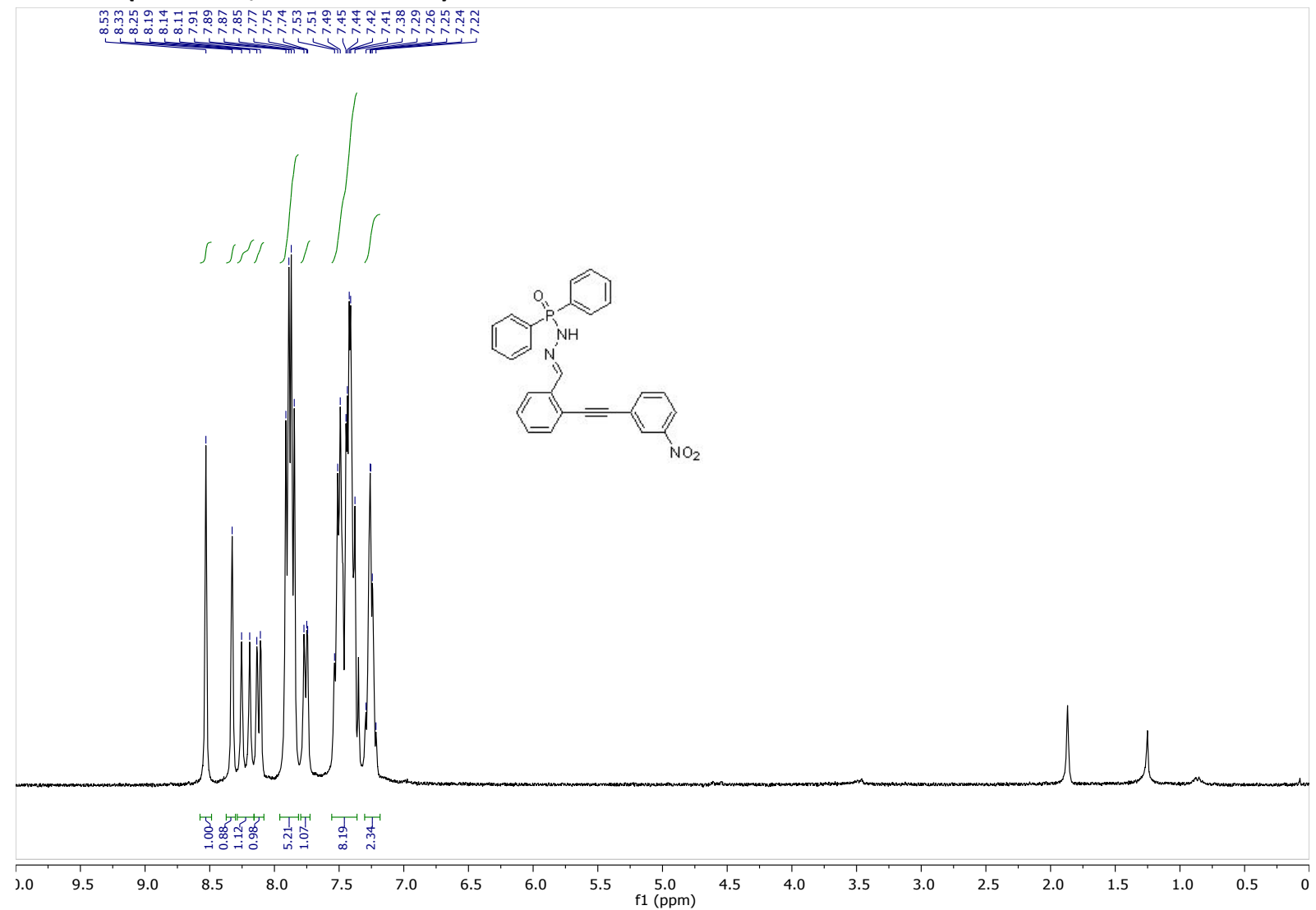

${ }^{13} \mathrm{C}\left\{{ }^{1} \mathrm{H}\right\}$ NMR (75 MHz, Chloroform-d) (JMOD) spectrum

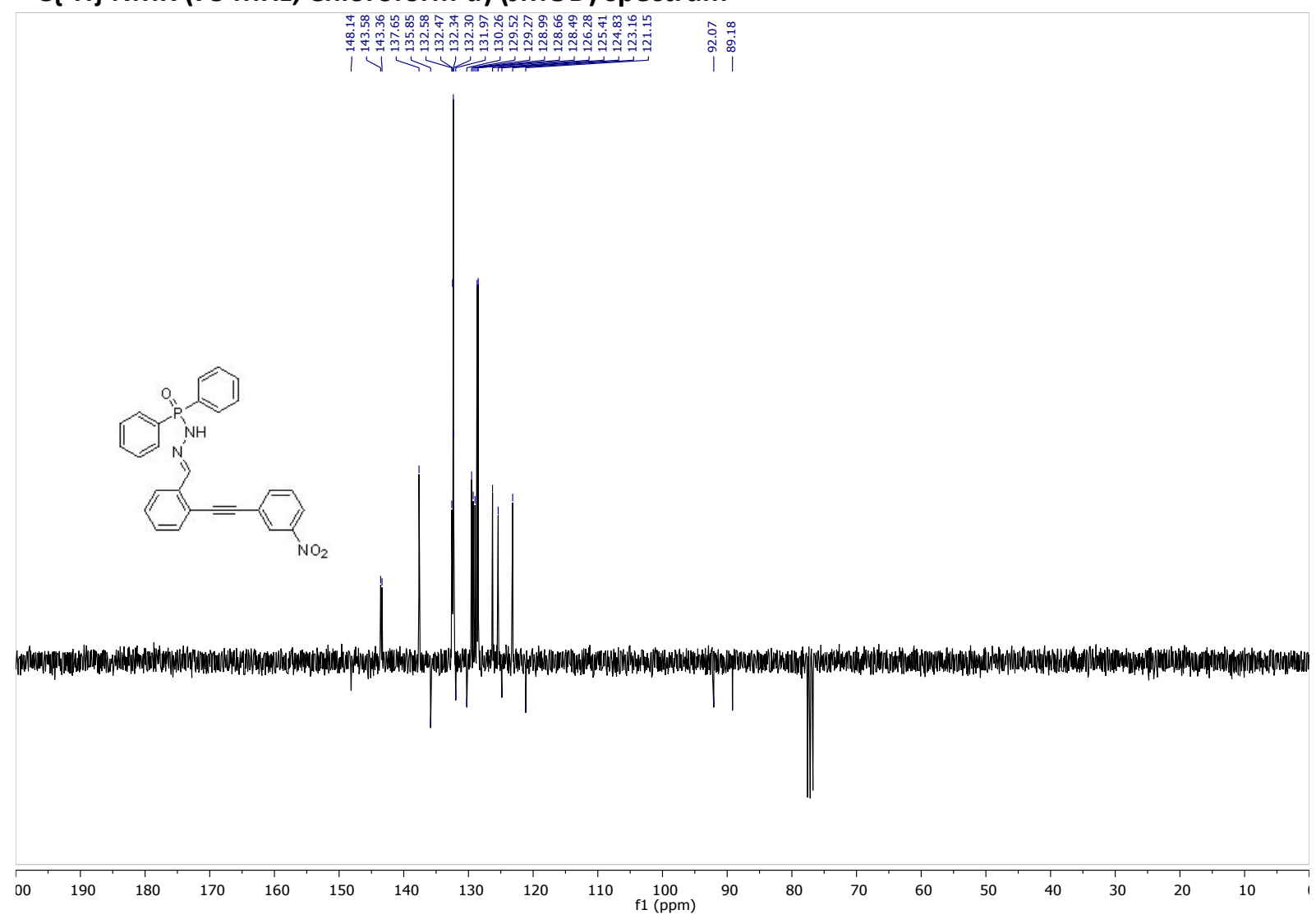


31P NMR (121 MHz, Chloroform-d)

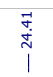

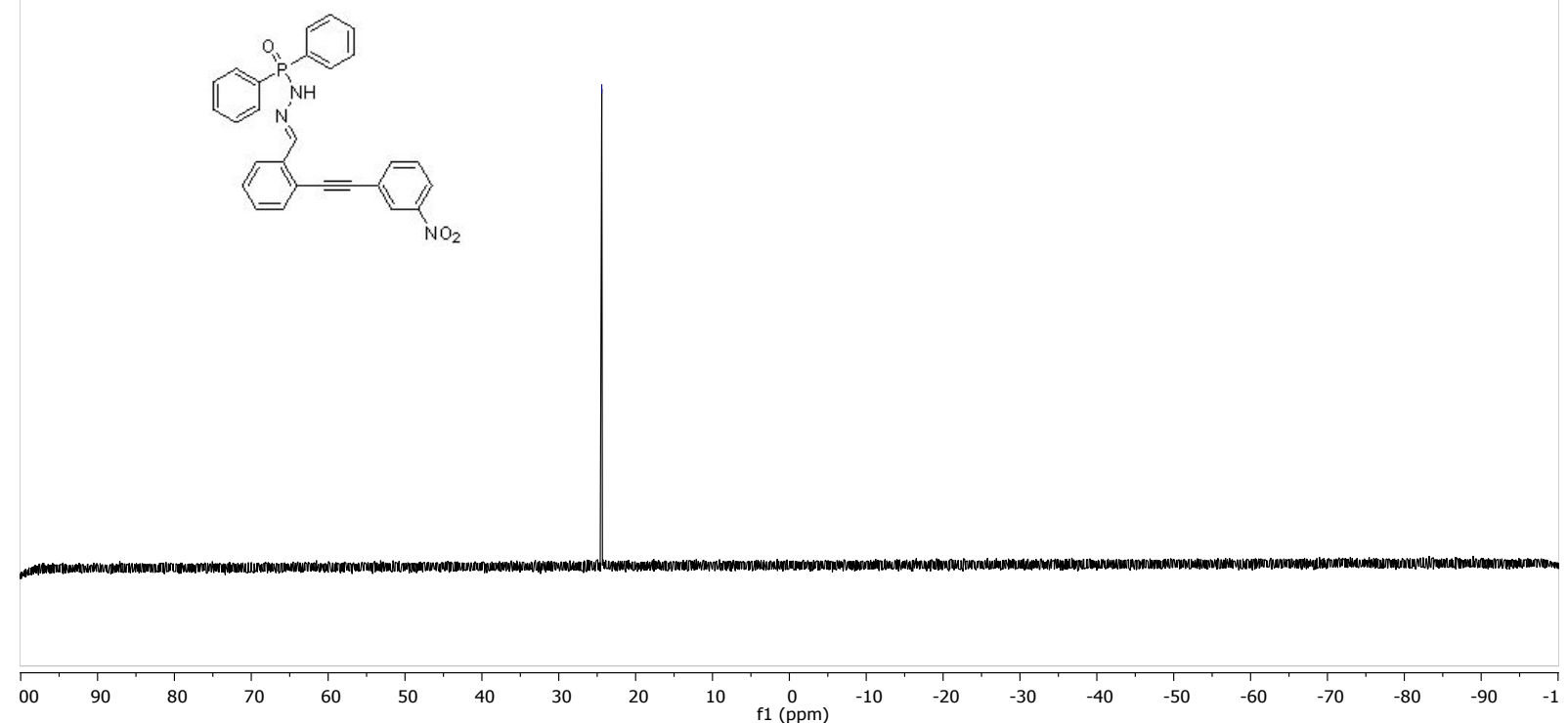


Compound 1y: N'-(5-fluoro-2-(3-methoxyprop-1-yn-1-yl)benzylidene)-P,P-diphenylphosphinic hydrazide

${ }^{1} \mathrm{H}$ NMR (400 MHz, Chloroform-d)

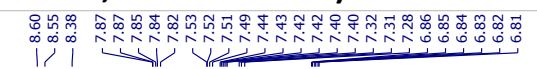
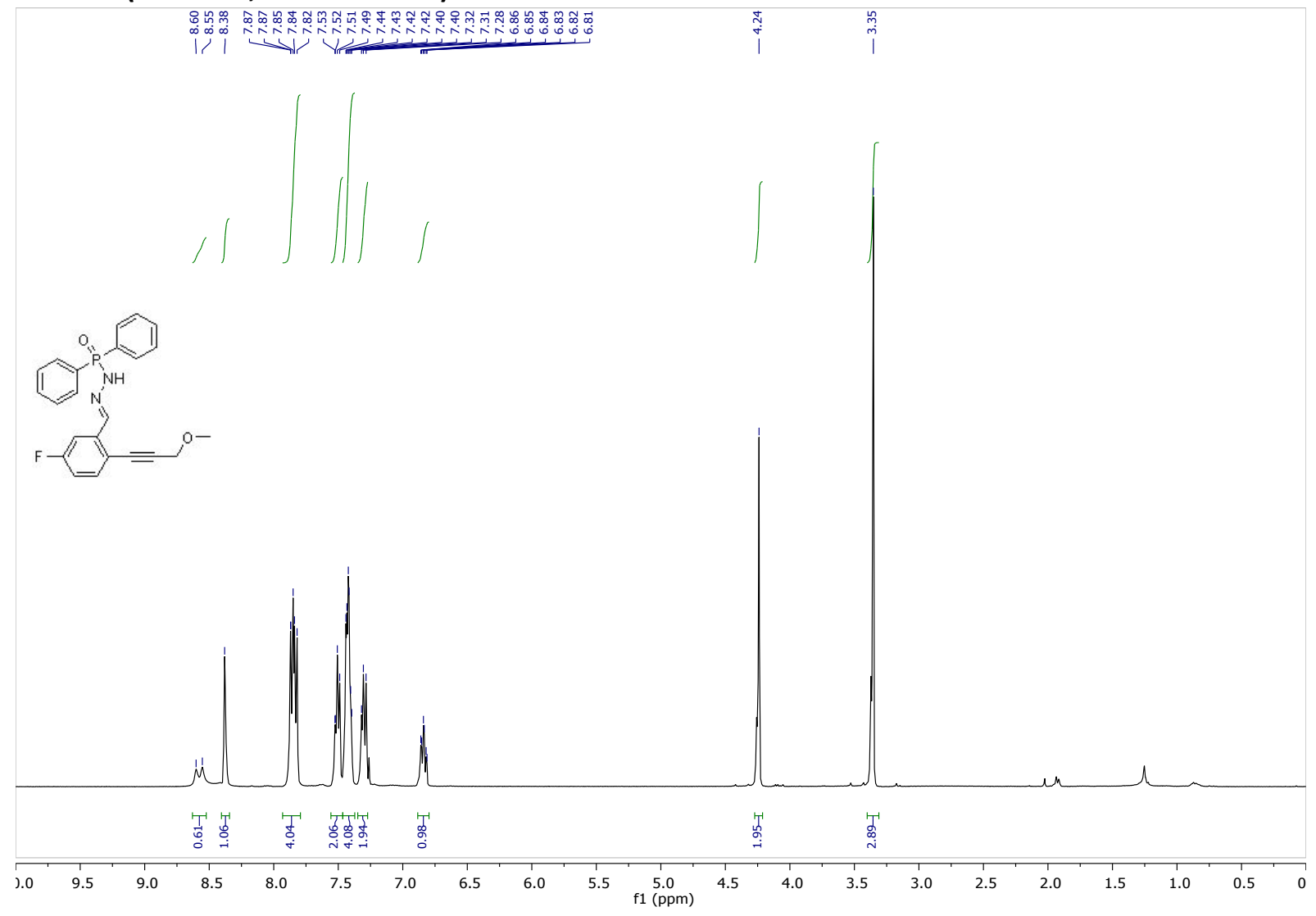

${ }^{13} \mathrm{C}\left\{{ }^{1} \mathrm{H}\right\}$ NMR (101 MHz, Chloroform-d)

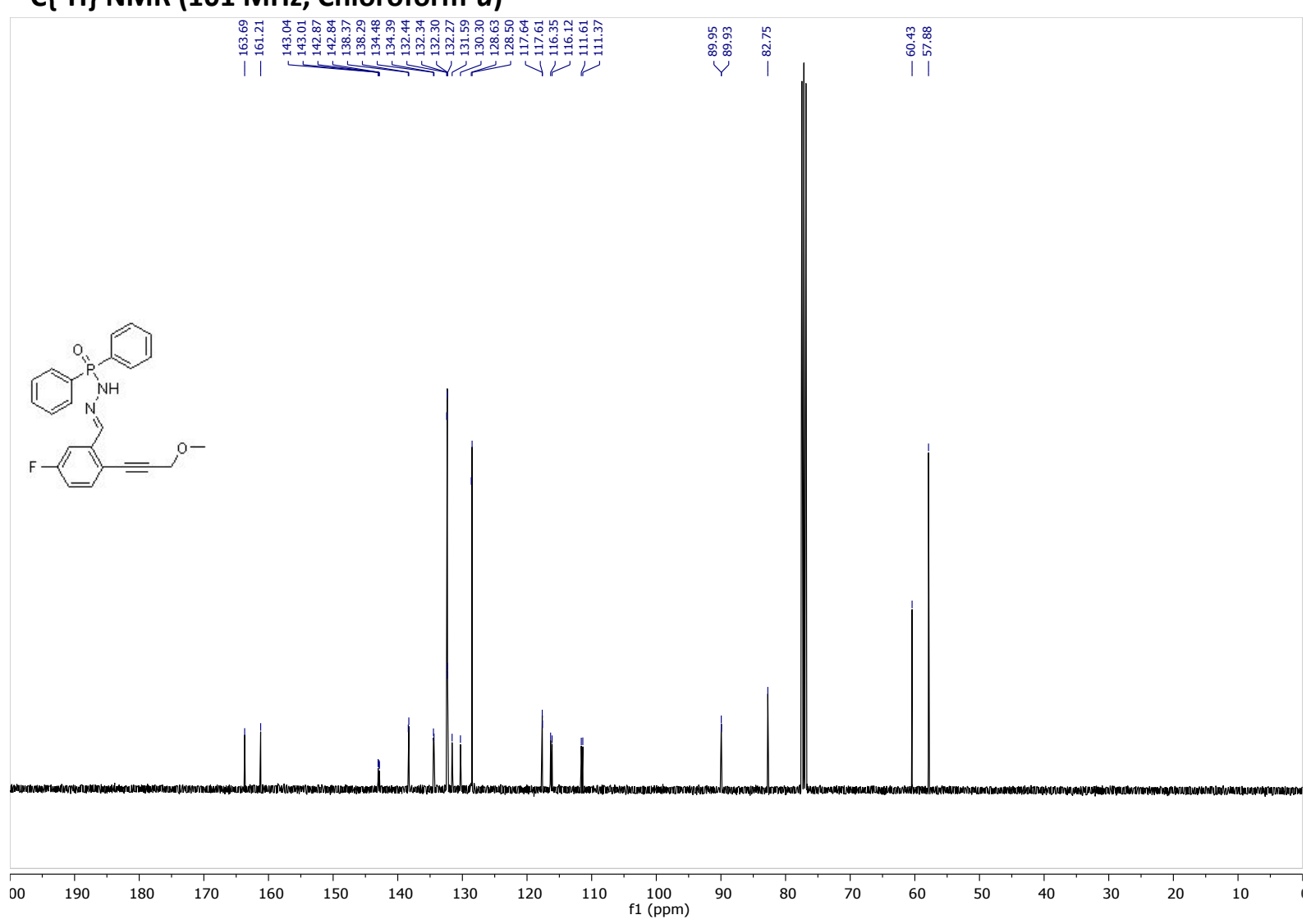

S58 
31P NMR (162 MHz, Chloroform-d)

$\stackrel{\circ}{\circ}$

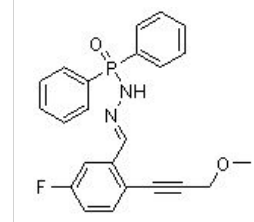

|

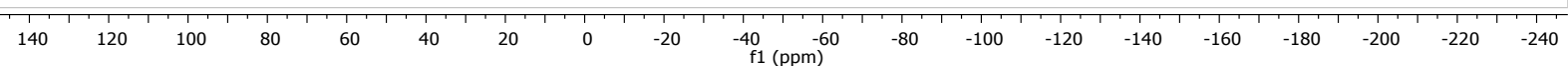

${ }^{19} \mathrm{~F}$ NMR (376 MHz, Chloroform-d)

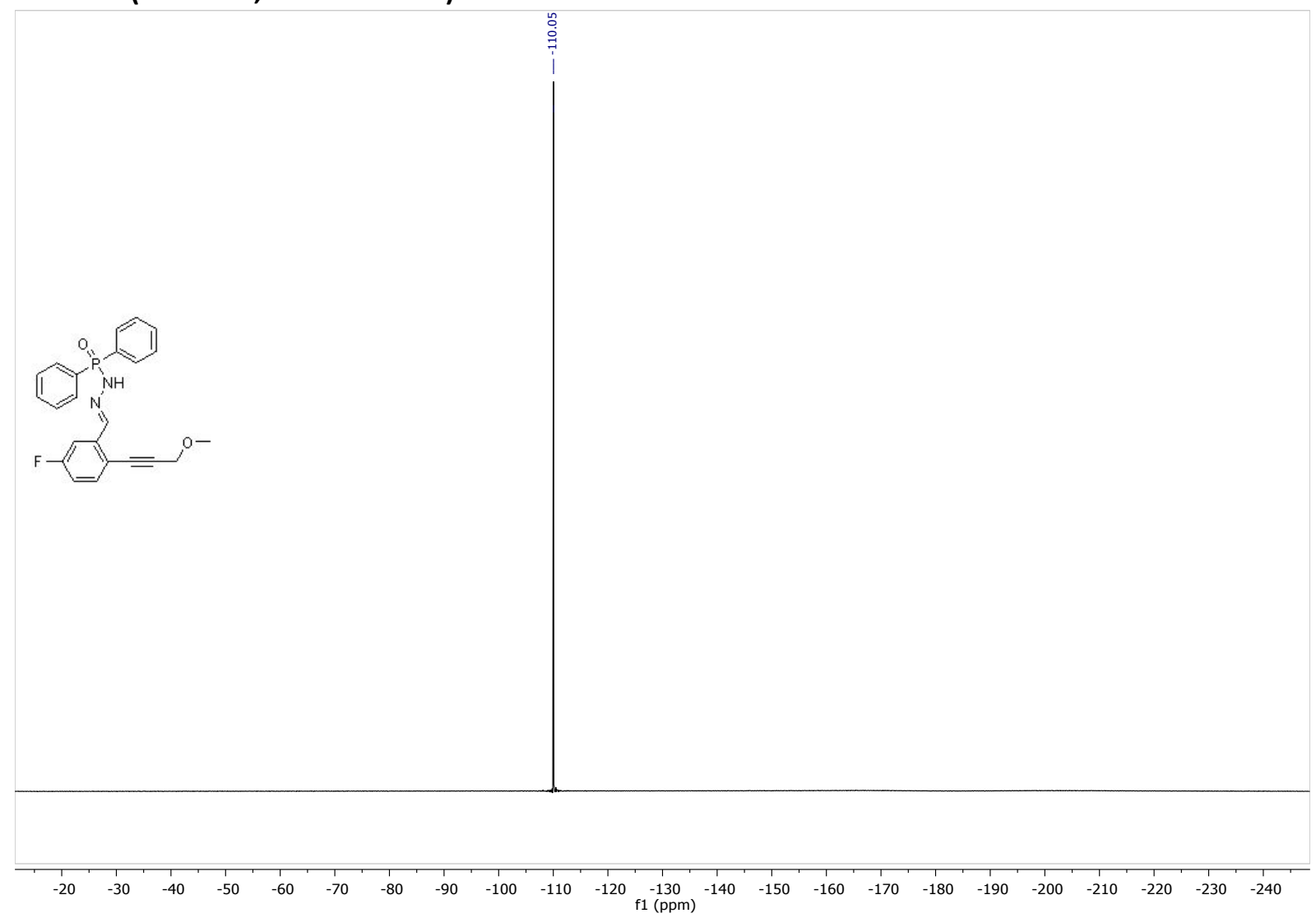


Compound 1z: P,P-diphenyl-N'-(2-((trimethylsilyl)ethynyl)benzylidene)phosphinic hydrazide ${ }^{1} \mathrm{H}$ NMR (300 MHz, Chloroform-d)

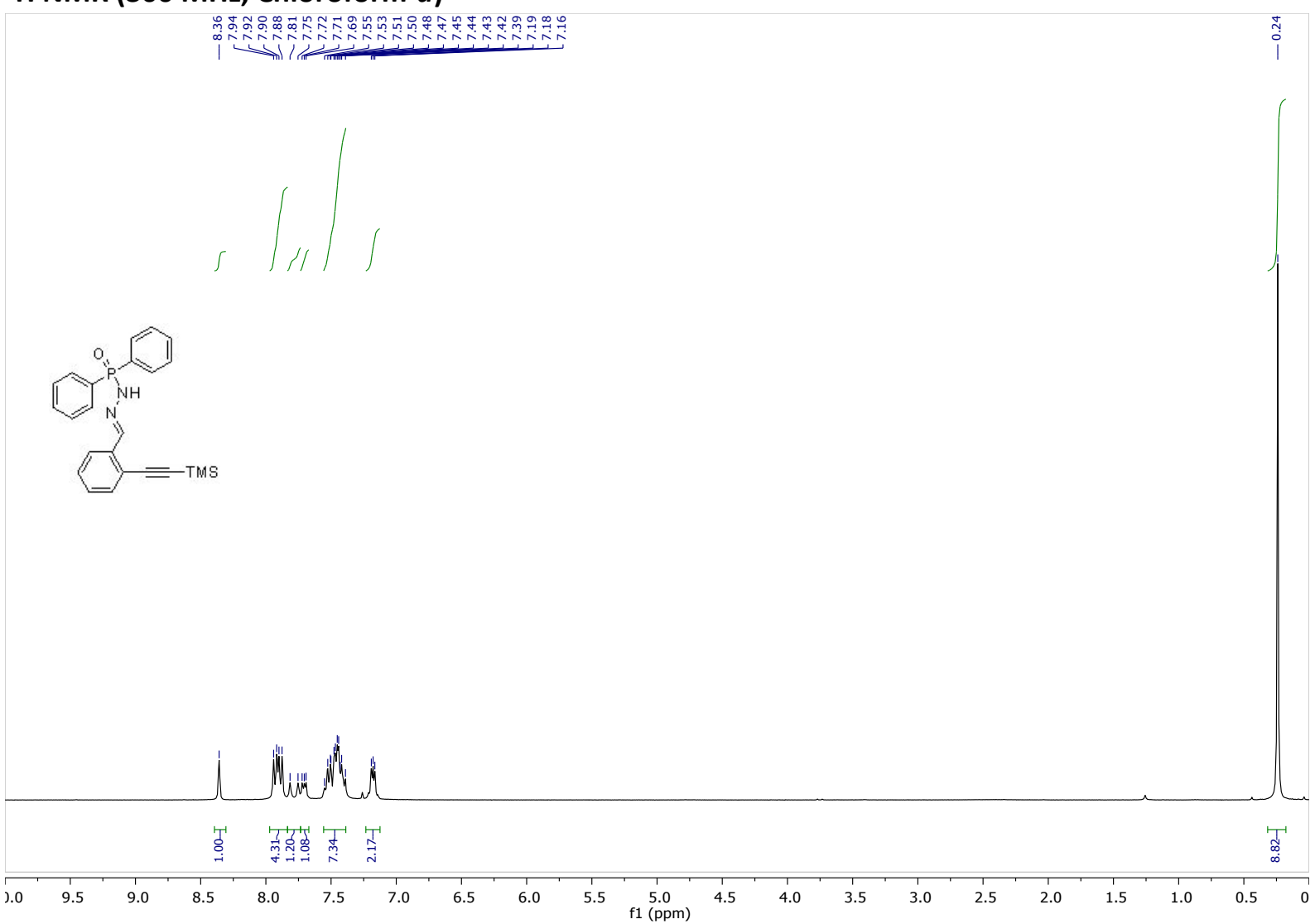

${ }^{13} \mathrm{C}\left\{{ }^{1} \mathrm{H}\right\}$ NMR (75 MHz, Chloroform-d)

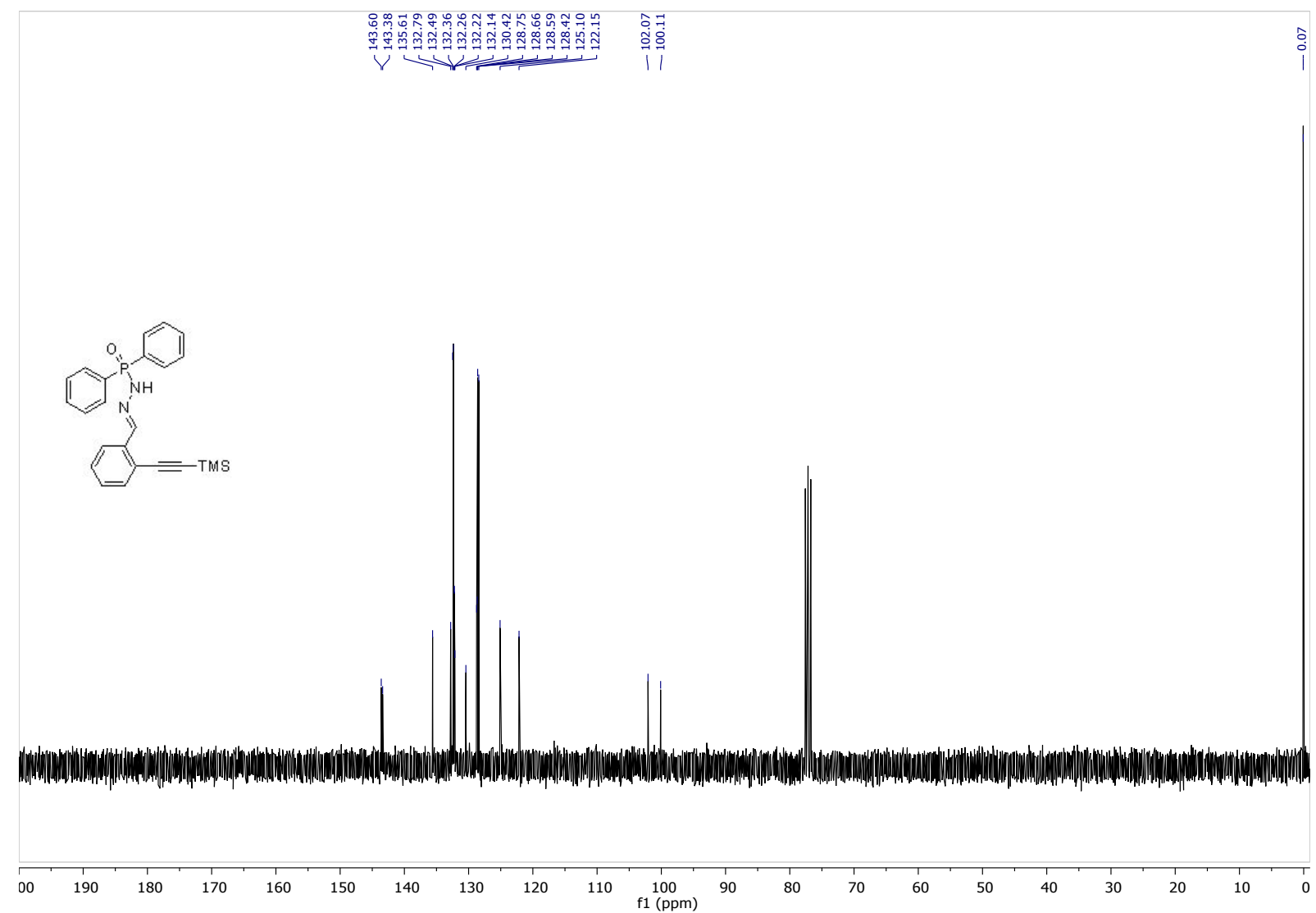


31P NMR (121 MHz, Chloroform-d)

\section{$\stackrel{\substack{7 \\ \sim}}{\sim}$}
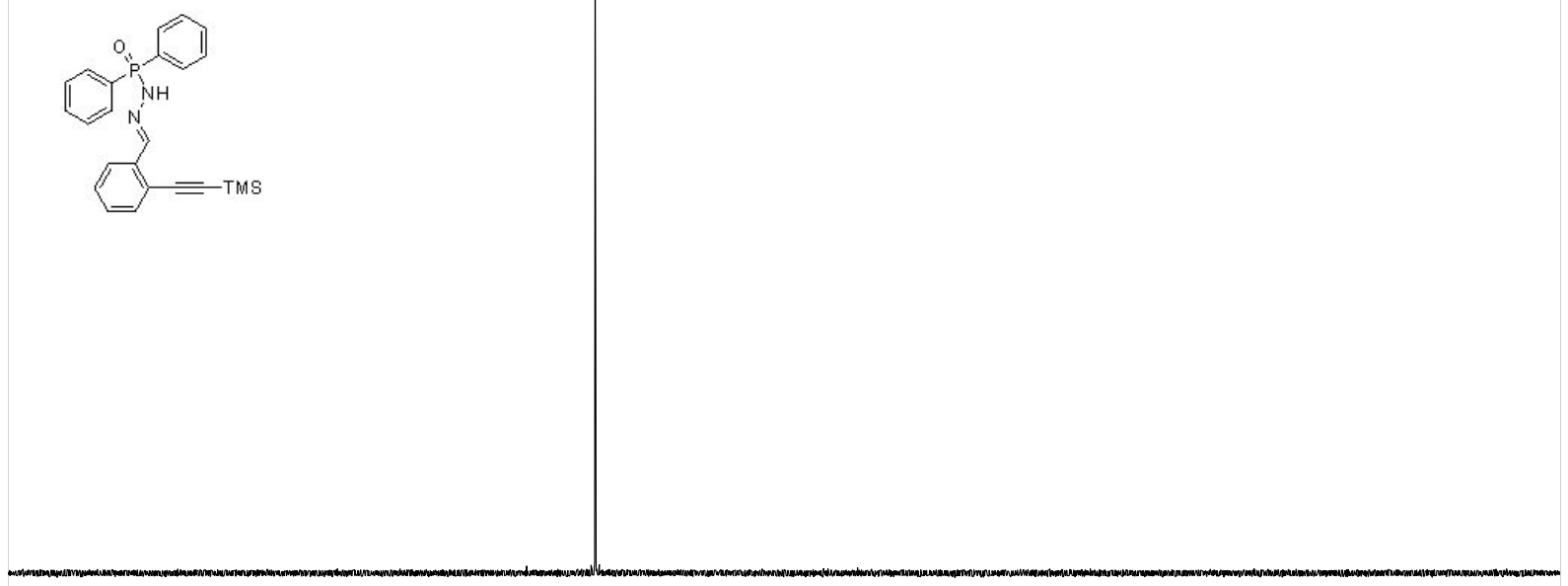

\begin{tabular}{llllllllllllllllllllllllll}
\hline 0 & 90 & 80 & 70 & 60 & 50 & 40 & 30 & 20 & 10 & 0 & -10 & -20 & -30 & -40 & -50 & -60 & -70 & -80 & -90 & -1
\end{tabular} 
VI- Spectra of phthalazines

\section{Compound 2a: 1-benzhydrylphthalazine}

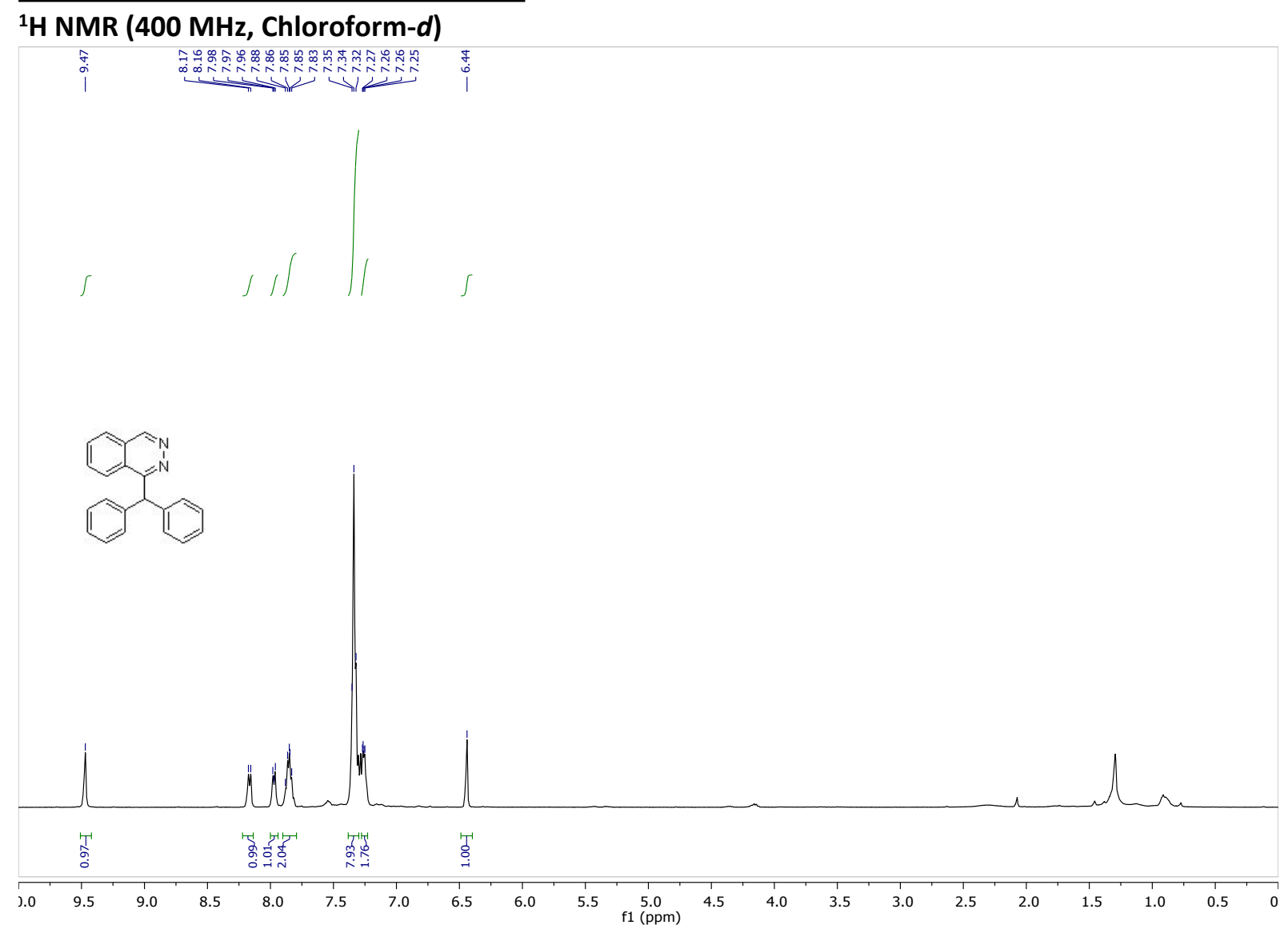


Compound 2b: 1-(phenyl(p-tolyl)methyl)phthalazine

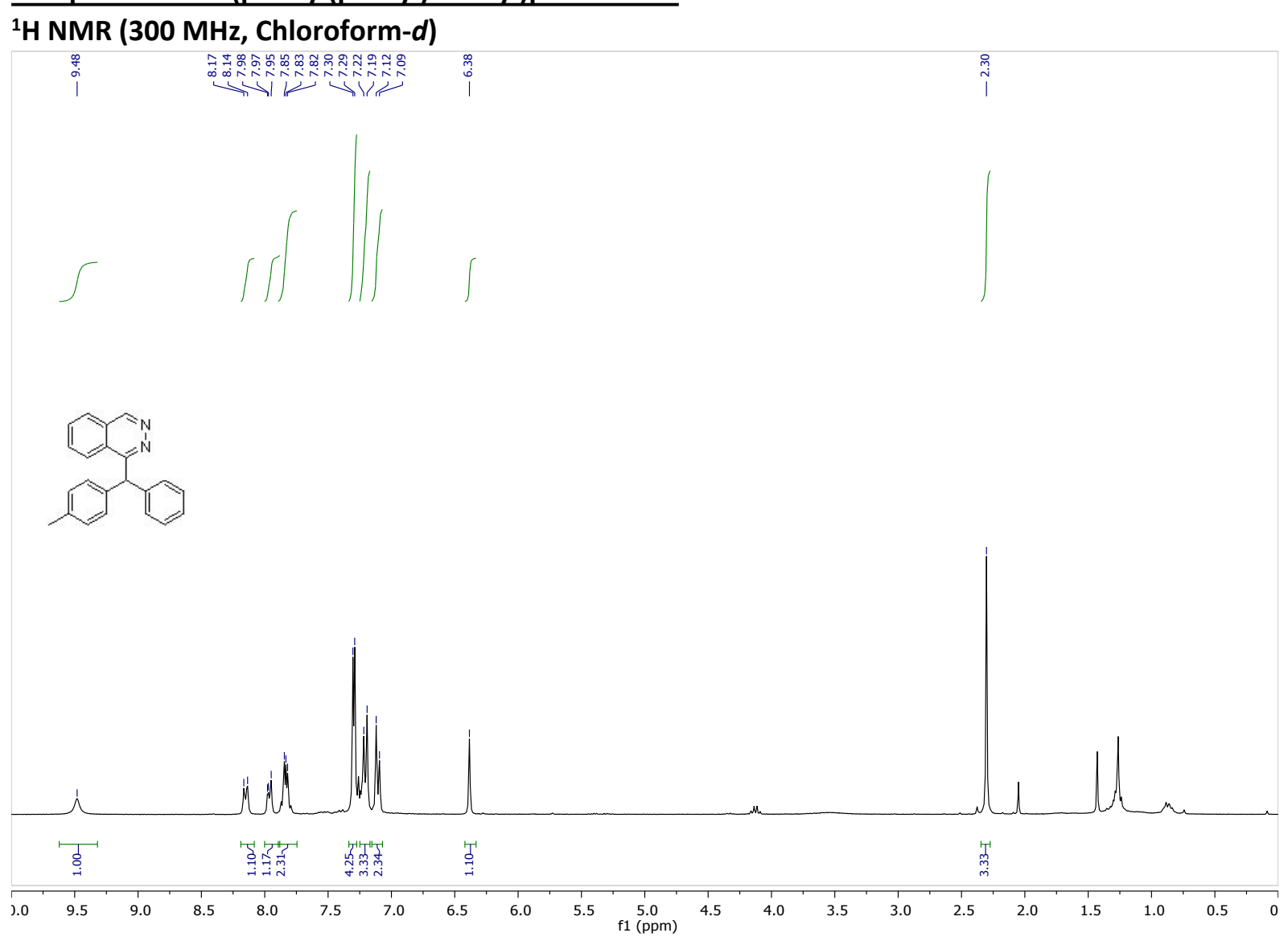

S63 
Compound 2c: 1-((4-fluorophenyl)(phenyl)methyl)phthalazine

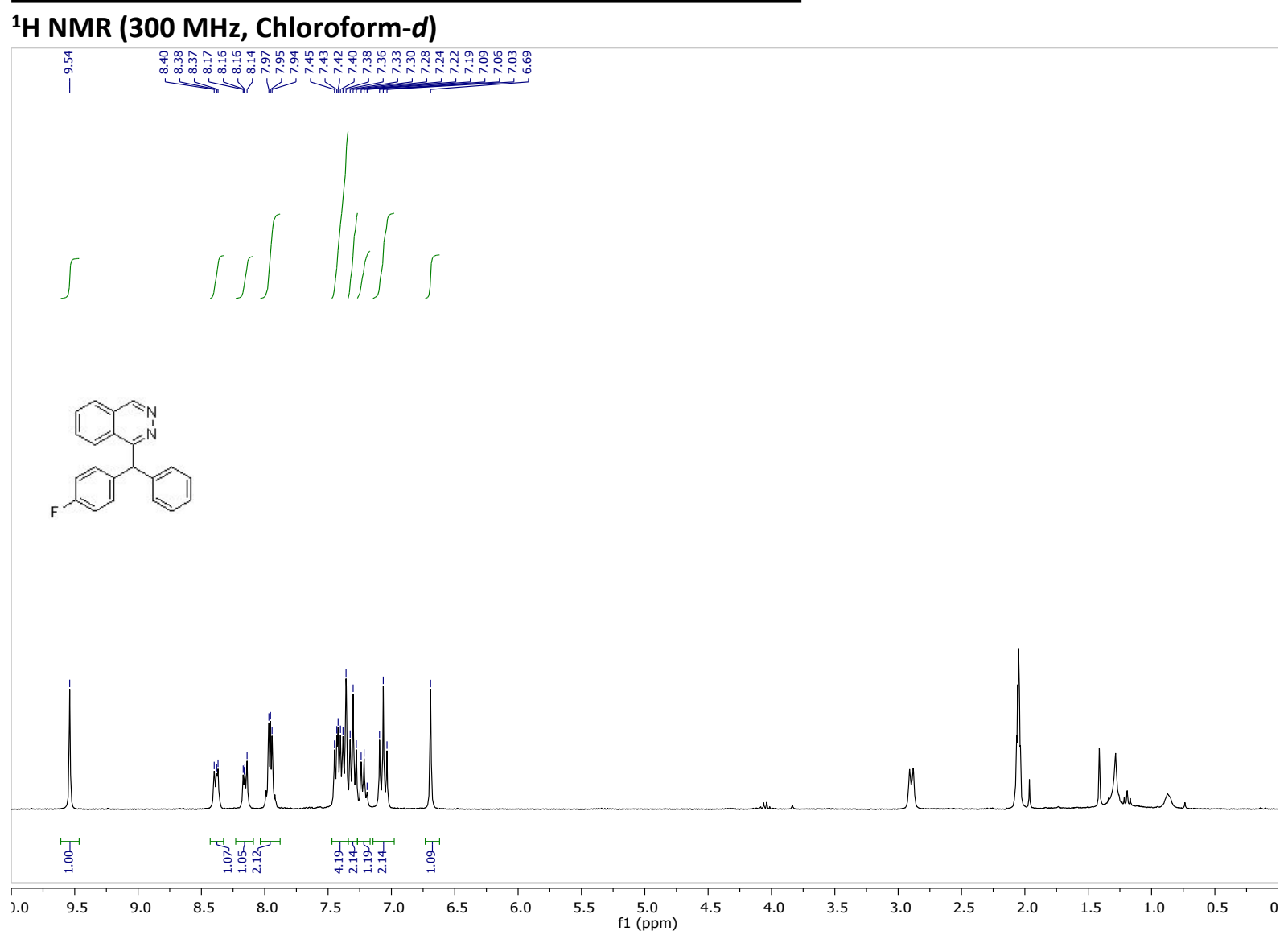

S64 
Compound 2d: 1-((6-methoxynaphthalen-2-yl)(phenyl)methyl)phthalazine

${ }^{1} \mathrm{H}$ NMR (400 MHz, Chloroform-d)

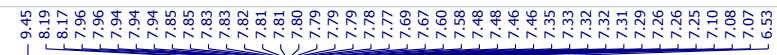

$\stackrel{\infty}{i}$

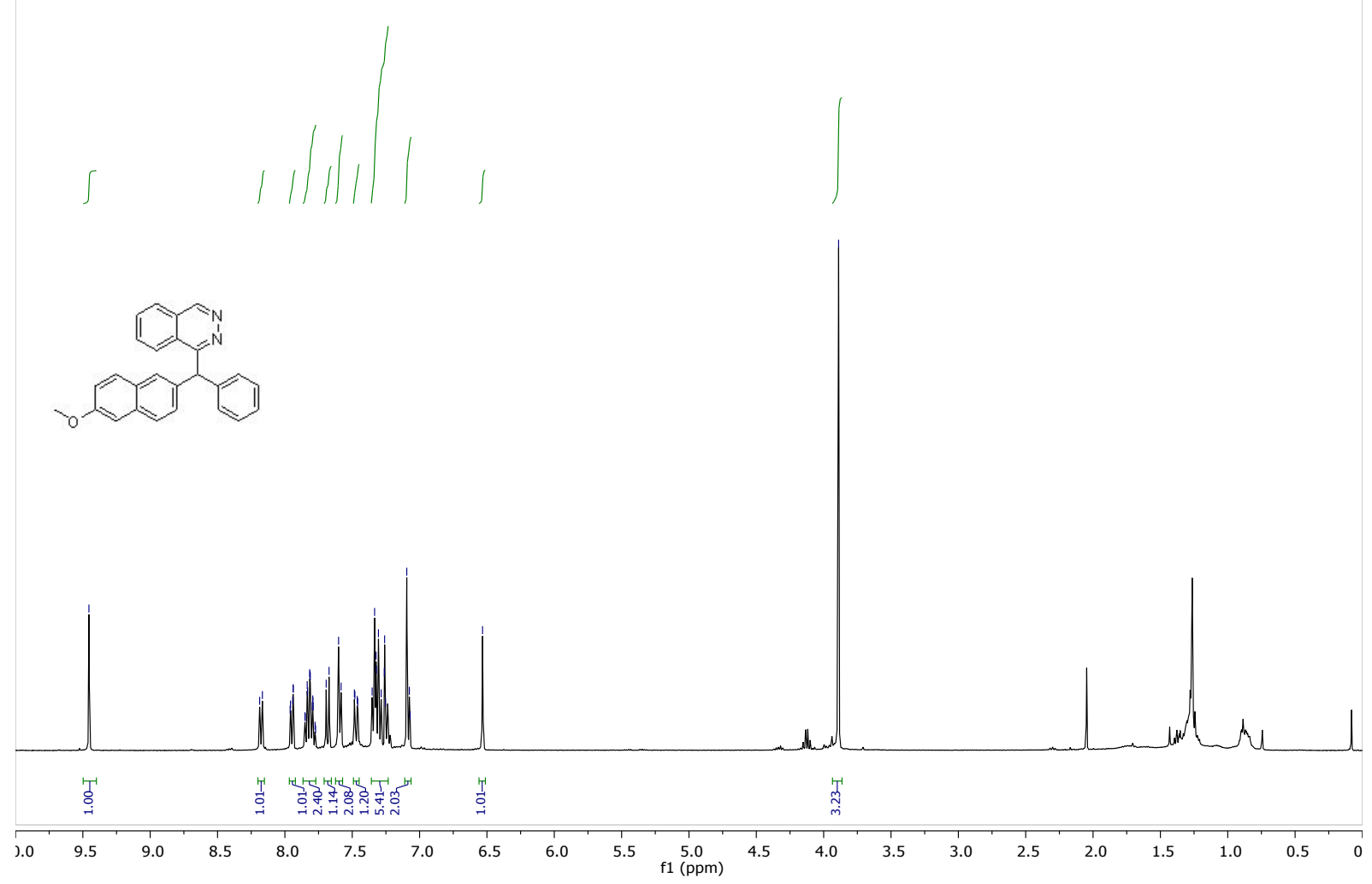

${ }^{13} \mathrm{C}\left\{{ }^{1} \mathrm{H}\right\}$ NMR (101 MHz, Chloroform-d)

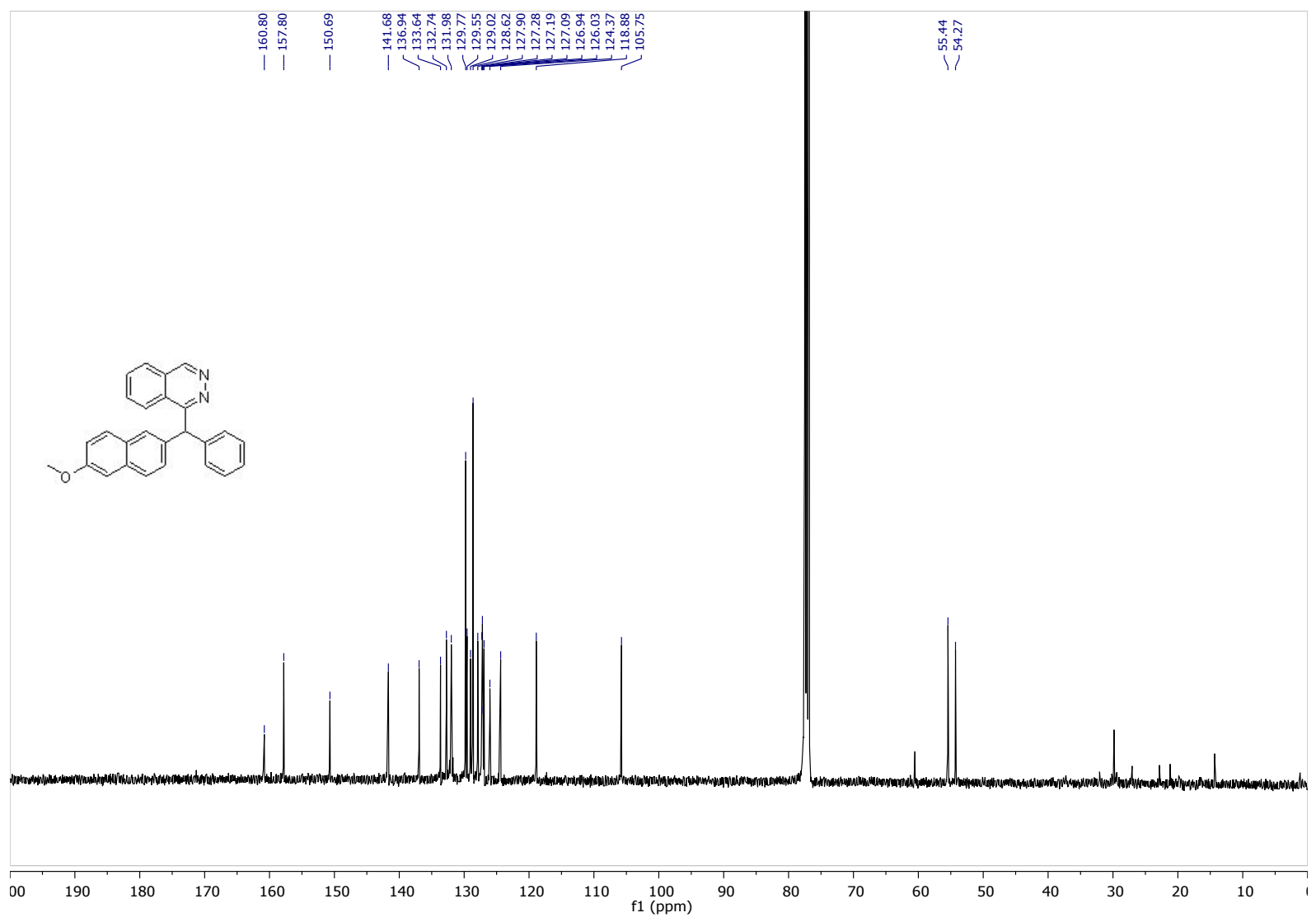

S65 
Compound 2e: 1-(dibenzo[b,d]thiophen-2-yl(phenyl)methyl)phthalazine

${ }^{1} \mathrm{H}$ NMR (300 MHz, Chloroform-d)

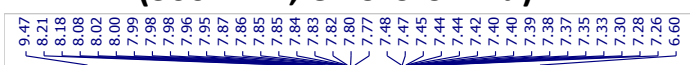

s
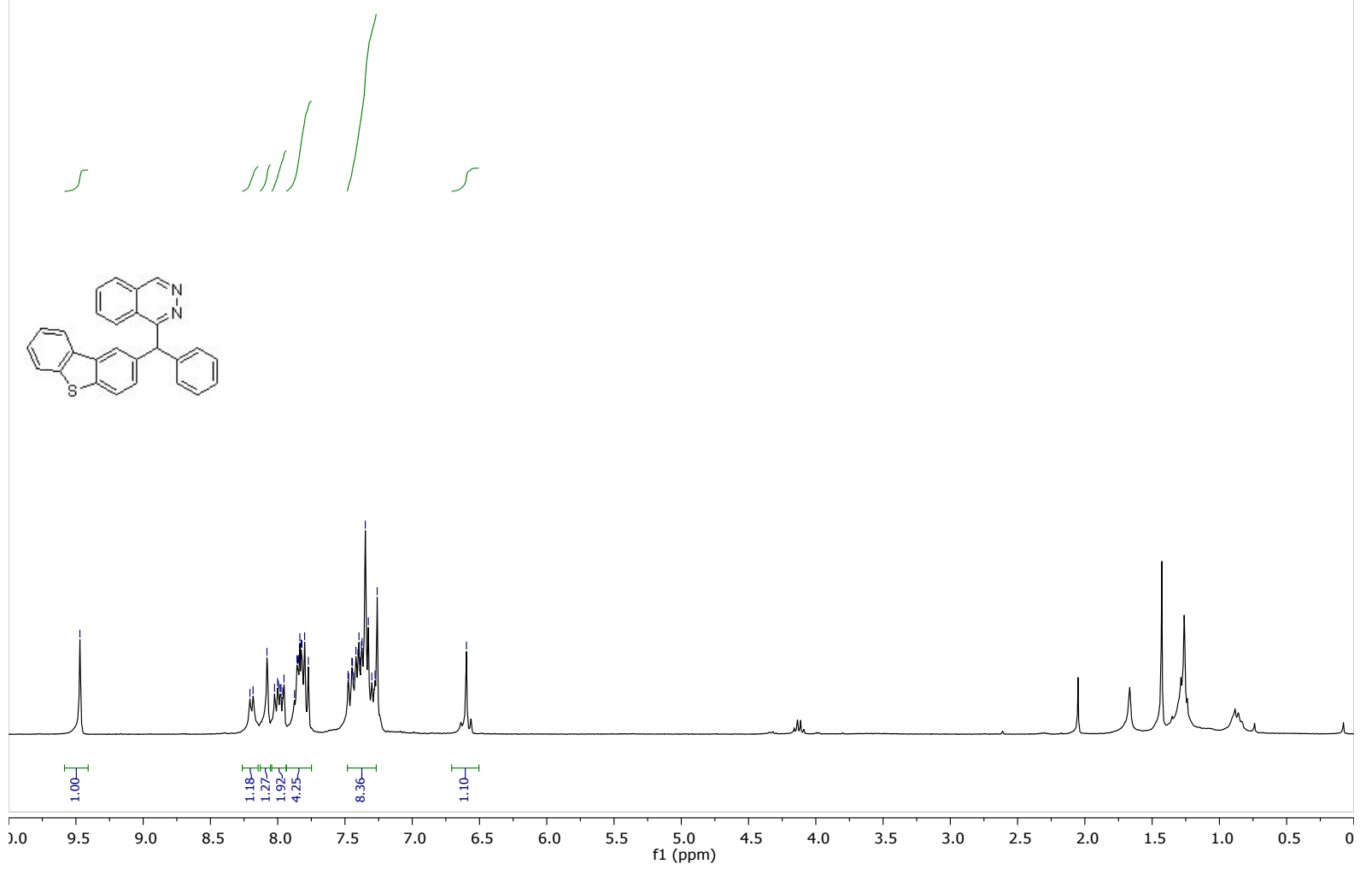

${ }^{13} \mathrm{C}\left\{{ }^{1} \mathrm{H}\right\}$ NMR (75 MHz, Chloroform-d)

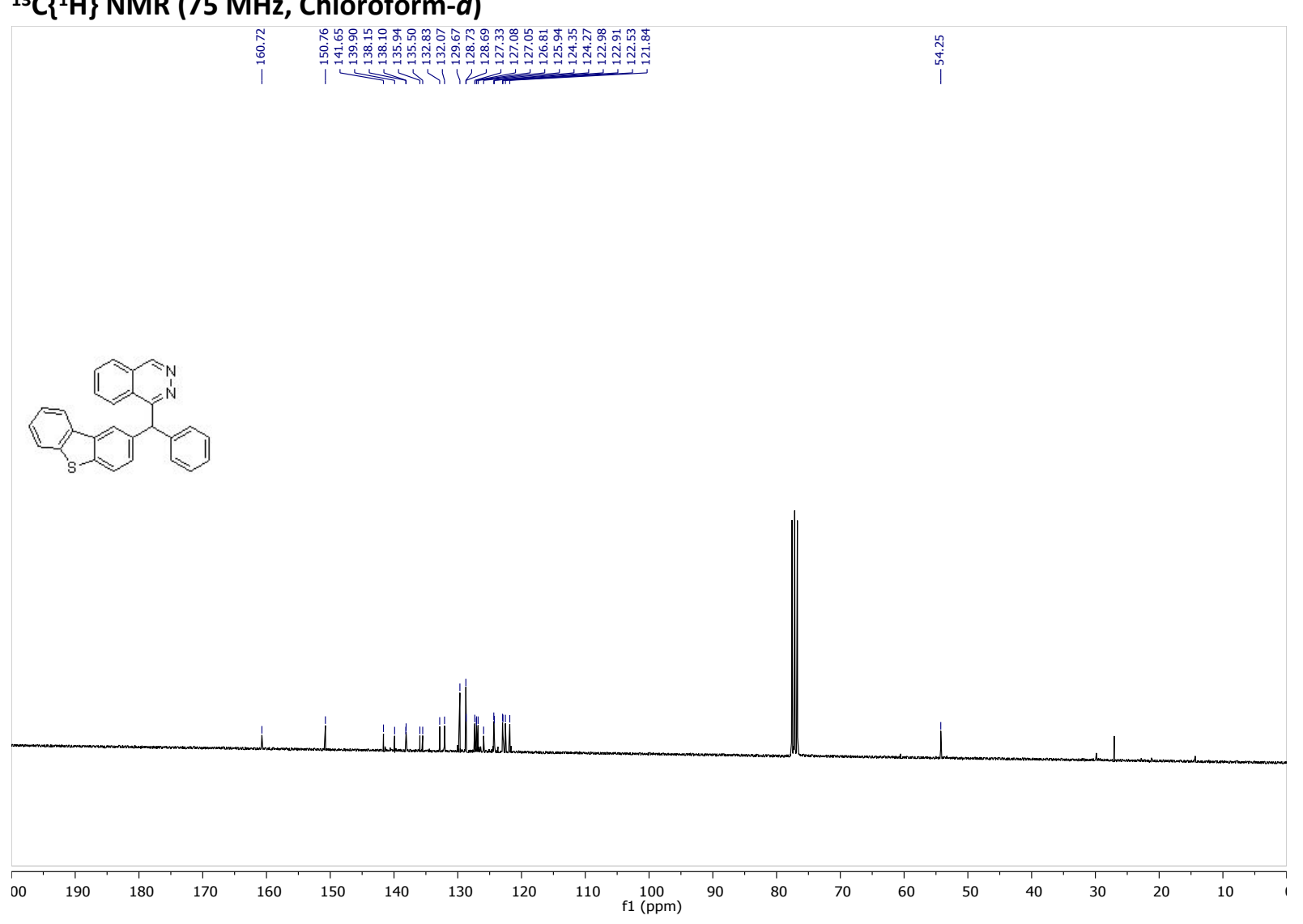

S67 


\section{Compound 2f: 1-(1-phenylbutyl)phthalazine}

${ }^{1} \mathrm{H}$ NMR (400 MHz, Chloroform-d)

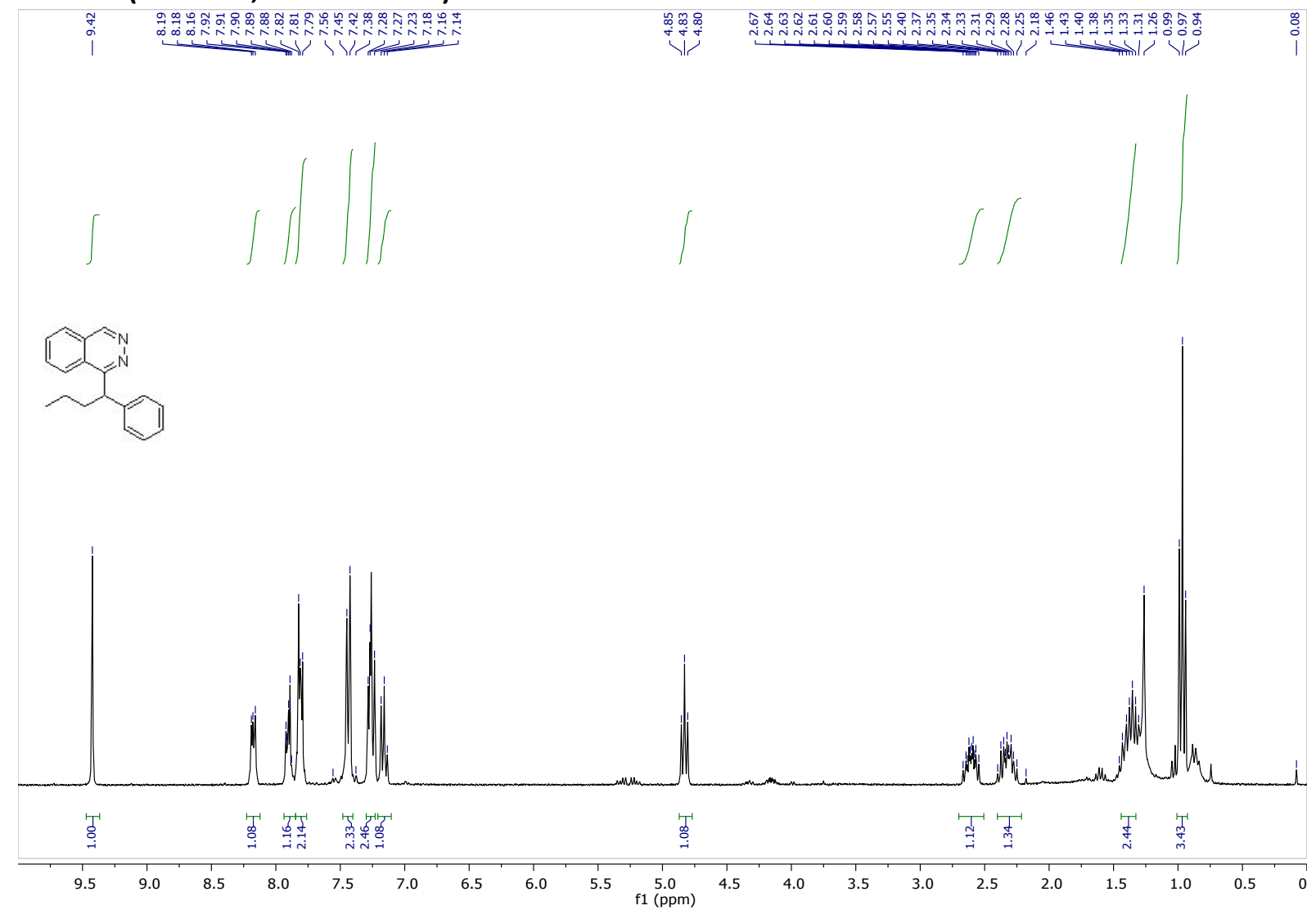




\section{Compound 2h: 1-benzhydryl-6,7,8-trimethoxyphthalazine}

${ }^{1} \mathrm{H}$ NMR (300 MHz, Chloroform-d)

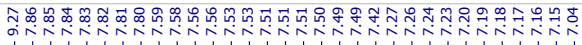

1ึi

$\int$

$\iint \|_{1}$
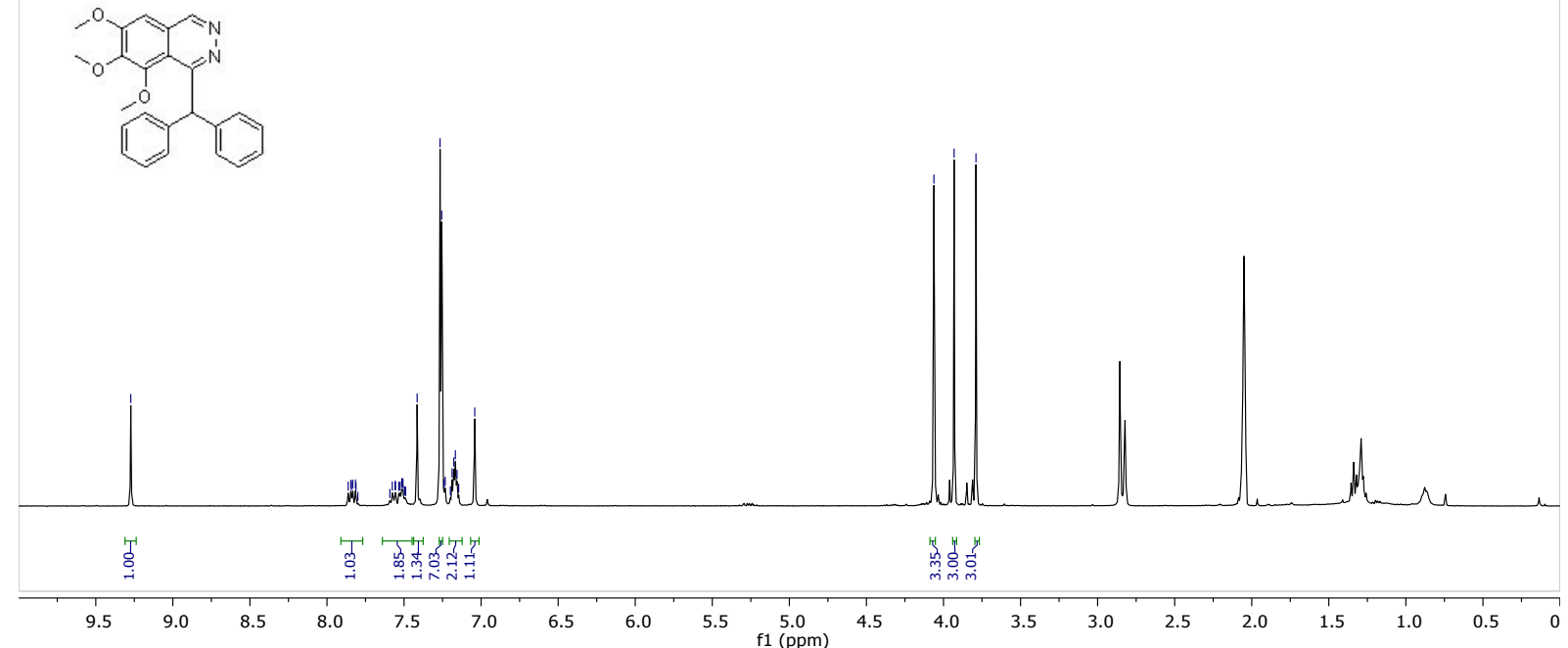
Compound 2i: 1-benzhydryl-6-fluorophthalazine

${ }^{1}$ H NMR (400 MHz, Chloroform-d)

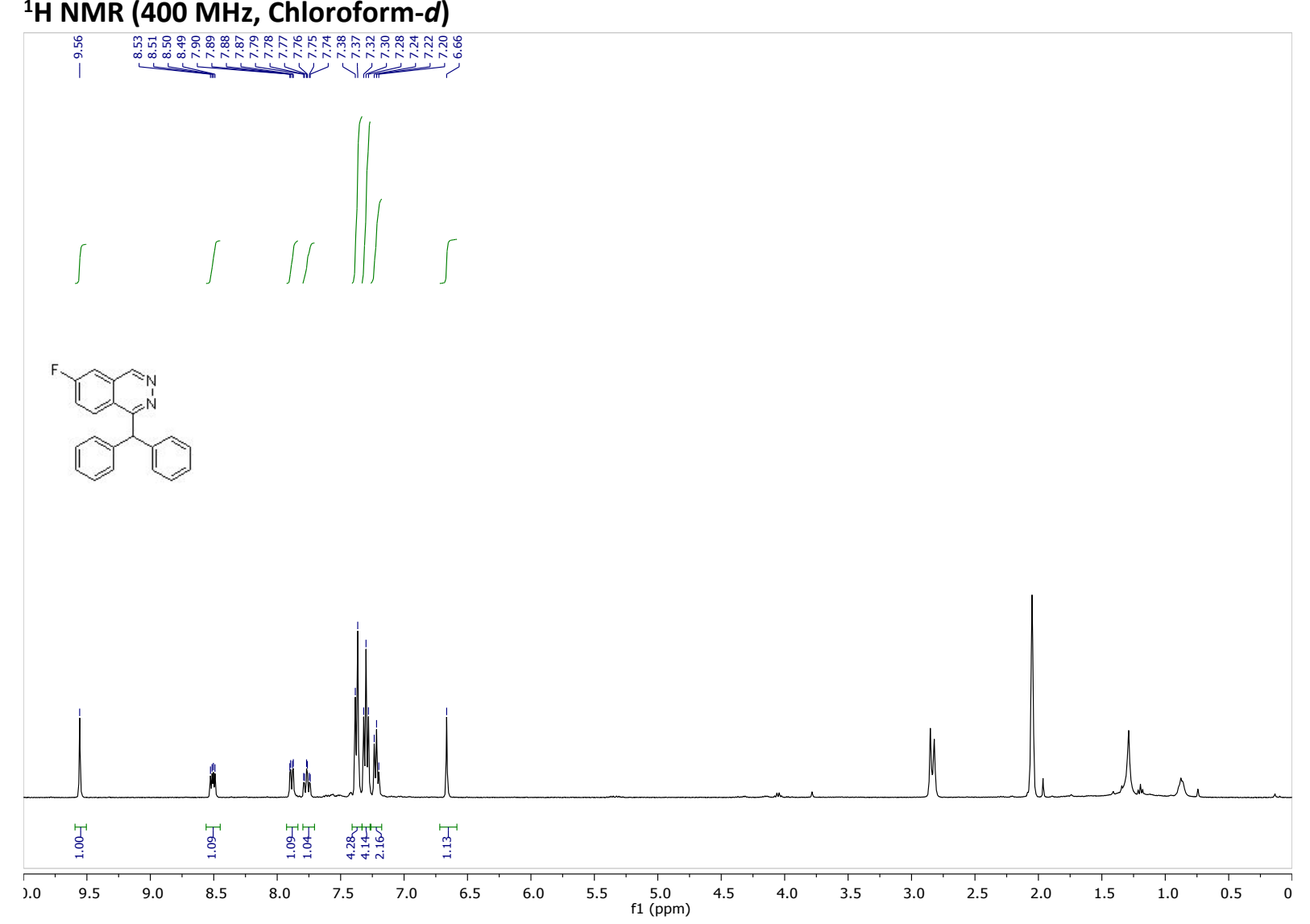

S70 
Compound 2j: 1-benzhydryl-6-chlorophthalazine

${ }^{1}$ H NMR (400 MHz, Chloroform-d)

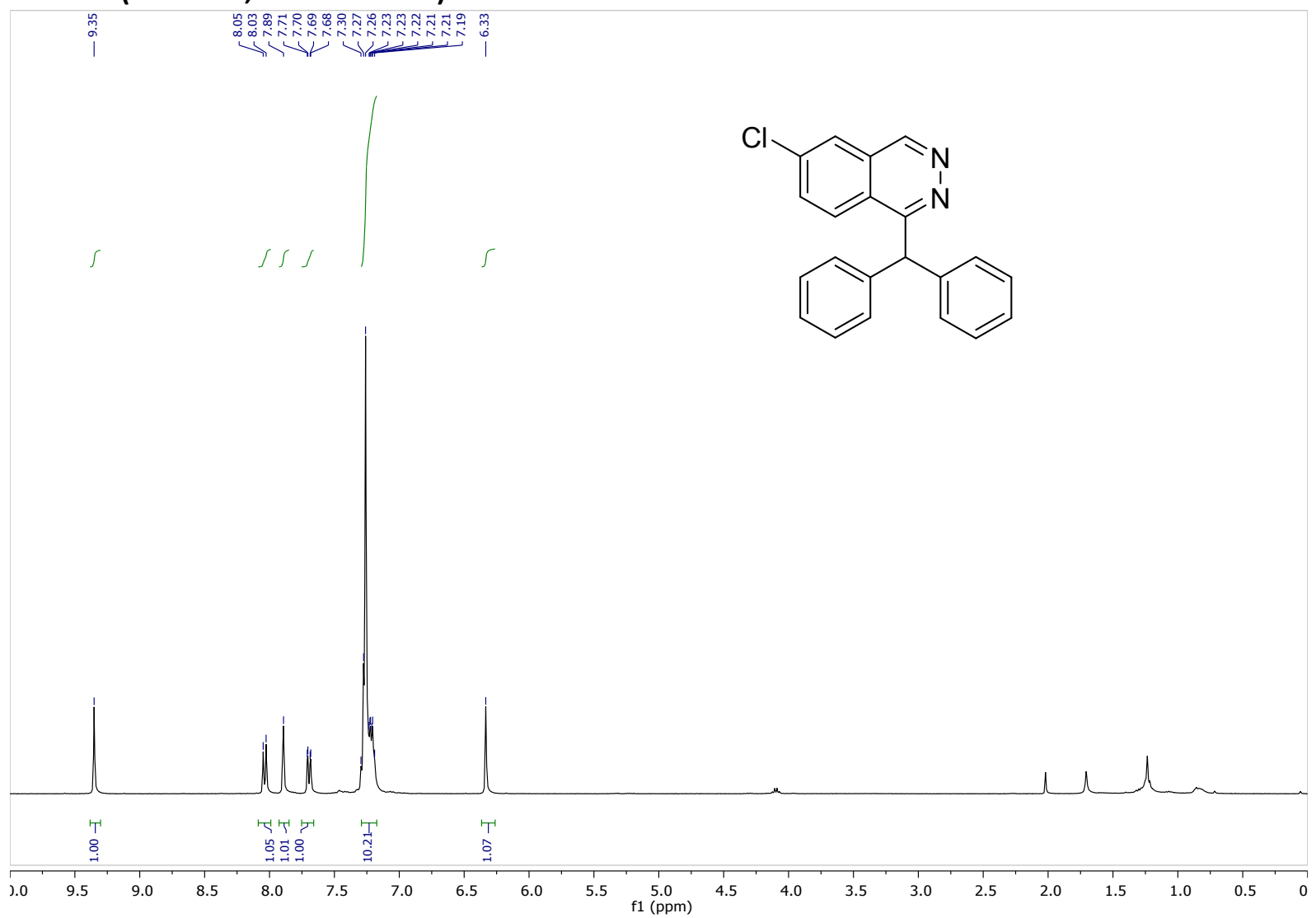

${ }^{13} \mathrm{C}\left\{{ }^{1} \mathrm{H}\right\}$ NMR (101 MHz, Chloroform-d)

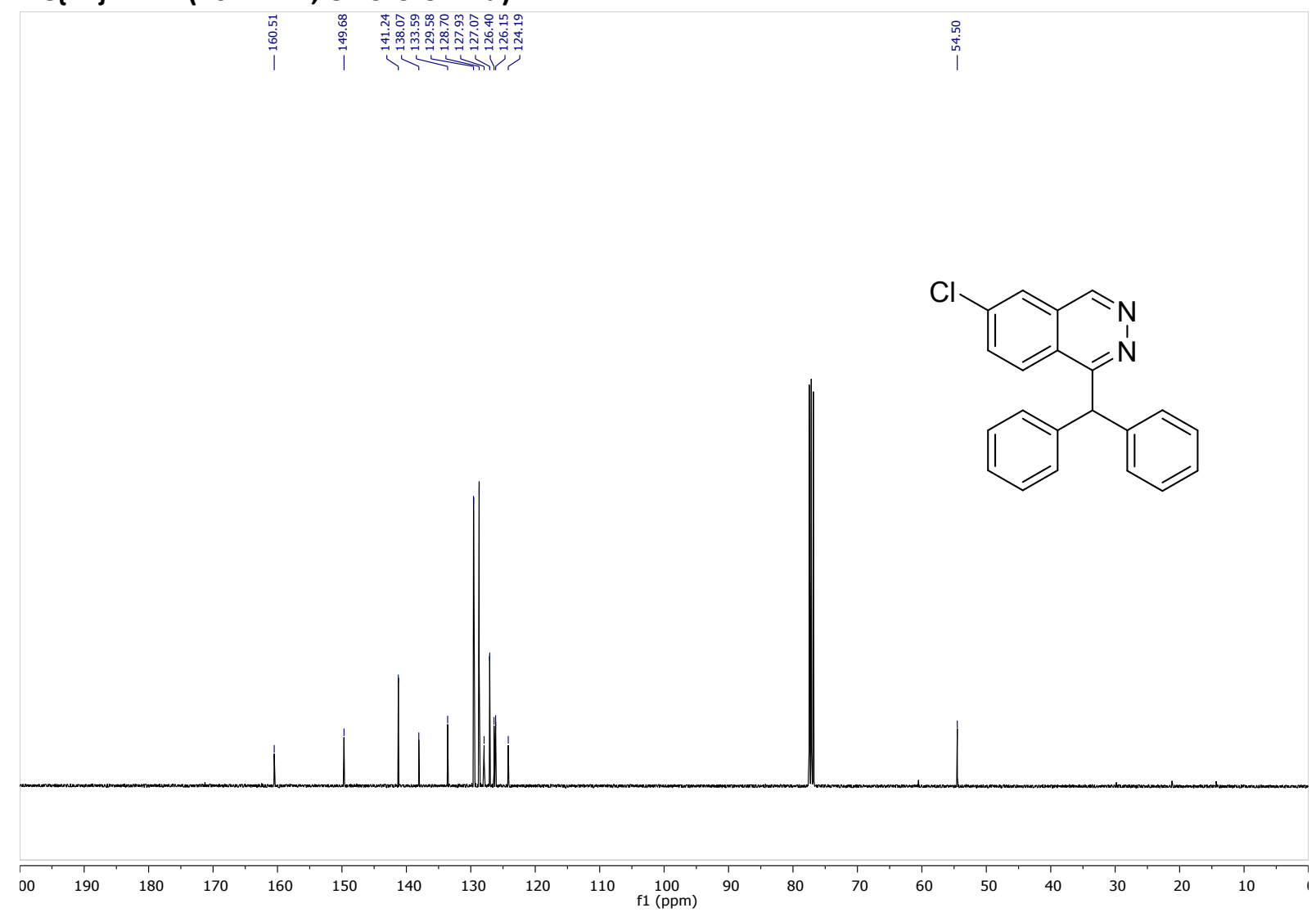

S71 
Compound 2k: 1-benzhydryl-6-bromophthalazine

${ }^{1}$ H NMR (400 MHz, Chloroform-d)

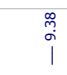

$\underbrace{7}$
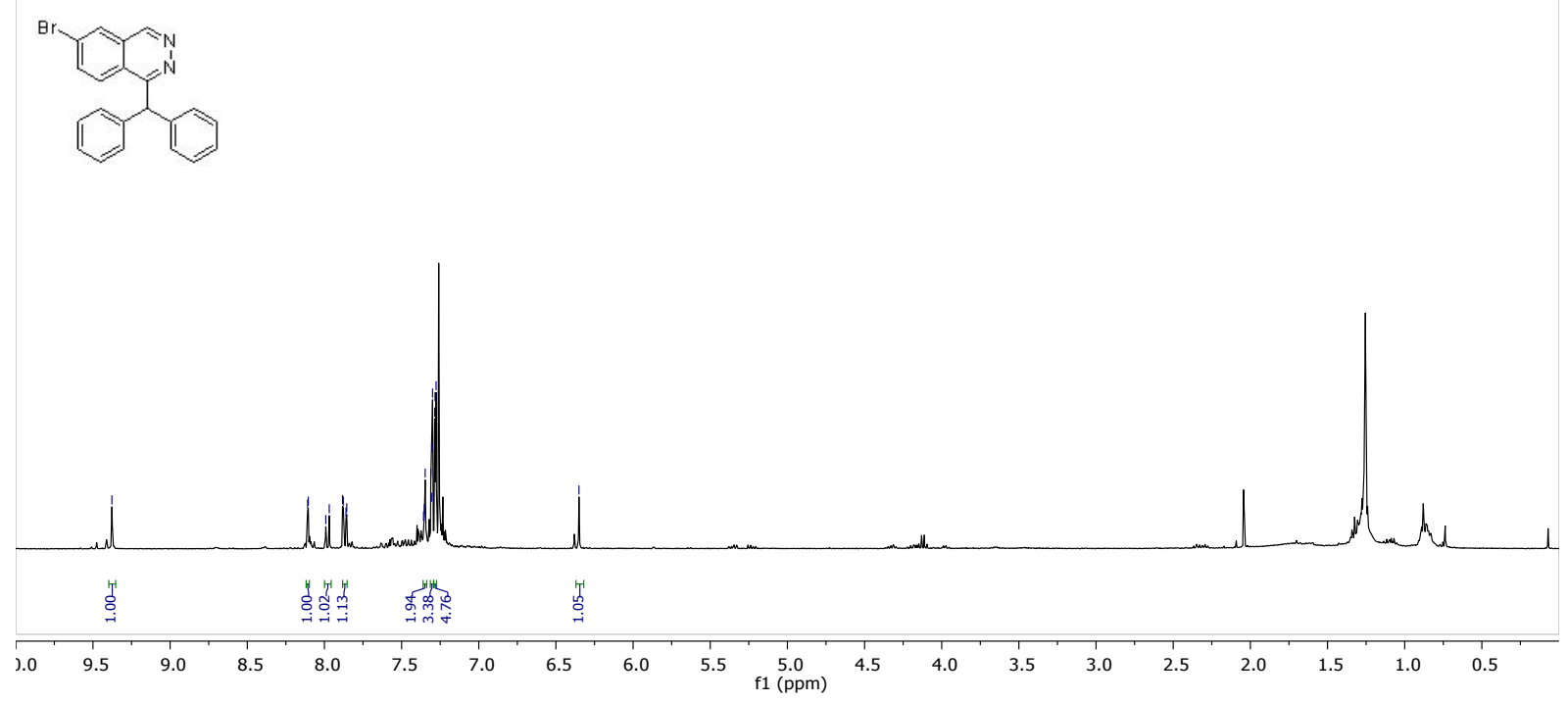

${ }^{13} \mathrm{C}\left\{{ }^{1} \mathrm{H}\right\}$ NMR (101 MHz, Chloroform-d)

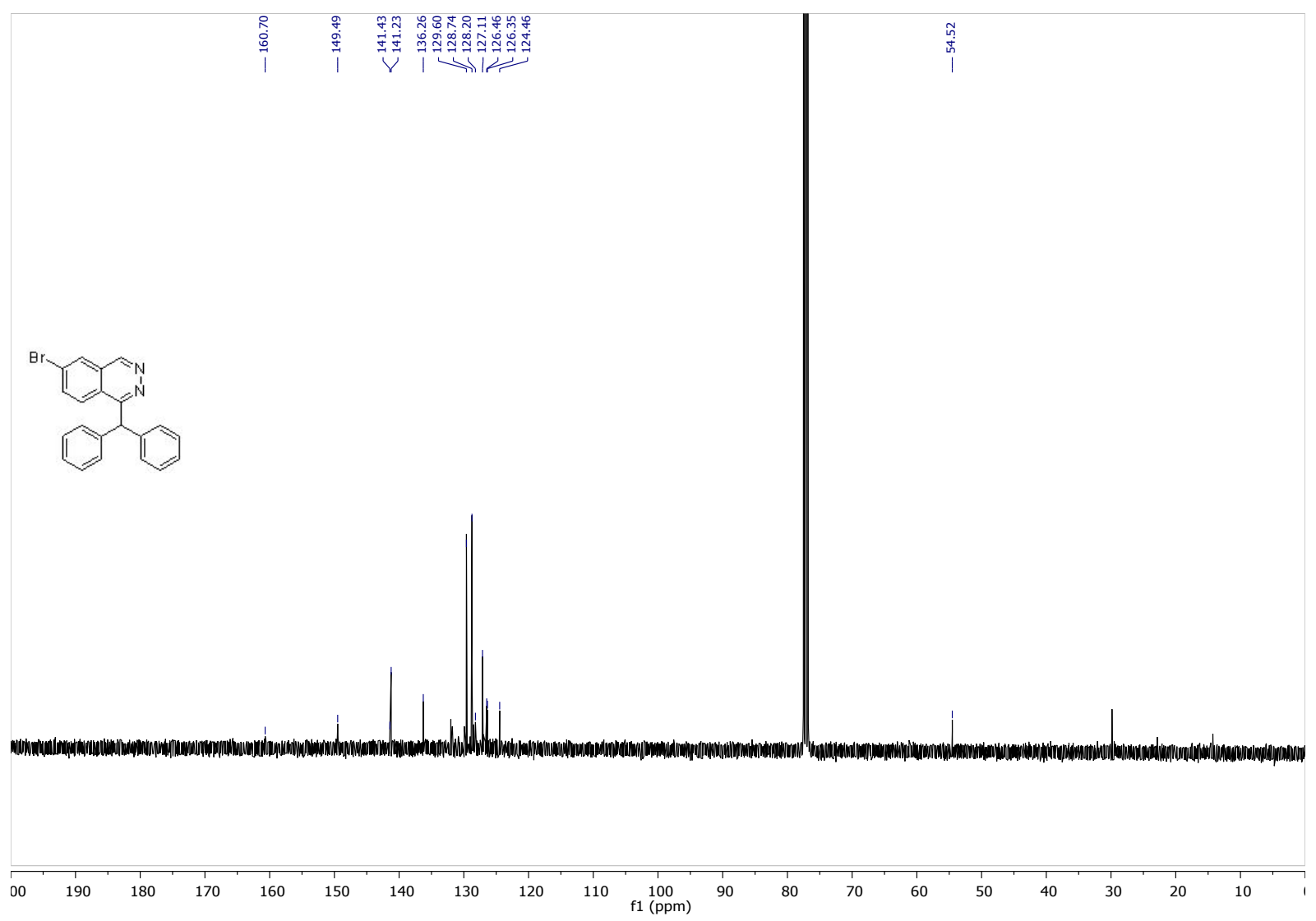

S72 
Compound 2m: 4-benzhydryl-5-methyl-5H-pyridazino[4,5-b]indole

${ }^{1} \mathrm{H}$ NMR (400 MHz, Chloroform-d)
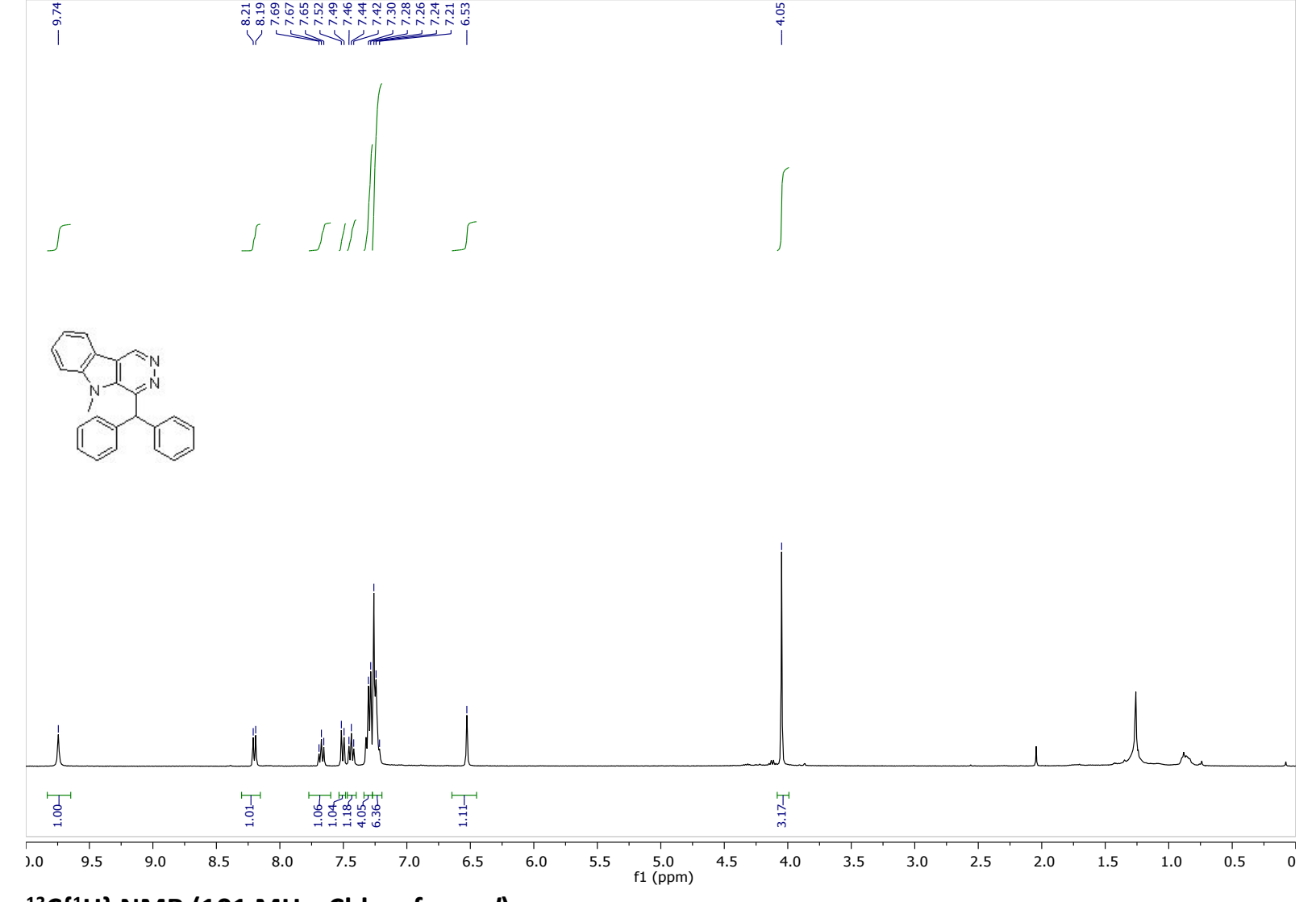

${ }^{13} \mathrm{C}\left\{{ }^{1} \mathrm{H}\right\}$ NMR (101 MHz, Chloroform-d)

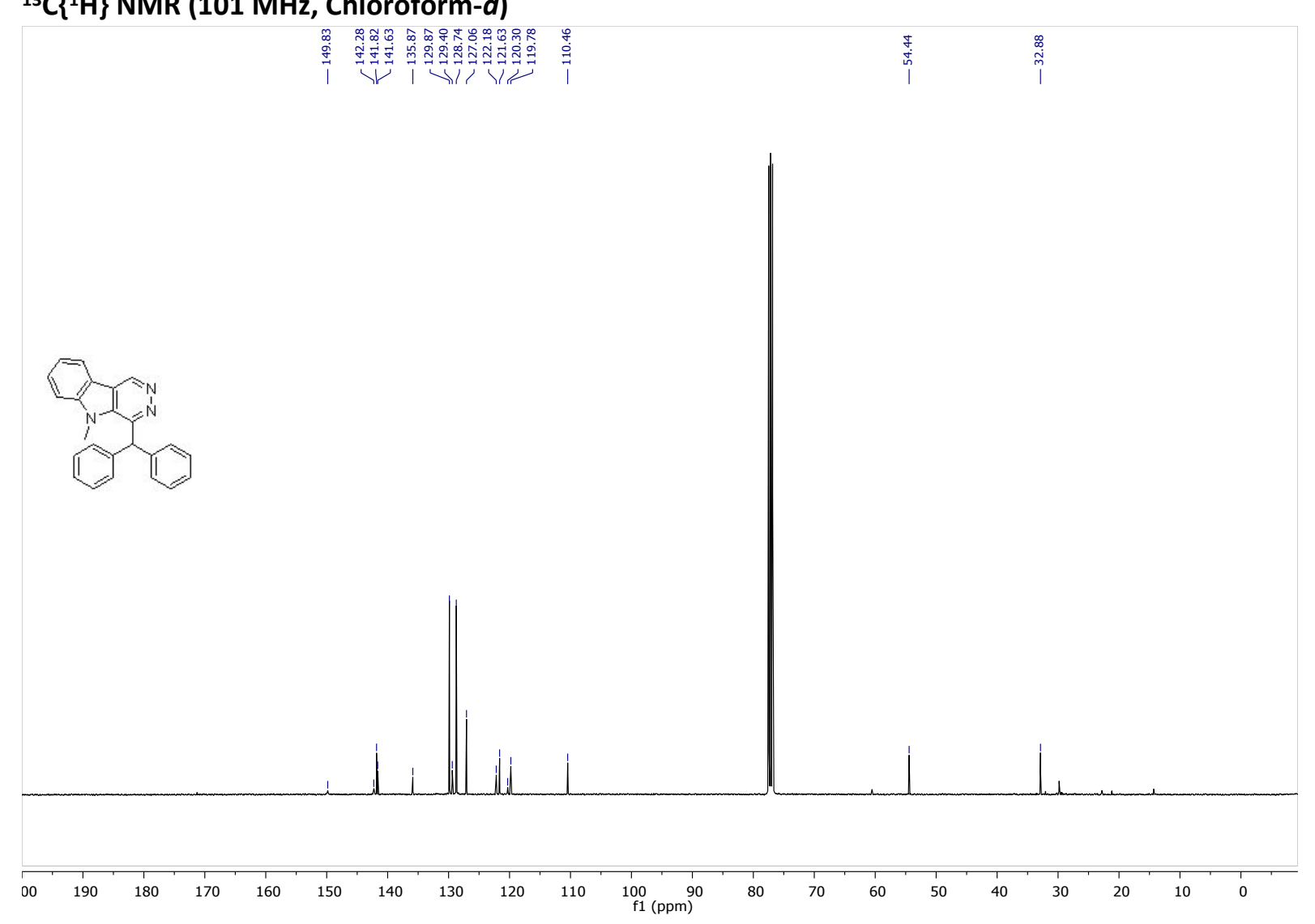

S74 
Compound 2n: 4-benzhydrylbenzofuro[2,3-d]pyridazine

${ }^{1}$ H NMR (400 MHz, Chloroform-d)

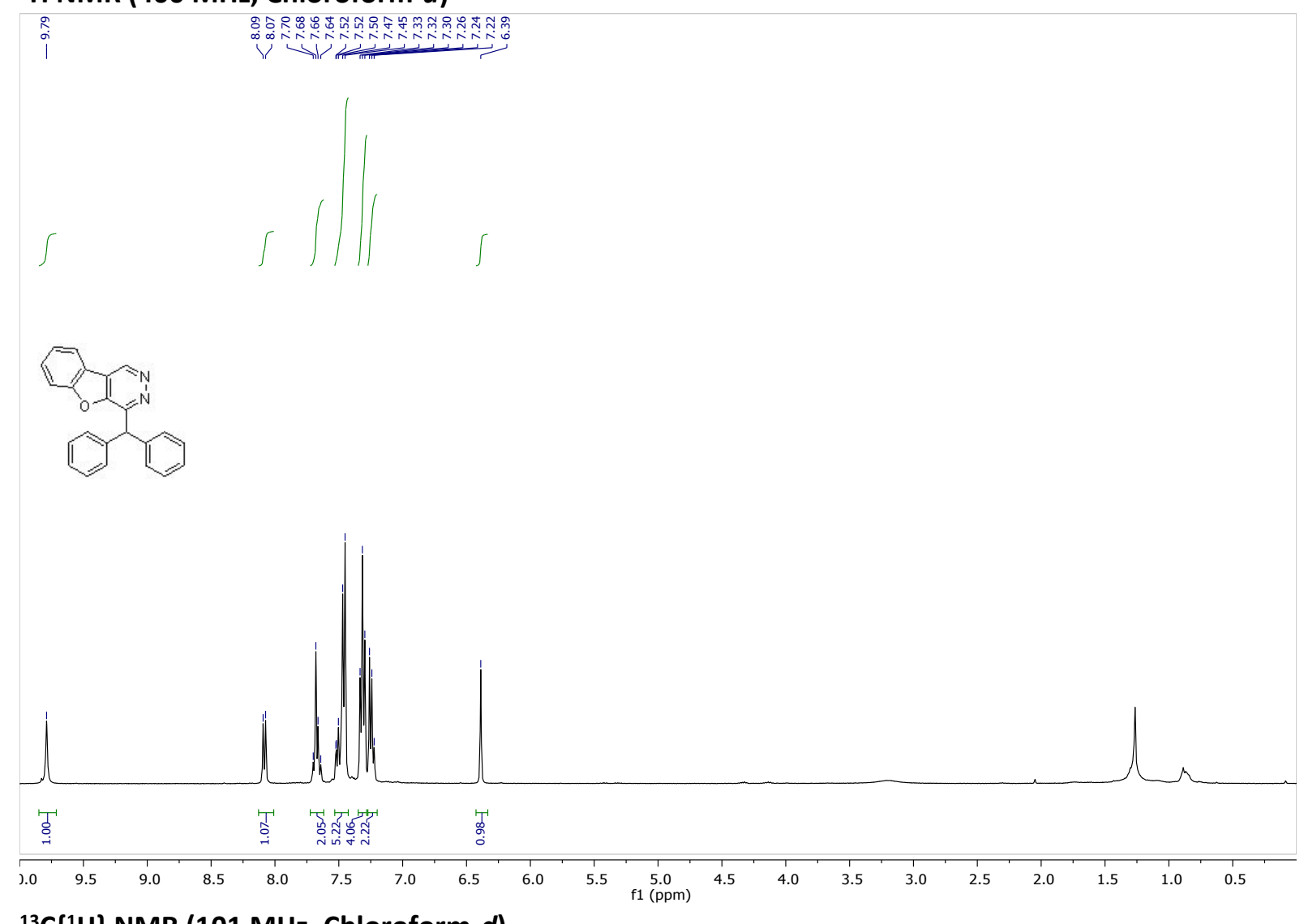

${ }^{13} \mathrm{C}\left\{{ }^{1} \mathrm{H}\right\}$ NMR (101 MHz, Chloroform-d)

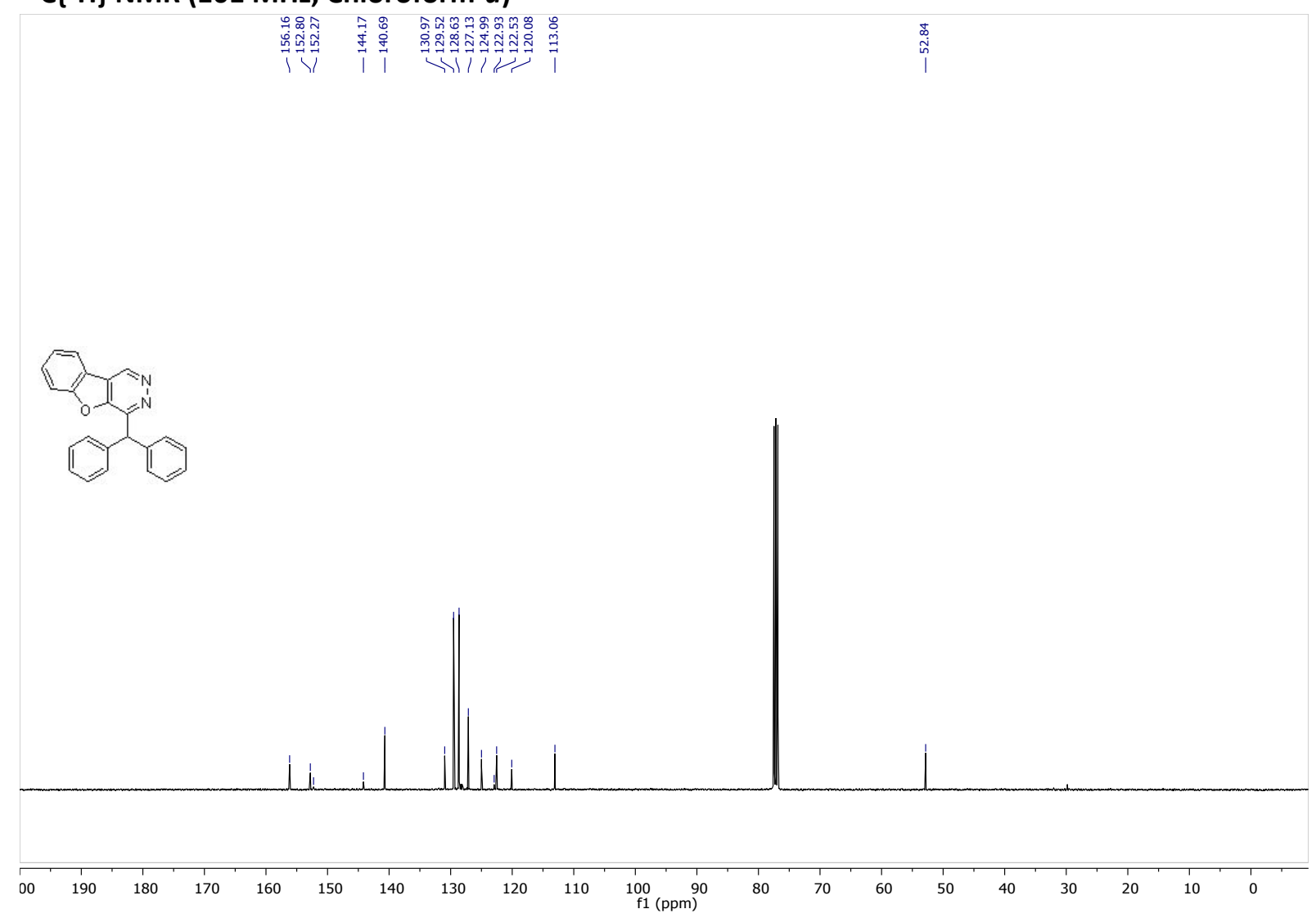

S75 
Compound 2p: 1-(phenyl(p-tolyl)methyl)phthalazine

${ }^{1} \mathrm{H}$ NMR (300 MHz, Chloroform-d)
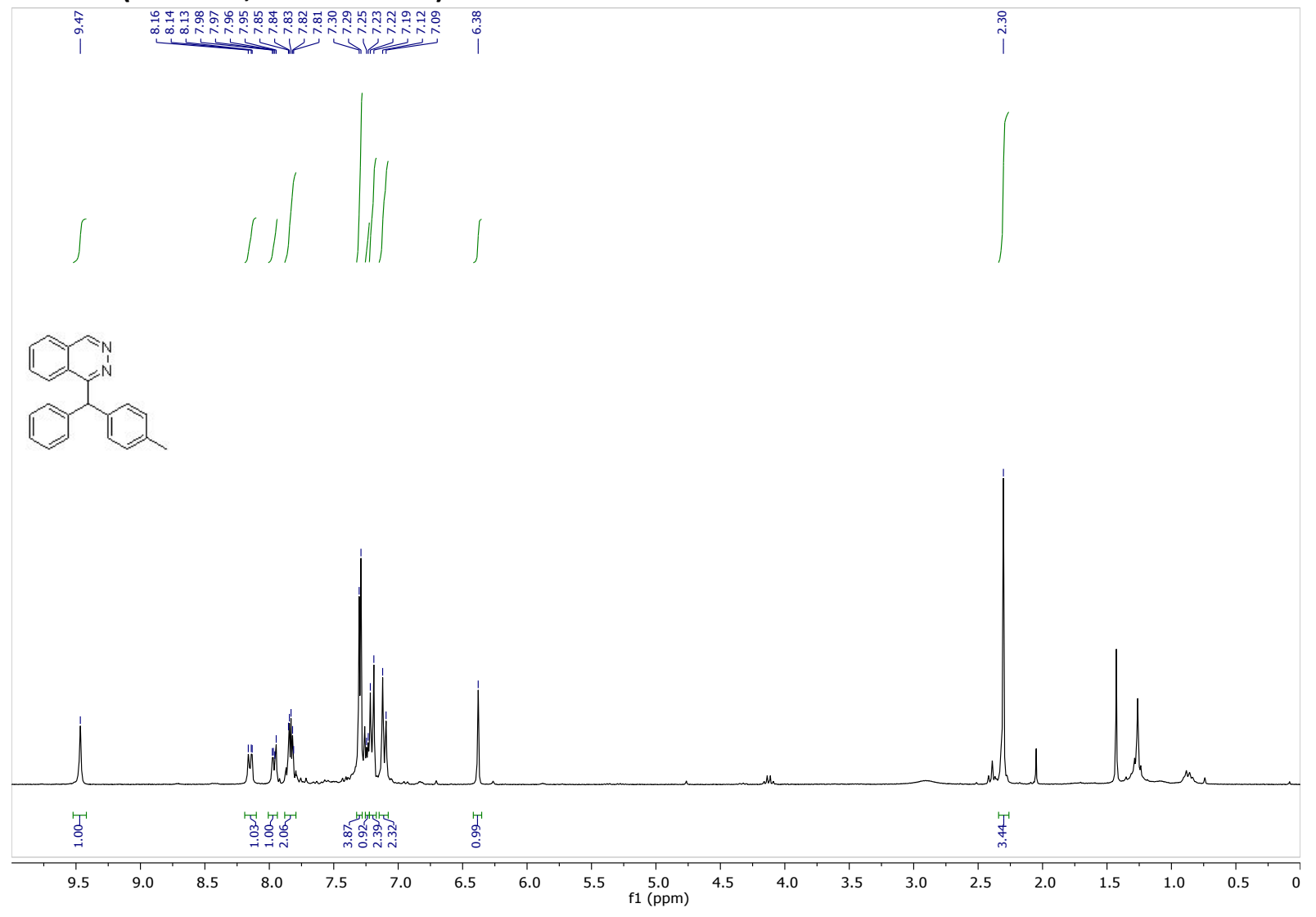

S76 


\section{Compound 2q: 1-(phenyl(o-tolyl)methyl)phthalazine}

${ }^{1} \mathrm{H}$ NMR (300 MHz, Chloroform-d)

象
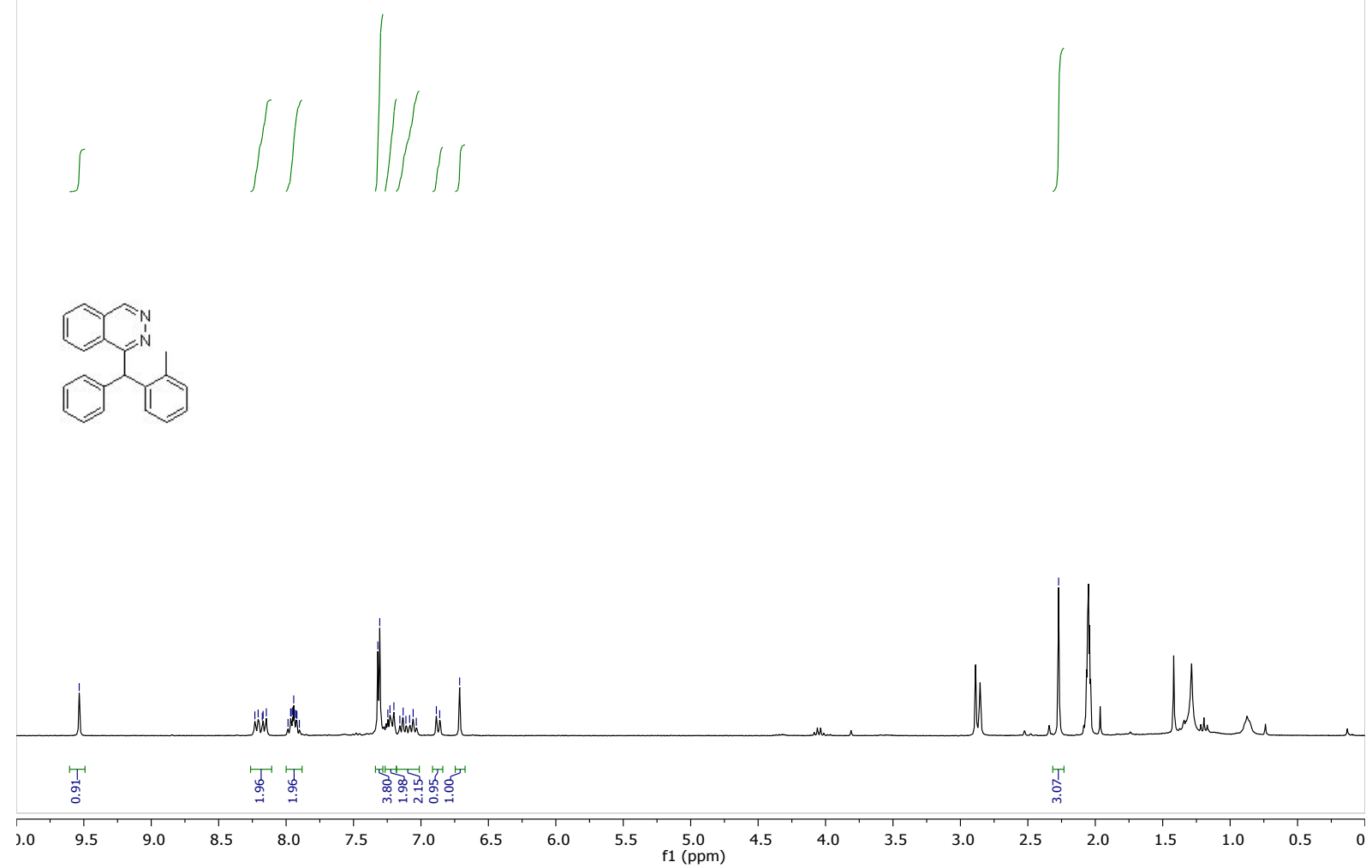

${ }^{13} \mathrm{C}\left\{{ }^{1} \mathrm{H}\right\}$ NMR (75 MHz, Chloroform-d)

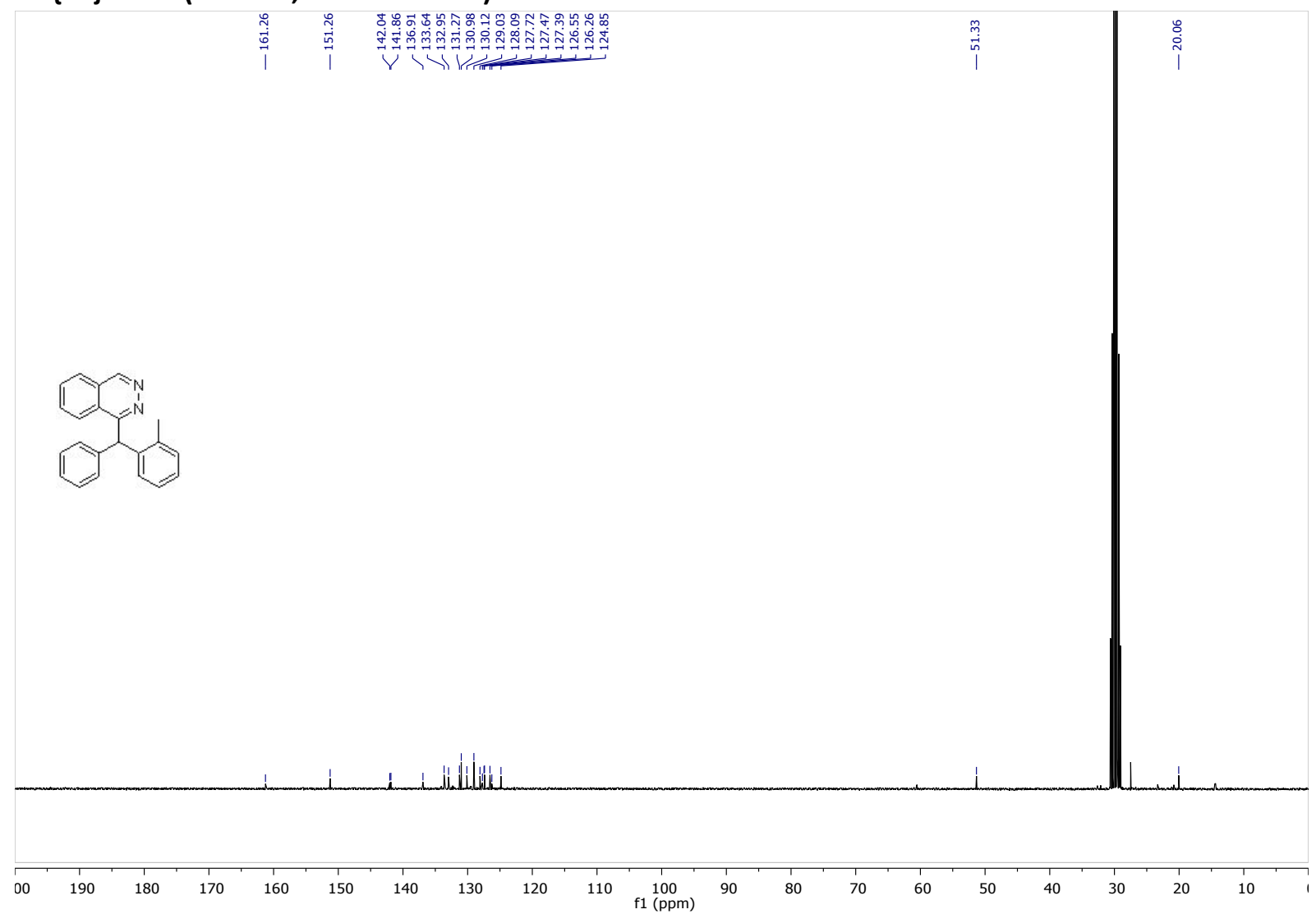




\section{Compound 2r: 1-(phenyl(3,4,5-trimethoxyphenyl)methyl)phthalazine}

${ }^{1} \mathrm{H}$ NMR (400 MHz, Chloroform-d)

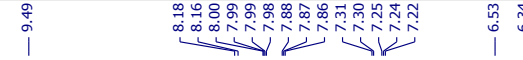
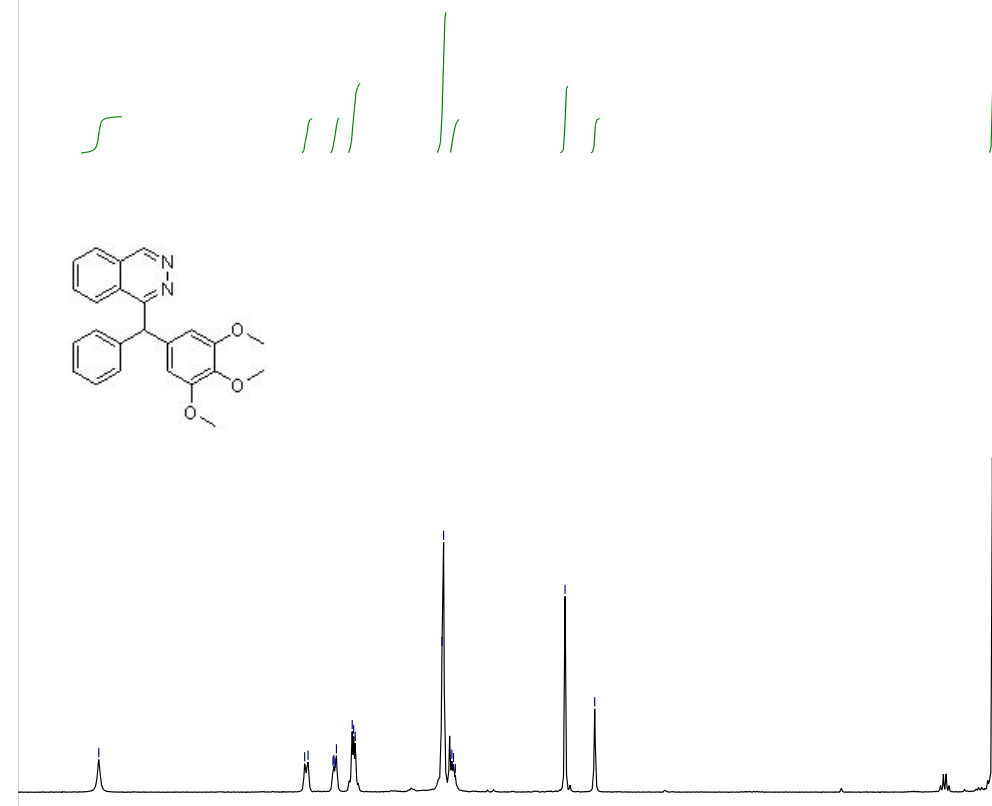

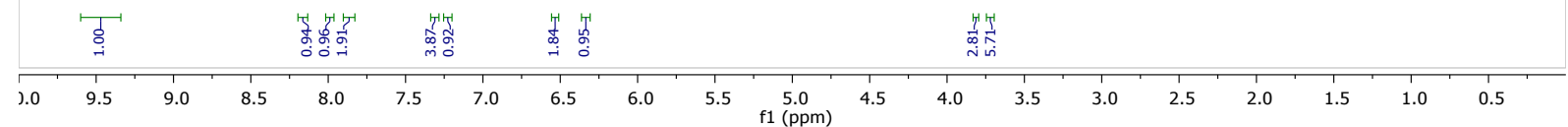




\section{Compound 2s: 1-(benzo[d][1,3]dioxol-5-yl(phenyl)methyl)phthalazine}

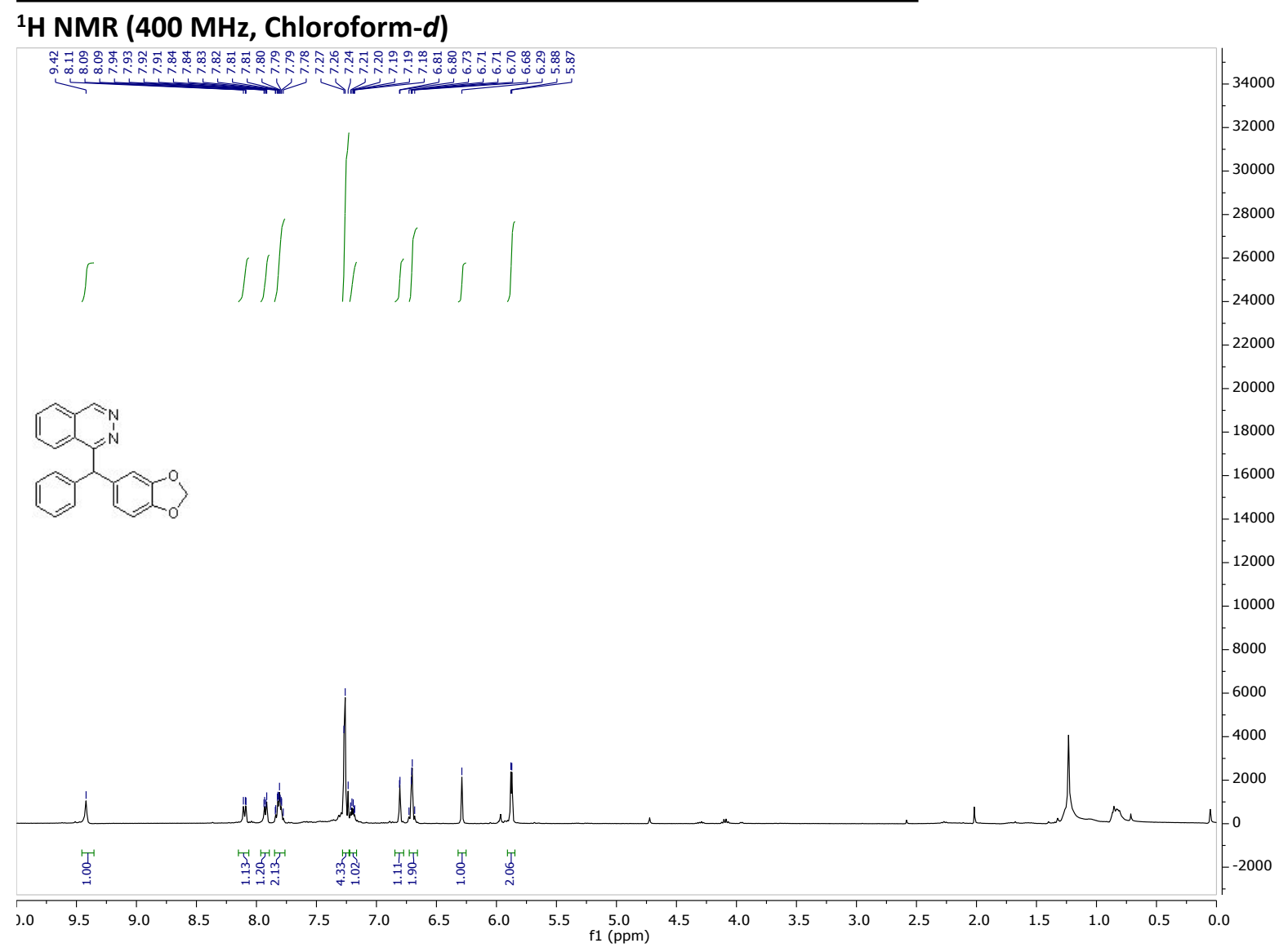

${ }^{13} \mathrm{C}\left\{{ }^{1} \mathrm{H}\right\}$ NMR (101 MHz, Chloroform-d)

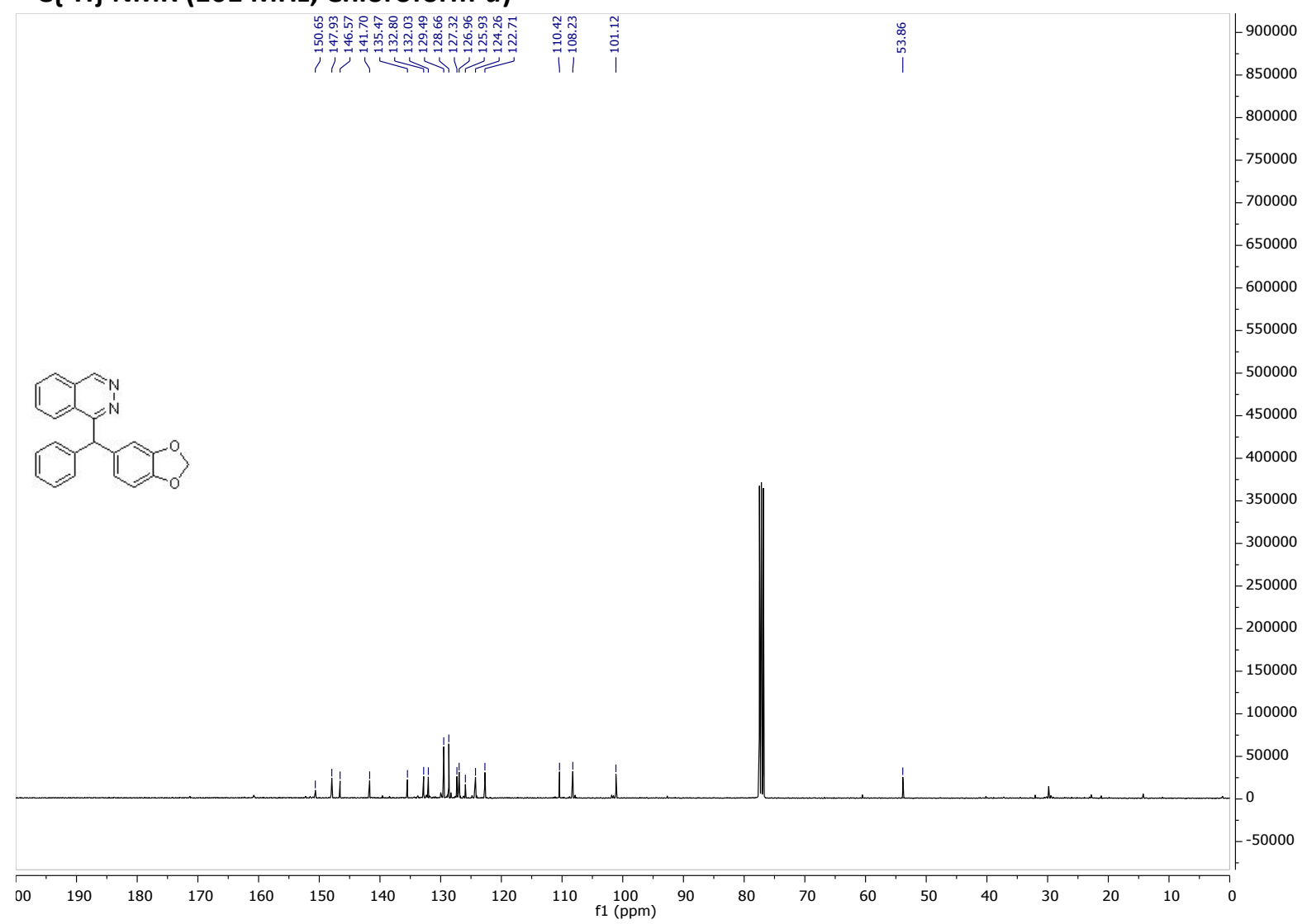


Compound 2t: 1-((4-fluorophenyl)(phenyl)methyl)phthalazine

${ }^{1} \mathrm{H}$ NMR (400 MHz, Chloroform-d)

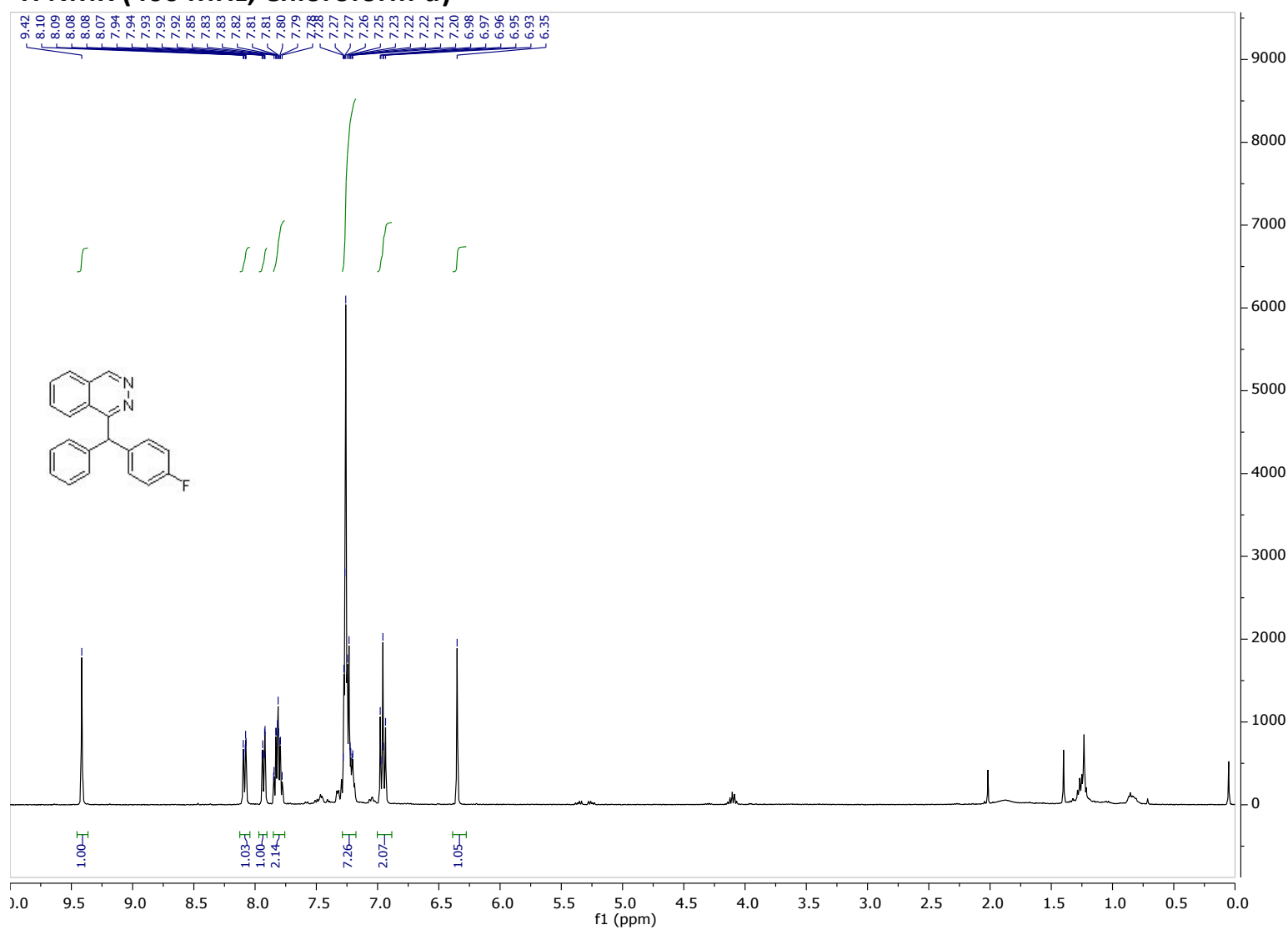

$\mathrm{S} 80$ 


$$
I
$$


Compound 3x: 1-(3-nitrobenzyl)phthalazine

${ }^{1} \mathrm{H}$ NMR (300 MHz, Chloroform-d)

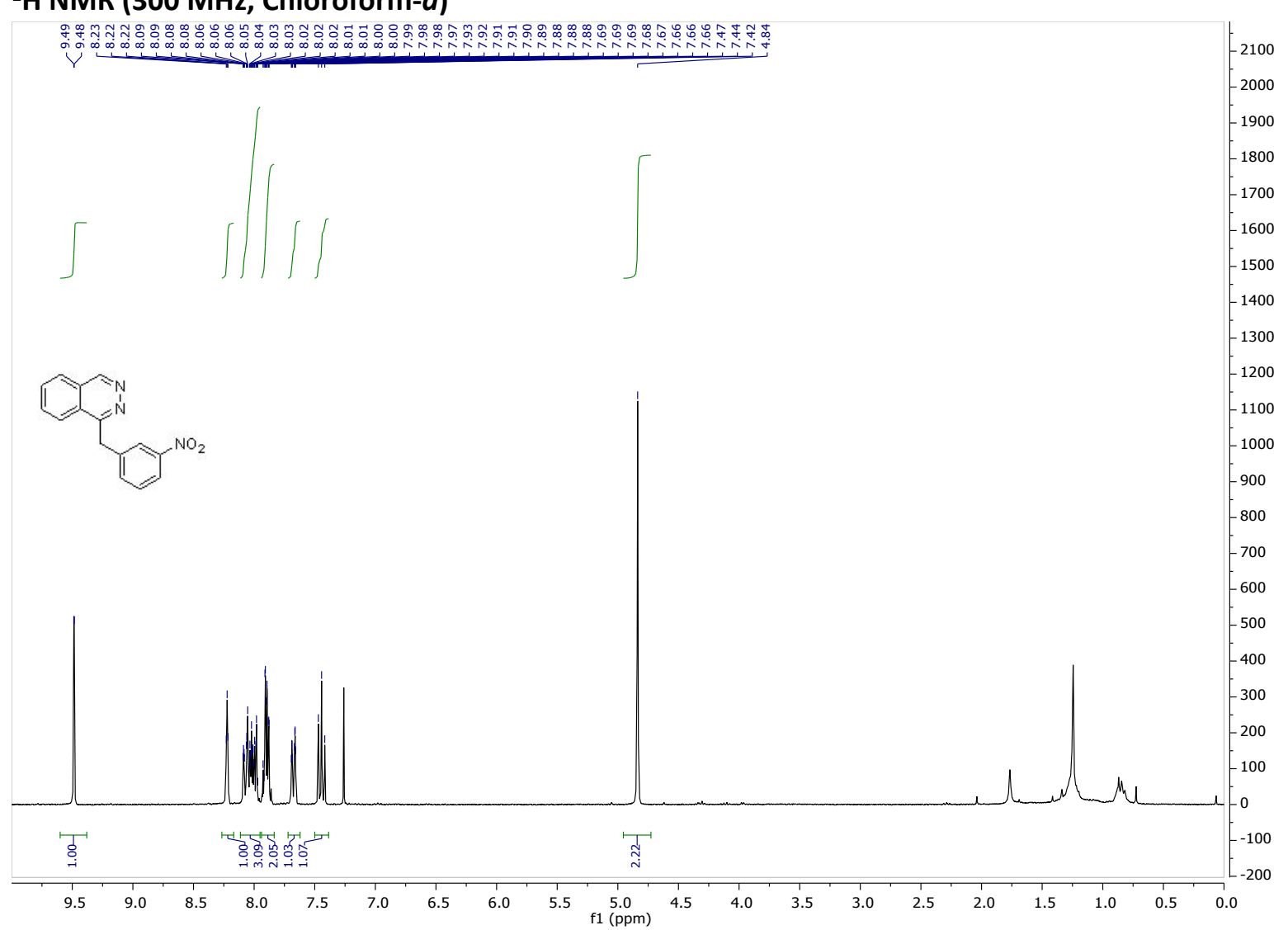

${ }^{13} \mathrm{C}\left\{{ }^{1} \mathrm{H}\right\}$ NMR (75 MHz, Chloroform- $d$ )

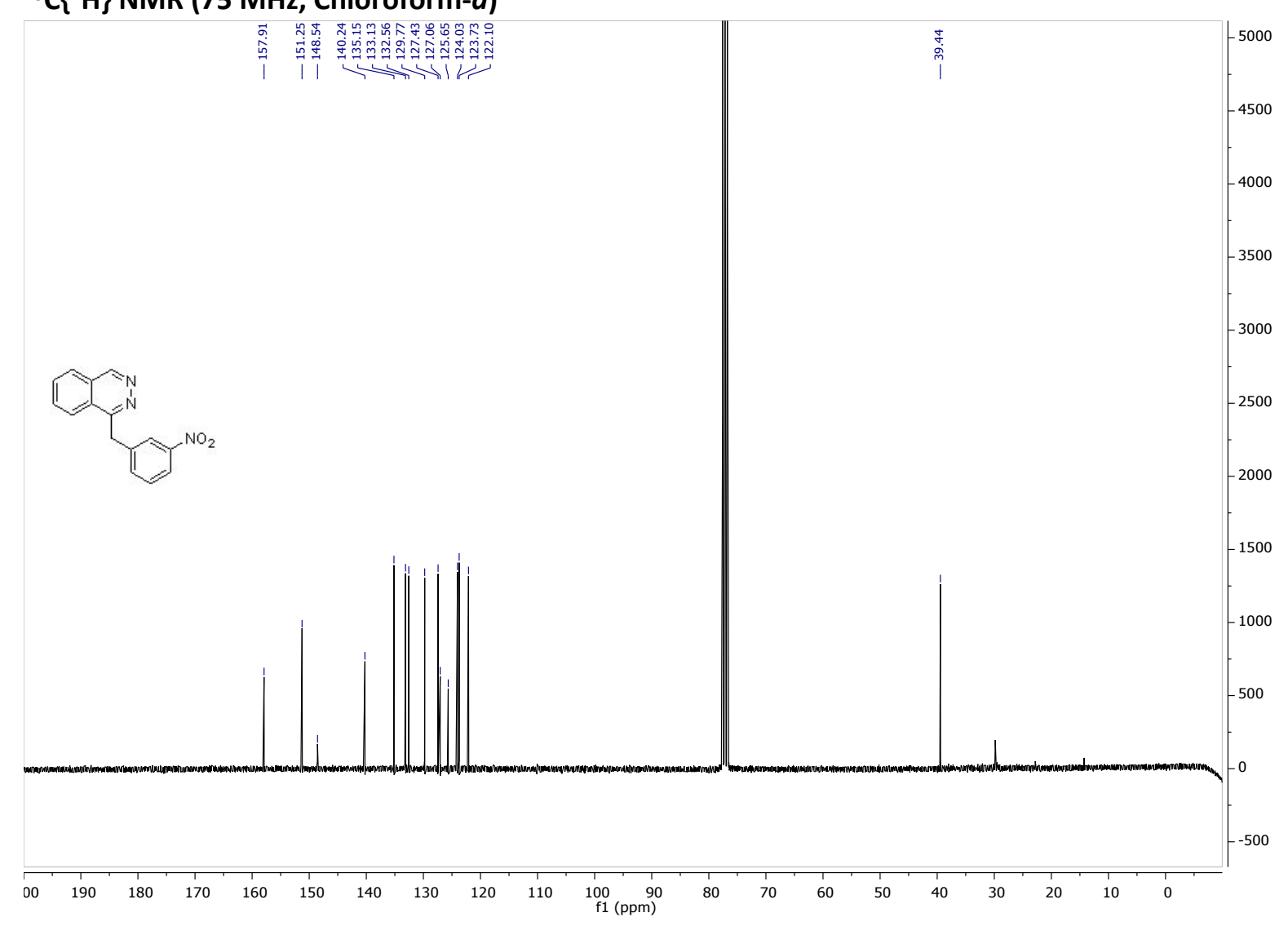

S82 
Compound 3y: 6-fluoro-1-(2-methoxyethyl)phthalazine

${ }^{1} \mathrm{H}$ NMR (300 MHz, Chloroform-d)

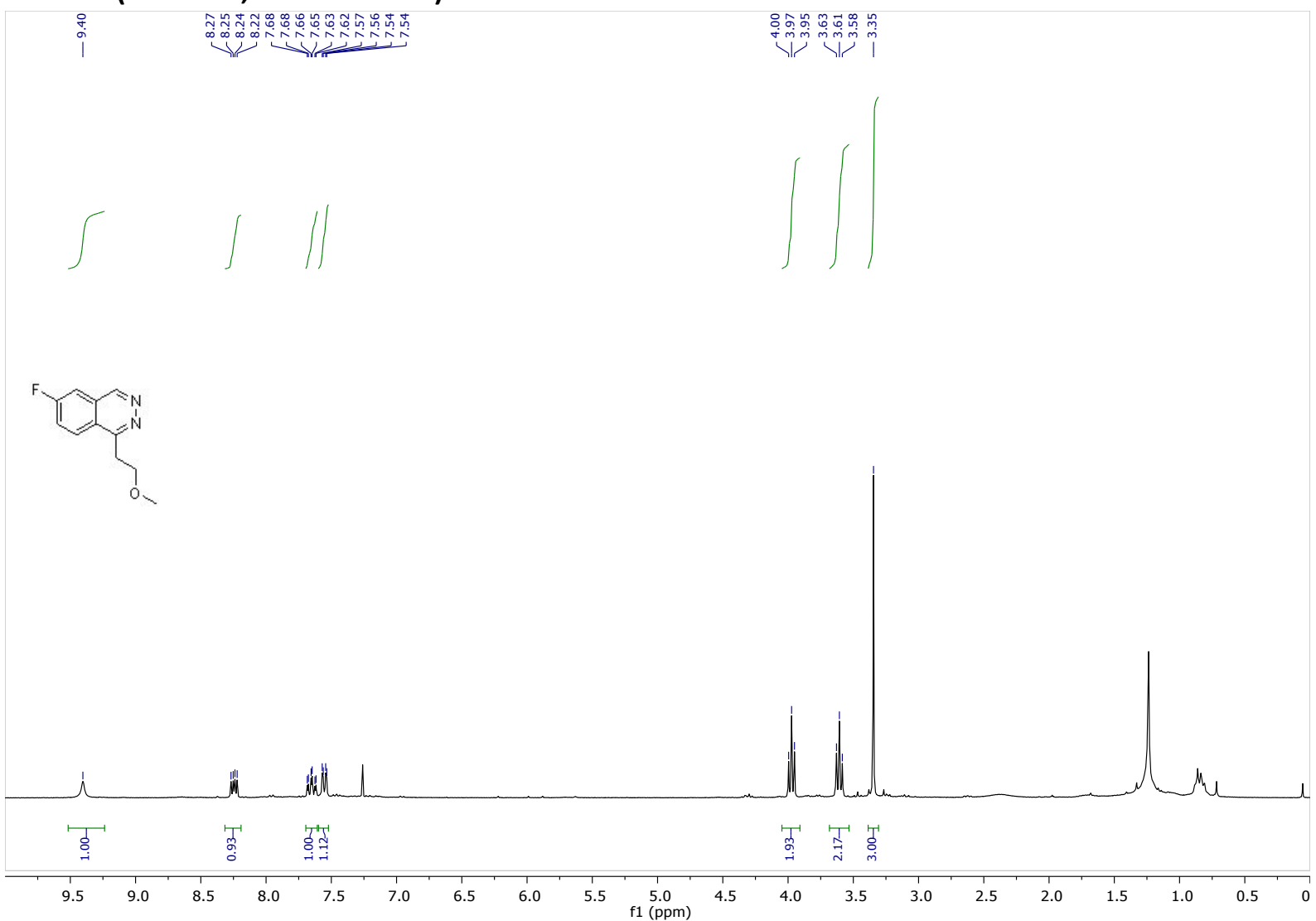

${ }^{13} \mathrm{C}\left\{{ }^{1} \mathrm{H}\right\}$ NMR (101 MHz, Chloroform-d)

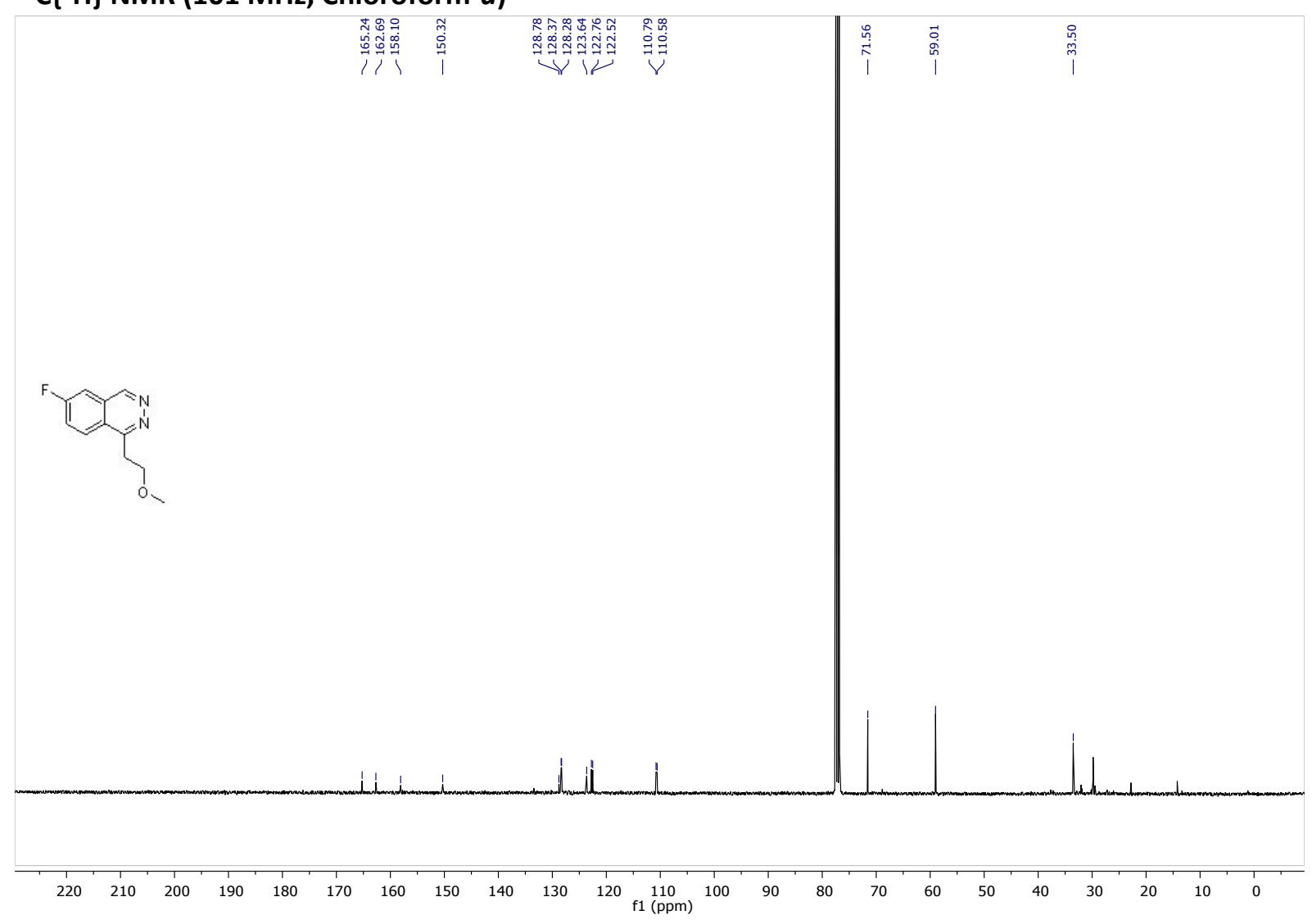


${ }^{19} \mathrm{~F}$ NMR (282 MHz, Chloroform-d)

i

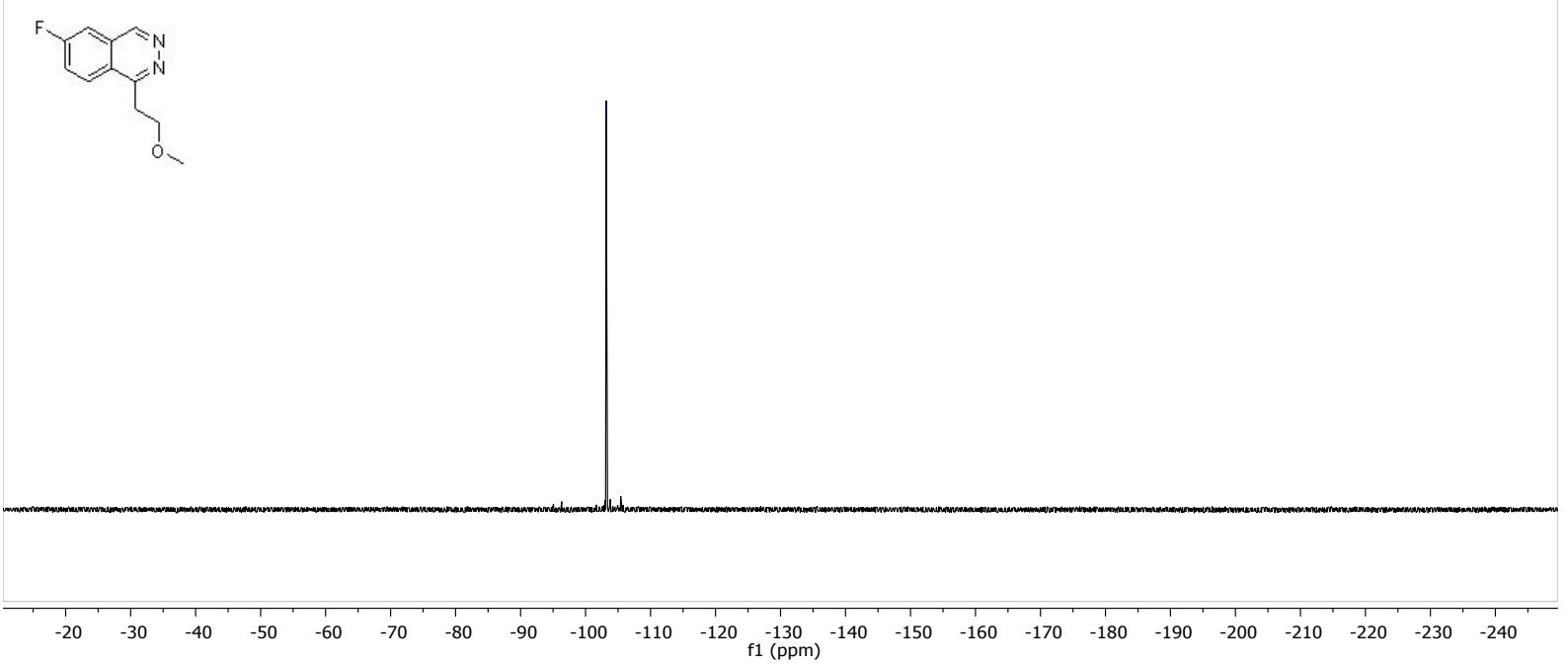

S84 


\section{VII- X-Ray structures}

For each crystal, we used the following protocol:

A suitable crystal was selected for structure elucidation on a Bruker VENTURE PHOTON100 ICMMO CMOS diffractometer. The crystal was kept at $273.15 \mathrm{~K}$ during data collection. Using Olex2 [1], the structure was solved with the olex2.solve [2] structure solution program using Charge Flipping and refined with the olex2.refine [3] refinement package using Gauss-Newton minimisation.

Compound $1 \mathrm{~h}, 2 \mathrm{a}$ and $2 \mathrm{j}$ were dissolved in hot ethyl acetate and allow to come back to room temperature leading to crystallization.

Compound 3a were dissolved in a mixture of hot acetone/MeOH (10/1) and allow to come back to room temperature leading to crystallization.

\section{Compound $1 \mathrm{~h}-\operatorname{CCDC} \mathrm{n}^{\circ} 2031023$ (ellipsoid contour $50 \%$ probability)}




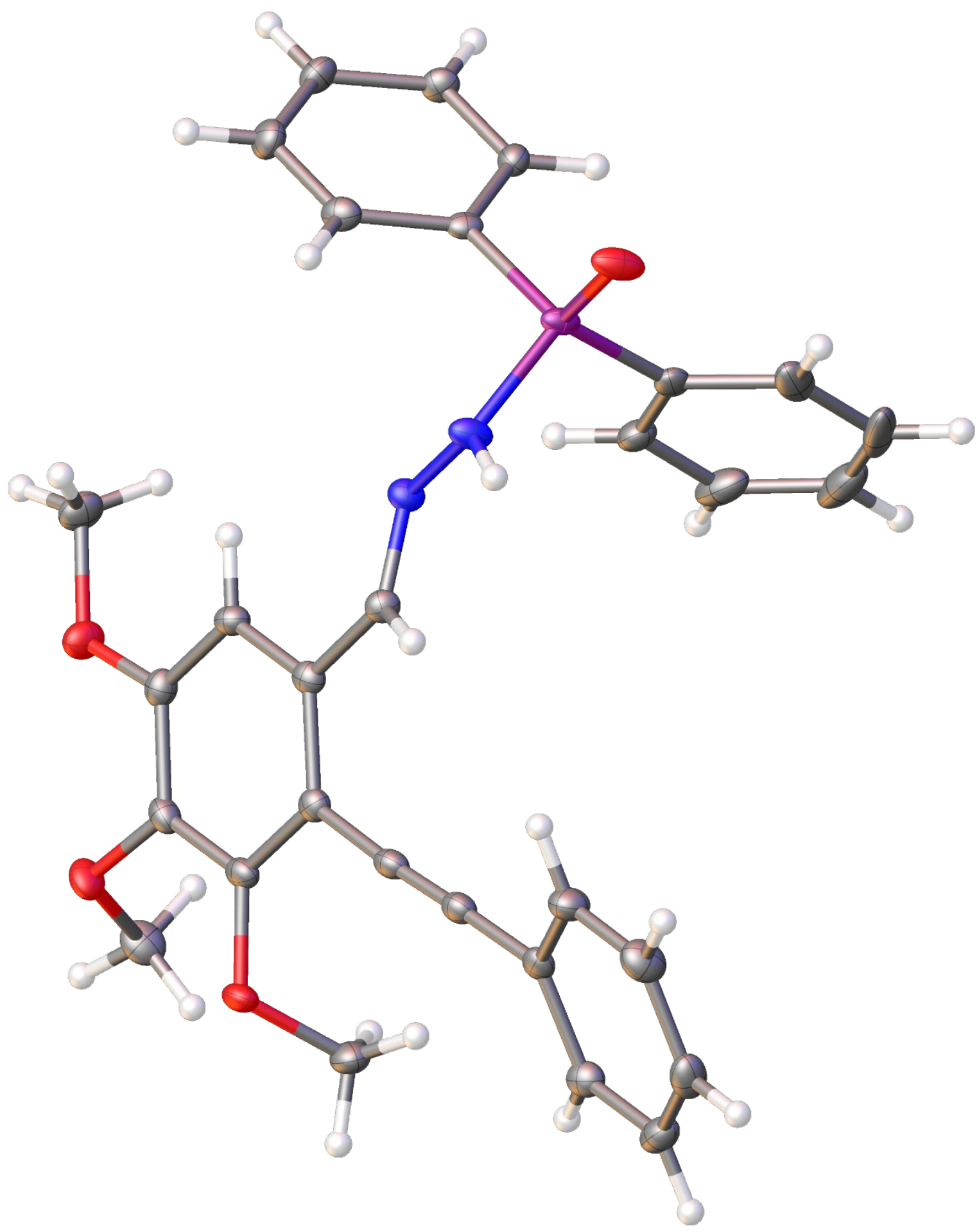




\begin{tabular}{|c|c|}
\hline \multicolumn{2}{|c|}{ Table S2 Crystal data and structure refinement for $\mathbf{1 h}$. } \\
\hline Identification code & mda467 \\
\hline Empirical formula & $\mathrm{C}_{30} \mathrm{H}_{27} \mathrm{~N}_{2} \mathrm{O}_{4} \mathrm{P}$ \\
\hline Formula weight & 510.50 \\
\hline Temperature/K & $273(2)$ \\
\hline Crystal system & triclinic \\
\hline Space group & P-1 \\
\hline$a / \AA$ & $11.0362(4)$ \\
\hline $\mathrm{b} / \AA ̊ \AA$ & $12.1195(5)$ \\
\hline$c / \AA ̊$ & $12.4369(5)$ \\
\hline$\alpha /^{\circ}$ & $101.1580(10)$ \\
\hline$\beta /^{\circ}$ & $108.9870(10)$ \\
\hline$\gamma /{ }^{\circ}$ & $116.5000(10)$ \\
\hline Volume $/ \AA^{3}$ & $1290.04(9)$ \\
\hline Z & 2 \\
\hline$\rho_{\text {calcg}} \mathrm{g} / \mathrm{cm}^{3}$ & 1.314 \\
\hline$\mu / \mathrm{mm}^{-1}$ & 1.265 \\
\hline $\mathrm{F}(000)$ & 536.0 \\
\hline Crystal size $/ \mathrm{mm}^{3}$ & $? \times ? \times ?$ \\
\hline Radiation & CuK $\alpha(\lambda=1.54178)$ \\
\hline $2 \Theta$ range for data collection ${ }^{\circ}$ & 8.204 to 133.398 \\
\hline Index ranges & $-13 \leq h \leq 13,-14 \leq \mathrm{k} \leq 14,-14 \leq \mathrm{I} \leq 14$ \\
\hline Reflections collected & 42161 \\
\hline Independent reflections & $4561\left[R_{\text {int }}=0.0268, R_{\text {sigma }}=0.0140\right]$ \\
\hline Data/restraints/parameters & $4561 / 0 / 341$ \\
\hline Goodness-of-fit on $F^{2}$ & 1.039 \\
\hline Final $R$ indexes $[I>=2 \sigma(I)]$ & $R_{1}=0.0315, w R_{2}=0.0817$ \\
\hline Final $R$ indexes [all data] & $R_{1}=0.0323, w R_{2}=0.0824$ \\
\hline Largest diff. peak/hole / e $\AA^{-3}$ & $0.25 /-0.41$ \\
\hline
\end{tabular}




\begin{tabular}{|c|c|c|c|c|}
\hline \multicolumn{5}{|c|}{$\begin{array}{l}\text { Table S3 Fractional Atomic Coordinates }\left(\times 10^{4}\right) \text { and Equivalent Isotropic Displacement Parameters } \\
\left(\AA^{2} \times 10^{3}\right) \text { for } 1 \mathbf{h} . U_{\text {eq }} \text { is defined as } 1 / 3 \text { of of the trace of the orthogonalised } U_{1} \text { tensor. }\end{array}$} \\
\hline Atom & $x$ & $y$ & $z$ & $\mathrm{U}(\mathrm{eq})$ \\
\hline $\mathrm{C} 1$ & 7738.4(15) & 2012.4(13) & $7796.6(12)$ & $17.5(3)$ \\
\hline $\mathrm{C} 2$ & $8319.8(19)$ & $1201.5(16)$ & 7853.5(13) & $28.0(3)$ \\
\hline $\mathrm{C3}$ & $9904(2)$ & $1806(2)$ & $8483.5(15)$ & 41.2(5) \\
\hline $\mathrm{C4}$ & $10886.8(19)$ & $3190(2)$ & 9027.5(15) & $39.2(4)$ \\
\hline C5 & $10318.6(16)$ & 3991.4(17) & 8959.5(14) & $30.5(3)$ \\
\hline $\mathrm{C6}$ & $8749.6(15)$ & $3410.6(14)$ & $8353.7(12)$ & $19.8(3)$ \\
\hline $\mathrm{C7}$ & $5232.8(13)$ & $2087.8(11)$ & 7951.3(11) & 13.1(2) \\
\hline $\mathrm{C} 8$ & $4063.7(14)$ & $2298.5(13)$ & $7456.6(12)$ & 17.6(3) \\
\hline C9 & $3698.7(15)$ & $2922.3(14)$ & $8235.5(12)$ & $20.4(3)$ \\
\hline $\mathrm{C} 10$ & $4495.2(14)$ & $3336.1(13)$ & 9511.1(12) & $18.2(3)$ \\
\hline $\mathrm{C} 11$ & $5646.4(14)$ & $3117.9(12)$ & $10008.9(11)$ & $16.5(3)$ \\
\hline $\mathrm{C} 12$ & $6016.7(14)$ & $2493.4(12)$ & $9231.3(11)$ & $14.9(2)$ \\
\hline $\mathrm{C} 13$ & $6341.8(14)$ & $3439.2(12)$ & $5194.4(11)$ & $14.6(2)$ \\
\hline C14 & 6979.0(13) & $4844.0(12)$ & $5346.6(11)$ & 14.2(2) \\
\hline $\mathrm{C} 15$ & $7746.6(13)$ & $5334.2(12)$ & $4670.3(11)$ & $14.5(3)$ \\
\hline C16 & $8341.7(14)$ & $6683.2(13)$ & 4814.4(11) & $15.3(3)$ \\
\hline $\mathrm{C} 17$ & $8147.7(14)$ & $7508.7(12)$ & 5591.4(12) & $16.3(3)$ \\
\hline $\mathrm{C} 18$ & 7383.6(14) & $7001.2(13)$ & 6264.1(11) & $16.3(3)$ \\
\hline C19 & $6807.8(13)$ & $5684.4(12)$ & 6139.6(11) & $15.2(3)$ \\
\hline $\mathrm{C} 20$ & $6575.2(16)$ & $7449.2(14)$ & $7762.3(13)$ & 23.1(3) \\
\hline $\mathrm{C} 21$ & $10232.0(16)$ & $9748.7(13)$ & $6327.3(13)$ & $23.7(3)$ \\
\hline $\mathrm{C} 22$ & 10506.1(15) & 7358.1(14) & 4442.4(13) & $21.3(3)$ \\
\hline $\mathrm{C} 23$ & $7896.6(14)$ & $4464.9(12)$ & $3840.7(11)$ & $15.7(3)$ \\
\hline $\mathrm{C} 24$ & $8003.5(14)$ & $3689.0(12)$ & $3172.2(11)$ & $16.3(3)$ \\
\hline $\mathrm{C} 25$ & $8151.8(14)$ & 2794.1(13) & 2365.9(11) & 15.3(3) \\
\hline $\mathrm{C} 26$ & 7078.0(15) & $1402.7(13)$ & $1835.8(12)$ & $20.7(3)$ \\
\hline $\mathrm{C} 27$ & $7218.5(17)$ & $559.6(14)$ & $1030.2(13)$ & $24.8(3)$ \\
\hline $\mathrm{C} 28$ & $8421.7(16)$ & $1082.8(14)$ & $749.4(12)$ & $23.0(3)$ \\
\hline $\mathrm{C} 29$ & 9498.2(15) & $2456.5(14)$ & $1281.6(12)$ & $21.7(3)$ \\
\hline $\mathrm{C} 30$ & 9372.9(14) & $3312.8(13)$ & 2088.2(11) & 18.0(3) \\
\hline N1 & $5356.7(12)$ & $1705.9(10)$ & $5752.4(10)$ & $16.9(2)$ \\
\hline N2 & $6042.5(12)$ & $3073.7(10)$ & $6025.2(9)$ & $15.2(2)$ \\
\hline 01 & $4834.5(11)$ & $-232.8(9)$ & $6480.9(9)$ & $23.3(2)$ \\
\hline $\mathrm{O} 2$ & 7271.6(11) & $7896.6(9)$ & $7013.9(9)$ & $21.3(2)$ \\
\hline $\mathrm{O3}$ & $8606.2(10)$ & $8789.0(9)$ & $5671.6(9)$ & $21.2(2)$ \\
\hline $\mathrm{O4}$ & $9036.4(10)$ & $7191.5(9)$ & $4131.8(8)$ & $17.9(2)$ \\
\hline $\mathrm{P}$ & $5702.8(4)$ & $1259.3(3)$ & $6947.6(3)$ & $14.48(10)$ \\
\hline
\end{tabular}




\begin{tabular}{|c|c|c|c|c|c|c|}
\hline \multicolumn{7}{|c|}{$\begin{array}{l}\text { Table S4 Anisotropic Displacement Parameters }\left(\AA^{2} \times 10^{3}\right) \text { for } \mathbf{1 h} \text {. The Anisotropic displacement } \\
\text { factor exponent takes the form: }-2 \pi^{2}\left[h^{2} a^{*} U_{11}+2 h k a * b * U_{12}+\ldots\right] .\end{array}$} \\
\hline Atom & $\mathrm{U}_{11}$ & $\mathbf{U}_{22}$ & $\mathbf{U}_{33}$ & $\mathbf{U}_{23}$ & $\mathrm{U}_{13}$ & $\mathrm{U}_{12}$ \\
\hline C1 & $24.2(7)$ & $24.1(7)$ & $16.2(6)$ & $11.4(5)$ & $15.2(5)$ & $16.7(6)$ \\
\hline $\mathrm{C} 2$ & 49.7(9) & $38.8(8)$ & $25.4(7)$ & $20.6(7)$ & $27.8(7)$ & $35.9(8)$ \\
\hline $\mathrm{C3}$ & $60.9(11)$ & $86.1(14)$ & $34.4(9)$ & $39.3(10)$ & $36.6(9)$ & $66.5(12)$ \\
\hline C4 & $29.3(8)$ & $77.0(13)$ & $27.6(8)$ & $24.0(9)$ & $18.8(7)$ & $36.1(9)$ \\
\hline $\mathrm{C5}$ & 19.5(7) & $46.4(9)$ & $23.4(7)$ & 13.6(7) & 13.0(6) & $14.5(7)$ \\
\hline $\mathrm{C6}$ & $18.7(6)$ & $25.3(7)$ & $20.7(7)$ & $11.0(6)$ & 13.0(5) & $12.6(6)$ \\
\hline C7 & 13.0(6) & $9.4(5)$ & $14.0(6)$ & $4.2(5)$ & $7.6(5)$ & $3.2(5)$ \\
\hline $\mathrm{C} 8$ & $14.8(6)$ & 19.7(6) & $13.3(6)$ & $6.0(5)$ & $5.3(5)$ & $6.9(5)$ \\
\hline C9 & 16.1(6) & $26.5(7)$ & $21.3(7)$ & $10.3(6)$ & $8.2(5)$ & 13.6(6) \\
\hline C10 & $17.8(6)$ & 19.2(6) & 19.1(6) & $5.2(5)$ & $10.5(5)$ & 10.9(5) \\
\hline $\mathrm{C} 11$ & 16.6(6) & 18.1(6) & $11.7(6)$ & $4.0(5)$ & $6.1(5)$ & $8.5(5)$ \\
\hline $\mathrm{C} 12$ & 13.9(6) & $15.7(6)$ & 16.5(6) & $7.1(5)$ & $7.7(5)$ & $8.5(5)$ \\
\hline $\mathrm{C} 13$ & $14.5(6)$ & 14.3(6) & $12.4(6)$ & $3.7(5)$ & $5.9(5)$ & $6.9(5)$ \\
\hline C14 & $12.2(6)$ & 15.1(6) & $12.0(6)$ & $5.2(5)$ & $3.7(5)$ & $6.4(5)$ \\
\hline C15 & $13.3(6)$ & 16.9(6) & $12.0(6)$ & $5.7(5)$ & $4.5(5)$ & $8.4(5)$ \\
\hline C16 & $13.5(6)$ & $18.9(6)$ & $13.7(6)$ & $9.3(5)$ & $5.7(5)$ & $8.4(5)$ \\
\hline C17 & 15.1(6) & 14.2(6) & $17.8(6)$ & $7.8(5)$ & $5.5(5)$ & $7.8(5)$ \\
\hline C18 & $15.2(6)$ & 17.0(6) & 15.1(6) & $4.5(5)$ & $5.4(5)$ & $9.5(5)$ \\
\hline C19 & 13.8(6) & 17.2(6) & 13.8(6) & $6.8(5)$ & $6.9(5)$ & $7.3(5)$ \\
\hline $\mathrm{C} 20$ & $26.0(7)$ & $23.3(7)$ & $22.1(7)$ & $6.2(6)$ & 14.9(6) & $13.7(6)$ \\
\hline $\mathrm{C} 21$ & $24.0(7)$ & $14.7(6)$ & $22.1(7)$ & $4.7(5)$ & $6.9(6)$ & $6.8(6)$ \\
\hline $\mathrm{C} 22$ & 19.2(6) & $25.4(7)$ & $24.8(7)$ & 14.1(6) & 13.9(6) & 12.1(6) \\
\hline $\mathrm{C} 23$ & $15.0(6)$ & $18.0(6)$ & 14.3(6) & $9.5(5)$ & $6.8(5)$ & $8.0(5)$ \\
\hline $\mathrm{C} 24$ & 16.2(6) & $18.5(6)$ & $14.8(6)$ & $9.5(5)$ & $7.1(5)$ & $9.0(5)$ \\
\hline $\mathrm{C} 25$ & $17.3(6)$ & 19.9(6) & $11.3(6)$ & $8.2(5)$ & $6.3(5)$ & $11.7(5)$ \\
\hline $\mathrm{C} 26$ & $22.1(7)$ & $20.3(7)$ & $23.1(7)$ & $11.7(6)$ & $13.7(6)$ & $10.8(6)$ \\
\hline $\mathrm{C} 27$ & $30.7(7)$ & $17.3(6)$ & $24.7(7)$ & $8.1(6)$ & 13.0(6) & $12.0(6)$ \\
\hline $\mathrm{C} 28$ & $31.5(7)$ & $27.7(7)$ & $17.6(6)$ & $8.5(6)$ & $11.9(6)$ & $21.9(6)$ \\
\hline C29 & 19.2(6) & $32.3(7)$ & $18.2(6)$ & $11.3(6)$ & $10.6(5)$ & $15.8(6)$ \\
\hline C30 & 15.0(6) & $20.0(6)$ & 14.9(6) & $6.7(5)$ & $5.2(5)$ & $7.9(5)$ \\
\hline N1 & $23.0(6)$ & $10.2(5)$ & 13.5(5) & $3.0(4)$ & $10.2(4)$ & $5.8(4)$ \\
\hline N2 & $15.7(5)$ & $11.3(5)$ & 15.1(5) & $4.4(4)$ & $6.8(4)$ & $5.4(4)$ \\
\hline 01 & $36.4(5)$ & $12.0(4)$ & $20.6(5)$ & $5.9(4)$ & $19.0(4)$ & $8.9(4)$ \\
\hline $\mathrm{O} 2$ & $26.2(5)$ & 17.1(4) & $24.5(5)$ & $7.0(4)$ & $15.7(4)$ & $12.6(4)$ \\
\hline $\mathrm{O3}$ & $20.4(5)$ & $14.4(4)$ & $29.8(5)$ & $11.7(4)$ & $11.0(4)$ & $9.7(4)$ \\
\hline 04 & $18.4(4)$ & $22.1(5)$ & $19.0(4)$ & $14.0(4)$ & $11.2(4)$ & $11.4(4)$ \\
\hline$P$ & $19.79(17)$ & $10.35(16)$ & $13.37(16)$ & $4.41(12)$ & $10.69(13)$ & $6.43(13)$ \\
\hline
\end{tabular}




\begin{tabular}{|c|c|c|c|c|c|}
\hline \multicolumn{6}{|c|}{ Table S5 Bond Lengths for $\mathbf{1 h}$. } \\
\hline Atom & Atom & Length/Å & Atom & Atom & Length/Å \\
\hline C1 & $\mathrm{C} 2$ & $1.3938(19)$ & C16 & $\mathrm{O} 4$ & $1.3758(15)$ \\
\hline $\mathrm{C} 1$ & $\mathrm{C} 6$ & $1.3971(19)$ & C16 & C17 & $1.3900(18)$ \\
\hline C1 & $P$ & $1.8024(13)$ & C17 & 03 & $1.3712(15)$ \\
\hline $\mathrm{C} 2$ & $\mathrm{C3}$ & $1.397(2)$ & C17 & C18 & $1.4088(18)$ \\
\hline $\mathrm{C3}$ & $\mathrm{C} 4$ & $1.381(3)$ & C18 & $\mathrm{O} 2$ & $1.3659(15)$ \\
\hline C4 & C5 & $1.373(2)$ & C18 & C19 & $1.3820(18)$ \\
\hline $\mathrm{C} 5$ & $\mathrm{C} 6$ & $1.385(2)$ & $\mathrm{C} 20$ & $\mathrm{O} 2$ & $1.4282(16)$ \\
\hline $\mathrm{C7}$ & C12 & $1.3950(17)$ & $\mathrm{C} 21$ & $\mathrm{O3}$ & $1.4337(16)$ \\
\hline C7 & $\mathrm{C} 8$ & $1.3952(18)$ & $\mathrm{C} 22$ & 04 & $9(15)$ \\
\hline $\mathrm{C7}$ & $P$ & $1.7990(12)$ & $\mathrm{C} 23$ & $\mathrm{C} 24$ & $1.2041(19)$ \\
\hline $\mathrm{C} 8$ & C9 & $1.3837(19)$ & $\mathrm{C} 24$ & $\mathrm{C} 25$ & $1.4328(18)$ \\
\hline C9 & C10 & $1.3901(19)$ & $\mathrm{C} 25$ & $\mathrm{C} 26$ & $1.4002(18)$ \\
\hline C10 & C11 & $1.3851(18)$ & $\mathrm{C} 25$ & $\mathrm{C} 30$ & $1.4005(18)$ \\
\hline C11 & C12 & $1.3874(18)$ & $\mathrm{C} 26$ & $\mathrm{C} 27$ & $1.385(2)$ \\
\hline C13 & $\mathrm{N} 2$ & $1.2813(16)$ & $\mathrm{C} 27$ & $\mathrm{C} 28$ & $1.387(2)$ \\
\hline $\mathrm{C} 13$ & C14 & $1.4661(17)$ & $\mathrm{C} 28$ & $\mathrm{C} 29$ & $1.385(2)$ \\
\hline C14 & C19 & $1.3964(18)$ & $\mathrm{C} 29$ & $\mathrm{C} 30$ & $1.3848(19)$ \\
\hline C14 & C15 & $1.4092(17)$ & N1 & $\mathrm{N} 2$ & $1.3897(14)$ \\
\hline C15 & C16 & $1.4100(17)$ & N1 & $\mathrm{P}$ & $1.6657(11)$ \\
\hline C15 & $\mathrm{C} 23$ & $1.4310(17)$ & 01 & $\mathrm{P}$ & $1.4869(9)$ \\
\hline
\end{tabular}




\begin{tabular}{|c|c|c|c|c|c|c|c|}
\hline \multicolumn{8}{|c|}{ Table S6 Bond Angles for $\mathbf{1 h}$. } \\
\hline Atom & Atom & Atom & Angle/ ${ }^{\circ}$ & Atom & Atom & Atom & Angle/ $^{\circ}$ \\
\hline $\mathrm{C} 2$ & C1 & C6 & 119.46(13) & $\mathrm{O} 3$ & C17 & C18 & $118.60(11)$ \\
\hline $\mathrm{C} 2$ & $\mathrm{C} 1$ & $P$ & $120.08(11)$ & C16 & C17 & C18 & $119.62(11)$ \\
\hline C6 & $\mathrm{C} 1$ & $P$ & $120.39(10)$ & $\mathrm{O} 2$ & C18 & C19 & $124.57(11)$ \\
\hline $\mathrm{C} 1$ & $\mathrm{C} 2$ & $\mathrm{C3}$ & 119.21(15) & $\mathrm{O} 2$ & C18 & C17 & 115.09(11) \\
\hline C4 & C3 & $\mathrm{C} 2$ & 120.52(14) & C19 & C18 & C17 & 120.34(11) \\
\hline C5 & C4 & C3 & $120.41(15)$ & C18 & C19 & C14 & $120.32(11)$ \\
\hline $\mathrm{C4}$ & $\mathrm{C5}$ & C6 & $119.89(16)$ & $\mathrm{C} 24$ & $\mathrm{C} 23$ & C15 & 176.64(13) \\
\hline $\mathrm{C5}$ & C6 & $\mathrm{C} 1$ & $120.49(13)$ & $\mathrm{C} 23$ & $\mathrm{C} 24$ & $\mathrm{C} 25$ & $178.41(13)$ \\
\hline $\mathrm{C} 12$ & C7 & $\mathrm{C} 8$ & 119.71(11) & $\mathrm{C} 26$ & $\mathrm{C} 25$ & C30 & $119.30(12)$ \\
\hline C12 & C7 & $P$ & 119.81(9) & $\mathrm{C} 26$ & $\mathrm{C} 25$ & $\mathrm{C} 24$ & 120.84(11) \\
\hline $\mathrm{C} 8$ & $\mathrm{C7}$ & $\mathrm{P}$ & $120.47(9)$ & C30 & $\mathrm{C} 25$ & $\mathrm{C} 24$ & $119.86(11)$ \\
\hline $\mathrm{C9}$ & $\mathrm{C} 8$ & C7 & $120.03(11)$ & $\mathrm{C} 27$ & $\mathrm{C} 26$ & $\mathrm{C} 25$ & $119.87(12)$ \\
\hline $\mathrm{C} 8$ & $\mathrm{C9}$ & C10 & $119.95(12)$ & $\mathrm{C} 26$ & $\mathrm{C} 27$ & $\mathrm{C} 28$ & $120.47(13)$ \\
\hline C11 & C10 & C9 & $120.39(12)$ & C29 & $\mathrm{C} 28$ & $\mathrm{C} 27$ & $119.98(12)$ \\
\hline C10 & C11 & C12 & $119.85(11)$ & $\mathrm{C} 30$ & C29 & $\mathrm{C} 28$ & $120.25(12)$ \\
\hline C11 & C12 & $\mathrm{C7}$ & $120.07(11)$ & C29 & C30 & $\mathrm{C} 25$ & $120.14(12)$ \\
\hline N2 & C13 & C14 & $120.07(11)$ & N2 & N1 & $\mathrm{P}$ & $117.05(8)$ \\
\hline C19 & C14 & C15 & $120.22(11)$ & C13 & $\mathrm{N} 2$ & N1 & $115.27(10)$ \\
\hline C19 & C14 & C13 & $120.39(11)$ & C18 & $\mathrm{O} 2$ & $\mathrm{C} 20$ & $117.13(10)$ \\
\hline C15 & C14 & $\mathrm{C} 13$ & 119.38(11) & C17 & $\mathrm{O3}$ & $\mathrm{C} 21$ & 114.95(10) \\
\hline C14 & C15 & C16 & 118.91(11) & C16 & O4 & $\mathrm{C} 22$ & 115.74(9) \\
\hline C14 & C15 & $\mathrm{C} 23$ & $120.16(11)$ & 01 & $P$ & N1 & $109.38(6)$ \\
\hline C16 & C15 & $\mathrm{C} 23$ & $120.92(11)$ & 01 & $P$ & C7 & $116.46(5)$ \\
\hline 04 & C16 & C17 & $118.17(11)$ & N1 & $\mathrm{P}$ & $\mathrm{C7}$ & $104.60(6)$ \\
\hline 04 & C16 & C15 & $121.12(11)$ & 01 & $\mathrm{P}$ & $\mathrm{C} 1$ & $111.62(6)$ \\
\hline C17 & C16 & $\mathrm{C} 15$ & $120.57(11)$ & $\mathrm{N} 1$ & $\mathrm{P}$ & $\mathrm{C} 1$ & $108.47(6)$ \\
\hline $\mathrm{O} 3$ & C17 & C16 & 121.69(11) & C7 & $\mathrm{P}$ & $\mathrm{C} 1$ & $105.86(6)$ \\
\hline
\end{tabular}




\begin{tabular}{|l|l|l|l|l|}
\hline \multicolumn{5}{|c|}{ Table S7 Hydrogen Atom Coordinates (Å $\left.\times 10^{4}\right)$ and Isotropic Displacement Parameters $\left(\AA^{2} \times 10^{3}\right)$ for } \\
\hline \multicolumn{1}{|c|}{ Atom } & \multicolumn{1}{|c|}{$\boldsymbol{y}$} & \multicolumn{1}{c|}{$\boldsymbol{z}$} & U(eq) \\
\hline H2 & 7660.83 & 269.39 & 7476.58 & 34 \\
\hline H3 & 10300.44 & 1271.62 & 8537.12 & 49 \\
\hline H4 & 11939.76 & 3581.06 & 9442.47 & 47 \\
\hline H5 & 10985.88 & 4922.78 & 9319.36 & 37 \\
\hline H00P & 8366.74 & 3955.55 & 8317.76 & 24 \\
\hline H8 & 3529.57 & 2019.56 & 6602.93 & 21 \\
\hline H9 & 2921.06 & 3064.68 & 7905.86 & 25 \\
\hline H10 & 4253.77 & 3761.9 & 10033.44 & 22 \\
\hline H11 & 6169.87 & 3389.37 & 10862.36 & 20 \\
\hline H12 & 6788.31 & 2344.96 & 9563.87 & 18 \\
\hline H13 & 6154.59 & 2812.65 & 4494.04 & 17 \\
\hline H19 & 6303.92 & 5356.41 & 6586.17 & 18 \\
\hline H20A & 5556.8 & 6664.59 & 7239.47 & 35 \\
\hline H20B & 6528.59 & 8149.72 & 8222.91 & 35 \\
\hline H20C & 7172.09 & 7232.43 & 8327.45 & 35 \\
\hline H21A & 10643.66 & 9721.93 & 7123.89 & 36 \\
\hline H21B & 10445.8 & 10633.88 & 6437.34 & 36 \\
\hline H21C & 10695.13 & 9532.83 & 5859.81 & 36 \\
\hline H22A & 11195.15 & 7929.04 & 5305.73 & 32 \\
\hline H22B & 10920.25 & 7760.23 & 3949.35 & 32 \\
\hline H22C & 10378.71 & 6495.63 & 4278.17 & 32 \\
\hline H26 & 6271.95 & 1045.41 & 2024.22 & 25 \\
\hline H27 & 6501.01 & -363.82 & 675.07 & 30 \\
\hline H28 & 8505.68 & 511.31 & 203.84 & 28 \\
\hline H29 & 10308.18 & 2805.23 & 1096.67 & 26 \\
\hline H30 & 10101.06 & 4234.16 & 2446.12 & 22 \\
\hline H1 & $5274(19)$ & $1244(17)$ & $5090(17)$ & $29(4)$ \\
\hline & & & & \\
\hline
\end{tabular}




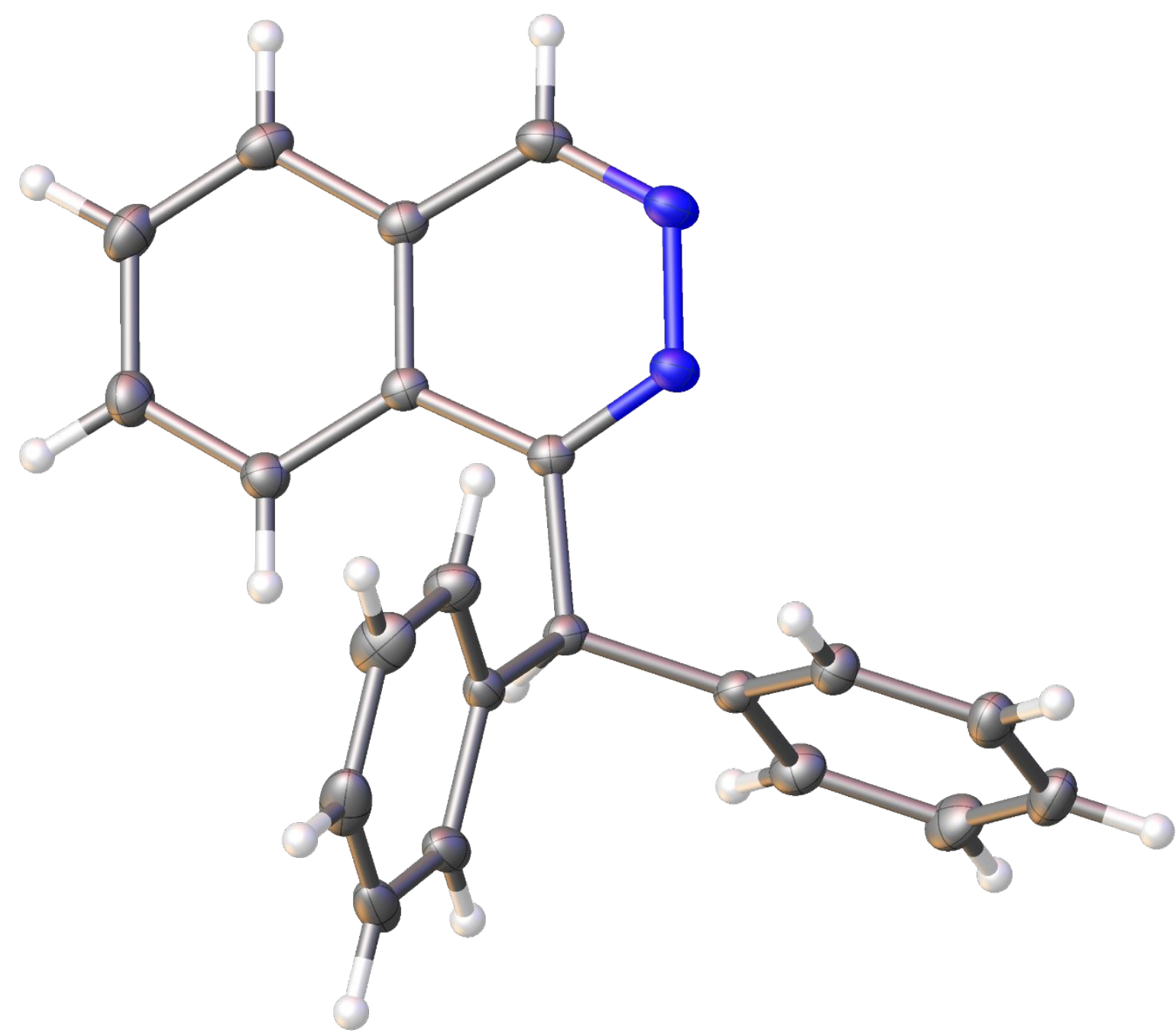




\begin{tabular}{|c|c|}
\hline \multicolumn{2}{|c|}{ Table S8 Crystal data and structure refinement for $2 a}$. \\
\hline Identification code & EB326 \\
\hline Empirical formula & $\mathrm{C}_{21} \mathrm{H}_{16} \mathrm{~N}_{2}$ \\
\hline Formula weight & 296.36 \\
\hline Temperature/K & $100(1)$ \\
\hline Crystal system & monoclinic \\
\hline Space group & $\mathrm{P} 2_{1} / \mathrm{n}$ \\
\hline $\mathrm{a} / \AA ̊$ & $8.8549(4)$ \\
\hline $\mathrm{b} / \AA ̊ \AA$ & $18.0318(9)$ \\
\hline$c / \AA ̊ A$ & $10.1285(5)$ \\
\hline$\alpha /^{\circ}$ & 90 \\
\hline$\beta /^{\circ}$ & $108.362(2)$ \\
\hline $\mathrm{V} /{ }^{\circ}$ & 90 \\
\hline Volume $/ \AA^{3}$ & $1534.87(13)$ \\
\hline Z & 4 \\
\hline$\rho_{\text {calcg }} \mathrm{g} / \mathrm{cm}^{3}$ & 1.282 \\
\hline$\mu / \mathrm{mm}^{-1}$ & 0.076 \\
\hline $\mathrm{F}(000)$ & 624.0 \\
\hline Crystal size $/ \mathrm{mm}^{3}$ & $0.110 \times 0.090 \times 0.060$ \\
\hline Radiation & $\operatorname{MoK} \alpha(\lambda=0.71073)$ \\
\hline $2 \Theta$ range for data collection $/^{\circ}$ & 4.518 to 61.064 \\
\hline Index ranges & $-12 \leq \mathrm{h} \leq 8,-25 \leq \mathrm{k} \leq 21,-14 \leq \mathrm{I} \leq 11$ \\
\hline Reflections collected & 21391 \\
\hline Independent reflections & $4628\left[R_{\text {int }}=0.0381, R_{\text {sigma }}=0.0285\right]$ \\
\hline Data/restraints/parameters & $4628 / 0 / 208$ \\
\hline Goodness-of-fit on $\mathrm{F}^{2}$ & 1.027 \\
\hline Final R indexes $[I>=2 \sigma(I)]$ & $\mathrm{R}_{1}=0.0468, w \mathrm{R}_{2}=0.1165$ \\
\hline Final $R$ indexes [all data] & $\mathrm{R}_{1}=0.0574, \mathrm{wR}_{2}=0.1245$ \\
\hline Largest diff. peak/hole / e $\AA^{-3}$ & $0.44 /-0.22$ \\
\hline
\end{tabular}




\begin{tabular}{|l|l|l|l|l|}
\hline \multicolumn{5}{|c|}{$\begin{array}{c}\text { Table S9 Fractional Atomic Coordinates }\left(\times 10^{4}\right) \text { and Equivalent Isotropic Displacement Parameters } \\
\left(\AA^{2} \times 10^{3}\right) \text { for 2a. } U_{\text {eq }} \text { is defined as } 1 / 3 \text { of of the trace of the orthogonalised } U_{\text {IJ }} \text { tensor. }\end{array}$} \\
\hline \multicolumn{1}{|c|}{ Atom } & \multicolumn{1}{|c|}{$\boldsymbol{x}$} & \multicolumn{1}{c|}{$\boldsymbol{y}$} & \multicolumn{1}{c|}{$\boldsymbol{Z}$} & \multicolumn{1}{c|}{ U(eq) } \\
\hline N2 & $6710.0(12)$ & $2076.0(6)$ & $5172.3(9)$ & $20.8(2)$ \\
\hline N1 & $6759.6(11)$ & $2420.4(5)$ & $3971.6(9)$ & $17.93(19)$ \\
\hline C2 & $4749.3(12)$ & $1584.2(6)$ & $2582.7(10)$ & $14.72(19)$ \\
\hline C1 & $5822.0(12)$ & $2201.2(6)$ & $2753.5(10)$ & $14.46(19)$ \\
\hline C9 & $5947.4(12)$ & $2618.9(6)$ & $1486.0(10)$ & $15.15(19)$ \\
\hline C7 & $4675.8(12)$ & $1245.6(6)$ & $3812.4(11)$ & $16.9(2)$ \\
\hline C10 & $6598.9(13)$ & $3401.1(6)$ & $1825.2(11)$ & $16.5(2)$ \\
\hline C3 & $3818.0(13)$ & $1295.0(6)$ & $1284.9(11)$ & $18.2(2)$ \\
\hline C8 & $5699.4(13)$ & $1535.5(6)$ & $5082.7(11)$ & $20.0(2)$ \\
\hline C16 & $6937.4(12)$ & $2191.4(6)$ & $752.3(10)$ & $16.0(2)$ \\
\hline C4 & $2888.4(13)$ & $680.1(6)$ & $1248.5(12)$ & $22.3(2)$ \\
\hline C21 & $6813.1(14)$ & $2374.7(7)$ & $-616.4(11)$ & $21.3(2)$ \\
\hline C6 & $3662.9(13)$ & $633.1(6)$ & $3754.5(12)$ & $21.3(2)$ \\
\hline C15 & $5592.2(14)$ & $4011.8(6)$ & $1469.6(12)$ & $21.7(2)$ \\
\hline C5 & $2804.0(14)$ & $350.0(7)$ & $2484.0(13)$ & $23.9(2)$ \\
\hline C11 & $8220.0(13)$ & $3518.9(6)$ & $2479.4(12)$ & $19.6(2)$ \\
\hline C12 & $8818.9(15)$ & $4230.7(7)$ & $2799.5(12)$ & $23.3(2)$ \\
\hline C17 & $8023.8(14)$ & $1647.7(6)$ & $1414.9(12)$ & $21.5(2)$ \\
\hline C13 & $7807.1(16)$ & $4837.8(7)$ & $2447.8(13)$ & $26.5(3)$ \\
\hline C19 & $8812.9(16)$ & $1475.9(7)$ & $-629.7(14)$ & $28.7(3)$ \\
\hline C20 & $7737.1(15)$ & $2017.0(7)$ & $-1304.8(12)$ & $26.0(3)$ \\
\hline C14 & $6196.9(16)$ & $4728.8(7)$ & $1777.4(14)$ & $27.0(3)$ \\
\hline C18 & $8954.0(16)$ & $1290.1(7)$ & $726.9(14)$ & $28.3(3)$ \\
\hline
\end{tabular}




\begin{tabular}{|l|l|l|l|l|l|l|}
\hline \multicolumn{7}{|c|}{ Table S10 Anisotropic Displacement Parameters $\left(\AA^{2} \times 10^{3}\right)$ for 2a. The Anisotropic displacement } \\
\hline \multicolumn{1}{|c|}{ Atom } & \multicolumn{1}{|c|}{$\mathbf{U}_{11}$} & \multicolumn{1}{c|}{$\mathbf{U}_{22}$} & \multicolumn{1}{c|}{$\mathbf{U}_{33}$} & \multicolumn{1}{c|}{$\mathbf{U}_{23}$} & \multicolumn{1}{|c|}{$\mathbf{U}_{13}$} & \multicolumn{1}{c|}{$\mathbf{U}_{12}$} \\
\hline N2 & $21.4(5)$ & $26.5(5)$ & $14.2(4)$ & $1.5(3)$ & $5.2(3)$ & $-2.2(4)$ \\
\hline N1 & $17.9(4)$ & $21.2(4)$ & $14.8(4)$ & $0.8(3)$ & $5.3(3)$ & $-1.7(3)$ \\
\hline C2 & $13.0(4)$ & $15.3(4)$ & $16.4(4)$ & $1.4(3)$ & $5.6(4)$ & $1.4(3)$ \\
\hline C1 & $13.4(4)$ & $15.5(4)$ & $15.1(4)$ & $1.3(3)$ & $5.3(4)$ & $1.5(3)$ \\
\hline C9 & $14.4(4)$ & $15.9(4)$ & $14.5(4)$ & $1.8(3)$ & $3.7(4)$ & $-1.0(3)$ \\
\hline C7 & $15.9(4)$ & $17.6(5)$ & $18.1(5)$ & $2.1(4)$ & $6.6(4)$ & $1.2(4)$ \\
\hline C10 & $19.2(5)$ & $15.6(4)$ & $16.0(4)$ & $1.4(3)$ & $7.3(4)$ & $-0.4(4)$ \\
\hline C3 & $17.1(5)$ & $19.6(5)$ & $17.4(5)$ & $0.5(4)$ & $4.6(4)$ & $-0.9(4)$ \\
\hline C8 & $20.7(5)$ & $24.1(5)$ & $16.3(5)$ & $3.2(4)$ & $7.3(4)$ & $0.0(4)$ \\
\hline C16 & $16.7(5)$ & $17.4(5)$ & $14.6(4)$ & $-1.6(3)$ & $5.8(4)$ & $-4.8(4)$ \\
\hline C4 & $19.3(5)$ & $22.8(5)$ & $22.7(5)$ & $-2.2(4)$ & $3.7(4)$ & $-3.7(4)$ \\
\hline C21 & $22.7(5)$ & $25.0(5)$ & $15.2(5)$ & $-0.3(4)$ & $4.4(4)$ & $-8.3(4)$ \\
\hline C6 & $20.3(5)$ & $21.5(5)$ & $23.5(5)$ & $4.9(4)$ & $9.1(4)$ & $-1.1(4)$ \\
\hline C15 & $22.2(5)$ & $19.9(5)$ & $23.5(5)$ & $2.8(4)$ & $8.1(4)$ & $3.5(4)$ \\
\hline C5 & $20.5(5)$ & $20.2(5)$ & $30.9(6)$ & $1.6(4)$ & $8.0(5)$ & $-4.8(4)$ \\
\hline C11 & $19.3(5)$ & $18.6(5)$ & $21.5(5)$ & $-0.1(4)$ & $7.0(4)$ & $-1.5(4)$ \\
\hline C12 & $24.6(5)$ & $22.0(5)$ & $24.7(5)$ & $-2.9(4)$ & $10.0(4)$ & $-6.8(4)$ \\
\hline C17 & $23.7(5)$ & $21.7(5)$ & $22.2(5)$ & $1.9(4)$ & $11.8(4)$ & $1.5(4)$ \\
\hline C13 & $37.2(7)$ & $17.1(5)$ & $29.8(6)$ & $-2.8(4)$ & $17.0(5)$ & $-5.5(5)$ \\
\hline C19 & $32.8(6)$ & $28.0(6)$ & $33.7(6)$ & $-10.4(5)$ & $22.5(5)$ & $-9.7(5)$ \\
\hline C20 & $30.2(6)$ & $32.7(6)$ & $18.0(5)$ & $-7.4(4)$ & $11.6(5)$ & $-16.5(5)$ \\
\hline C14 & $35.0(7)$ & $17.4(5)$ & $31.4(6)$ & $2.8(4)$ & $14.4(5)$ & $4.3(5)$ \\
\hline C18 & $29.8(6)$ & $25.4(6)$ & $36.5(7)$ & $0.6(5)$ & $20.2(5)$ & $3.9(5)$ \\
\hline
\end{tabular}

\begin{tabular}{|c|c|c|c|c|c|}
\hline \multicolumn{6}{|c|}{ Table S11 Bond Lengths for $\mathbf{2 a}$. } \\
\hline Atom & Atom & Length/Å & Atom & Atom & Length/Å \\
\hline N2 & $\mathrm{C} 8$ & $1.3067(15)$ & C3 & C4 & $1.3745(15)$ \\
\hline N2 & N1 & $1.3785(12)$ & C16 & C17 & $1.3893(15)$ \\
\hline N1 & $\mathrm{C} 1$ & $1.3123(13)$ & C16 & $\mathrm{C} 21$ & $1.3955(14)$ \\
\hline $\mathrm{C} 2$ & C7 & $1.4068(14)$ & C4 & C5 & $1.4089(17)$ \\
\hline $\mathrm{C} 2$ & C3 & $4130(14)$ & $\mathrm{C} 21$ & $\mathrm{C} 20$ & $5(17)$ \\
\hline $\mathrm{C} 2$ & $\mathrm{C} 1$ & $1.4376(14)$ & $\mathrm{C6}$ & C5 & $1.3704(17)$ \\
\hline $\mathrm{C} 1$ & C9 & $1.5224(14)$ & $\mathrm{C} 15$ & C14 & $1.3965(16)$ \\
\hline C9 & C10 & $5(14)$ & C11 & C12 & $1.3876(15)$ \\
\hline C9 & C16 & $1.5249(14)$ & $\mathrm{C} 12$ & $\mathrm{C} 13$ & $1.3881(17)$ \\
\hline $\mathrm{C7}$ & C6 & $1.4122(15)$ & C17 & $\mathrm{C} 18$ & $1.3934(16)$ \\
\hline C7 & $\mathrm{C} 8$ & $1.4197(15)$ & $\mathrm{C} 13$ & C14 & $1.3866(19)$ \\
\hline C10 & C15 & $1.3909(15)$ & C19 & $\mathrm{C} 18$ & $1.3812(19)$ \\
\hline C10 & C11 & $1.3949(15)$ & C19 & $\mathrm{C} 20$ & $1.384(2)$ \\
\hline
\end{tabular}




\begin{tabular}{|c|c|c|c|c|c|c|c|}
\hline \multicolumn{8}{|c|}{ Table S12 Bond Angles for $\mathbf{2 a}$. } \\
\hline Atom & Atom & Atom & Angle $/^{\circ}$ & Atom & Atom & Atom & Angle/ ${ }^{\circ}$ \\
\hline $\mathrm{C} 8$ & N2 & N1 & 119.09(9) & N2 & $\mathrm{C} 8$ & C7 & 124.43(10) \\
\hline $\mathrm{C} 1$ & N1 & N2 & $120.47(9)$ & C17 & C16 & C21 & $118.23(10)$ \\
\hline $\mathrm{C7}$ & $\mathrm{C} 2$ & $\mathrm{C} 3$ & $119.16(9)$ & C17 & C16 & C9 & $122.56(9)$ \\
\hline $\mathrm{C7}$ & $\mathrm{C} 2$ & $\mathrm{C} 1$ & $116.26(9)$ & $\mathrm{C} 21$ & C16 & C9 & 119.13(10) \\
\hline $\mathrm{C3}$ & $\mathrm{C} 2$ & $\mathrm{C} 1$ & $124.56(9)$ & $\mathrm{C} 3$ & $\mathrm{C} 4$ & $\mathrm{C5}$ & $121.05(11)$ \\
\hline N1 & $\mathrm{C} 1$ & $\mathrm{C} 2$ & $123.00(9)$ & $\mathrm{C} 20$ & $\mathrm{C} 21$ & C16 & $120.92(11)$ \\
\hline N1 & $\mathrm{C} 1$ & C9 & $116.82(9)$ & $\mathrm{C} 5$ & $\mathrm{C} 6$ & $\mathrm{C7}$ & 119.18(10) \\
\hline $\mathrm{C} 2$ & $\mathrm{C} 1$ & C9 & $120.16(9)$ & C10 & C15 & C14 & $120.38(11)$ \\
\hline $\mathrm{C} 10$ & $\mathrm{C} 9$ & $\mathrm{C} 1$ & $113.04(8)$ & C6 & $\mathrm{C} 5$ & $\mathrm{C} 4$ & 120.51(10) \\
\hline C10 & $\mathrm{C9}$ & C16 & $110.09(8)$ & $\mathrm{C} 12$ & C11 & C10 & $120.81(10)$ \\
\hline C1 & C9 & C16 & $111.51(8)$ & C11 & $\mathrm{C} 12$ & C13 & $120.08(11)$ \\
\hline $\mathrm{C} 2$ & $\mathrm{C7}$ & $\mathrm{C} 6$ & $120.56(10)$ & C16 & $\mathrm{C} 17$ & $\mathrm{C} 18$ & $120.89(11)$ \\
\hline $\mathrm{C} 2$ & $\mathrm{C} 7$ & $\mathrm{C} 8$ & $116.62(9)$ & C14 & $\mathrm{C} 13$ & $\mathrm{C} 12$ & $119.66(11)$ \\
\hline $\mathrm{C} 6$ & $\mathrm{C7}$ & $\mathrm{C} 8$ & $122.78(10)$ & $\mathrm{C} 18$ & C19 & $\mathrm{C} 20$ & $119.66(11)$ \\
\hline C15 & C10 & C11 & $118.82(10)$ & C19 & $\mathrm{C} 20$ & $\mathrm{C} 21$ & $120.10(11)$ \\
\hline C15 & C10 & C9 & $120.59(10)$ & $\mathrm{C} 13$ & C14 & C15 & $120.23(11)$ \\
\hline C11 & C10 & C9 & 120.58(9) & C19 & C18 & C17 & $120.18(12)$ \\
\hline C4 & C3 & C2 & $119.45(10)$ & & & & \\
\hline
\end{tabular}

\begin{tabular}{|l|l|l|l|l|}
\hline \multicolumn{5}{|c|}{ Table S13 Hydrogen Atom Coordinates $\left(\AA \times 10^{4}\right)$ and Isotropic Displacement Parameters $\left(\AA^{2} \times 10^{3}\right)$ for } \\
\hline \multicolumn{1}{|c|}{ Atom } & \multicolumn{1}{|c|}{$\boldsymbol{y}$} & \multicolumn{1}{c|}{$\boldsymbol{z}$} & U(eq) \\
\hline H9 & 4869 & 2663 & 830 & 18 \\
\hline H3 & 3834 & 1519 & 462 & 22 \\
\hline H8 & 5647 & 1327 & 5907 & 24 \\
\hline H4 & 2306 & 479 & 395 & 27 \\
\hline H21 & 6102 & 2741 & -1074 & 26 \\
\hline H6 & 3580 & 424 & 4568 & 26 \\
\hline H15 & 4510 & 3942 & 1024 & 26 \\
\hline H5 & 2161 & -63 & 2437 & 29 \\
\hline H11 & 8908 & 3115 & 2704 & 24 \\
\hline H12 & 9899 & 4301 & 3250 & 28 \\
\hline H17 & 8132 & 1521 & 2330 & 26 \\
\hline H13 & 8207 & 5315 & 2661 & 32 \\
\hline H19 & 9437 & 1239 & -1087 & 34 \\
\hline H20 & 7633 & 2141 & -2221 & 31 \\
\hline H14 & 5517 & 5135 & 1532 & 32 \\
\hline H18 & 9672 & 925 & 1182 & 34 \\
\hline
\end{tabular}




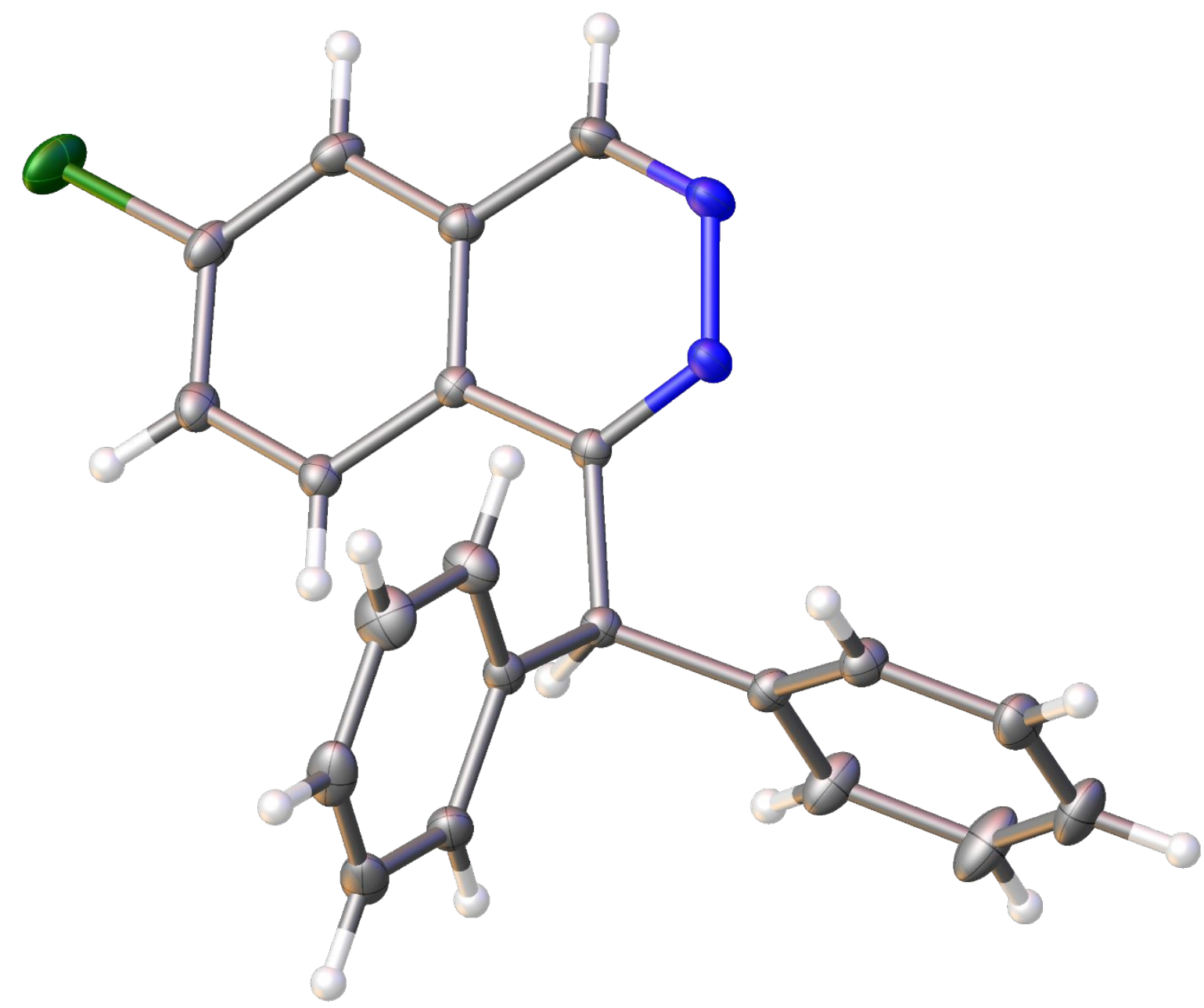




\begin{tabular}{|c|c|}
\hline \multicolumn{2}{|c|}{ Table S14 Crystal data and structure refinement for $\mathbf{2 j}$. } \\
\hline Identification code & mda835 \\
\hline Empirical formula & $\mathrm{C}_{21} \mathrm{H}_{15} \mathrm{ClN}_{2}$ \\
\hline Formula weight & 330.80 \\
\hline Temperature/K & $296(2)$ \\
\hline Crystal system & monoclinic \\
\hline Space group & $\mathrm{P} 2{ }_{1} / \mathrm{n}$ \\
\hline$a / \AA$ & $9.3645(4)$ \\
\hline $\mathrm{b} / \AA ̊ \AA$ & $18.4212(8)$ \\
\hline$c / \AA$ & $10.0791(5)$ \\
\hline$\alpha /^{\circ}$ & 90 \\
\hline$\beta /^{\circ}$ & $106.411(2)$ \\
\hline $\mathrm{V} /{ }^{\circ}$ & 90 \\
\hline Volume $/ \AA^{3}$ & $1667.86(13)$ \\
\hline$Z$ & 4 \\
\hline$\rho_{\text {calc }} \mathrm{g} / \mathrm{cm}^{3}$ & 1.317 \\
\hline$\mu / \mathrm{mm}^{-1}$ & 0.232 \\
\hline $\mathrm{F}(000)$ & 688.0 \\
\hline Crystal size $/ \mathrm{mm}^{3}$ & $0.290 \times 0.190 \times 0.170$ \\
\hline Radiation & $\operatorname{MoK} \alpha(\lambda=0.71073)$ \\
\hline $2 \Theta$ range for data collection ${ }^{\circ}$ & 4.422 to 63.274 \\
\hline Index ranges & $-13 \leq h \leq 13,-27 \leq k \leq 25,-14 \leq \mathrm{I} \leq 14$ \\
\hline Reflections collected & 51752 \\
\hline Independent reflections & $5591\left[R_{\text {int }}=0.0347, R_{\text {sigma }}=0.0182\right]$ \\
\hline Data/restraints/parameters & $5591 / 0 / 217$ \\
\hline Goodness-of-fit on $F^{2}$ & 1.069 \\
\hline Final R indexes $[\mid>=2 \sigma(I)]$ & $R_{1}=0.0393, w R_{2}=0.1007$ \\
\hline Final $\mathrm{R}$ indexes [all data] & $\mathrm{R}_{1}=0.0483, \mathrm{wR}_{2}=0.1080$ \\
\hline Largest diff. peak/hole / e $\AA^{-3}$ & $0.44 /-0.29$ \\
\hline
\end{tabular}




\begin{tabular}{|l|l|l|l|l|}
\hline \multicolumn{5}{|c|}{$\begin{array}{r}\text { Table S15 Fractional Atomic Coordinates }\left(\times 10^{4}\right) \text { and Equivalent Isotropic Displacement Parameters } \\
\left(\AA^{2} \times 10^{3}\right) \text { for 2j. } U_{\text {eq }} \text { is defined as } 1 / 3 \text { of of the trace of the orthogonalised } U_{\text {IJ }} \text { tensor. }\end{array}$} \\
\hline \multicolumn{1}{|c|}{ Atom } & \multicolumn{1}{|c|}{$\boldsymbol{y}$} & \multicolumn{1}{c|}{$\boldsymbol{z}$} & \multicolumn{1}{|c|}{ U(eq) } \\
\hline C1 & $6534.5(10)$ & $2760.4(5)$ & $1779.8(10)$ & $12.63(16)$ \\
\hline C2 & $7213.1(10)$ & $2396.6(5)$ & $746.6(10)$ & $14.07(17)$ \\
\hline C3 & $8224.3(12)$ & $1827.2(6)$ & $1100.2(11)$ & $20.8(2)$ \\
\hline C4 & $8842.9(14)$ & $1528.8(7)$ & $115.7(13)$ & $25.9(2)$ \\
\hline C5 & $8450.5(13)$ & $1798.9(7)$ & $-1223.2(12)$ & $24.3(2)$ \\
\hline C6 & $7443.5(13)$ & $2363.3(6)$ & $-1579.9(11)$ & $22.2(2)$ \\
\hline C7 & $6831.2(12)$ & $2661.9(6)$ & $-600.3(11)$ & $17.73(19)$ \\
\hline C8 & $7289.4(11)$ & $3483.4(5)$ & $2256.1(10)$ & $14.36(17)$ \\
\hline C9 & $8832.0(11)$ & $3543.0(6)$ & $2768.8(11)$ & $17.83(19)$ \\
\hline C10 & $9497.2(12)$ & $4209.5(6)$ & $3196.1(12)$ & $22.2(2)$ \\
\hline C11 & $8632.2(14)$ & $4828.5(7)$ & $3098.6(15)$ & $27.5(2)$ \\
\hline C12 & $7099.9(15)$ & $4779.5(7)$ & $2554.5(17)$ & $31.9(3)$ \\
\hline C13 & $6433.2(12)$ & $4108.8(6)$ & $2134.0(14)$ & $23.6(2)$ \\
\hline C14 & $6490.1(10)$ & $2251.3(5)$ & $2954.0(10)$ & $12.39(16)$ \\
\hline C15 & $5324.9(10)$ & $1722.2(5)$ & $2747.9(10)$ & $12.48(16)$ \\
\hline C16 & $4254.7(10)$ & $1595.0(5)$ & $1469.1(10)$ & $14.48(17)$ \\
\hline C17 & $3152.1(11)$ & $1088.7(6)$ & $1384.0(11)$ & $17.57(19)$ \\
\hline C18 & $3079.4(11)$ & $715.2(6)$ & $2576.9(12)$ & $18.9(2)$ \\
\hline C19 & $4105.5(11)$ & $817.1(6)$ & $3835.5(11)$ & $17.98(19)$ \\
\hline C20 & $5265.8(11)$ & $1317.9(5)$ & $3913.8(10)$ & $14.85(17)$ \\
\hline C21 & $6435.5(12)$ & $1434.3(6)$ & $5142.0(10)$ & $18.47(19)$ \\
\hline N1 & $7539.7(10)$ & $2318.2(5)$ & $4123.2(9)$ & $16.88(17)$ \\
\hline N2 & $7538.7(10)$ & $1886.1(5)$ & $5242.0(9)$ & $20.20(18)$ \\
\hline C11 & $1636.1(3)$ & $103.9(2)$ & $2437.5(4)$ & $31.01(9)$ \\
\hline & & & & \\
\hline
\end{tabular}




\begin{tabular}{|c|c|c|c|c|c|c|}
\hline \multicolumn{7}{|c|}{$\begin{array}{l}\text { Table S16 Anisotropic Displacement Parameters }\left(\AA^{2} \times 10^{3}\right) \text { for } \mathbf{2 j} \text {. The Anisotropic displacemen } \\
\text { factor exponent takes the form: }-2 \pi^{2}\left[h^{2} a^{* 2} U_{11}+2 h k a * b * U_{12}+\ldots\right] .\end{array}$} \\
\hline Atom & $\mathrm{U}_{11}$ & $U_{22}$ & $U_{33}$ & $U_{23}$ & $U_{13}$ & $U_{12}$ \\
\hline $\mathrm{C} 1$ & $11.6(4)$ & $13.8(4)$ & $13.0(4)$ & $0.3(3)$ & $4.1(3)$ & $-0.4(3)$ \\
\hline $\mathrm{C} 2$ & $13.5(4)$ & $15.8(4)$ & $13.8(4)$ & $-0.7(3)$ & $5.3(3)$ & $-2.5(3)$ \\
\hline $\mathrm{C3}$ & $21.3(5)$ & $23.7(5)$ & $18.7(5)$ & $-0.5(4)$ & $7.6(4)$ & $5.0(4)$ \\
\hline $\mathrm{C4}$ & $24.5(5)$ & $27.8(6)$ & $28.9(6)$ & $-4.0(4)$ & 13.1(4) & $5.7(4)$ \\
\hline C5 & 26.1(5) & $27.3(6)$ & $25.1(5)$ & $-8.5(4)$ & $16.4(4)$ & $-7.4(4)$ \\
\hline C6 & $28.4(5)$ & $25.4(5)$ & 16.0(4) & $-2.2(4)$ & $11.2(4)$ & $-10.3(4)$ \\
\hline $\mathrm{C7}$ & $20.4(4)$ & $18.2(5)$ & $15.3(4)$ & $0.5(3)$ & $6.1(3)$ & $-3.9(4)$ \\
\hline $\mathrm{C} 8$ & $14.5(4)$ & $13.7(4)$ & $16.3(4)$ & $0.4(3)$ & $6.8(3)$ & $-0.7(3)$ \\
\hline C9 & $14.9(4)$ & $17.7(5)$ & $20.7(5)$ & $1.1(4)$ & $4.7(3)$ & $-1.0(3)$ \\
\hline $\mathrm{C} 10$ & 19.2(5) & 23.1(5) & $24.3(5)$ & $-0.9(4)$ & $6.2(4)$ & $-6.8(4)$ \\
\hline $\mathrm{C} 11$ & $29.3(6)$ & $17.8(5)$ & $39.1(7)$ & $-5.0(5)$ & $15.5(5)$ & $-8.8(4)$ \\
\hline C12 & $26.9(6)$ & $15.2(5)$ & $57.9(9)$ & $-3.1(5)$ & $19.1(6)$ & $-0.4(4)$ \\
\hline $\mathrm{C} 13$ & 17.6(5) & 16.3(5) & $39.5(6)$ & $-0.4(4)$ & $12.4(4)$ & $0.3(4)$ \\
\hline C14 & $12.6(4)$ & 13.1(4) & $12.2(4)$ & $-0.8(3)$ & $4.8(3)$ & $-0.1(3)$ \\
\hline C15 & 11.4(4) & $12.3(4)$ & $14.3(4)$ & $-0.2(3)$ & $4.5(3)$ & $0.6(3)$ \\
\hline C16 & $12.4(4)$ & $14.6(4)$ & $15.8(4)$ & $-0.1(3)$ & $2.9(3)$ & $0.4(3)$ \\
\hline C17 & $13.0(4)$ & $16.9(5)$ & $20.7(5)$ & $-0.6(4)$ & $1.2(3)$ & $-0.6(3)$ \\
\hline $\mathrm{C} 18$ & 13.6(4) & 14.9(4) & $27.7(5)$ & $1.8(4)$ & $5.2(4)$ & $-2.4(3)$ \\
\hline C19 & 16.2(4) & 16.4(5) & $22.2(5)$ & $5.1(4)$ & $6.9(4)$ & $-0.8(3)$ \\
\hline $\mathrm{C} 20$ & 14.1(4) & $15.4(4)$ & $15.5(4)$ & $1.5(3)$ & $4.9(3)$ & $-0.1(3)$ \\
\hline $\mathrm{C} 21$ & 20.2(4) & 21.8(5) & 13.0(4) & $3.0(3)$ & $4.0(3)$ & $-2.3(4)$ \\
\hline N1 & $17.4(4)$ & $20.1(4)$ & $12.7(4)$ & $0.3(3)$ & $3.5(3)$ & $-4.0(3)$ \\
\hline N2 & $21.8(4)$ & $25.1(5)$ & $12.4(4)$ & $2.2(3)$ & $2.6(3)$ & $-5.4(3)$ \\
\hline $\mathrm{Cl} 1$ & $19.43(13)$ & $25.58(15)$ & $44.16(19)$ & $8.92(12)$ & $2.71(11)$ & $-9.94(10)$ \\
\hline
\end{tabular}




\begin{tabular}{|c|c|c|c|c|c|}
\hline \multicolumn{6}{|c|}{ Table S17 Bond Lengths for $\mathbf{2 j}$. } \\
\hline Atom & Atom & Length/Å & Atom & Atom & Length/Å \\
\hline $\mathrm{C} 1$ & C14 & $1.5198(13)$ & C12 & C13 & $1.3952(17)$ \\
\hline C1 & $\mathrm{C} 2$ & $1.5203(13)$ & C14 & N1 & $1.3097(12)$ \\
\hline C1 & $\mathrm{C} 8$ & $1.5207(14)$ & C14 & C15 & $1.4337(13)$ \\
\hline $\mathrm{C} 2$ & $\mathrm{C3}$ & $1.3904(15)$ & C15 & $\mathrm{C} 20$ & $1.4055(13)$ \\
\hline $\mathrm{C2}$ & $\mathrm{C7}$ & $1.3911(14)$ & C15 & C16 & $1.4109(13)$ \\
\hline $\mathrm{C3}$ & $\mathrm{C} 4$ & $1.3954(15)$ & $\mathrm{C} 16$ & C17 & $1.3759(14)$ \\
\hline $\mathrm{C4}$ & $\mathrm{C5}$ & $1.3869(18)$ & C17 & C18 & $1.4036(15)$ \\
\hline C5 & C6 & $1.3813(18)$ & C18 & C19 & $1.3708(15)$ \\
\hline C6 & C7 & $1.3878(15)$ & C18 & $\mathrm{Cl} 1$ & $1.7341(10)$ \\
\hline $\mathrm{C} 8$ & $\mathrm{C} 13$ & $1.3891(14)$ & C19 & $\mathrm{C} 20$ & $1.4105(14)$ \\
\hline $\mathrm{C} 8$ & C9 & $1.3943(14)$ & $\mathrm{C} 20$ & $\mathrm{C} 21$ & $1.4185(14)$ \\
\hline C9 & C10 & $1.3890(15)$ & C21 & N2 & $1.3081(14)$ \\
\hline $\mathrm{C} 10$ & C11 & $1.3859(17)$ & N1 & $\mathrm{N} 2$ & $1.3805(12)$ \\
\hline C11 & C12 & $1.3868(19)$ & & & \\
\hline
\end{tabular}

\begin{tabular}{|c|c|c|c|c|c|c|c|}
\hline \multicolumn{8}{|c|}{ Table S18 Bond Angles for $\mathbf{2 j}$. } \\
\hline Atom & Atom & Atom & Angle/ $/^{\circ}$ & Atom & Atom & Atom & Angle/ $/^{\circ}$ \\
\hline C14 & C1 & $\mathrm{C} 2$ & $111.72(8)$ & N1 & C14 & C15 & $122.77(9)$ \\
\hline C14 & C1 & $\mathrm{C} 8$ & $114.03(8)$ & N1 & C14 & $\mathrm{C} 1$ & $117.64(8)$ \\
\hline $\mathrm{C2}$ & $\mathrm{C} 1$ & $\mathrm{C} 8$ & $110.80(8)$ & C15 & C14 & $\mathrm{C} 1$ & $119.59(8)$ \\
\hline $\mathrm{C3}$ & $\mathrm{C} 2$ & $\mathrm{C7}$ & $118.94(9)$ & $\mathrm{C} 20$ & C15 & C16 & $119.35(9)$ \\
\hline $\mathrm{C3}$ & $\mathrm{C2}$ & $\mathrm{C} 1$ & $122.90(9)$ & $\mathrm{C} 20$ & C15 & C14 & $116.56(8)$ \\
\hline $\mathrm{C7}$ & $\mathrm{C} 2$ & C1 & $118.14(9)$ & C16 & C15 & C14 & $124.08(9)$ \\
\hline $\mathrm{C2}$ & $\mathrm{C} 3$ & C4 & $120.29(10)$ & C17 & C16 & C15 & $119.76(9)$ \\
\hline $\mathrm{C5}$ & $\mathrm{C4}$ & $\mathrm{C} 3$ & $120.09(11)$ & C16 & C17 & C18 & $119.74(9)$ \\
\hline $\mathrm{C} 6$ & $\mathrm{C} 5$ & $\mathrm{C} 4$ & $119.81(10)$ & C19 & $\mathrm{C} 18$ & C17 & $122.32(9)$ \\
\hline $\mathrm{C} 5$ & $\mathrm{C} 6$ & $\mathrm{C} 7$ & $120.13(10)$ & C19 & $\mathrm{C} 18$ & $\mathrm{Cl} 1$ & $119.37(8)$ \\
\hline $\mathrm{C} 6$ & $\mathrm{C7}$ & $\mathrm{C} 2$ & $120.74(10)$ & C17 & $\mathrm{C} 18$ & $\mathrm{Cl} 1$ & 118.31(8) \\
\hline $\mathrm{C} 13$ & $\mathrm{C} 8$ & C9 & 118.63(9) & $\mathrm{C} 18$ & C19 & $\mathrm{C} 20$ & 117.96(9) \\
\hline $\mathrm{C} 13$ & $\mathrm{C} 8$ & $\mathrm{C} 1$ & 119.55(9) & $\mathrm{C} 15$ & $\mathrm{C} 20$ & C19 & $120.76(9)$ \\
\hline C9 & $\mathrm{C} 8$ & $\mathrm{C} 1$ & 121.78(9) & C15 & $\mathrm{C} 20$ & $\mathrm{C} 21$ & $116.51(9)$ \\
\hline C10 & C9 & $\mathrm{C} 8$ & $120.76(10)$ & C19 & $\mathrm{C} 20$ & $\mathrm{C} 21$ & 122.71(9) \\
\hline C11 & C10 & C9 & $120.22(10)$ & $\mathrm{N} 2$ & $\mathrm{C} 21$ & $\mathrm{C} 20$ & $124.18(9)$ \\
\hline C10 & C11 & C12 & $119.56(11)$ & C14 & N1 & N2 & $120.47(9)$ \\
\hline $\mathrm{C} 11$ & $\mathrm{C} 12$ & $\mathrm{C} 13$ & $120.13(11)$ & $\mathrm{C} 21$ & $\mathrm{~N} 2$ & N1 & 119.12(9) \\
\hline $\mathrm{C} 8$ & C13 & C12 & $120.66(10)$ & & & & \\
\hline
\end{tabular}




\begin{tabular}{|l|l|l|l|l|}
\hline \multicolumn{5}{|c|}{ Table S19 Hydrogen Atom Coordinates $\left(\AA \times 10^{4}\right)$ and Isotropic Displacement Parameters $\left(\AA^{2} \times 10^{3}\right)$ for } \\
\hline \multicolumn{1}{|c|}{ Atom } & \multicolumn{1}{|c|}{$\boldsymbol{y}$} & \multicolumn{1}{c|}{$\boldsymbol{z}$} & U(eq) \\
\hline H1 & 5497.65 & 2870.41 & 1281.62 & 15 \\
\hline H3 & 8489.57 & 1644.46 & 1996.81 & 25 \\
\hline H4 & 9519.23 & 1148.16 & 357.72 & 31 \\
\hline H5 & 8864.11 & 1600.65 & -1878.86 & 29 \\
\hline H6 & 7175.34 & 2543.67 & -2478.29 & 27 \\
\hline H7 & 6157.97 & 3043.48 & -847.04 & 21 \\
\hline H9 & 9422.37 & 3131.77 & 2825.44 & 21 \\
\hline H10 & 10526.38 & 4240.56 & 3548.7 & 27 \\
\hline H11 & 9076.38 & 5273.62 & 3395.99 & 33 \\
\hline H12 & 6515.65 & 5194.58 & 2469.78 & 38 \\
\hline H13 & 5405.16 & 4080.08 & 1768.25 & 28 \\
\hline H16 & 4294.3 & 1852.47 & 686.63 & 17 \\
\hline H17 & 2457.86 & 994.36 & 539.31 & 21 \\
\hline H19 & 4038.62 & 563.83 & 4613.92 & 22 \\
\hline H21 & 6416.81 & 1172.85 & 5926.23 & 22 \\
\hline
\end{tabular}




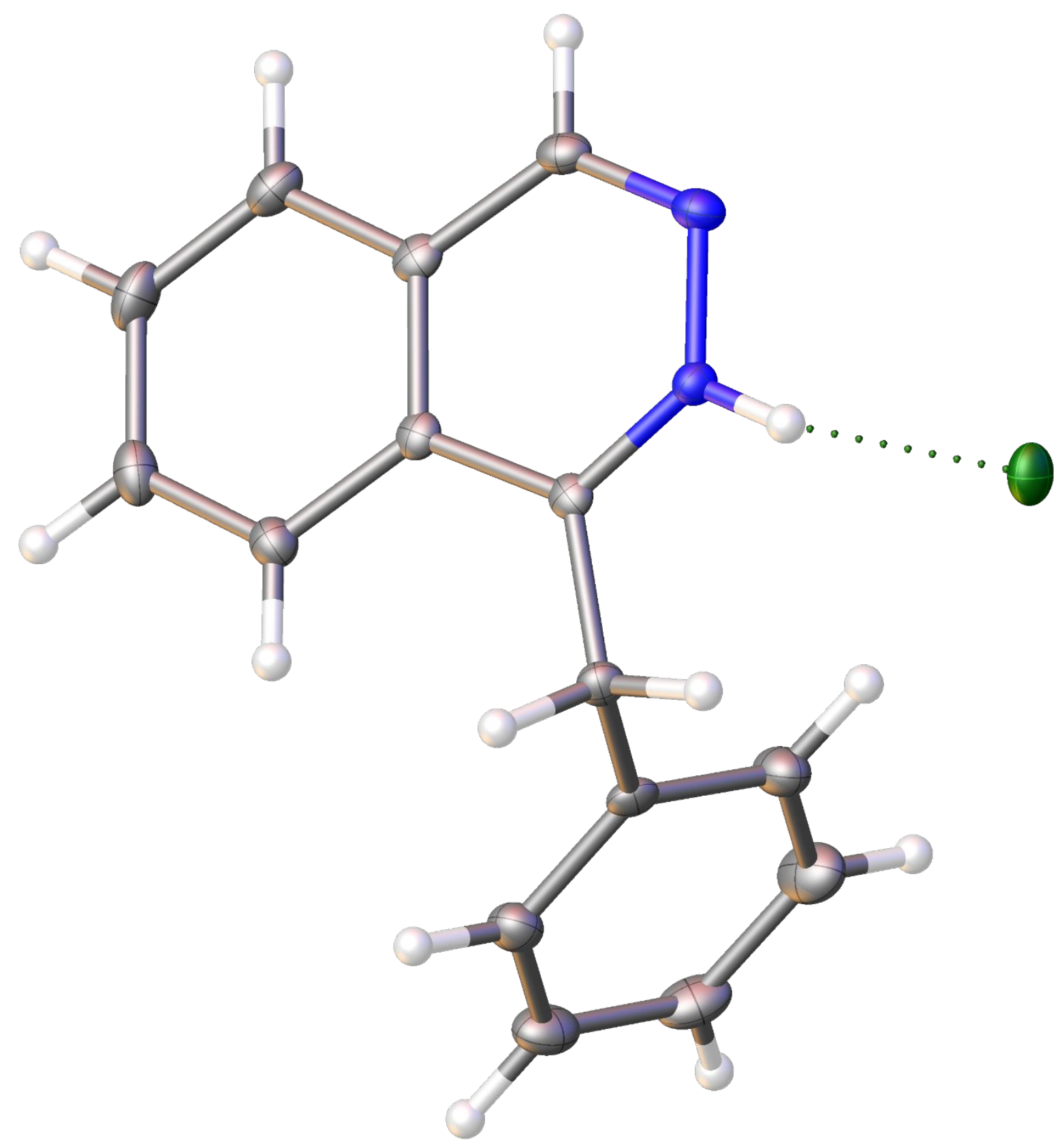




\begin{tabular}{|c|c|}
\hline \multicolumn{2}{|c|}{ Table S20 Crystal data and structure refinement for $3 a . \mathbf{H C l}$. } \\
\hline Identification code & mda527 \\
\hline Empirical formula & $\mathrm{C}_{15} \mathrm{H}_{13} \mathrm{CIN}_{2}$ \\
\hline Formula weight & 256.72 \\
\hline Temperature/K & $100(2)$ \\
\hline Crystal system & monoclinic \\
\hline Space group & $\mathrm{P} 2{ }_{1} / \mathrm{c}$ \\
\hline$a / \AA$ & $12.7095(5)$ \\
\hline $\mathrm{b} / \AA ̊ \AA$ & $11.4141(4)$ \\
\hline$c / \AA ̊$ & $8.9423(3)$ \\
\hline$\alpha /^{\circ}$ & 90 \\
\hline$\beta /^{\circ}$ & $100.5190(10)$ \\
\hline $\mathrm{Y} /{ }^{\circ}$ & 90 \\
\hline Volume $/ \AA^{3}$ & $1275.44(8)$ \\
\hline Z & 4 \\
\hline$\rho_{\text {calc }} \mathrm{g} / \mathrm{cm}^{3}$ & 1.337 \\
\hline$\mu / \mathrm{mm}^{-1}$ & 2.448 \\
\hline $\mathrm{F}(000)$ & 536.0 \\
\hline Crystal size $/ \mathrm{mm}^{3}$ & $0.190 \times 0.180 \times 0.050$ \\
\hline Radiation & $\operatorname{CuKa}(\lambda=1.54178)$ \\
\hline $2 \Theta$ range for data collection $/^{\circ}$ & 7.074 to 130.34 \\
\hline Index ranges & $-14 \leq h \leq 14,-13 \leq k \leq 13,-10 \leq \mathrm{I} \leq 10$ \\
\hline Reflections collected & 20684 \\
\hline Independent reflections & $2173\left[R_{\text {int }}=0.0277, R_{\text {sigma }}=0.0142\right]$ \\
\hline Data/restraints/parameters & $2173 / 0 / 163$ \\
\hline Goodness-of-fit on $F^{2}$ & 1.056 \\
\hline Final $R$ indexes $[I>=2 \sigma(I)]$ & $R_{1}=0.0273, w R_{2}=0.0726$ \\
\hline Final $\mathrm{R}$ indexes [all data] & $\mathrm{R}_{1}=0.0278, \mathrm{wR}_{2}=0.0729$ \\
\hline Largest diff. peak/hole / e $\AA^{-3}$ & $0.25 /-0.32$ \\
\hline
\end{tabular}




\begin{tabular}{|l|l|l|l|l|}
\hline \multicolumn{5}{|c|}{$\begin{array}{c}\text { Table S21 Fractional Atomic Coordinates }\left(\times 10^{4}\right) \text { and Equivalent Isotropic Displacement Parameters } \\
\left(\AA^{2} \times 10^{3}\right)\end{array}$ for 3a.HCl. $U_{\text {eq }}$ is defined as $1 / 3$ of of the trace of the orthogonalised $U_{\text {IJ }}$ tensor. } \\
\hline \multicolumn{1}{|c|}{ Atom } & \multicolumn{1}{|c|}{$\boldsymbol{x}$} & \multicolumn{1}{|c|}{$\boldsymbol{y}$} & \multicolumn{1}{c|}{$\boldsymbol{z}$} & \multicolumn{1}{c|}{ U(eq) } \\
\hline C1 & $4328.0(10)$ & $7001.9(12)$ & $5738.6(15)$ & $20.8(3)$ \\
\hline C2 & $5366.7(11)$ & $6765.4(14)$ & $5550.1(16)$ & $26.6(3)$ \\
\hline C3 & $5996.6(10)$ & $6003.1(13)$ & $6528.0(16)$ & $24.4(3)$ \\
\hline C4 & $5594.8(11)$ & $5474.6(12)$ & $7696.8(17)$ & $23.3(3)$ \\
\hline C5 & $4550.4(10)$ & $5704.5(12)$ & $7879.7(15)$ & $18.7(3)$ \\
\hline C6 & $3909.7(9)$ & $6465.9(11)$ & $6900.7(13)$ & $13.8(3)$ \\
\hline C7 & $2764.6(9)$ & $6725.0(11)$ & $7083.0(13)$ & $13.5(3)$ \\
\hline C8 & $1974.8(9)$ & $6275.7(11)$ & $5751.0(13)$ & $11.7(3)$ \\
\hline C9 & $1773.6(9)$ & $5061.3(11)$ & $5435.2(14)$ & $12.6(3)$ \\
\hline C10 & $1072.6(9)$ & $4787.1(11)$ & $4065.9(14)$ & $13.4(3)$ \\
\hline C11 & $855.2(10)$ & $3611.9(12)$ & $3651.0(15)$ & $18.0(3)$ \\
\hline C12 & $1330.6(11)$ & $2738.1(12)$ & $4593.3(16)$ & $20.9(3)$ \\
\hline C13 & $2019.8(10)$ & $3007.3(12)$ & $5966.3(15)$ & $19.2(3)$ \\
\hline C14 & $2238.9(10)$ & $4150.1(11)$ & $6397.5(14)$ & $15.6(3)$ \\
\hline C15 & $598.4(9)$ & $5728.8(12)$ & $3139.4(14)$ & $15.0(3)$ \\
\hline N1 & $1464.7(8)$ & $7050.7(9)$ & $4800.0(11)$ & $12.6(2)$ \\
\hline N2 & $762.8(8)$ & $6830.7(10)$ & $3479.9(12)$ & $15.7(2)$ \\
\hline Cl & $1756.3(2)$ & $9643.1(2)$ & $5132.8(3)$ & $18.24(12)$ \\
\hline
\end{tabular}

\begin{tabular}{|c|c|c|c|c|c|c|}
\hline Atom & $\mathrm{U}_{11}$ & $U_{22}$ & $\mathbf{U}_{33}$ & $U_{23}$ & $U_{13}$ & $\mathrm{U}_{12}$ \\
\hline C1 & $16.7(6)$ & $26.2(7)$ & $18.7(7)$ & $3.3(5)$ & $1.1(5)$ & $-1.4(5)$ \\
\hline $\mathrm{C} 2$ & $18.7(7)$ & 38.1(9) & $24.5(7)$ & $1.9(6)$ & $7.9(6)$ & $-4.9(6)$ \\
\hline $\mathrm{C3}$ & $12.1(6)$ & $31.2(8)$ & $30.0(7)$ & $-8.8(6)$ & $3.8(5)$ & $-0.5(6)$ \\
\hline $\mathrm{C4}$ & 16.9(7) & $23.8(7)$ & $26.8(7)$ & $-1.8(6)$ & $-2.3(6)$ & $4.1(5)$ \\
\hline C5 & $18.2(6)$ & $21.3(7)$ & $15.8(6)$ & $0.5(5)$ & $1.2(5)$ & $-0.3(5)$ \\
\hline $\mathrm{C} 6$ & $12.6(6)$ & $14.7(6)$ & 13.1(6) & $-4.7(5)$ & $0.0(5)$ & $-2.2(5)$ \\
\hline $\mathrm{C7}$ & 14.1(6) & $14.0(6)$ & $12.3(6)$ & $-1.7(5)$ & $2.2(5)$ & $-0.3(5)$ \\
\hline $\mathrm{C} 8$ & $9.0(5)$ & $14.4(6)$ & $12.8(6)$ & $0.8(5)$ & $5.3(5)$ & $0.7(5)$ \\
\hline C9 & $10.3(6)$ & 14.1(6) & $14.6(6)$ & $-0.3(5)$ & $6.0(5)$ & $-0.5(5)$ \\
\hline C10 & $9.8(6)$ & $16.9(6)$ & 15.0(6) & $-1.7(5)$ & $6.0(5)$ & $-1.1(5)$ \\
\hline C11 & $16.4(6)$ & $18.5(7)$ & $20.1(6)$ & $-5.5(5)$ & $5.8(5)$ & $-4.5(5)$ \\
\hline $\mathrm{C} 12$ & $24.1(7)$ & $13.5(6)$ & $27.8(7)$ & $-4.3(5)$ & $11.8(6)$ & $-4.2(5)$ \\
\hline $\mathrm{C} 13$ & $20.6(7)$ & 15.1(6) & $24.1(7)$ & $4.8(5)$ & $10.2(5)$ & $2.4(5)$ \\
\hline C14 & $14.2(6)$ & $16.7(6)$ & $16.6(6)$ & $2.0(5)$ & $4.2(5)$ & $1.1(5)$ \\
\hline C15 & $10.4(6)$ & $20.6(7)$ & $13.8(6)$ & $-1.4(5)$ & $2.0(5)$ & $-0.9(5)$ \\
\hline N1 & $11.9(5)$ & $11.8(5)$ & $14.1(5)$ & $-0.8(4)$ & $1.9(4)$ & $-0.1(4)$ \\
\hline N2 & $13.0(5)$ & $19.3(6)$ & $14.0(5)$ & $0.7(4)$ & $0.2(4)$ & $1.1(4)$ \\
\hline $\mathrm{Cl}$ & 23.65(19) & $11.66(17)$ & 20.66(19) & $-0.44(11)$ & $7.33(13)$ & $0.00(11)$ \\
\hline
\end{tabular}




\begin{tabular}{|c|c|c|c|c|c|}
\hline \multicolumn{6}{|c|}{ Table S23 Bond Lengths for 3a.HCl. } \\
\hline Atom & Atom & Length/Å & Atom & Atom & Length/Å \\
\hline C1 & C2 & $1.3875(19)$ & C9 & C14 & $1.4093(18)$ \\
\hline $\mathrm{C} 1$ & C6 & $1.3922(18)$ & C9 & C10 & $1.4117(17)$ \\
\hline $\mathrm{C} 2$ & C3 & $1.381(2)$ & C10 & C11 & $1.4056(18)$ \\
\hline $\mathrm{C3}$ & C4 & $1.383(2)$ & C10 & C15 & $1.4222(18)$ \\
\hline C4 & C5 & $1.3918(19)$ & C11 & C12 & $1.373(2)$ \\
\hline C5 & C6 & $1.3872(18)$ & C12 & C13 & $1.406(2)$ \\
\hline C6 & $\mathrm{C7}$ & $1.5228(16)$ & C13 & C14 & $1.3742(19)$ \\
\hline $\mathrm{C7}$ & $\mathrm{C} 8$ & $1.5009(16)$ & C15 & $\mathrm{N} 2$ & $1.3020(17)$ \\
\hline C8 & N1 & $1.3132(16)$ & N1 & N2 & $1.3667(14)$ \\
\hline $\mathrm{C} 8$ & C9 & $1.4283(17)$ & & & \\
\hline
\end{tabular}

\begin{tabular}{|c|c|c|c|c|c|c|c|}
\hline \multicolumn{8}{|c|}{ Table S24 Bond Angles for 3a.HCl. } \\
\hline Atom & Atom & Atom & Angle/ $/^{\circ}$ & Atom & Atom & Atom & Angle $^{\circ}$ \\
\hline C2 & C1 & C6 & 120.44(13) & C14 & C9 & C8 & 123.74(11) \\
\hline C3 & C2 & C1 & $120.00(13)$ & C10 & C9 & $\mathrm{C} 8$ & $116.65(11)$ \\
\hline $\mathrm{C} 2$ & C3 & C4 & 120.14(13) & C11 & C10 & C9 & $120.20(12)$ \\
\hline $\mathrm{C3}$ & $\mathrm{C} 4$ & $\mathrm{C} 5$ & 119.89(13) & C11 & C10 & C15 & $121.70(12)$ \\
\hline C6 & C5 & C4 & $120.43(13)$ & C9 & C10 & C15 & $118.10(11)$ \\
\hline $\mathrm{C5}$ & C6 & C1 & $119.09(12)$ & C12 & C11 & C10 & $119.22(12)$ \\
\hline C5 & C6 & C7 & 121.09(11) & C11 & $\mathrm{C} 12$ & C13 & $120.77(12)$ \\
\hline $\mathrm{C} 1$ & $\mathrm{C6}$ & $\mathrm{C7}$ & 119.81(11) & C14 & $\mathrm{C} 13$ & $\mathrm{C} 12$ & $120.97(12)$ \\
\hline $\mathrm{C} 8$ & $\mathrm{C7}$ & C6 & $111.25(10)$ & $\mathrm{C} 13$ & C14 & C9 & $119.22(12)$ \\
\hline N1 & $\mathrm{C} 8$ & C9 & $118.44(11)$ & $\mathrm{N} 2$ & C15 & C10 & $124.13(11)$ \\
\hline N1 & $\mathrm{C} 8$ & $\mathrm{C7}$ & $117.60(11)$ & $\mathrm{C} 8$ & N1 & $\mathrm{N} 2$ & $127.07(11)$ \\
\hline C9 & $\mathrm{C} 8$ & $\mathrm{C} 7$ & $123.93(11)$ & $\mathrm{C} 15$ & $\mathrm{~N} 2$ & N1 & $115.53(10)$ \\
\hline C14 & C9 & C10 & 119.61(12) & & & & \\
\hline
\end{tabular}

\begin{tabular}{|c|c|c|c|c|}
\hline \multicolumn{5}{|c|}{$\begin{array}{c}\text { Table S25 Hydrogen Atom Coordinates }\left(\AA \times 10^{4}\right) \text { and Isotropic Displacement Parameters }\left(\AA^{2} \times 10^{3}\right) f \\
\text { 3a. } \mathbf{H C l} .\end{array}$} \\
\hline Atom & $x$ & $y$ & $z$ & $U(e q)$ \\
\hline $\mathrm{H} 1$ & 3899.36 & 7533 & 5070.39 & 25 \\
\hline $\mathrm{H} 2$ & 5644.49 & 7127.98 & 4748.22 & 32 \\
\hline $\mathrm{H} 3$ & 6707.47 & 5841.47 & 6397.4 & 29 \\
\hline $\mathrm{H} 4$ & 6030.96 & 4955.35 & 8373.79 & 28 \\
\hline $\mathrm{H} 5$ & 4274.38 & 5337.7 & 8680.24 & 22 \\
\hline $\mathrm{H} 7 \mathrm{~A}$ & 2624.7 & 6352.52 & 8027.18 & 16 \\
\hline H7B & 2671.78 & 7581.43 & 7176.78 & 16 \\
\hline H11 & 384.89 & 3424.64 & 2729.31 & 22 \\
\hline $\mathrm{H} 12$ & 1192.26 & 1942.11 & 4314.52 & 25 \\
\hline $\mathrm{H} 13$ & 2338.82 & 2390.2 & 6605.35 & 23 \\
\hline $\mathrm{H} 14$ & 2698.63 & 4323.87 & 7333.33 & 19 \\
\hline $\mathrm{H} 15$ & 132.25 & 5541.97 & 2213.76 & 18 \\
\hline $\mathrm{H} 100$ & 1588.27 & 7792.22 & 5038.7 & 15 \\
\hline
\end{tabular}


VIII- Mechanistic proposal

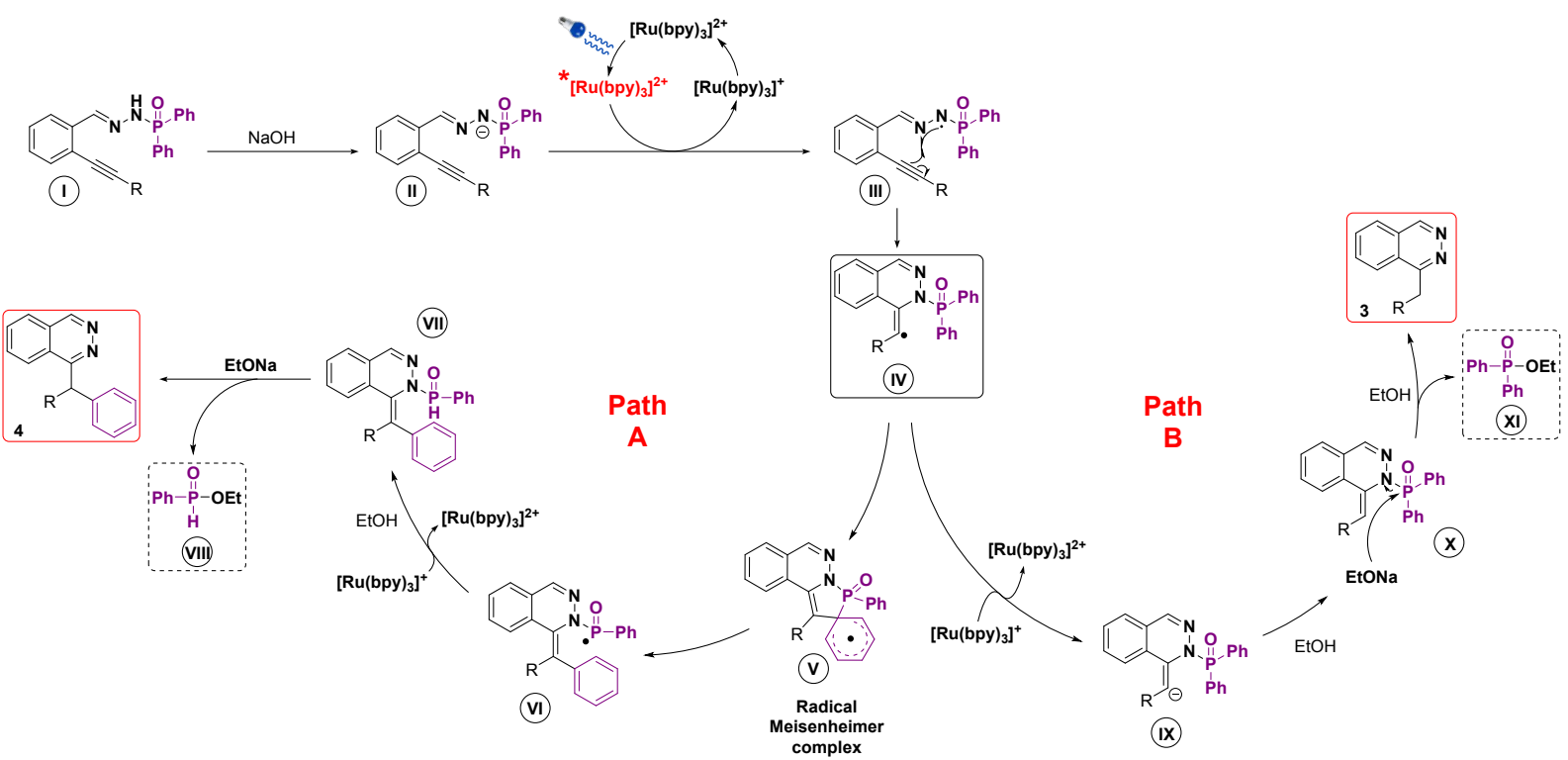

Scheme S1: Mechanistic proposal 


\section{IX- Phosphorus by-products}

The by-product VIII, formed after the "phospho-Smiles" rearrangement was detected using LC-MS.
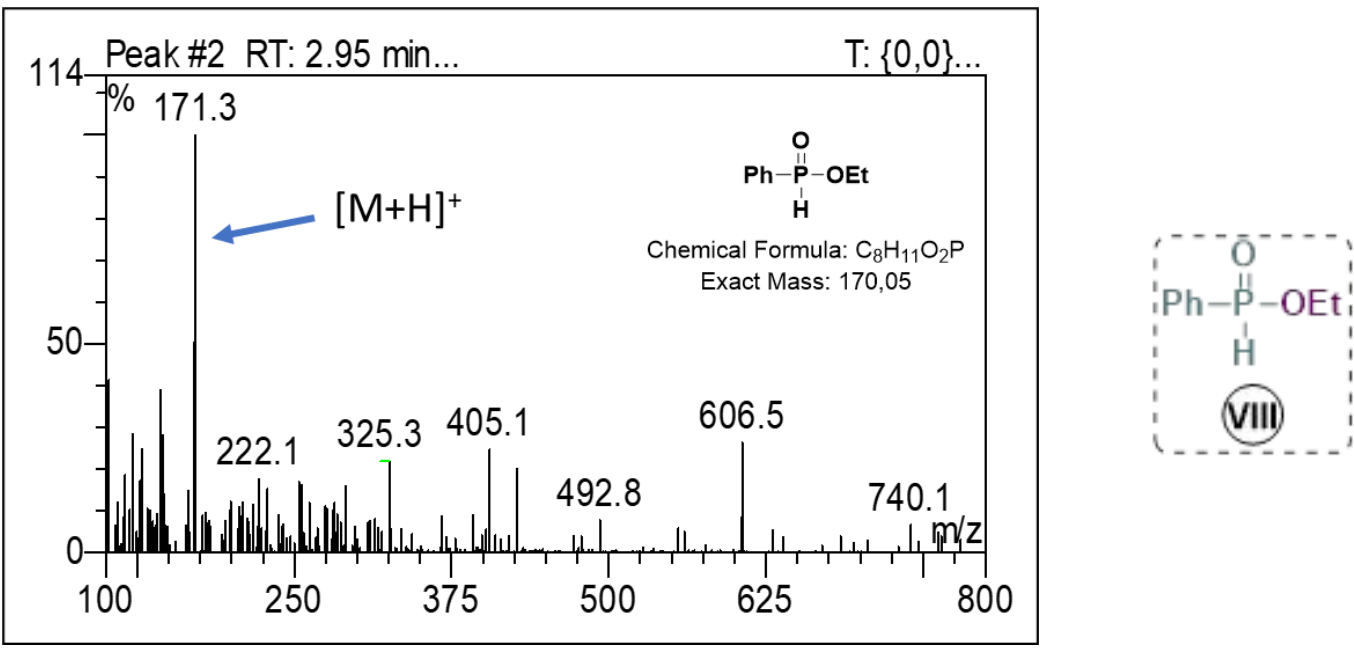

The by-product XI, also named 5 in the manuscript, which is formed when no "phospho-Smiles" rearrangement occurs was isolated and characterized. Its spectral data were consistent with the literature. ${ }^{[3]}$

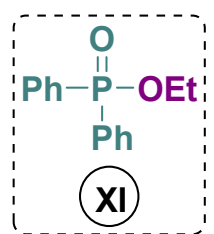

${ }^{1} \mathrm{H}$ NMR $(300 \mathrm{MHz}$, Chloroform-d) $\delta 7.80$ (dd, $J=11.4,7.5 \mathrm{~Hz}, 4 \mathrm{H}$ ), 7.48 (dd, $J=14.3,7.2 \mathrm{~Hz}, 6 \mathrm{H}$ ), 4.19 $-3.96(\mathrm{~m}, 2 \mathrm{H}), 1.35(\mathrm{t}, J=6.8 \mathrm{~Hz}, 3 \mathrm{H})$.

${ }^{31} \mathrm{P}$ NMR (121 MHz, Chloroform-d) $\delta 31.44$.

${ }^{13} \mathrm{C}\left\{{ }^{1} \mathrm{H}\right\}$ NMR (75 MHz, Chloroform- $d$ ) $\delta 132.1$ (d, $\left.J=1.8 \mathrm{~Hz}\right), 131.7(\mathrm{~d}, J=136.8 \mathrm{~Hz}), 131.7(\mathrm{~d}, J=10.0$ $\mathrm{Hz}), 128.6(\mathrm{~d}, J=13.0 \mathrm{~Hz}), 61.2(\mathrm{~d}, J=5.8 \mathrm{~Hz}), 16.5(\mathrm{~d}, J=6.4 \mathrm{~Hz})$. 

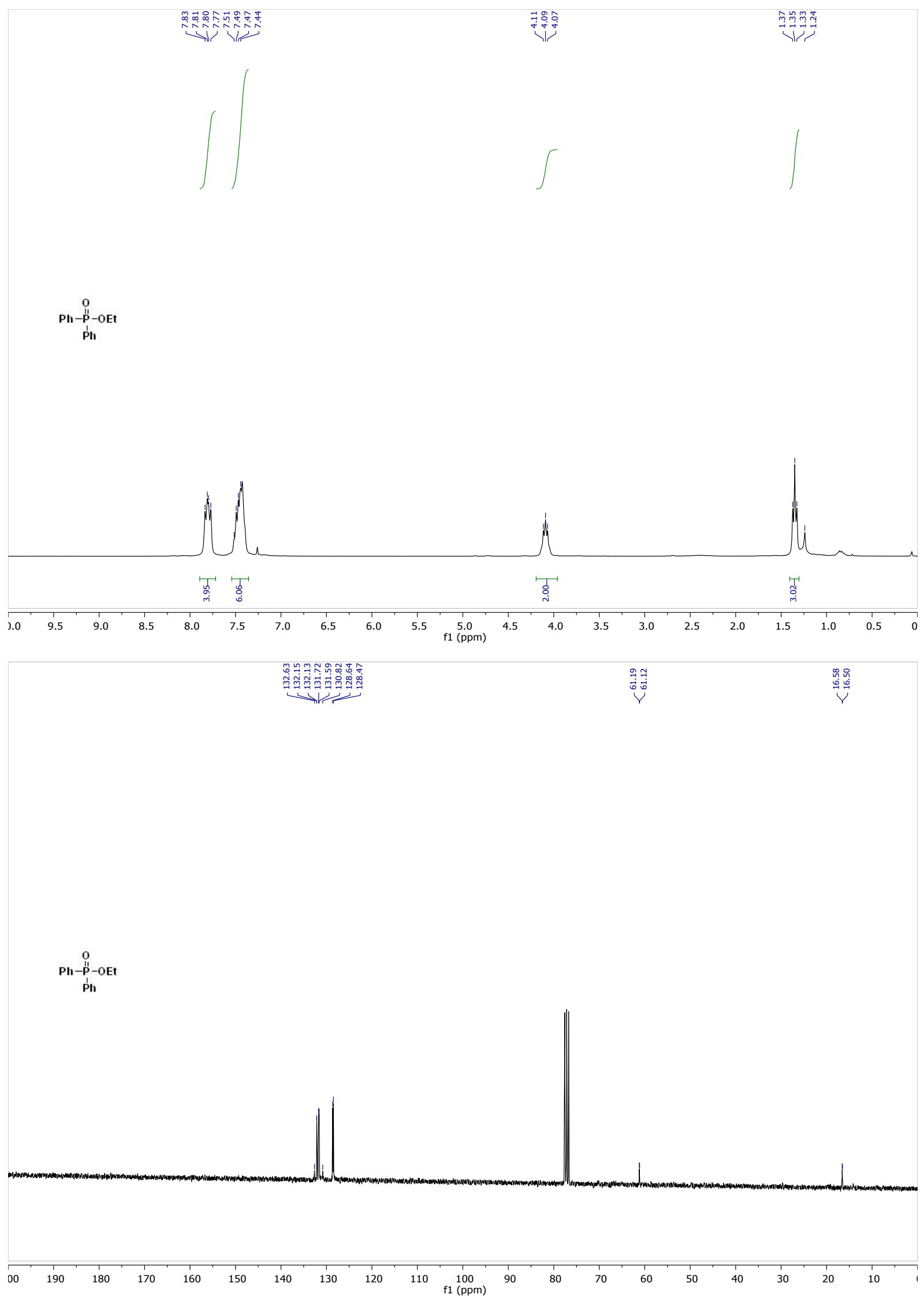

S110 


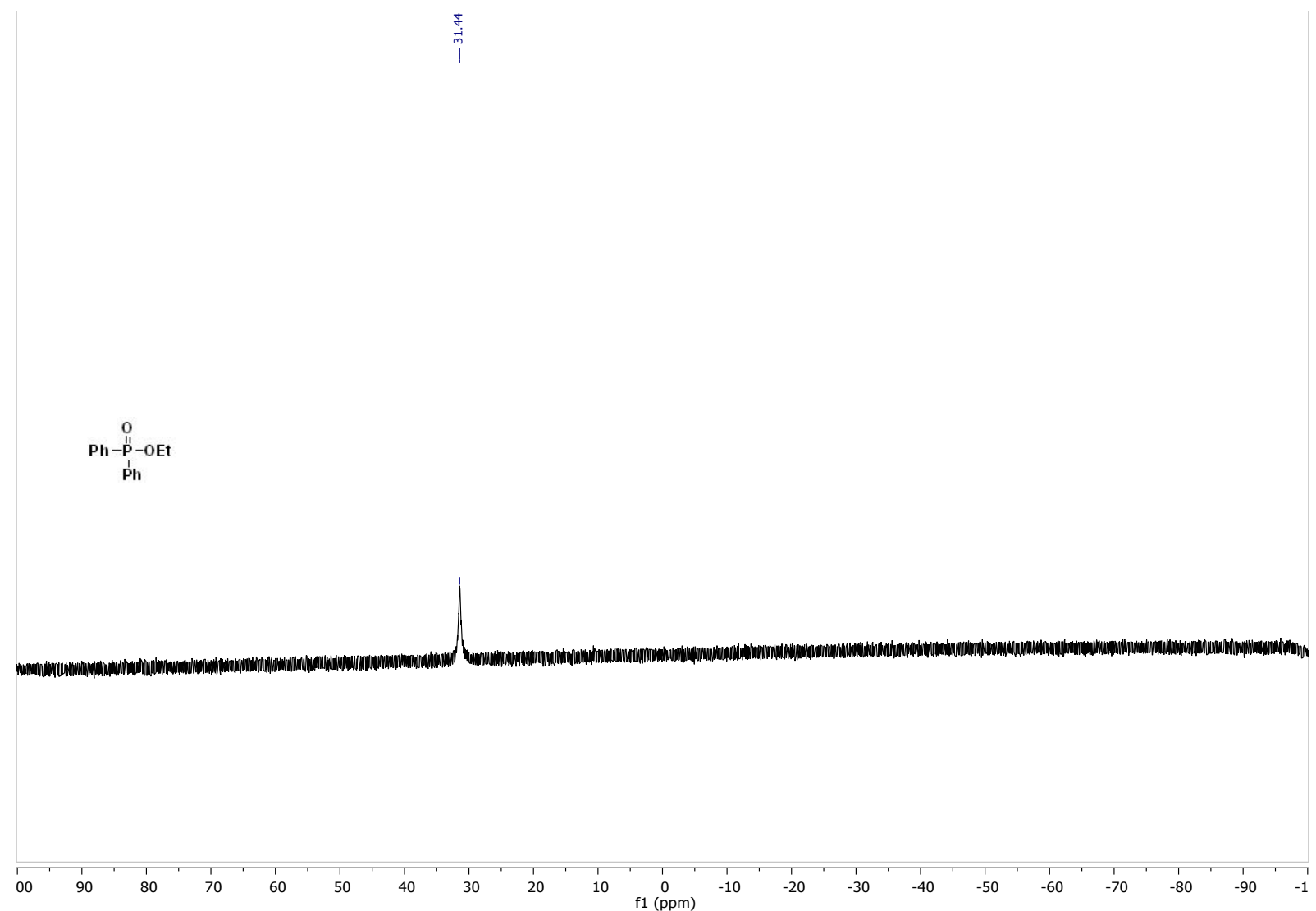




\section{X- References}

[1] De Abreu, M.; Selkti, M.; Belmont, P.; Brachet, E. Phospho-ramidates as Transient Precursors of Nitrogen-Centered Radical Under Visible-Light Irradiation: Application to the Synthesis of Phthalazine Derivatives. Adv. Synth. Catal. 2020, 362 (11), 2216-2222.

[2] Brachet, E.; Marzo, L.; Selkti, M.; König, B.; Belmont, P. Visible light amination/Smiles cascade: access to phthalazine derivatives. Chem. Sci. 2016, 7, 5002-5006.

[3] Hu, G.; Chen, W.; Fu, T.; Peng, Z.; Qiao, H.; Gao, Y.; Zhao, Y. Nickel-Catalyzed C-P Cross-Coupling of Arylboronic Acids with P(O)H Compounds. Org. Lett. 2013, 15, 5362-5365. 